\title{
As-Run Physics Analysis for the UCSB-1 Experiment in the Advanced Test Reactor
}

\author{
J. W. Nielsen
}

September 2015

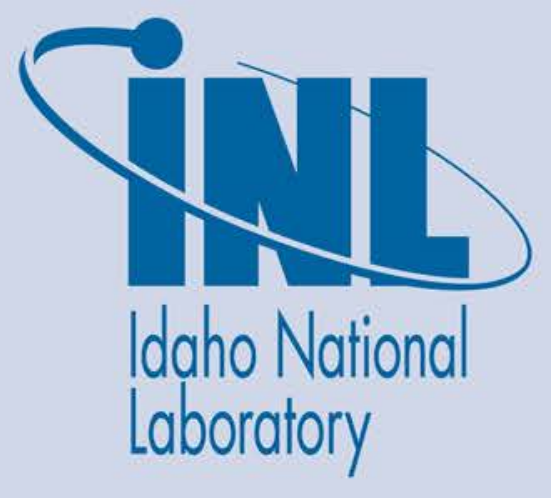

The INL is a U.S. Department of Energy National Laboratory operated by Battelle Energy Alliance 


\section{DISCLAIMER}

This information was prepared as an account of work sponsored by an agency of the U.S. Government. Neither the U.S. Government nor any agency thereof, nor any of their employees, makes any warranty, expressed or implied, or assumes any legal liability or responsibility for the accuracy, completeness, or usefulness, of any information, apparatus, product, or process disclosed, or represents that its use would not infringe privately owned rights. References herein to any specific commercial product, process, or service by trade name, trade mark, manufacturer, or otherwise, does not necessarily constitute or imply its endorsement, recommendation, or favoring by the U.S. Government or any agency thereof. The views and opinions of authors expressed herein do not necessarily state or reflect those of the U.S. Government or any agency thereof. 
INL/EXT-15-34225

Revision 0

\title{
As-Run Physics Analysis for the UCSB-1 Experiment in the Advanced Test Reactor
}

\author{
J. W. Nielsen
}

September 2015

Idaho National Laboratory

Experiment Design and Analysis

Idaho Falls, Idaho 83415

http://www.inl.gov

Prepared for the

U.S. Department of Energy Office of

Under DOE Idaho Operations Office

Contract DE-AC07-05ID14517 



\section{Experiment Design and Analysis}

\section{As-Run Physics Analysis for the UCSB-1 Experiment in the Advanced Test Reactor}

INL/EXT-15-34225

September 2015

Approved by:

J. W. Nielsen

Date

Nuclear Engineer

B. J. Gross

Nuclear Engineer

Date

T. L. Maddock

Date

Experiment Manager

D. J. Schoonen

Date

Experiment Design and Analysis Manager 


\section{SUMMARY}

The University of California Santa Barbara (UCSB) -1 experiment was irradiated in the A-10 position of the ATR. The experiment was irradiated during cycles 145A, 145B, 146A, and 146B. Capsule 6A was removed from the test train following Cycle 145A and replaced with Capsule 6B. This report documents the as-run physics analysis in support of Post-Irradiation Examination (PIE) of the test. This report documents the as-run fluence and displacements per atom (DPA) for each capsule of the experiment based on as-run operating history of the ATR. Average as-run heating rates for each capsule are also presented in this report to support the thermal analysis. 


\section{CONTENTS}

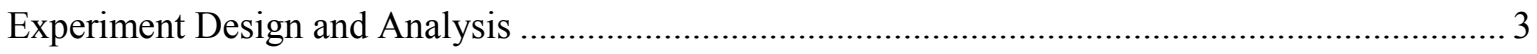

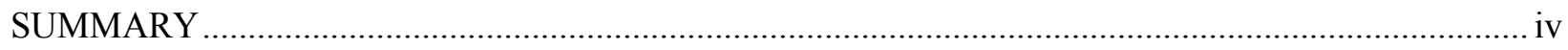

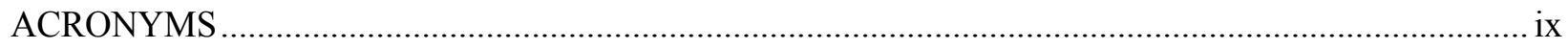

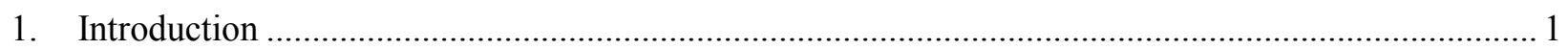

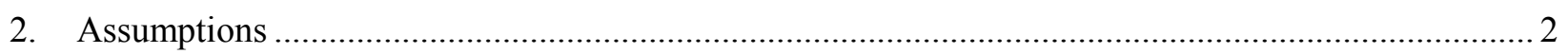

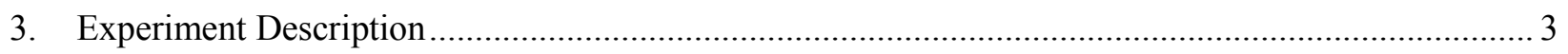

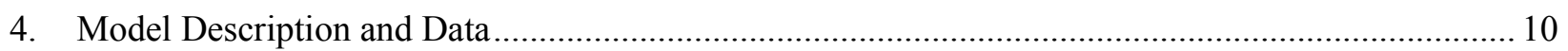

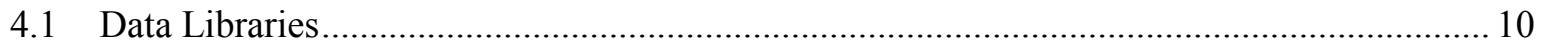

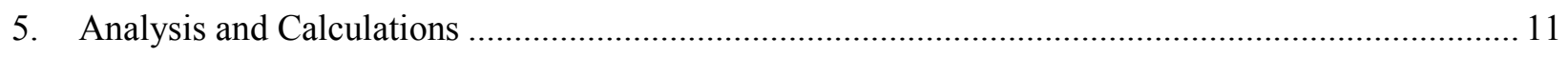

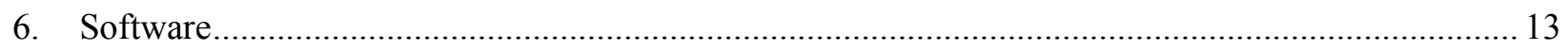

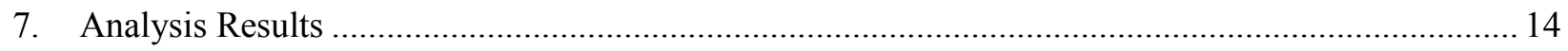

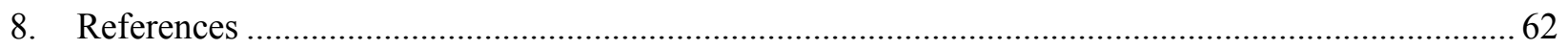

\section{FIGURES}

Figure 1. Radial cross section view of the ATR core, A-10 irradiation test position. ................................ 4

Figure 2. Capsule assembly schematic (lengths are in inches) [1] ...................................................... 8

\section{TABLES}

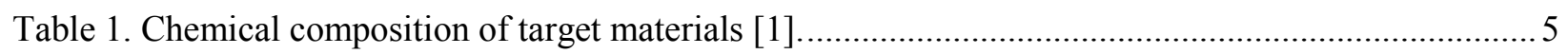

Table 2. Target temperature and dose for each UCSB capsule [1] ...................................................... 7

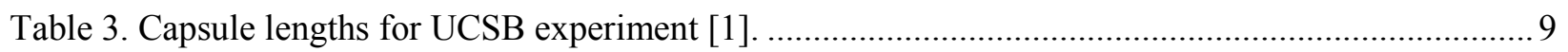

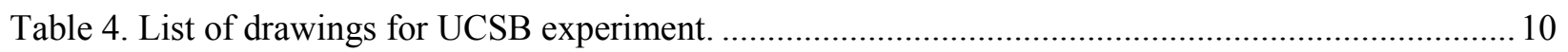

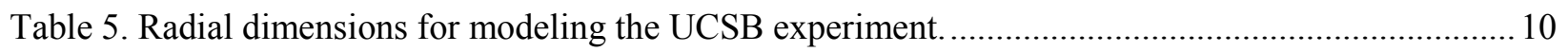

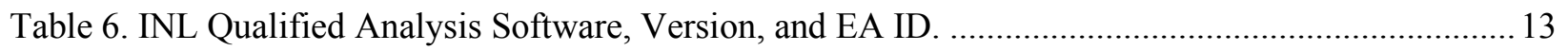

Table 7: Computer Configurations for INL Qualified MCNP5 installations........................................... 13

Table 8. Calculated as run heating rates for UCSB packets for Cycle 145A........................................... 15

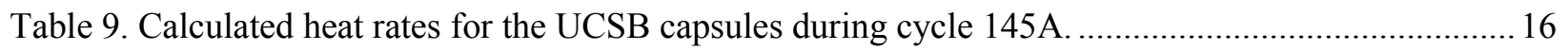

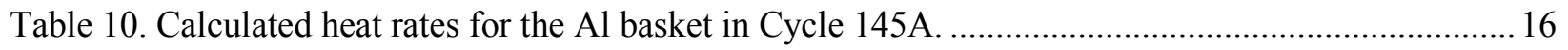

Table 11. Calculated fluence and DPA for Capsule 10 of the UCSB experiment..................................... 18

Table 12. Calculated fluence and DPA for capsule 9 of the UCSB experiment...................................... 22 
Table 13. Calculated fluence and DPA for capsule 8 of the UCSB experiment......................................26

Table 14. Calculated fluence and DPA for capsule 7 of the UCSB experiment...................................... 30

Table 15. Calculated fluence and DPA for capsule 6 of the UCSB experiment...................................... 34

Table 16. Calculated fluence and DPA for capsule 5 of the UCSB experiment...................................... 38

Table 17. Calculated fluence and DPA for Capsule 4 of the UCSB experiment...................................... 42

Table 18. Calculated fluence and DPA for capsule 3 of the UCSB experiment...................................... 46

Table 19. Calculated fluence and DPA for capsule 2 of the UCSB experiment......................................50

Table 20. Calculated fluence and DPA for capsule 2 of the UCSB experiment.........................................5 54

Table 21. Calculated fluence and DPA for capsule 1 of the UCSB experiment.........................................5 


\section{ACRONYMS}

- ATR - Advanced Test Reactor

- MW - Megawatt

- UCSB - University of California Santa Barbara

- DPA - Displacements per atom

- NEFT - Northeast Flux Trap

- NWFT - Northwest Flux Trap

- SEFT - Southeast Flux Trap

- SWFT - Southwest Flux Trap 


\section{As-Run Physics Analysis for the UCSB-1 Experiment in the Advanced Test Reactor}

\section{Introduction}

The purpose of this report is to document the as-run physics analysis for the University of California Santa Barbara (UCSB) -1 experiment in position A-10. The UCSB-1 experiment was irradiated during cycles $145 \mathrm{~A}$ through $146 \mathrm{~B}$. Capsule $6 \mathrm{~A}$ from the test train was removed following cycle $145 \mathrm{~A}$ and replaced with Capsule $6 \mathrm{~B}$

The as-run heat rates, fluence results and DPA were calculated using the MCNP ATR full core model. The heating rates, fluence values were based on ATR operating conditions for the cycles of operation. The DPA was calculated using a 100 group cross-section library as provided in Attachment A. 


\section{Assumptions}

The following assumptions are used in this analysis as stated below:

1. The MCNP models of ATR use three radial fuel regions to represent the ATR fuel elements (FEs)

2. ATR Cycle 145A cycle-averaged lobe powers are reported to be 18.0-17.9-23.2-23.8-25.7 MW (NW-NE-C-SW-SE) (Appendix B).

3. ATR Cycle 145B cycle-averaged lobe powers are reported to be 17.8-17.8-23.0-24.6-25.8 MW (NW-NE-C-SW-SE) (Appendix B).

4. ATR Cycle 146A cycle-averaged lobe powers are reported to be 18.0-18.0-24.3-25.8-26.0 MW (NW-NE-C-SW-SE) (Appendix B).

5. ATR Cycle 146B cycle-averaged lobe powers are reported to be 23.0-18.0-26.0-23.0-26.0 MW (NW-NE-C-SW-SE) (Appendix B).

6. The ATR Cycle 145A as-run cycle length was 54.7 effective full power days (EFPD) (Appendix B)

7. The ATR Cycle 145B as-run cycle length was 57.3 effective full power days (EFPD) (Appendix B)

8. The ATR Cycle 146A as-run cycle length was 50.5 effective full power days (EFPD) (Appendix B)

9. The ATR Cycle 146B as-run cycle length was 39.2 effective full power days (EFPD) (Appendix B)

10. The as-run hourly lobe power history for ATR may be used to scale the MCNP-calculated UCSB heat rate and flux results to represent specific operating conditions for specific times. To scale the UCSB results, the calculated results should be multiplied by the ratio of the desired SE lobe power to the analyzed center lobe power. The scaling equation is defined to be, $\mathrm{HGR}_{\text {desired }}=\mathrm{HGR}_{\text {analyzed }} \times \mathrm{SE}_{\text {desired }}$ / $\mathrm{SE}_{\text {analyzed }}$ (e.g. to scale HGR values at $26 \mathrm{MW}$ SE lobe power to HGR values at $25 \mathrm{MW}$ SE lobe power, $\mathrm{HGR}_{25 \mathrm{MW}}=\mathrm{HGR}_{26 \mathrm{MW}} \times 25 \mathrm{MW} / 26 \mathrm{MW}$ ). This scaling method may also be used to adjust MCNP-calculated power dependent UCSB neutron fluxes. 


\section{Experiment Description}

The objective of the UCSB experiment is to create a large library of irradiated alloys, covering a variety of specimen geometries, to address the following scientific questions [1].

1. The effect of low dose irradiations at many temperatures on the basic microstructures and constitutive properties in disc multi-purpose coupons in different material conditions.

2. The evolution of interface and bulk microstructure, microchemistry and micro-nano hardness in diffusion multiples and bonded and coated specimens.

3. The effects of irradiation at different temperatures on tensile and fracture behaviors of the materials to systematically explore the effects of radiation hardening at lower temperatures and microstructural and phase instabilities at higher temperatures.

4. The stability of the nanoscale Y-Ti-O features (NF) and nonstructured ferritic alloys (NFA) microstructures under high temperature irradiation.

As part of the UCSB experiment different classes of alloys and processing conditions are being investigated, along with nominal compositions of the alloys. The study is focusing on iron-based alloys being considered for next generation reactor applications as both cladding and structural materials and range from simple model alloys to complex commercially fabricated alloys. A listing of the experiment and compositions is presented in Table 1. 


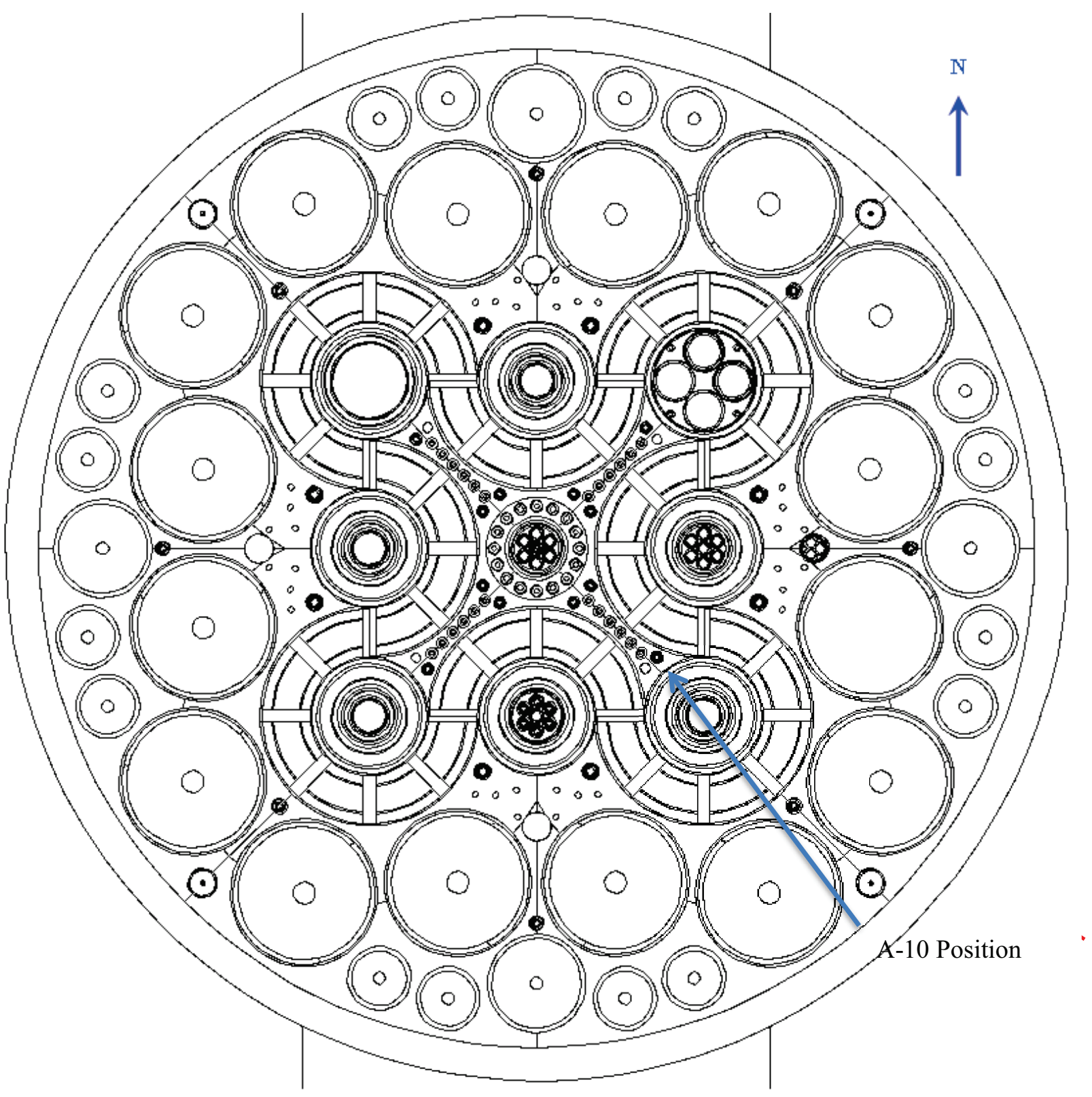

Figure 1. Radial cross section view of the ATR core, A-10 irradiation test position. 
Table 1. Chemical composition of target materials [1].

\begin{tabular}{|c|c|c|c|c|c|c|c|c|c|c|c|c|c|c|c|c|c|c|c|c|c|}
\hline Material ID & $\mathrm{Cr}$ & $\mathbf{T i}$ & Mo & $\mathbf{Y}$ & O & $\mathrm{C}$ & Mn & $\mathbf{S i}$ & $\mathbf{P}$ & $\mathrm{Ni}$ & Al & $\mathbf{S}$ & Sn & $\mathrm{Cu}$ & $\mathbf{W}$ & $\mathbf{N}$ & $\mathbf{N b}$ & $\mathbf{V}$ & B & Co & $\mathrm{Fe}$ \\
\hline T91 & 9.24 & & .96 & & & & 0.47 & 0.28 & & 0.16 & & & & & & & & 0.21 & & & 98.880 \\
\hline HT-9 & 12.2 & & 1.10 & & & 0.21 & 0.32 & 0.29 & 0.002 & 0.57 & 0.010 & & & & 0.51 & & 0.010 & 0.30 & & & 96.678 \\
\hline NF616 & 8.82 & & 0.47 & & & 0.109 & 0.45 & 0.10 & 0.012 & 0.17 & 0.005 & 0.003 & & & 1.87 & 0.047 & 0.064 & 0.19 & 0.0017 & & 87.684 \\
\hline F82H IEA & 7.82 & & & & & 0.090 & 0.10 & 0.07 & 0.003 & 0.02 & & 0.001 & & 0.01 & & & & 0.19 & 0.001 & & 91.695 \\
\hline F82H mod.3 & 8.16 & 0.005 & & & & 0.097 & 0.13 & 0.10 & 0.005 & 0.01 & & & & & 1.98 & 0.007 & & 0.20 & & & 89.306 \\
\hline Eurofer 97 & 8.93 & 0.01 & 0.001 & & & 0.200 & 0.49 & 0.04 & 0.005 & 0.02 & 0.009 & 0.004 & 0.005 & 0.0019 & 1.08 & & 0.0017 & 0.20 & 0.001 & 0.006 & 97.929 \\
\hline MA957 & 13.57 & 0.98 & 0.30 & 0.26 & 0.22 & 0.02 & 0.07 & 0.03 & 0.004 & 0.10 & 0.090 & 0.006 & 0.00 & 0.01 & & & & & & & 84.357 \\
\hline MA956 & 20 & 0.40 & & 0.5 & & 0.1 & 0.3 & & 0.020 & 0.5 & 4.75 & & & 0.15 & & & & & & 0.3 & 72.980 \\
\hline PM2000 & 19.00 & 0.50 & & 0.50 & & & & & & & 5.500 & & & & & & & & & & 74.500 \\
\hline $\begin{array}{l}\text { 14CrYWT- } \\
\text { UCSB1 }\end{array}$ & 14.00 & 0.50 & & 0.25 & & & & & & & & & & & 3.00 & & & & & & 82.250 \\
\hline $\begin{array}{l}\text { 14CrYWT- } \\
\text { UCSB2 }\end{array}$ & 14.00 & 0.50 & & 0.25 & & & & & & & & & & & 3.00 & & & & & & 82.250 \\
\hline Cast SS & 17.71 & & 2.01 & & & 0.010 & 5.14 & 0.45 & 0.300 & 12.60 & & 0.100 & & 2.81 & 1.00 & 0.320 & & & & & 57.550 \\
\hline 9Cr2WYT & 9.01 & 0.23 & & 0.36 & 0.08 & 0.140 & 0.09 & 0.06 & 0.005 & 0.03 & & 0.020 & & & 1.96 & 0.009 & & & & & 88.006 \\
\hline $15 \mathrm{Cr} 2 \mathrm{WYT}$ & 15.00 & 0.20 & & 0.34 & 0.07 & 0.020 & 0.01 & 0.01 & 0.017 & 0.05 & & 0.003 & & & 1.90 & 0.009 & & & & & 82.376 \\
\hline $\begin{array}{l}\text { 14CrYWT } \\
-\mathrm{H}\end{array}$ & 14.50 & 0.23 & 0.03 & 0.23 & 0.16 & 0.048 & 0.52 & 0.05 & 0.008 & 0.02 & 0.002 & 0.002 & 0.01 & 0.005 & 1.04 & 0.012 & 0.001 & 0.21 & & 0.184 & 82.736 \\
\hline $\begin{array}{l}\text { 14CrYWT } \\
\text {-AR }\end{array}$ & 14.29 & 0.23 & 0.03 & 0.22 & 0.15 & 0.069 & 0.66 & 0.04 & 0.008 & 0.03 & 0.003 & 0.002 & 0.01 & 0.007 & 1.06 & 0.027 & 0.001 & 0.22 & & 0.164 & 82.776 \\
\hline $\begin{array}{l}\text { 14CrYWT } \\
\text { ORNL 1 }\end{array}$ & 14.00 & 0.50 & & 0.25 & & & & & & & & & & & 3.00 & & & & & & 82.250 \\
\hline F82H-1.4Ni & & & & & & & & & & 1.40 & & & & & & & & & & & 98.600 \\
\hline $\mathrm{Fe}-3 \mathrm{Cr}$ & 3.30 & & & 0.03 & & 0.01 & & & & & & & & & & 0.003 & & & & & 96.657 \\
\hline $\mathrm{Fe}-6 \mathrm{Cr}$ & 6.00 & & & 0.03 & & 0.01 & & & & & & & & & & 0.003 & & & & & 93.957 \\
\hline $\mathrm{Fe}-9 \mathrm{Cr}$ & 9.60 & & & 0.03 & & 0.01 & & & & & & & & & & 0.003 & & & & & 90.357 \\
\hline $\mathrm{Fe}-12 \mathrm{Cr}$ & 11.60 & & & 0.03 & & 0.01 & & & & & & & & & & 0.003 & & & & & 88.357 \\
\hline $\mathrm{Fe}-15 \mathrm{Cr}$ & 15.10 & & & 0.03 & & 0.01 & & & & & & & & & & 0.003 & & & & & 84.857 \\
\hline $\mathrm{Fe}-18 \mathrm{Cr}$ & 18.30 & & & 0.03 & & 0.01 & & & & & & & & & & 0.003 & & & & & 81.657 \\
\hline $\mathrm{SiC}$ & & & & & & 36.460 & & 63.54 & & & & & & & & & & & & & 0.000 \\
\hline $\begin{array}{l}\text { MAR-1 / } \\
\text { MAR-2 }\end{array}$ & 12.00 & & 1.40 & & & 0.030 & & 0.30 & & 9.20 & 1.600 & & & & & & & & & & 75.470 \\
\hline $\begin{array}{c}\text { Diffusion } \\
\text { Multiple }\end{array}$ & 1.50 & 10.00 & & & & & & & & & & & & & 80.00 & & & & & & 8.500 \\
\hline $\begin{array}{l}\text { Diffusion } \\
\text { Multiple } \\
\end{array}$ & 1.50 & & & & & & & & & 10.00 & & & & & 80.00 & & & & & & 8.500 \\
\hline $\begin{array}{l}\text { Diffusion } \\
\text { Multiple }\end{array}$ & & & & & & & & & & 5.00 & & & & 5.00 & 70.00 & & & & & & 20.000 \\
\hline
\end{tabular}




\begin{tabular}{|c|c|c|c|c|c|c|c|c|c|c|c|c|c|c|c|c|c|c|c|c|c|}
\hline Material ID & $\mathrm{Cr}$ & $\mathbf{T i}$ & Mo & $\mathbf{Y}$ & O & $\mathrm{C}$ & Mn & $\mathrm{Si}$ & $\mathbf{P}$ & $\mathrm{Ni}$ & Al & $\mathbf{S}$ & Sn & $\mathrm{Cu}$ & W & $\mathbf{N}$ & $\mathbf{N b}$ & $\mathbf{V}$ & B & Co & $\mathrm{Fe}$ \\
\hline $\begin{array}{l}\text { Diffusion } \\
\text { Multiple }\end{array}$ & & & & & & & & & & 98.00 & & & & 1.00 & & & 1.00 & & & & 0.000 \\
\hline $\begin{array}{l}\text { Diffusion } \\
\text { Multiple }\end{array}$ & & & & & & & & & & 96.00 & & & & 2.00 & & & 2.00 & & & & 0.000 \\
\hline $\begin{array}{l}\text { Diffusion } \\
\text { Multiple }\end{array}$ & & & & & & & & & & 94.00 & & & & 3.00 & & & 3.00 & & & & 0.000 \\
\hline $\begin{array}{l}\text { Ni Foil } \\
\text { (Pure Element) }\end{array}$ & & & & & & & & & & 100.00 & & & & & & & & & & & 0.000 \\
\hline $\begin{array}{l}\text { Nb Foil } \\
\text { (Pure Element) }\end{array}$ & & & & & & & & & & & & & & & & & 100.00 & & & & 0.000 \\
\hline LA & & & 0.540 & & & 0.140 & 1.370 & 0.220 & 0.005 & & & & & 0.400 & & & & & & & 97.325 \\
\hline LB & & & 0.530 & & & 0.160 & 1.350 & 0.220 & 0.005 & 0.180 & & & & 0.400 & & & & & & & 97.155 \\
\hline $\mathrm{LC}$ & & & 0.550 & & & 0.140 & 1.440 & 0.230 & 0.005 & 0.860 & & & & 0.410 & & & & & & & 96.365 \\
\hline LD & & & 0.530 & & & 0.190 & 1.380 & 0.230 & 0.005 & 1.250 & & & & 0.380 & & & & & & & 96.035 \\
\hline LG & & & 0.550 & & & 0.160 & 1.370 & 0.220 & 0.005 & 0.740 & & & & 0.010 & & & & & & & 96.945 \\
\hline $\mathrm{LH}$ & & & 0.550 & & & 0.160 & 1.390 & 0.240 & 0.005 & 0.740 & & & & 0.110 & & & & & & & 96.805 \\
\hline LI & & & 0.550 & & & 0.160 & 1.370 & 0.240 & 0.005 & 0.740 & & & & 0.200 & & & & & & & 96.735 \\
\hline OV1 & & & & & & & 1.600 & & & & & & & & & & & & & & 98.400 \\
\hline OV2 & & & & & & & 1.600 & & & 0.800 & & & & & & & & & & & 97.600 \\
\hline OV3 & & & & & & & 1.600 & & & 1.600 & & & & & & & & & & & 96.800 \\
\hline OV4 & & & & & & & 1.600 & & & 0.800 & & & & 0.050 & & & & & & & 97.550 \\
\hline OV5 & & & & & & & 1.600 & & & 1.600 & & & & 0.050 & & & & & & & 96.750 \\
\hline OV6 & & & & & & & 1.600 & & & 0.800 & & & & 0.100 & & & & & & & 97.500 \\
\hline OV7 & & & & & & & 1.600 & & & 1.600 & & & & 0.100 & & & & & & & 96.700 \\
\hline CM6 & & & 0.540 & & & 0.150 & 1.550 & 0.170 & 0.007 & 1.680 & & & & 0.020 & & & & & & & 95.883 \\
\hline JRQ & 0.120 & & 0.500 & & & 0.180 & 1.400 & 0.250 & 0.019 & 0.820 & & 0.004 & & 0.14 & & & & 0.003 & & & 96.564 \\
\hline $430 \mathrm{SS}$ & 18.000 & & & & & 0.120 & 1.000 & 1.000 & & & & & & & & & & & & & 79.880 \\
\hline
\end{tabular}


Most of the specimens used for the UCSB experiment are simple DMC samples $(0.2 \mathrm{~mm}$ thick and $\sim 10 \mathrm{~mm}$ in diameter) that will be used for microstructural studies (TEM, SANS, etc...) to study constitutive properties by advanced hardness techniques, shear punch tests (also making TEM discs), post irradiation annealing studies and disc bend (DB) fracture tests. Selected alloys are included in the form of sub-sized SS-J tensile specimens, disc tensile specimen (DTS), disc compact tension (DCT) fracture specimens, and deformation and fracture minibeams (DFMB). The DM specimens, made of three to four joined elements (or different alloys), are used to study radiation modified inter-diffusion and phase formation processes, encompassing the entire phase diagram at the specified temperature. Some advanced sub-sized dual chevron notched cantilevered beam specimens and dualnotched disc (DCD) specimens are included. A summary of the samples used in the UCSB experiment can be found in Attachment B. The samples selected were inserted into the irradiation test assembly with target temperatures and displacements per atom (dpa) shown in Table 2.

Table 2. Target temperature and dose for each UCSB capsule [1].

\begin{tabular}{|c|c|c|}
\hline Capsule ID & $\begin{array}{c}\text { Target } \\
\text { Temperature }\end{array}$ & $\begin{array}{c}\text { Nominal Target } \\
\text { Dose }\end{array}$ \\
\hline UCSB-1 & $275{ }^{\circ} \mathrm{C}$ & $1.7 \mathrm{dpa}$ \\
\hline UCSB-2 & $550-750^{\circ} \mathrm{C}$ & 2.4 to $4.2 \mathrm{dpa}$ \\
\hline UCSB-3 & $350-550{ }^{\circ} \mathrm{C}$ & $4.5-5.1 \mathrm{dpa}$ \\
\hline UCSB-4 & $650-750^{\circ} \mathrm{C}$ & $5.5-5.8 \mathrm{dpa}$ \\
\hline UCSB-5 & $350-500{ }^{\circ} \mathrm{C}$ & $6.0-6.2 \mathrm{dpa}$ \\
\hline UCSB-6A* & $275^{\circ} \mathrm{C}$ & $1.7 \mathrm{dpa}$ \\
\hline UCSB-6B* & $275^{\circ} \mathrm{C}$ & $4.6 \mathrm{dpa}$ \\
\hline UCSB-7 & $275^{\circ} \mathrm{C}$ & $6.0-6.2 \mathrm{dpa}$ \\
\hline UCSB-8 & $275^{\circ} \mathrm{C}$ & $5.6-5.7 \mathrm{dpa}$ \\
\hline UCSB-9 & $275^{\circ} \mathrm{C}$ & $4.2-5.2 \mathrm{dpa}$ \\
\hline UCSB-10 & $350-500{ }^{\circ} \mathrm{C}$ & $1.8-3.8 \mathrm{dpa}$ \\
\hline
\end{tabular}

The stainless steel capsule assembly contain a vertical stack of sample packets with top and bottom caps for packet separation. The sample packets consist of samples rigidly fixed by aligning pins or cylindrical sample holders. The sample holders are machined from thermally conductive metal capable of withstanding the designed irradiation temperature. The endcaps are made of stainless steel with the sample packet on the bottom of the capsule sitting partially within the endcap.

A total of ten capsule assemblies were used in the UCSB experiment. Capsule 6A was removed after the first cycle and exchanged with a new capsule (6B) containing new specimens. A schematic of the capsule configuration is shown in Figure 2 and Table 3 gives the total length of each capsule. The test assembly that was used for the UCSB experiment was made of aluminum and designed to interface the capsule assemblies with the ATR A-10 position. 


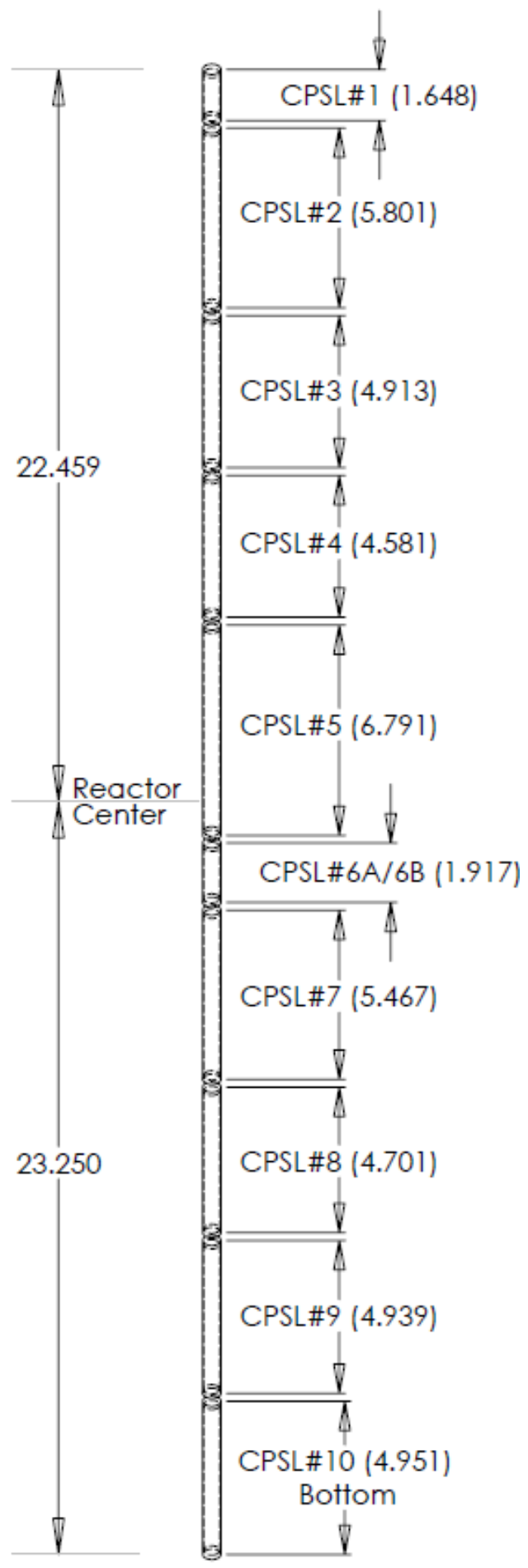

Figure 2. Capsule assembly schematic (lengths are in inches) [1]. 
Table 3. Capsule lengths for UCSB experiment [1].

\begin{tabular}{|c|c|}
\hline Capsule & Length (in) \\
\hline 1 & 1.648 \\
\hline 2 & 5.801 \\
\hline 3 & 4.913 \\
\hline 4 & 4.581 \\
\hline 5 & 6.791 \\
\hline $6 \mathrm{~A} / 6 \mathrm{~B}$ & 1.917 \\
\hline 7 & 5.467 \\
\hline 8 & 4.701 \\
\hline 9 & 4.939 \\
\hline 10 & 4.951 \\
\hline
\end{tabular}




\section{Model Description and Data}

MCNP [2][3], a general purpose $\underline{\text { Monte }}$ Carlo $\underline{N}-\underline{P}$ article transport code, was used to model and evaluate the UCSB experiment. A full core 3D model of the ATR was used for analyses.

The model for the UCSB experiment is based on the drawings listed in Table 4 and the Irradiation Test Plan for UCSB [1]. Nominal dimensions for the samples, sample holders, and capsules are used in the model. The experiment housing dimensions were obtained from existing INL drawings. Table 5 provides a summary of the dimensions used to model the UCSB experiment. The sample packet lengths were based on information provided in the irradiation test plan [1].

Table 4. List of drawings for UCSB experiment.

\begin{tabular}{|cl|}
\hline INL Drawing & \multicolumn{1}{c|}{ Drawing Title } \\
\hline 760173 & $\begin{array}{l}\text { ATR NSUF UNIVERSITY OF CALIFORNIA SANTA BARBARA A-HOLE } \\
\text { OUTER CAPSULE ASSEMBLIES }\end{array}$ \\
\hline 760174 & $\begin{array}{l}\text { ATR NSUF UNIVERSITY OF CALIFORNIA SANTA BARBRA BASKET } \\
\text { ASSEMBLIES }\end{array}$ \\
\hline
\end{tabular}

Table 5. Radial dimensions for modeling the UCSB experiment.

\begin{tabular}{|l|c|}
\hline Design Parameter & $\begin{array}{c}\text { Radius } \\
(\mathbf{c m})\end{array}$ \\
\hline Specimen Packet & 0.500 \\
\hline Gas Fill & 0.546 \\
\hline Capsule Outer Radius & 0.610 \\
\hline
\end{tabular}

\subsection{Data Libraries}

The standard MCNP cross-section data libraries [3] were used to calculate flux and heating rates for the UCSB experiment. 


\section{Analysis and Calculations}

MCNP is used to calculate the heat generation rate and flux in the UCSB experiment. MCNP reports the flux tallies in units of $1 / \mathrm{cm}^{2}$ per source neutron. The following normalization factors are used to calculate a neutron flux and the heat generation rates from the MCNP tallies.

Neutron Flux Normalization Factor

Note, the MCNP f4 tally has units of $1 / \mathrm{cm}^{2}$ per source neutron.

$\left(\frac{\text { fission neutrons }}{\text { fission }}\right)\left(\frac{\text { fission }}{M e V}\right)\left(\frac{M e V}{M W_{\text {core power }}-s}\right)=$ Flux Normalization

$\left(\frac{2.43 \text { fission neutrons }}{\text { fission }}\right)\left(\frac{\text { fission }}{200 \mathrm{MeV}}\right)\left(\frac{6.24146 \times 10^{18} \mathrm{MeV}}{M W_{\text {core power }}-s}\right)=7.583 \times 10^{16} \frac{\text { fission neutrons }}{M W_{\text {core power }}-s}$

\section{Displacements per Atom}

The DPA calculations were performed using the flux provided as described above multiplied by a 100 group cross-section for DPA (see Attachment B). The DPA is then calculated by the following formula:

$$
\left(\phi \frac{\text { neutrons }}{\mathrm{cm}^{2}-\mathrm{sec}}\right)(\sigma(\mathrm{keV}-\mathrm{barns}))\left(\frac{1000 \mathrm{eV}}{\mathrm{keV}}\right) \frac{1}{2 * 40 \mathrm{eV}} * \frac{1 \times 10^{-24} \mathrm{~cm}^{2}}{b a r n}=\mathrm{dpa} / \mathrm{sec}
$$

Neutron/Prompt Gamma Heating Normalization Factor (NHNF)

Note, MCNP f6 or $\mathrm{f} 7$ tally has units of $\mathrm{MeV} / \mathrm{g}$ per source neutron.

$$
\mathrm{NHNF}=\left(\frac{\text { fission neutrons }}{\text { fission }}\right)\left(\frac{\text { fission }}{\mathrm{MeV}}\right)\left(\frac{\mathrm{MeV}}{\mathrm{MW}_{\text {core power }}-\mathrm{s}}\right)\left(\frac{\mathrm{W}-\mathrm{s}}{\mathrm{MeV}}\right)
$$

$=\left(\frac{7.583 \times 10^{16} \text { fission neutrons }}{M W_{\text {core power }}-s}\right)\left(\frac{1.60219 \times 10^{-13} W-s}{M e V}\right)$

$=\left(1.215 \times 10^{4} \frac{\text { fission neutrons }-W}{M W_{\text {core power }}-M e V}\right)$

Delayed Fission Product Gamma Heating Normalization Factor

Note, the MCNP f6 tally has units of MeV/g per source photon.

$\left(\frac{\text { delayedfissionphotons }}{\text { fission }}\right)\left(\frac{\text { fission }}{\mathrm{MeV}}\right)\left(\frac{\mathrm{MeV}}{\mathrm{MW}_{\text {corepower }}-\mathrm{s}}\right)\left(\frac{\mathrm{W}-\mathrm{s}}{\mathrm{MeV}}\right)=$ HeatNormalizaiton 


$$
\begin{gathered}
\left(\frac{8.9603 \text { delayed fission photons }}{\text { fission }}\right)\left(\frac{\text { fission }}{200 \mathrm{MeV}}\right)\left(\frac{6.24146 \times 10^{18} \mathrm{MeV}}{M W_{\text {core power }}-s}\right)\left(\frac{1.60219 \times 10^{-13} \mathrm{~W}-s}{M e V}\right)= \\
4.48015 \times 10^{4} \frac{\text { delyed fission photons }-W}{M W_{\text {core power }}-M e V}
\end{gathered}
$$




\section{Software}

The computer codes MCNP and ORIGEN2 are listed in the INL Enterprise Architecture (EA) Repository and are accepted as qualified scientific and engineering analysis software. Table 6 lists the version and EA ID for the computer codes used to perform the calculations and analyses documented by this ECAR.

Table 6. INL Qualified Analysis Software, Version, and EA ID.

\begin{tabular}{|l|l|l|}
\hline Code Name & Version & EA ID \\
\hline MCNP & 5 (Release 1.40) & $234166[5]$ \\
\hline
\end{tabular}

MCNP has been verified and validated (V\&V'd) for use at the INL as documented by the MCNP Version 5, Release 1.40 software management report [5]. The MCNP Version 5, Release $1.40 \mathrm{~V} \& \mathrm{~V}$ process was performed and accepted on high performance computing (HPC) systems at the INL. The computer configurations listed in Table 7 were used to perform the MCNP5.

Table 7: Computer Configurations for INL Qualified MCNP5 installations.

\begin{tabular}{|c|c|c|}
\hline Model of Computer & Processor & Operating System \\
\hline $\begin{array}{l}\text { fission is an Appro } \\
\text { (Xtreme-X } \mathrm{X}^{\mathrm{TM}} \\
\text { Supercomputer Series) } \\
\text { distributed memory } \\
\text { cluster }\end{array}$ & $\begin{array}{l}\text { Two service nodes acting as login } \\
\text { nodes each with: } \\
\text { - Two } 8 \text { core } 2.4 \mathrm{GHz} \text { AMD Opteron } \\
\text { (6136) processors } \\
\text { - } 32 \mathrm{~GB} \text { of shared memory ( } 2 \mathrm{~GB} / \text { core) } \\
391 \text { compute blades with: } \\
\text { - Four } 8 \text { core } 2.4 \mathrm{GHz} \text { AMD Opteron } \\
\text { (6136) processors per blade ( } 32 \\
\text { cores/node and } 12512 \text { cores total) } \\
\text { - } 64 \text { GB of shared memory per node ( } 2 \\
\text { GB/core) } \\
\text { QDR InfiniBand interconnect network }\end{array}$ & $\begin{array}{l}\text { RedHat Linux Enterprise } \\
\text { Server } 5.5\end{array}$ \\
\hline
\end{tabular}




\section{Analysis Results}

The MCNP full core physics model was used to calculate as-run heat rates, flux and fluence for the UCSB experiment. The as-run flux was used to determine the DPA for the experiment based on the irradiation time. The calculated heat rates for cycle $145 \mathrm{~A}$ is provided in Table 8 through Table 10. The heating rates for subsequent cycles can be scaled by their respective cycle powers from the analyzed cycle power in 145A of $25.70 \mathrm{MW}$. The lobe powers for 145B, 146A, and 146B are 25.8 MW, 26.0 MW and 26.0 MW, which results in a scaling factor of $1.004,1.011$, and 1.011 for cycles $145 \mathrm{~B}, 146 \mathrm{~A}$, and 146B, respectively.

The calculated fluence and DPA were determined from the ATR MCNP model and as-run cycle data. The results for each packet are reported in Table 11 through Table 21. The DPA results are reported based on Fe damage cross-sections provide in Attachment B. 
Table 8. Calculated as run heating rates for UCSB packets for Cycle 145A

\begin{tabular}{|c|c|c|c|c|c|c|}
\hline Target & $\begin{array}{c}\text { Core Elevation (inches from } \\
\text { bottom of core) }\end{array}$ & Segment Length (inches) & Mass (grams) & Neutron Heating (Watts/gram) & Photon Heating (Watts/gram) & Heat Generation (Watts/gram) \\
\hline packet $10-4$ & 1.239 & 0.728 & 11.268 & 0.044 & 3.893 & 3.936 \\
\hline packet $10-3$ & 2.007 & 0.807 & 12.370 & 0.049 & 4.335 & 4.384 \\
\hline packet $10-2$ & 2.893 & 0.965 & 14.905 & 0.048 & 4.798 & 4.846 \\
\hline packet 10-1 & 3.857 & 0.965 & 14.873 & 0.052 & 5.352 & 5.403 \\
\hline packet $9-4$ & 6.259 & 0.866 & 13.313 & 0.066 & 6.683 & 6.749 \\
\hline packet 9-3 & 7.273 & 1.161 & 18.320 & 0.072 & 7.947 & 8.019 \\
\hline packet 9-2 & 8.515 & 1.323 & 20.503 & 0.085 & 7.916 & 8.001 \\
\hline packet 9-1 & 9.649 & 0.945 & 14.727 & 0.115 & 8.294 & 8.409 \\
\hline packet 8-1 & 12.893 & 4.257 & 70.901 & 0.115 & 9.328 & 9.444 \\
\hline packet 7-3 & 16.214 & 1.496 & 23.233 & 0.105 & 10.134 & 10.238 \\
\hline packet 7-2 & 17.208 & 0.492 & 7.560 & 0.092 & 10.185 & 10.276 \\
\hline packet 7-1 & 18.872 & 2.835 & 43.813 & 0.109 & 10.486 & 10.595 \\
\hline packet $6 \mathrm{~A}-1$ & 21.563 & 1.260 & 19.489 & 0.104 & 10.611 & 10.714 \\
\hline packet 5-4 & 23.628 & 1.555 & 24.133 & 0.116 & 10.658 & 10.773 \\
\hline packet 5-3 & 25.055 & 1.299 & 20.052 & 0.116 & 10.551 & 10.667 \\
\hline packet 5-2 & 26.403 & 1.398 & 21.660 & 0.115 & 10.461 & 10.576 \\
\hline packet 5-1 & 27.811 & 1.417 & 21.909 & 0.112 & 10.399 & 10.511 \\
\hline packet 4-3 & 30.360 & 1.437 & 21.430 & 0.113 & 10.191 & 10.304 \\
\hline packet 4-2 & 31.816 & 1.476 & 22.497 & 0.098 & 9.772 & 9.870 \\
\hline packet 4-1 & 32.987 & 0.866 & 13.315 & 0.084 & 9.393 & 9.477 \\
\hline packet 3-4 & 35.255 & 2.067 & 32.057 & 0.100 & 8.797 & 8.897 \\
\hline packet 3-3 & 36.722 & 0.866 & 13.448 & 0.080 & 8.087 & 8.167 \\
\hline packet 3-2 & 37.454 & 0.598 & 9.247 & 0.082 & 7.819 & 7.901 \\
\hline packet 3-1 & 38.039 & 0.571 & 9.240 & 0.074 & 8.854 & 8.928 \\
\hline packet 2-7 & 39.568 & 0.866 & 13.328 & 0.069 & 6.907 & 6.975 \\
\hline packet 2-6 & 40.346 & 0.689 & 11.562 & 0.066 & 7.826 & 7.891 \\
\hline packet 2-5 & 40.936 & 0.492 & 7.430 & 0.062 & 6.227 & 6.288 \\
\hline packet 2-4 & 41.428 & 0.492 & 7.408 & 0.058 & 5.913 & 5.971 \\
\hline packet 2-3 & 42.078 & 0.807 & 12.305 & 0.059 & 5.483 & 5.542 \\
\hline packet 2-2 & 42.737 & 0.512 & 7.719 & 0.047 & 5.061 & 5.108 \\
\hline
\end{tabular}




\begin{tabular}{|c|c|c|c|c|c|c|}
\hline Target & $\begin{array}{c}\text { Core Elevation (inches from } \\
\text { bottom of core) }\end{array}$ & Segment Length (inches) & Mass (grams) & Neutron Heating (Watts/gram) & Photon Heating (Watts/gram) & Heat Generation (Watts/gram) \\
\hline packet 2-1 & 43.239 & 0.492 & 7.417 & 0.044 & 4.679 & 4.723 \\
\hline packet $1-1$ & 45.438 & 1.004 & 15.507 & 0.033 & 3.451 & 3.484 \\
\hline
\end{tabular}

Table 9. Calculated heat rates for the UCSB capsules during cycle 145A.

\begin{tabular}{|c|c|c|c|c|c|c|}
\hline Segment & Core Elevation (inches from bottom of core) & Segment Length (inches) & Mass (grams) & Neutron Heating (Watts/gram) & Photon Heating (Watts/gram) & Heat Generation (Watts/gram) \\
\hline 1 & 3.226 & 4.951 & 27.357 & 0.046 & 5.387 & 5.434 \\
\hline 2 & 8.171 & 4.939 & 27.294 & 0.073 & 8.194 & 8.267 \\
\hline 3 & 12.991 & 4.701 & 26.193 & 0.090 & 10.047 & 10.137 \\
\hline 4 & 18.075 & 5.467 & 29.717 & 0.099 & 11.104 & 11.203 \\
\hline 5 & 21.767 & 1.917 & 13.432 & 0.101 & 11.308 & 11.409 \\
\hline 6 & 26.121 & 6.791 & 35.795 & 0.100 & 11.240 & 11.340 \\
\hline 7 & 31.807 & 4.581 & 25.642 & 0.093 & 10.348 & 10.441 \\
\hline 8 & 36.554 & 4.913 & 27.175 & 0.079 & 8.783 & 8.863 \\
\hline 9 & 41.911 & 5.801 & 31.259 & 0.053 & 5.941 & 5.994 \\
\hline 10 & 45.635 & 1.648 & 12.188 & 0.031 & 3.538 & 3.569 \\
\hline
\end{tabular}

Table 10. Calculated heat rates for the Al basket in Cycle 145A.

\begin{tabular}{|c|c|c|c|c|c|c|}
\hline Segment & Core Elevation (inches from bottom of core) & Segment Length (inches) & Mass (grams) & Neutron Heating (Watts/gram) & Photon Heating (Watts/gram) & Heat Generation (Watts/gram) \\
\hline 1 & -7.375 & 17.958 & 48.360 & 0.019 & 0.937 & 0.956 \\
\hline 2 & 2.007 & 0.807 & 2.173 & 0.128 & 3.827 & 3.955 \\
\hline 3 & 2.893 & 0.965 & 2.598 & 0.142 & 4.226 & 4.368 \\
\hline 4 & 3.857 & 0.965 & 2.598 & 0.157 & 4.740 & 4.897 \\
\hline 5 & 5.516 & 2.353 & 6.335 & 0.189 & 5.577 & 5.766 \\
\hline 6 & 7.273 & 1.161 & 3.128 & 0.224 & 6.427 & 6.651 \\
\hline 7 & 8.515 & 1.323 & 3.562 & 0.241 & 6.948 & 7.189 \\
\hline 8 & 9.649 & 0.945 & 2.545 & 0.253 & 7.351 & 7.604 \\
\hline 9 & 12.571 & 4.900 & 13.197 & 0.283 & 8.179 & 8.462 \\
\hline 10 & 15.992 & 1.940 & 5.226 & 0.306 & 8.898 & 9.203 \\
\hline 11 & 17.208 & 0.492 & 1.325 & 0.310 & 9.008 & 9.318 \\
\hline 12 & 18.872 & 2.835 & 7.634 & 0.315 & 9.194 & 9.509 \\
\hline
\end{tabular}




\begin{tabular}{|c|c|c|c|c|c|c|}
\hline Segment & Core Elevation (inches from bottom of core) & Segment Length (inches) & Mass (grams) & Neutron Heating (Watts/gram) & Photon Heating (Watts/gram) & Heat Generation (Watts/gram) \\
\hline 13 & 21.241 & 1.904 & 5.127 & 0.321 & 9.345 & 9.665 \\
\hline 14 & 23.299 & 2.212 & 5.957 & 0.320 & 9.331 & 9.651 \\
\hline 15 & 25.055 & 1.299 & 3.499 & 0.319 & 9.234 & 9.553 \\
\hline 16 & 26.403 & 1.398 & 3.764 & 0.318 & 9.177 & 9.495 \\
\hline 17 & 27.811 & 1.417 & 3.817 & 0.315 & 9.119 & 9.433 \\
\hline 18 & 29.799 & 2.559 & 6.890 & 0.305 & 8.935 & 9.240 \\
\hline 19 & 31.816 & 1.476 & 3.976 & 0.292 & 8.489 & 8.781 \\
\hline 20 & 32.987 & 0.866 & 2.332 & 0.286 & 8.274 & 8.560 \\
\hline 21 & 34.855 & 2.868 & 7.725 & 0.272 & 7.836 & 8.108 \\
\hline 22 & 36.722 & 0.866 & 2.332 & 0.250 & 7.156 & 7.406 \\
\hline 23 & 37.454 & 0.598 & 1.612 & 0.239 & 6.905 & 7.145 \\
\hline 24 & 38.039 & 0.571 & 1.537 & 0.233 & 6.667 & 6.900 \\
\hline 25 & 39.163 & 1.677 & 4.515 & 0.221 & 6.318 & 6.540 \\
\hline 26 & 40.346 & 0.689 & 1.855 & 0.205 & 5.758 & 5.962 \\
\hline 27 & 40.936 & 0.492 & 1.325 & 0.192 & 5.458 & 5.650 \\
\hline 28 & 41.428 & 0.492 & 1.325 & 0.181 & 5.204 & 5.385 \\
\hline 29 & 42.078 & 0.807 & 2.173 & 0.164 & 4.831 & 4.995 \\
\hline 30 & 42.737 & 0.512 & 1.378 & 0.148 & 4.410 & 4.558 \\
\hline 31 & 43.239 & 0.492 & 1.325 & 0.137 & 4.128 & 4.265 \\
\hline 32 & 44.713 & 2.455 & 6.609 & 0.112 & 3.490 & 3.602 \\
\hline 33 & 55.647 & 19.414 & 52.283 & 0.015 & 0.638 & 0.653 \\
\hline
\end{tabular}


Table 11. Calculated fluence and DPA for Capsule 10 of the UCSB experiment.

\begin{tabular}{|c|c|c|c|c|c|c|c|c|c|}
\hline \multirow[b]{2}{*}{ Upper Energy(MeV) } & \multirow{2}{*}{$\begin{array}{l}\text { DPA xsec } \\
\text { keV-barn }\end{array}$} & \multicolumn{2}{|c|}{$10-4$} & \multicolumn{2}{|c|}{$10-3$} & \multicolumn{2}{|c|}{$10-2$} & \multicolumn{2}{|c|}{$10-1$} \\
\hline & & Total Fluence & DPA & Total Fluence & DPA & Total Fluence & DPA & Total Fluence & DPA \\
\hline $1.00 \mathrm{E}-10$ & & $1.92 \mathrm{E}+14$ & & $0.00 E+00$ & & $3.57 \mathrm{E}+14$ & & $3.67 E+15$ & \\
\hline $1.00 \mathrm{E}-09$ & 7.73 & $2.35 \mathrm{E}+17$ & 2.27E-05 & $4.89 \mathrm{E}+17$ & 4.73E-05 & $2.83 \mathrm{E}+17$ & 2.73E-05 & $4.06 \mathrm{E}+17$ & $3.92 \mathrm{E}-05$ \\
\hline $1.00 \mathrm{E}-08$ & 2.444 & $5.34 \mathrm{E}+19$ & $1.63 \mathrm{E}-03$ & $5.28 \mathrm{E}+19$ & $1.61 \mathrm{E}-03$ & $5.57 \mathrm{E}+19$ & $1.70 \mathrm{E}-03$ & $6.39 E+19$ & $1.95 \mathrm{E}-03$ \\
\hline $2.30 \mathrm{E}-08$ & 1.278 & $1.94 \mathrm{E}+20$ & $3.09 \mathrm{E}-03$ & $1.93 E+20$ & $3.08 \mathrm{E}-03$ & $2.02 \mathrm{E}+20$ & $3.23 \mathrm{E}-03$ & $2.31 E+20$ & $3.68 \mathrm{E}-03$ \\
\hline $5.00 \mathrm{E}-08$ & 0.857 & $4.77 \mathrm{E}+20$ & $5.11 \mathrm{E}-03$ & $4.76 \mathrm{E}+20$ & $5.10 \mathrm{E}-03$ & $5.14 \mathrm{E}+20$ & 5.50E-03 & $5.67 E+20$ & 6.07E-03 \\
\hline $7.60 \mathrm{E}-08$ & 0.644 & $3.27 E+20$ & 2.63E-03 & $3.31 E+20$ & $2.66 \mathrm{E}-03$ & $3.66 \mathrm{E}+20$ & $2.95 \mathrm{E}-03$ & $4.09 E+20$ & $3.29 \mathrm{E}-03$ \\
\hline $1.15 \mathrm{E}-07$ & 0.523 & $2.74 \mathrm{E}+20$ & $1.79 \mathrm{E}-03$ & $2.80 \mathrm{E}+20$ & $1.83 \mathrm{E}-03$ & $3.17 \mathrm{E}+20$ & 2.07E-03 & $3.51 E+20$ & 2.29E-03 \\
\hline $1.70 \mathrm{E}-07$ & 0.428 & $1.64 \mathrm{E}+20$ & 8.75E-04 & $1.72 \mathrm{E}+20$ & $9.21 \mathrm{E}-04$ & $1.92 \mathrm{E}+20$ & $1.03 \mathrm{E}-03$ & $2.14 \mathrm{E}+20$ & $1.14 \mathrm{E}-03$ \\
\hline $2.55 \mathrm{E}-07$ & 0.351 & $1.08 \mathrm{E}+20$ & 4.75E-04 & $1.22 \mathrm{E}+20$ & $5.36 \mathrm{E}-04$ & $1.34 \mathrm{E}+20$ & 5.89E-04 & $1.54 \mathrm{E}+20$ & 6.76E-04 \\
\hline $3.80 \mathrm{E}-07$ & 0.287 & $8.80 E+19$ & 3.16E-04 & $9.83 E+19$ & 3.53E-04 & $1.08 \mathrm{E}+20$ & 3.87E-04 & $1.30 \mathrm{E}+20$ & $4.68 \mathrm{E}-04$ \\
\hline $5.50 \mathrm{E}-07$ & 0.237 & $8.22 \mathrm{E}+19$ & $2.43 \mathrm{E}-04$ & $9.19 E+19$ & $2.72 \mathrm{E}-04$ & $1.02 \mathrm{E}+20$ & 3.04E-04 & $1.16 \mathrm{E}+20$ & $3.44 \mathrm{E}-04$ \\
\hline $8.40 \mathrm{E}-07$ & 0.194 & $8.76 \mathrm{E}+19$ & $2.12 \mathrm{E}-04$ & $9.61 \mathrm{E}+19$ & $2.33 \mathrm{E}-04$ & $1.13 \mathrm{E}+20$ & $2.74 \mathrm{E}-04$ & $1.26 \mathrm{E}+20$ & $3.05 \mathrm{E}-04$ \\
\hline $1.28 \mathrm{E}-06$ & 0.157 & $8.40 \mathrm{E}+19$ & $1.65 \mathrm{E}-04$ & $9.72 \mathrm{E}+19$ & $1.91 \mathrm{E}-04$ & $1.12 \mathrm{E}+20$ & $2.20 \mathrm{E}-04$ & $1.25 \mathrm{E}+20$ & $2.45 \mathrm{E}-04$ \\
\hline $1.90 \mathrm{E}-06$ & 0.128 & 8.19E+19 & $1.31 \mathrm{E}-04$ & $9.32 \mathrm{E}+19$ & $1.49 \mathrm{E}-04$ & $9.92 \mathrm{E}+19$ & $1.59 \mathrm{E}-04$ & $1.18 \mathrm{E}+20$ & $1.89 \mathrm{E}-04$ \\
\hline $2.80 \mathrm{E}-06$ & 0.105 & $7.74 \mathrm{E}+19$ & $1.02 \mathrm{E}-04$ & $9.13 E+19$ & $1.20 \mathrm{E}-04$ & $1.01 \mathrm{E}+20$ & $1.33 \mathrm{E}-04$ & $1.12 \mathrm{E}+20$ & 1.47E-04 \\
\hline 4.25E-06 & 0.086 & $8.40 \mathrm{E}+19$ & $9.02 \mathrm{E}-05$ & $9.49 \mathrm{E}+19$ & $1.02 \mathrm{E}-04$ & $1.05 \mathrm{E}+20$ & $1.13 \mathrm{E}-04$ & $1.17 \mathrm{E}+20$ & $1.26 \mathrm{E}-04$ \\
\hline $6.30 \mathrm{E}-06$ & 0.07 & $7.85 E+19$ & 6.87E-05 & $8.90 \mathrm{E}+19$ & 7.79E-05 & $9.69 \mathrm{E}+19$ & $8.48 \mathrm{E}-05$ & $1.10 \mathrm{E}+20$ & 9.67E-05 \\
\hline $9.20 \mathrm{E}-06$ & 0.058 & $7.56 \mathrm{E}+19$ & $5.48 \mathrm{E}-05$ & $8.67 \mathrm{E}+19$ & $6.28 \mathrm{E}-05$ & $9.73 E+19$ & 7.06E-05 & $1.07 \mathrm{E}+20$ & 7.76E-05 \\
\hline $1.35 \mathrm{E}-05$ & 0.048 & $7.75 \mathrm{E}+19$ & 4.65E-05 & $8.83 \mathrm{E}+19$ & $5.30 \mathrm{E}-05$ & $1.02 \mathrm{E}+20$ & 6.10E-05 & $1.14 \mathrm{E}+20$ & $6.87 \mathrm{E}-05$ \\
\hline 2.10E-05 & 0.038 & $9.08 \mathrm{E}+19$ & 4.31E-05 & $1.02 \mathrm{E}+20$ & 4.86E-05 & $1.17 \mathrm{E}+20$ & $5.58 \mathrm{E}-05$ & $1.29 \mathrm{E}+20$ & $6.12 \mathrm{E}-05$ \\
\hline $3.00 \mathrm{E}-05$ & 0.031 & $7.25 \mathrm{E}+19$ & $2.81 \mathrm{E}-05$ & $8.20 \mathrm{E}+19$ & $3.18 \mathrm{E}-05$ & $9.50 \mathrm{E}+19$ & $3.68 \mathrm{E}-05$ & $1.05 \mathrm{E}+20$ & 4.08E-05 \\
\hline $4.50 \mathrm{E}-05$ & 0.026 & $7.59 \mathrm{E}+19$ & 2.47E-05 & $9.15 \mathrm{E}+19$ & $2.98 \mathrm{E}-05$ & $1.08 \mathrm{E}+20$ & 3.49E-05 & $1.24 \mathrm{E}+20$ & 4.01E-05 \\
\hline $6.90 \mathrm{E}-05$ & 0.021 & $8.78 \mathrm{E}+19$ & $2.31 \mathrm{E}-05$ & $1.01 \mathrm{E}+20$ & $2.64 \mathrm{E}-05$ & $1.17 \mathrm{E}+20$ & 3.06E-05 & $1.29 \mathrm{E}+20$ & 3.39E-05 \\
\hline $1.00 \mathrm{E}-04$ & 0.017 & $8.06 \mathrm{E}+19$ & $1.71 \mathrm{E}-05$ & $8.95 \mathrm{E}+19$ & $1.90 \mathrm{E}-05$ & $1.05 \mathrm{E}+20$ & $2.23 \mathrm{E}-05$ & $1.13 \mathrm{E}+20$ & $2.40 \mathrm{E}-05$ \\
\hline $1.35 \mathrm{E}-04$ & 0.014 & $6.38 \mathrm{E}+19$ & $1.12 \mathrm{E}-05$ & $7.22 E+19$ & $1.26 \mathrm{E}-05$ & $8.09 E+19$ & $1.42 \mathrm{E}-05$ & $9.38 \mathrm{E}+19$ & $1.64 \mathrm{E}-05$ \\
\hline $1.70 \mathrm{E}-04$ & 0.014 & $4.71 \mathrm{E}+19$ & $8.24 \mathrm{E}-06$ & $5.36 \mathrm{E}+19$ & $9.38 \mathrm{E}-06$ & $6.56 \mathrm{E}+19$ & $1.15 \mathrm{E}-05$ & $7.15 E+19$ & $1.25 \mathrm{E}-05$ \\
\hline $2.20 \mathrm{E}-04$ & 0.011 & $5.41 \mathrm{E}+19$ & 7.44E-06 & $6.17 \mathrm{E}+19$ & $8.48 \mathrm{E}-06$ & $7.04 \mathrm{E}+19$ & $9.68 \mathrm{E}-06$ & $8.47 \mathrm{E}+19$ & 1.16E-05 \\
\hline $2.80 \mathrm{E}-04$ & 0.011 & $5.11 E+19$ & 7.03E-06 & $6.35 E+19$ & 8.73E-06 & $6.93 E+19$ & $9.53 \mathrm{E}-06$ & $7.63 E+19$ & $1.05 \mathrm{E}-05$ \\
\hline
\end{tabular}




\begin{tabular}{|c|c|c|c|c|c|c|c|c|c|}
\hline \multirow[b]{2}{*}{ Upper Energy(MeV) } & \multirow{2}{*}{$\begin{array}{l}\text { DPA xsec } \\
\text { keV-barn }\end{array}$} & \multicolumn{2}{|c|}{$10-4$} & \multicolumn{2}{|c|}{$10-3$} & \multicolumn{2}{|c|}{$10-2$} & \multicolumn{2}{|c|}{$10-1$} \\
\hline & & Total Fluence & DPA & Total Fluence & DPA & Total Fluence & DPA & Total Fluence & DPA \\
\hline $3.60 \mathrm{E}-04$ & 0.01 & $5.15 \mathrm{E}+19$ & $6.43 \mathrm{E}-06$ & $6.11 \mathrm{E}+19$ & 7.64E-06 & $6.98 \mathrm{E}+19$ & 8.73E-06 & $7.57 \mathrm{E}+19$ & $9.46 \mathrm{E}-06$ \\
\hline 4.50E-04 & 0.007 & $4.91 \mathrm{E}+19$ & $4.30 \mathrm{E}-06$ & $5.34 \mathrm{E}+19$ & 4.67E-06 & $6.31 \mathrm{E}+19$ & $5.52 \mathrm{E}-06$ & $6.85 \mathrm{E}+19$ & 5.99E-06 \\
\hline $5.75 \mathrm{E}-04$ & 0.005 & $5.40 \mathrm{E}+19$ & $3.38 \mathrm{E}-06$ & $6.14 \mathrm{E}+19$ & $3.84 \mathrm{E}-06$ & $7.03 E+19$ & 4.39E-06 & $8.03 E+19$ & $5.02 \mathrm{E}-06$ \\
\hline $7.60 \mathrm{E}-04$ & 0.129 & $6.20 \mathrm{E}+19$ & 9.99E-05 & $6.80 \mathrm{E}+19$ & $1.10 \mathrm{E}-04$ & $7.92 \mathrm{E}+19$ & $1.28 \mathrm{E}-04$ & $9.11 \mathrm{E}+19$ & 1.47E-04 \\
\hline $9.60 \mathrm{E}-04$ & 0.308 & $5.33 \mathrm{E}+19$ & $2.05 \mathrm{E}-04$ & $5.85 \mathrm{E}+19$ & $2.25 \mathrm{E}-04$ & $6.67 \mathrm{E}+19$ & 2.57E-04 & $7.66 \mathrm{E}+19$ & $2.95 \mathrm{E}-04$ \\
\hline $1.28 \mathrm{E}-03$ & 0.672 & $6.07 \mathrm{E}+19$ & $5.10 \mathrm{E}-04$ & 7.17E+19 & $6.03 \mathrm{E}-04$ & $8.23 E+19$ & $6.91 \mathrm{E}-04$ & $9.21 \mathrm{E}+19$ & 7.74E-04 \\
\hline $1.60 \mathrm{E}-03$ & 0.477 & $4.99 \mathrm{E}+19$ & 2.97E-04 & $5.54 \mathrm{E}+19$ & $3.31 \mathrm{E}-04$ & $6.43 \mathrm{E}+19$ & 3.83E-04 & $7.02 E+19$ & 4.19E-04 \\
\hline $2.00 \mathrm{E}-03$ & 0.511 & $4.89 \mathrm{E}+19$ & $3.12 \mathrm{E}-04$ & $5.85 \mathrm{E}+19$ & $3.74 \mathrm{E}-04$ & $6.13 E+19$ & $3.91 \mathrm{E}-04$ & $7.04 \mathrm{E}+19$ & 4.50E-04 \\
\hline $2.70 \mathrm{E}-03$ & 0.568 & $6.48 \mathrm{E}+19$ & $4.60 \mathrm{E}-04$ & $7.81 \mathrm{E}+19$ & $5.55 \mathrm{E}-04$ & $8.20 \mathrm{E}+19$ & $5.82 \mathrm{E}-04$ & $9.66 \mathrm{E}+19$ & $6.86 \mathrm{E}-04$ \\
\hline $3.40 \mathrm{E}-03$ & 0.632 & $5.01 \mathrm{E}+19$ & $3.96 \mathrm{E}-04$ & $6.17 \mathrm{E}+19$ & 4.87E-04 & $6.64 \mathrm{E}+19$ & $5.25 \mathrm{E}-04$ & $7.82 \mathrm{E}+19$ & $6.18 \mathrm{E}-04$ \\
\hline 4.50E-03 & 0.822 & $6.21 \mathrm{E}+19$ & $6.38 \mathrm{E}-04$ & $7.13 \mathrm{E}+19$ & 7.32E-04 & $8.35 E+19$ & $8.58 \mathrm{E}-04$ & $9.44 \mathrm{E}+19$ & $9.70 \mathrm{E}-04$ \\
\hline $5.50 \mathrm{E}-03$ & 0.826 & 4.76E+19 & $4.91 \mathrm{E}-04$ & $5.22 \mathrm{E}+19$ & $5.39 \mathrm{E}-04$ & $6.11 E+19$ & $6.31 \mathrm{E}-04$ & $7.20 \mathrm{E}+19$ & 7.43E-04 \\
\hline 7.20E-03 & 1.671 & $6.09 \mathrm{E}+19$ & $1.27 \mathrm{E}-03$ & $7.29 \mathrm{E}+19$ & $1.52 \mathrm{E}-03$ & $8.79 \mathrm{E}+19$ & $1.84 \mathrm{E}-03$ & $9.39 \mathrm{E}+19$ & $1.96 \mathrm{E}-03$ \\
\hline $9.20 \mathrm{E}-03$ & 3.709 & $5.15 \mathrm{E}+19$ & $2.39 \mathrm{E}-03$ & $6.02 \mathrm{E}+19$ & $2.79 \mathrm{E}-03$ & $6.45 \mathrm{E}+19$ & $2.99 \mathrm{E}-03$ & $7.48 \mathrm{E}+19$ & 3.47E-03 \\
\hline $1.20 \mathrm{E}-02$ & 1.491 & $6.40 \mathrm{E}+19$ & $1.19 \mathrm{E}-03$ & $6.74 \mathrm{E}+19$ & $1.26 \mathrm{E}-03$ & $8.01 E+19$ & $1.49 \mathrm{E}-03$ & $9.03 E+19$ & $1.68 \mathrm{E}-03$ \\
\hline $1.50 \mathrm{E}-02$ & 1.156 & $5.36 \mathrm{E}+19$ & 7.74E-04 & $5.87 \mathrm{E}+19$ & $8.48 \mathrm{E}-04$ & $7.08 \mathrm{E}+19$ & $1.02 \mathrm{E}-03$ & $7.83 \mathrm{E}+19$ & 1.13E-03 \\
\hline $1.90 \mathrm{E}-02$ & 0.973 & $5.65 \mathrm{E}+19$ & $6.87 \mathrm{E}-04$ & $6.35 \mathrm{E}+19$ & 7.72E-04 & $7.58 \mathrm{E}+19$ & $9.22 \mathrm{E}-04$ & $8.26 \mathrm{E}+19$ & $1.01 \mathrm{E}-03$ \\
\hline $2.55 \mathrm{E}-02$ & 0.555 & $8.39 \mathrm{E}+19$ & $5.82 \mathrm{E}-04$ & $9.29 \mathrm{E}+19$ & $6.44 \mathrm{E}-04$ & $1.08 \mathrm{E}+20$ & $7.48 \mathrm{E}-04$ & $1.21 \mathrm{E}+20$ & $8.38 \mathrm{E}-04$ \\
\hline $3.20 \mathrm{E}-02$ & 26.762 & $7.03 E+19$ & $2.35 \mathrm{E}-02$ & $8.38 \mathrm{E}+19$ & $2.80 \mathrm{E}-02$ & $9.42 \mathrm{E}+19$ & $3.15 \mathrm{E}-02$ & $1.07 \mathrm{E}+20$ & $3.59 \mathrm{E}-02$ \\
\hline $4.00 \mathrm{E}-02$ & 8.161 & $3.53 \mathrm{E}+19$ & $3.60 \mathrm{E}-03$ & $4.18 \mathrm{E}+19$ & 4.27E-03 & $4.64 \mathrm{E}+19$ & $4.73 \mathrm{E}-03$ & $5.23 \mathrm{E}+19$ & $5.34 \mathrm{E}-03$ \\
\hline $5.25 \mathrm{E}-02$ & 6.515 & $7.06 \mathrm{E}+19$ & $5.75 \mathrm{E}-03$ & $8.36 \mathrm{E}+19$ & 6.80E-03 & $9.04 \mathrm{E}+19$ & $7.36 \mathrm{E}-03$ & $1.05 \mathrm{E}+20$ & $8.58 \mathrm{E}-03$ \\
\hline $6.60 \mathrm{E}-02$ & 6.576 & $7.28 \mathrm{E}+19$ & $5.98 \mathrm{E}-03$ & $8.45 \mathrm{E}+19$ & $6.95 \mathrm{E}-03$ & $9.05 \mathrm{E}+19$ & $7.44 \mathrm{E}-03$ & $1.05 \mathrm{E}+20$ & 8.64E-03 \\
\hline $8.80 \mathrm{E}-02$ & 13.137 & $1.08 \mathrm{E}+20$ & $1.77 \mathrm{E}-02$ & $1.17 \mathrm{E}+20$ & $1.92 \mathrm{E}-02$ & $1.36 \mathrm{E}+20$ & $2.23 \mathrm{E}-02$ & $1.53 \mathrm{E}+20$ & $2.52 \mathrm{E}-02$ \\
\hline $1.10 \mathrm{E}-01$ & 11.84 & $6.61 \mathrm{E}+19$ & $9.78 \mathrm{E}-03$ & $7.54 \mathrm{E}+19$ & $1.12 \mathrm{E}-02$ & $8.53 E+19$ & $1.26 \mathrm{E}-02$ & $9.47 E+19$ & $1.40 \mathrm{E}-02$ \\
\hline $1.35 \mathrm{E}-01$ & 11.27 & $8.93 \mathrm{E}+19$ & $1.26 \mathrm{E}-02$ & $1.08 \mathrm{E}+20$ & $1.52 \mathrm{E}-02$ & $1.24 \mathrm{E}+20$ & $1.75 \mathrm{E}-02$ & $1.41 \mathrm{E}+20$ & $1.98 \mathrm{E}-02$ \\
\hline $1.60 \mathrm{E}-01$ & 21.53 & $5.24 \mathrm{E}+19$ & $1.41 \mathrm{E}-02$ & $6.44 \mathrm{E}+19$ & $1.73 \mathrm{E}-02$ & $7.33 E+19$ & $1.97 \mathrm{E}-02$ & $8.03 E+19$ & $2.16 \mathrm{E}-02$ \\
\hline $1.90 \mathrm{E}-01$ & 15.85 & $7.80 \mathrm{E}+19$ & $1.54 \mathrm{E}-02$ & $9.15 \mathrm{E}+19$ & $1.81 \mathrm{E}-02$ & $1.02 \mathrm{E}+20$ & $2.01 \mathrm{E}-02$ & $1.15 \mathrm{E}+20$ & $2.29 \mathrm{E}-02$ \\
\hline $2.20 \mathrm{E}-01$ & 22.01 & $6.13 \mathrm{E}+19$ & $1.69 \mathrm{E}-02$ & $7.27 \mathrm{E}+19$ & $2.00 \mathrm{E}-02$ & $8.20 \mathrm{E}+19$ & $2.26 \mathrm{E}-02$ & $9.37 E+19$ & $2.58 \mathrm{E}-02$ \\
\hline $2.55 \mathrm{E}-01$ & 18.27 & $7.46 \mathrm{E}+19$ & $1.70 \mathrm{E}-02$ & $8.35 \mathrm{E}+19$ & $1.91 \mathrm{E}-02$ & $9.59 \mathrm{E}+19$ & $2.19 \mathrm{E}-02$ & $1.10 \mathrm{E}+20$ & $2.51 \mathrm{E}-02$ \\
\hline $2.90 \mathrm{E}-01$ & 17.37 & $8.26 \mathrm{E}+19$ & $1.79 \mathrm{E}-02$ & $9.37 \mathrm{E}+19$ & $2.03 \mathrm{E}-02$ & $1.08 \mathrm{E}+20$ & $2.34 \mathrm{E}-02$ & $1.17 \mathrm{E}+20$ & $2.55 \mathrm{E}-02$ \\
\hline
\end{tabular}




\begin{tabular}{|c|c|c|c|c|c|c|c|c|c|}
\hline \multirow[b]{2}{*}{ Upper Energy(MeV) } & \multirow{2}{*}{$\begin{array}{l}\text { DPA xsec } \\
\text { keV-barn }\end{array}$} & \multicolumn{2}{|c|}{$10-4$} & \multicolumn{2}{|c|}{$10-3$} & \multicolumn{2}{|c|}{$10-2$} & \multicolumn{2}{|c|}{$10-1$} \\
\hline & & Total Fluence & DPA & Total Fluence & DPA & Total Fluence & DPA & Total Fluence & DPA \\
\hline $3.20 \mathrm{E}-01$ & 13.51 & $5.95 \mathrm{E}+19$ & $1.00 \mathrm{E}-02$ & $6.67 \mathrm{E}+19$ & $1.13 \mathrm{E}-02$ & $7.45 \mathrm{E}+19$ & $1.26 \mathrm{E}-02$ & $8.13 E+19$ & $1.37 \mathrm{E}-02$ \\
\hline $3.60 \mathrm{E}-01$ & 25.14 & $8.22 \mathrm{E}+19$ & $2.58 \mathrm{E}-02$ & $9.37 \mathrm{E}+19$ & 2.95E-02 & $1.07 \mathrm{E}+20$ & $3.35 \mathrm{E}-02$ & 1.17E+20 & $3.68 \mathrm{E}-02$ \\
\hline $4.00 \mathrm{E}-01$ & 46.52 & $6.99 \mathrm{E}+19$ & $4.06 \mathrm{E}-02$ & $7.67 \mathrm{E}+19$ & $4.46 \mathrm{E}-02$ & $9.00 \mathrm{E}+19$ & $5.24 \mathrm{E}-02$ & $9.62 E+19$ & $5.59 \mathrm{E}-02$ \\
\hline 4.50E-01 & 43.04 & $6.31 E+19$ & $3.40 \mathrm{E}-02$ & $7.12 \mathrm{E}+19$ & 3.83E-02 & $8.37 \mathrm{E}+19$ & $4.50 \mathrm{E}-02$ & $9.38 \mathrm{E}+19$ & $5.05 \mathrm{E}-02$ \\
\hline $5.00 \mathrm{E}-01$ & 36.4 & $7.62 \mathrm{E}+19$ & $3.47 \mathrm{E}-02$ & $8.46 \mathrm{E}+19$ & $3.85 \mathrm{E}-02$ & $9.40 \mathrm{E}+19$ & $4.28 \mathrm{E}-02$ & $1.07 \mathrm{E}+20$ & 4.87E-02 \\
\hline $5.50 \mathrm{E}-01$ & 33.22 & $7.31 \mathrm{E}+19$ & $3.04 \mathrm{E}-02$ & $8.28 \mathrm{E}+19$ & $3.44 \mathrm{E}-02$ & $9.66 \mathrm{E}+19$ & 4.01E-02 & $1.01 \mathrm{E}+20$ & $4.18 \mathrm{E}-02$ \\
\hline $6.00 \mathrm{E}-01$ & 34.18 & $6.94 \mathrm{E}+19$ & $2.96 \mathrm{E}-02$ & $8.14 \mathrm{E}+19$ & $3.48 \mathrm{E}-02$ & $9.07 E+19$ & 3.87E-02 & $1.00 \mathrm{E}+20$ & $4.29 \mathrm{E}-02$ \\
\hline $6.60 \mathrm{E}-01$ & 19.64 & $8.58 \mathrm{E}+19$ & $2.11 \mathrm{E}-02$ & $1.03 E+20$ & $2.53 \mathrm{E}-02$ & 1.10E+20 & $2.70 \mathrm{E}-02$ & $1.26 \mathrm{E}+20$ & $3.10 \mathrm{E}-02$ \\
\hline $7.20 \mathrm{E}-01$ & 48.89 & $8.92 E+19$ & $5.45 \mathrm{E}-02$ & $1.01 \mathrm{E}+20$ & $6.20 \mathrm{E}-02$ & $1.14 \mathrm{E}+20$ & $6.98 \mathrm{E}-02$ & $1.29 \mathrm{E}+20$ & $7.91 \mathrm{E}-02$ \\
\hline $7.80 \mathrm{E}-01$ & 74.22 & $8.08 \mathrm{E}+19$ & $7.50 \mathrm{E}-02$ & $9.52 \mathrm{E}+19$ & $8.84 \mathrm{E}-02$ & $1.07 \mathrm{E}+20$ & $9.90 \mathrm{E}-02$ & $1.19 \mathrm{E}+20$ & $1.11 \mathrm{E}-01$ \\
\hline $8.40 \mathrm{E}-01$ & 44.02 & $6.72 \mathrm{E}+19$ & $3.70 \mathrm{E}-02$ & $7.48 \mathrm{E}+19$ & 4.12E-02 & 8.24E+19 & $4.53 \mathrm{E}-02$ & $9.31 E+19$ & $5.12 \mathrm{E}-02$ \\
\hline $9.20 \mathrm{E}-01$ & 40.97 & $7.86 \mathrm{E}+19$ & $4.02 \mathrm{E}-02$ & $8.98 \mathrm{E}+19$ & 4.60E-02 & $1.05 \mathrm{E}+20$ & $5.40 \mathrm{E}-02$ & $1.13 \mathrm{E}+20$ & $5.78 \mathrm{E}-02$ \\
\hline $1.00 \mathrm{E}+00$ & 50.95 & $7.22 \mathrm{E}+19$ & $4.60 \mathrm{E}-02$ & $8.63 E+19$ & $5.50 \mathrm{E}-02$ & $9.61 \mathrm{E}+19$ & $6.12 \mathrm{E}-02$ & $1.02 \mathrm{E}+20$ & $6.49 \mathrm{E}-02$ \\
\hline $1.20 \mathrm{E}+00$ & 50.09 & $1.73 \mathrm{E}+20$ & $1.08 \mathrm{E}-01$ & $1.98 \mathrm{E}+20$ & $1.24 \mathrm{E}-01$ & $2.19 \mathrm{E}+20$ & $1.37 \mathrm{E}-01$ & $2.43 \mathrm{E}+20$ & $1.52 \mathrm{E}-01$ \\
\hline $1.40 \mathrm{E}+00$ & 64.54 & $1.61 \mathrm{E}+20$ & $1.30 \mathrm{E}-01$ & $1.86 \mathrm{E}+20$ & $1.50 \mathrm{E}-01$ & $1.98 \mathrm{E}+20$ & $1.60 \mathrm{E}-01$ & $2.18 \mathrm{E}+20$ & $1.76 \mathrm{E}-01$ \\
\hline $1.60 \mathrm{E}+00$ & 73.58 & $1.31 \mathrm{E}+20$ & $1.20 \mathrm{E}-01$ & $1.47 \mathrm{E}+20$ & $1.36 \mathrm{E}-01$ & $1.69 \mathrm{E}+20$ & $1.55 \mathrm{E}-01$ & $1.84 \mathrm{E}+20$ & $1.69 \mathrm{E}-01$ \\
\hline $1.80 \mathrm{E}+00$ & 76.46 & $1.13 \mathrm{E}+20$ & $1.08 \mathrm{E}-01$ & $1.31 \mathrm{E}+20$ & $1.25 \mathrm{E}-01$ & $1.49 \mathrm{E}+20$ & $1.43 \mathrm{E}-01$ & $1.63 \mathrm{E}+20$ & $1.55 \mathrm{E}-01$ \\
\hline $2.00 \mathrm{E}+00$ & 95.15 & $1.06 \mathrm{E}+20$ & $1.26 \mathrm{E}-01$ & $1.14 \mathrm{E}+20$ & $1.36 \mathrm{E}-01$ & $1.28 \mathrm{E}+20$ & $1.52 \mathrm{E}-01$ & $1.44 \mathrm{E}+20$ & $1.71 \mathrm{E}-01$ \\
\hline $2.30 \mathrm{E}+00$ & 93.75 & $1.26 \mathrm{E}+20$ & $1.47 \mathrm{E}-01$ & $1.45 \mathrm{E}+20$ & $1.70 \mathrm{E}-01$ & $1.63 \mathrm{E}+20$ & $1.91 \mathrm{E}-01$ & $1.79 \mathrm{E}+20$ & $2.10 \mathrm{E}-01$ \\
\hline $2.60 \mathrm{E}+00$ & 112.05 & $1.16 \mathrm{E}+20$ & $1.63 \mathrm{E}-01$ & $1.36 \mathrm{E}+20$ & $1.91 \mathrm{E}-01$ & $1.49 \mathrm{E}+20$ & $2.09 \mathrm{E}-01$ & $1.66 \mathrm{E}+20$ & $2.33 \mathrm{E}-01$ \\
\hline $2.90 \mathrm{E}+00$ & 123.55 & $9.03 E+19$ & $1.39 \mathrm{E}-01$ & $9.84 \mathrm{E}+19$ & $1.52 \mathrm{E}-01$ & $1.09 \mathrm{E}+20$ & $1.69 \mathrm{E}-01$ & $1.24 \mathrm{E}+20$ & $1.92 \mathrm{E}-01$ \\
\hline $3.30 \mathrm{E}+00$ & 133.45 & $8.74 \mathrm{E}+19$ & $1.46 \mathrm{E}-01$ & $9.96 \mathrm{E}+19$ & $1.66 \mathrm{E}-01$ & $1.11 \mathrm{E}+20$ & $1.86 \mathrm{E}-01$ & $1.24 \mathrm{E}+20$ & $2.07 \mathrm{E}-01$ \\
\hline $3.70 \mathrm{E}+00$ & 135.25 & $5.98 \mathrm{E}+19$ & $1.01 \mathrm{E}-01$ & $7.07 \mathrm{E}+19$ & $1.19 \mathrm{E}-01$ & $7.61 \mathrm{E}+19$ & $1.29 \mathrm{E}-01$ & $8.36 \mathrm{E}+19$ & $1.41 \mathrm{E}-01$ \\
\hline 4.10E+00 & 149.55 & $4.38 \mathrm{E}+19$ & $8.19 \mathrm{E}-02$ & $5.36 \mathrm{E}+19$ & $1.00 \mathrm{E}-01$ & $5.86 \mathrm{E}+19$ & $1.10 \mathrm{E}-01$ & $6.74 \mathrm{E}+19$ & $1.26 \mathrm{E}-01$ \\
\hline $4.50 \mathrm{E}+00$ & 158.25 & $4.11 \mathrm{E}+19$ & $8.13 \mathrm{E}-02$ & $4.08 \mathrm{E}+19$ & 8.06E-02 & $4.76 \mathrm{E}+19$ & $9.42 \mathrm{E}-02$ & $4.99 \mathrm{E}+19$ & $9.88 \mathrm{E}-02$ \\
\hline $5.00 \mathrm{E}+00$ & 168.55 & $3.25 \mathrm{E}+19$ & $6.85 \mathrm{E}-02$ & $3.83 E+19$ & 8.07E-02 & $4.28 \mathrm{E}+19$ & $9.01 \mathrm{E}-02$ & $4.74 \mathrm{E}+19$ & $9.99 \mathrm{E}-02$ \\
\hline $5.50 \mathrm{E}+00$ & 176.46 & $2.26 \mathrm{E}+19$ & 4.99E-02 & $2.64 \mathrm{E}+19$ & $5.83 \mathrm{E}-02$ & $3.02 \mathrm{E}+19$ & $6.67 \mathrm{E}-02$ & $3.33 E+19$ & $7.34 \mathrm{E}-02$ \\
\hline $6.00 \mathrm{E}+00$ & 183.06 & $1.54 \mathrm{E}+19$ & $3.54 \mathrm{E}-02$ & $1.73 \mathrm{E}+19$ & $3.97 \mathrm{E}-02$ & $2.15 \mathrm{E}+19$ & 4.92E-02 & $2.24 \mathrm{E}+19$ & $5.12 \mathrm{E}-02$ \\
\hline $6.70 \mathrm{E}+00$ & 189.26 & $1.50 \mathrm{E}+19$ & $3.55 \mathrm{E}-02$ & $1.69 \mathrm{E}+19$ & 4.01E-02 & $1.97 \mathrm{E}+19$ & 4.66E-02 & $2.03 E+19$ & $4.80 \mathrm{E}-02$ \\
\hline $7.40 \mathrm{E}+00$ & 196.67 & $8.28 \mathrm{E}+18$ & $2.03 \mathrm{E}-02$ & $1.04 \mathrm{E}+19$ & $2.56 \mathrm{E}-02$ & $1.06 \mathrm{E}+19$ & $2.61 \mathrm{E}-02$ & $1.11 \mathrm{E}+19$ & $2.73 \mathrm{E}-02$ \\
\hline
\end{tabular}




\begin{tabular}{|c|c|c|c|c|c|c|c|c|c|}
\hline \multirow[b]{2}{*}{ Upper Energy(MeV) } & \multirow{2}{*}{$\begin{array}{l}\text { DPA xsec } \\
\text { keV-barn }\end{array}$} & \multicolumn{2}{|c|}{$10-4$} & \multicolumn{2}{|c|}{$10-3$} & \multicolumn{2}{|c|}{$10-2$} & \multicolumn{2}{|c|}{$10-1$} \\
\hline & & Total Fluence & DPA & Total Fluence & DPA & Total Fluence & DPA & Total Fluence & DPA \\
\hline $8.20 \mathrm{E}+00$ & 203.37 & $4.72 \mathrm{E}+18$ & $1.20 \mathrm{E}-02$ & $6.36 \mathrm{E}+18$ & $1.62 \mathrm{E}-02$ & $6.29 \mathrm{E}+18$ & $1.60 \mathrm{E}-02$ & $7.37 \mathrm{E}+18$ & 1.87E-02 \\
\hline $9.00 \mathrm{E}+00$ & 214.58 & $3.14 \mathrm{E}+18$ & $8.41 \mathrm{E}-03$ & $2.18 \mathrm{E}+18$ & $5.84 \mathrm{E}-03$ & $4.39 \mathrm{E}+18$ & $1.18 \mathrm{E}-02$ & $3.89 \mathrm{E}+18$ & $1.04 \mathrm{E}-02$ \\
\hline $1.00 \mathrm{E}+01$ & 225.69 & $1.68 \mathrm{E}+18$ & 4.73E-03 & $2.20 \mathrm{E}+18$ & $6.21 \mathrm{E}-03$ & $2.05 E+18$ & 5.77E-03 & $2.67 E+18$ & 7.54E-03 \\
\hline $1.10 \mathrm{E}+01$ & 237.6 & $7.76 \mathrm{E}+17$ & $2.31 \mathrm{E}-03$ & $6.98 \mathrm{E}+17$ & 2.07E-03 & $9.90 \mathrm{E}+17$ & $2.94 \mathrm{E}-03$ & $1.15 \mathrm{E}+18$ & $3.42 \mathrm{E}-03$ \\
\hline $1.20 \mathrm{E}+01$ & 247.41 & $4.01 \mathrm{E}+17$ & $1.24 \mathrm{E}-03$ & $3.09 E+17$ & $9.56 \mathrm{E}-04$ & $4.51 E+17$ & $1.40 \mathrm{E}-03$ & $2.88 \mathrm{E}+17$ & 8.90E-04 \\
\hline $1.30 \mathrm{E}+01$ & 258.52 & $3.85 \mathrm{E}+17$ & $1.24 \mathrm{E}-03$ & $3.63 \mathrm{E}+17$ & 1.17E-03 & $3.50 \mathrm{E}+17$ & $1.13 \mathrm{E}-03$ & $3.44 \mathrm{E}+17$ & $1.11 \mathrm{E}-03$ \\
\hline $1.40 \mathrm{E}+01$ & 271.35 & $8.21 \mathrm{E}+16$ & $2.78 \mathrm{E}-04$ & $8.72 \mathrm{E}+16$ & $2.96 \mathrm{E}-04$ & $1.42 \mathrm{E}+16$ & $4.82 \mathrm{E}-05$ & $1.37 \mathrm{E}+17$ & 4.64E-04 \\
\hline $1.50 \mathrm{E}+01$ & 290.27 & $6.69 \mathrm{E}+16$ & $2.43 \mathrm{E}-04$ & $4.15 E+16$ & $1.50 \mathrm{E}-04$ & $8.84 \mathrm{E}+16$ & $3.21 \mathrm{E}-04$ & $2.20 E+16$ & 7.97E-05 \\
\hline $1.60 \mathrm{E}+01$ & 293.2 & $6.47 \mathrm{E}+15$ & 2.37E-05 & $8.17 \mathrm{E}+16$ & 2.99E-04 & $0.00 \mathrm{E}+00$ & $0.00 \mathrm{E}+00$ & $0.00 \mathrm{E}+00$ & $0.00 \mathrm{E}+00$ \\
\hline $1.70 \mathrm{E}+01$ & 292.73 & $0.00 \mathrm{E}+00$ & $0.00 \mathrm{E}+00$ & $0.00 \mathrm{E}+00$ & $0.00 \mathrm{E}+00$ & $0.00 \mathrm{E}+00$ & $0.00 \mathrm{E}+00$ & $2.73 E+16$ & 9.99E-05 \\
\hline $1.80 \mathrm{E}+01$ & 297.65 & $0.00 \mathrm{E}+00$ & $0.00 \mathrm{E}+00$ & $0.00 \mathrm{E}+00$ & $0.00 \mathrm{E}+00$ & $1.61 \mathrm{E}+16$ & $5.98 \mathrm{E}-05$ & $0.00 \mathrm{E}+00$ & $0.00 \mathrm{E}+00$ \\
\hline $1.90 \mathrm{E}+01$ & 307.26 & $0.00 \mathrm{E}+00$ & $0.00 \mathrm{E}+00$ & $0.00 \mathrm{E}+00$ & $0.00 \mathrm{E}+00$ & $0.00 \mathrm{E}+00$ & $0.00 \mathrm{E}+00$ & $0.00 \mathrm{E}+00$ & $0.00 \mathrm{E}+00$ \\
\hline $2.00 \mathrm{E}+01$ & 316.36 & $0.00 E+00$ & $0.00 E+00$ & $0.00 E+00$ & $0.00 E+00$ & $0.00 E+00$ & $0.00 \mathrm{E}+00$ & $0.00 E+00$ & $0.00 \mathrm{E}+00$ \\
\hline total & & & $2.36 E+00$ & & $2.69 E+00$ & & $3.02 E+00$ & & $3.33 E+00$ \\
\hline
\end{tabular}


Table 12. Calculated fluence and DPA for capsule 9 of the UCSB experiment.

\begin{tabular}{|c|c|c|c|c|c|c|c|c|c|}
\hline \multirow{2}{*}{$\begin{array}{c}\text { Upper } \\
\text { Energy(MeV) }\end{array}$} & \multirow{2}{*}{$\begin{array}{l}\text { DPA xsec } \\
\text { keV-barn }\end{array}$} & \multicolumn{2}{|c|}{$9-4$} & \multicolumn{2}{|c|}{$9-3$} & \multicolumn{2}{|c|}{$9-2$} & \multicolumn{2}{|c|}{$9-1$} \\
\hline & & Total Fluence & DPA & Total Fluence & DPA & Total Fluence & DPA & Total Fluence & DPA \\
\hline $1.00 \mathrm{E}-10$ & 0 & 4.70E+15 & & $6.61 \mathrm{E}+14$ & & $0.00 E+00$ & & $1.43 E+15$ & \\
\hline $1.00 \mathrm{E}-09$ & 7.73 & $5.08 \mathrm{E}+17$ & 4.91E-05 & $5.45 \mathrm{E}+17$ & $5.27 \mathrm{E}-05$ & $5.46 \mathrm{E}+17$ & $5.28 \mathrm{E}-05$ & $8.01 \mathrm{E}+17$ & 7.74E-05 \\
\hline $1.00 \mathrm{E}-08$ & 2.444 & $7.93 \mathrm{E}+19$ & $2.42 \mathrm{E}-03$ & $8.58 \mathrm{E}+19$ & 2.62E-03 & $9.33 E+19$ & $2.85 \mathrm{E}-03$ & $1.03 E+20$ & $3.13 \mathrm{E}-03$ \\
\hline $2.30 \mathrm{E}-08$ & 1.278 & $2.87 E+20$ & $4.58 \mathrm{E}-03$ & $3.03 E+20$ & 4.84E-03 & $3.38 \mathrm{E}+20$ & $5.40 \mathrm{E}-03$ & $3.67 E+20$ & $5.86 \mathrm{E}-03$ \\
\hline $5.00 \mathrm{E}-08$ & 0.857 & $7.10 \mathrm{E}+20$ & 7.61E-03 & $7.66 \mathrm{E}+20$ & $8.21 \mathrm{E}-03$ & $8.50 \mathrm{E}+20$ & $9.10 \mathrm{E}-03$ & $9.14 \mathrm{E}+20$ & $9.80 \mathrm{E}-03$ \\
\hline $7.60 \mathrm{E}-08$ & 0.644 & $5.04 \mathrm{E}+20$ & 4.05E-03 & $5.45 E+20$ & 4.38E-03 & $6.05 E+20$ & 4.87E-03 & $6.50 \mathrm{E}+20$ & $5.23 \mathrm{E}-03$ \\
\hline $1.15 \mathrm{E}-07$ & 0.523 & $4.35 E+20$ & $2.84 \mathrm{E}-03$ & $4.75 \mathrm{E}+20$ & $3.11 \mathrm{E}-03$ & $5.20 \mathrm{E}+20$ & $3.40 \mathrm{E}-03$ & $5.60 \mathrm{E}+20$ & 3.66E-03 \\
\hline $1.70 \mathrm{E}-07$ & 0.428 & $2.78 \mathrm{E}+20$ & 1.49E-03 & $3.03 E+20$ & $1.62 \mathrm{E}-03$ & $3.27 E+20$ & $1.75 \mathrm{E}-03$ & $3.57 E+20$ & $1.91 \mathrm{E}-03$ \\
\hline $2.55 \mathrm{E}-07$ & 0.351 & $1.93 E+20$ & 8.49E-04 & $2.09 \mathrm{E}+20$ & $9.18 \mathrm{E}-04$ & $2.32 \mathrm{E}+20$ & $1.02 \mathrm{E}-03$ & $2.49 \mathrm{E}+20$ & $1.09 \mathrm{E}-03$ \\
\hline $3.80 \mathrm{E}-07$ & 0.287 & $1.64 \mathrm{E}+20$ & $5.88 \mathrm{E}-04$ & $1.78 \mathrm{E}+20$ & $6.39 \mathrm{E}-04$ & $1.95 \mathrm{E}+20$ & $6.99 \mathrm{E}-04$ & $2.04 \mathrm{E}+20$ & 7.31E-04 \\
\hline $5.50 \mathrm{E}-07$ & 0.237 & $1.45 \mathrm{E}+20$ & 4.30E-04 & $1.60 \mathrm{E}+20$ & $4.75 \mathrm{E}-04$ & $1.76 \mathrm{E}+20$ & $5.21 \mathrm{E}-04$ & $1.90 \mathrm{E}+20$ & 5.61E-04 \\
\hline $8.40 \mathrm{E}-07$ & 0.194 & $1.66 \mathrm{E}+20$ & 4.03E-04 & $1.78 \mathrm{E}+20$ & 4.32E-04 & $1.94 \mathrm{E}+20$ & 4.70E-04 & $2.05 E+20$ & 4.97E-04 \\
\hline $1.28 \mathrm{E}-06$ & 0.157 & $1.57 \mathrm{E}+20$ & $3.08 \mathrm{E}-04$ & $1.67 \mathrm{E}+20$ & 3.29E-04 & $1.93 \mathrm{E}+20$ & 3.80E-04 & $1.97 \mathrm{E}+20$ & 3.87E-04 \\
\hline $1.90 \mathrm{E}-06$ & 0.128 & $1.43 E+20$ & $2.29 \mathrm{E}-04$ & $1.62 \mathrm{E}+20$ & 2.59E-04 & $1.76 \mathrm{E}+20$ & $2.82 \mathrm{E}-04$ & $1.81 \mathrm{E}+20$ & $2.89 \mathrm{E}-04$ \\
\hline $2.80 \mathrm{E}-06$ & 0.105 & $1.42 \mathrm{E}+20$ & $1.87 \mathrm{E}-04$ & $1.56 \mathrm{E}+20$ & $2.04 \mathrm{E}-04$ & $1.70 \mathrm{E}+20$ & $2.23 \mathrm{E}-04$ & $1.81 \mathrm{E}+20$ & $2.38 \mathrm{E}-04$ \\
\hline 4.25E-06 & 0.086 & $1.55 \mathrm{E}+20$ & $1.67 \mathrm{E}-04$ & $1.50 \mathrm{E}+20$ & $1.61 \mathrm{E}-04$ & $1.79 \mathrm{E}+20$ & $1.92 \mathrm{E}-04$ & $1.88 \mathrm{E}+20$ & 2.03E-04 \\
\hline $6.30 \mathrm{E}-06$ & 0.07 & $1.45 \mathrm{E}+20$ & $1.27 \mathrm{E}-04$ & $1.53 \mathrm{E}+20$ & $1.34 \mathrm{E}-04$ & $1.64 \mathrm{E}+20$ & $1.43 \mathrm{E}-04$ & $1.75 \mathrm{E}+20$ & $1.53 \mathrm{E}-04$ \\
\hline $9.20 \mathrm{E}-06$ & 0.058 & $1.42 \mathrm{E}+20$ & $1.03 \mathrm{E}-04$ & $1.46 \mathrm{E}+20$ & $1.06 \mathrm{E}-04$ & $1.59 \mathrm{E}+20$ & $1.15 \mathrm{E}-04$ & $1.73 \mathrm{E}+20$ & $1.26 \mathrm{E}-04$ \\
\hline $1.35 \mathrm{E}-05$ & 0.048 & $1.43 \mathrm{E}+20$ & 8.57E-05 & $1.58 \mathrm{E}+20$ & $9.47 \mathrm{E}-05$ & $1.66 \mathrm{E}+20$ & $9.95 \mathrm{E}-05$ & $1.81 \mathrm{E}+20$ & $1.08 \mathrm{E}-04$ \\
\hline $2.10 \mathrm{E}-05$ & 0.038 & $1.65 \mathrm{E}+20$ & $7.85 \mathrm{E}-05$ & $1.53 \mathrm{E}+20$ & 7.26E-05 & $1.88 \mathrm{E}+20$ & $8.94 \mathrm{E}-05$ & $2.01 \mathrm{E}+20$ & 9.57E-05 \\
\hline $3.00 \mathrm{E}-05$ & 0.031 & $1.39 \mathrm{E}+20$ & $5.40 \mathrm{E}-05$ & $1.32 \mathrm{E}+20$ & $5.12 \mathrm{E}-05$ & $1.58 \mathrm{E}+20$ & $6.11 \mathrm{E}-05$ & $1.69 \mathrm{E}+20$ & 6.56E-05 \\
\hline $4.50 \mathrm{E}-05$ & 0.026 & $1.58 \mathrm{E}+20$ & $5.13 \mathrm{E}-05$ & $1.66 \mathrm{E}+20$ & $5.40 \mathrm{E}-05$ & $1.80 \mathrm{E}+20$ & $5.85 \mathrm{E}-05$ & $1.90 \mathrm{E}+20$ & 6.19E-05 \\
\hline $6.90 \mathrm{E}-05$ & 0.021 & $1.65 \mathrm{E}+20$ & 4.34E-05 & $1.81 \mathrm{E}+20$ & 4.76E-05 & $1.93 \mathrm{E}+20$ & 5.07E-05 & $2.09 \mathrm{E}+20$ & $5.48 \mathrm{E}-05$ \\
\hline $1.00 \mathrm{E}-04$ & 0.017 & $1.47 \mathrm{E}+20$ & $3.13 \mathrm{E}-05$ & $1.58 \mathrm{E}+20$ & $3.35 \mathrm{E}-05$ & $1.68 \mathrm{E}+20$ & 3.57E-05 & $1.83 \mathrm{E}+20$ & $3.88 \mathrm{E}-05$ \\
\hline $1.35 \mathrm{E}-04$ & 0.014 & $1.12 \mathrm{E}+20$ & $1.96 \mathrm{E}-05$ & $1.27 \mathrm{E}+20$ & $2.23 \mathrm{E}-05$ & $1.42 \mathrm{E}+20$ & $2.48 \mathrm{E}-05$ & $1.45 \mathrm{E}+20$ & $2.54 \mathrm{E}-05$ \\
\hline $1.70 \mathrm{E}-04$ & 0.014 & $8.68 \mathrm{E}+19$ & $1.52 \mathrm{E}-05$ & $9.80 \mathrm{E}+19$ & $1.72 \mathrm{E}-05$ & $1.09 \mathrm{E}+20$ & $1.91 \mathrm{E}-05$ & $1.15 \mathrm{E}+20$ & 2.01E-05 \\
\hline $2.20 \mathrm{E}-04$ & 0.011 & $1.02 \mathrm{E}+20$ & $1.40 \mathrm{E}-05$ & $1.07 \mathrm{E}+20$ & 1.47E-05 & $1.22 \mathrm{E}+20$ & $1.68 \mathrm{E}-05$ & $1.28 \mathrm{E}+20$ & $1.76 \mathrm{E}-05$ \\
\hline
\end{tabular}




\begin{tabular}{|c|c|c|c|c|c|c|c|c|c|}
\hline \multirow{2}{*}{$\begin{array}{c}\text { Upper } \\
\text { Energy(MeV) }\end{array}$} & \multirow{2}{*}{$\begin{array}{l}\text { DPA xsec } \\
\text { keV-barn }\end{array}$} & \multicolumn{2}{|c|}{$9-4$} & \multicolumn{2}{|c|}{$9-3$} & \multicolumn{2}{|c|}{$9-2$} & \multicolumn{2}{|c|}{$9-1$} \\
\hline & & Total Fluence & DPA & Total Fluence & DPA & Total Fluence & DPA & Total Fluence & DPA \\
\hline $2.80 \mathrm{E}-04$ & 0.011 & $1.01 \mathrm{E}+20$ & 1.39E-05 & $1.04 \mathrm{E}+20$ & $1.43 \mathrm{E}-05$ & $1.13 E+20$ & $1.55 \mathrm{E}-05$ & $1.20 \mathrm{E}+20$ & $1.65 \mathrm{E}-05$ \\
\hline 3.60E-04 & 0.01 & $9.87 E+19$ & $1.23 \mathrm{E}-05$ & $1.07 \mathrm{E}+20$ & $1.33 \mathrm{E}-05$ & $1.13 \mathrm{E}+20$ & $1.41 \mathrm{E}-05$ & $1.24 \mathrm{E}+20$ & $1.55 \mathrm{E}-05$ \\
\hline 4.50E-04 & 0.007 & $8.73 E+19$ & 7.64E-06 & $9.65 \mathrm{E}+19$ & $8.45 \mathrm{E}-06$ & $1.01 \mathrm{E}+20$ & $8.86 \mathrm{E}-06$ & $1.10 \mathrm{E}+20$ & $9.66 \mathrm{E}-06$ \\
\hline $5.75 \mathrm{E}-04$ & 0.005 & $1.03 E+20$ & $6.41 \mathrm{E}-06$ & $1.06 \mathrm{E}+20$ & $6.60 \mathrm{E}-06$ & $1.15 \mathrm{E}+20$ & $7.20 \mathrm{E}-06$ & $1.23 \mathrm{E}+20$ & 7.67E-06 \\
\hline 7.60E-04 & 0.129 & $1.13 \mathrm{E}+20$ & $1.83 \mathrm{E}-04$ & $1.21 \mathrm{E}+20$ & $1.94 \mathrm{E}-04$ & $1.35 \mathrm{E}+20$ & 2.17E-04 & $1.37 \mathrm{E}+20$ & $2.21 \mathrm{E}-04$ \\
\hline $9.60 \mathrm{E}-04$ & 0.308 & $1.02 \mathrm{E}+20$ & 3.91E-04 & $1.04 \mathrm{E}+20$ & 4.01E-04 & $1.12 \mathrm{E}+20$ & $4.31 \mathrm{E}-04$ & $1.16 \mathrm{E}+20$ & 4.46E-04 \\
\hline $1.28 \mathrm{E}-03$ & 0.672 & $1.19 \mathrm{E}+20$ & $9.99 \mathrm{E}-04$ & $1.23 \mathrm{E}+20$ & $1.03 \mathrm{E}-03$ & $1.36 \mathrm{E}+20$ & $1.14 \mathrm{E}-03$ & $1.41 \mathrm{E}+20$ & 1.19E-03 \\
\hline $1.60 \mathrm{E}-03$ & 0.477 & $9.31 \mathrm{E}+19$ & $5.55 \mathrm{E}-04$ & $9.81 \mathrm{E}+19$ & 5.85E-04 & $1.08 \mathrm{E}+20$ & $6.44 \mathrm{E}-04$ & $1.10 \mathrm{E}+20$ & $6.58 \mathrm{E}-04$ \\
\hline 2.00E-03 & 0.511 & $8.81 \mathrm{E}+19$ & $5.63 \mathrm{E}-04$ & $9.96 \mathrm{E}+19$ & $6.36 \mathrm{E}-04$ & $1.09 \mathrm{E}+20$ & $6.95 \mathrm{E}-04$ & $1.19 \mathrm{E}+20$ & 7.61E-04 \\
\hline $2.70 \mathrm{E}-03$ & 0.568 & $1.22 \mathrm{E}+20$ & 8.66E-04 & $1.30 \mathrm{E}+20$ & $9.26 \mathrm{E}-04$ & $1.44 \mathrm{E}+20$ & $1.02 \mathrm{E}-03$ & $1.53 \mathrm{E}+20$ & $1.09 \mathrm{E}-03$ \\
\hline $3.40 \mathrm{E}-03$ & 0.632 & $9.74 \mathrm{E}+19$ & 7.70E-04 & $1.03 E+20$ & 8.13E-04 & $1.14 \mathrm{E}+20$ & $9.00 \mathrm{E}-04$ & $1.19 \mathrm{E}+20$ & $9.43 \mathrm{E}-04$ \\
\hline 4.50E-03 & 0.822 & $1.19 \mathrm{E}+20$ & $1.22 \mathrm{E}-03$ & $1.30 \mathrm{E}+20$ & $1.33 \mathrm{E}-03$ & $1.41 \mathrm{E}+20$ & $1.45 \mathrm{E}-03$ & $1.50 \mathrm{E}+20$ & $1.55 \mathrm{E}-03$ \\
\hline $5.50 \mathrm{E}-03$ & 0.826 & $8.84 \mathrm{E}+19$ & $9.12 \mathrm{E}-04$ & $9.40 \mathrm{E}+19$ & $9.70 \mathrm{E}-04$ & $1.04 \mathrm{E}+20$ & $1.08 \mathrm{E}-03$ & $1.06 \mathrm{E}+20$ & $1.10 \mathrm{E}-03$ \\
\hline 7.20E-03 & 1.671 & $1.18 \mathrm{E}+20$ & $2.46 \mathrm{E}-03$ & $1.30 \mathrm{E}+20$ & $2.71 \mathrm{E}-03$ & $1.38 \mathrm{E}+20$ & $2.88 \mathrm{E}-03$ & $1.50 \mathrm{E}+20$ & $3.13 \mathrm{E}-03$ \\
\hline $9.20 \mathrm{E}-03$ & 3.709 & $9.34 \mathrm{E}+19$ & 4.33E-03 & $1.04 \mathrm{E}+20$ & 4.83E-03 & $1.11 \mathrm{E}+20$ & $5.14 \mathrm{E}-03$ & $1.17 \mathrm{E}+20$ & $5.44 \mathrm{E}-03$ \\
\hline $1.20 \mathrm{E}-02$ & 1.491 & $1.13 \mathrm{E}+20$ & $2.11 \mathrm{E}-03$ & $1.24 \mathrm{E}+20$ & $2.31 \mathrm{E}-03$ & $1.33 \mathrm{E}+20$ & $2.48 \mathrm{E}-03$ & $1.44 \mathrm{E}+20$ & $2.69 \mathrm{E}-03$ \\
\hline $1.50 \mathrm{E}-02$ & 1.156 & $1.03 E+20$ & $1.49 \mathrm{E}-03$ & $1.10 \mathrm{E}+20$ & $1.59 \mathrm{E}-03$ & $1.16 \mathrm{E}+20$ & $1.67 \mathrm{E}-03$ & $1.21 \mathrm{E}+20$ & $1.75 \mathrm{E}-03$ \\
\hline $1.90 \mathrm{E}-02$ & 0.973 & $1.06 \mathrm{E}+20$ & $1.29 \mathrm{E}-03$ & $1.13 \mathrm{E}+20$ & 1.37E-03 & $1.26 \mathrm{E}+20$ & $1.53 \mathrm{E}-03$ & $1.26 \mathrm{E}+20$ & $1.54 \mathrm{E}-03$ \\
\hline $2.55 \mathrm{E}-02$ & 0.555 & $1.50 \mathrm{E}+20$ & $1.04 \mathrm{E}-03$ & $1.65 \mathrm{E}+20$ & $1.14 \mathrm{E}-03$ & $1.82 \mathrm{E}+20$ & $1.27 \mathrm{E}-03$ & $1.89 \mathrm{E}+20$ & $1.31 \mathrm{E}-03$ \\
\hline $3.20 \mathrm{E}-02$ & 26.762 & $1.39 \mathrm{E}+20$ & 4.66E-02 & $1.51 \mathrm{E}+20$ & $5.06 \mathrm{E}-02$ & $1.59 \mathrm{E}+20$ & $5.32 \mathrm{E}-02$ & $1.68 \mathrm{E}+20$ & 5.64E-02 \\
\hline 4.00E-02 & 8.161 & $6.89 \mathrm{E}+19$ & 7.03E-03 & $7.48 \mathrm{E}+19$ & 7.63E-03 & $7.69 \mathrm{E}+19$ & $7.84 \mathrm{E}-03$ & $7.96 \mathrm{E}+19$ & 8.12E-03 \\
\hline $5.25 \mathrm{E}-02$ & 6.515 & $1.32 \mathrm{E}+20$ & $1.07 \mathrm{E}-02$ & $1.48 \mathrm{E}+20$ & $1.20 \mathrm{E}-02$ & $1.56 \mathrm{E}+20$ & $1.27 \mathrm{E}-02$ & $1.62 \mathrm{E}+20$ & $1.32 \mathrm{E}-02$ \\
\hline $6.60 \mathrm{E}-02$ & 6.576 & $1.35 \mathrm{E}+20$ & $1.11 \mathrm{E}-02$ & $1.46 \mathrm{E}+20$ & $1.20 \mathrm{E}-02$ & $1.55 \mathrm{E}+20$ & $1.28 \mathrm{E}-02$ & $1.62 \mathrm{E}+20$ & $1.33 \mathrm{E}-02$ \\
\hline $8.80 \mathrm{E}-02$ & 13.137 & $1.98 \mathrm{E}+20$ & $3.25 \mathrm{E}-02$ & $2.13 E+20$ & $3.50 \mathrm{E}-02$ & $2.34 \mathrm{E}+20$ & $3.84 \mathrm{E}-02$ & $2.41 \mathrm{E}+20$ & $3.95 \mathrm{E}-02$ \\
\hline 1.10E-01 & 11.84 & $1.24 \mathrm{E}+20$ & $1.84 \mathrm{E}-02$ & $1.33 \mathrm{E}+20$ & 1.97E-02 & $1.42 \mathrm{E}+20$ & 2.11E-02 & $1.50 \mathrm{E}+20$ & $2.23 \mathrm{E}-02$ \\
\hline $1.35 \mathrm{E}-01$ & 11.27 & $1.82 \mathrm{E}+20$ & $2.57 \mathrm{E}-02$ & $1.96 \mathrm{E}+20$ & $2.76 \mathrm{E}-02$ & $2.12 \mathrm{E}+20$ & $2.98 \mathrm{E}-02$ & $2.22 \mathrm{E}+20$ & $3.12 \mathrm{E}-02$ \\
\hline $1.60 \mathrm{E}-01$ & 21.53 & $1.07 E+20$ & $2.88 \mathrm{E}-02$ & $1.13 E+20$ & 3.03E-02 & $1.25 \mathrm{E}+20$ & $3.35 \mathrm{E}-02$ & $1.28 \mathrm{E}+20$ & $3.43 \mathrm{E}-02$ \\
\hline 1.90E-01 & 15.85 & $1.45 \mathrm{E}+20$ & 2.87E-02 & $1.60 \mathrm{E}+20$ & 3.17E-02 & $1.72 \mathrm{E}+20$ & $3.41 \mathrm{E}-02$ & $1.79 \mathrm{E}+20$ & $3.55 \mathrm{E}-02$ \\
\hline $2.20 \mathrm{E}-01$ & 22.01 & $1.15 E+20$ & 3.17E-02 & $1.31 \mathrm{E}+20$ & 3.60E-02 & $1.40 \mathrm{E}+20$ & $3.84 \mathrm{E}-02$ & $1.48 \mathrm{E}+20$ & 4.07E-02 \\
\hline
\end{tabular}




\begin{tabular}{|c|c|c|c|c|c|c|c|c|c|}
\hline \multirow{2}{*}{$\begin{array}{c}\text { Upper } \\
\text { Energy(MeV) }\end{array}$} & \multirow{2}{*}{$\begin{array}{l}\text { DPA xsec } \\
\text { keV-barn }\end{array}$} & \multicolumn{2}{|c|}{$9-4$} & \multicolumn{2}{|c|}{$9-3$} & \multicolumn{2}{|c|}{$9-2$} & \multicolumn{2}{|c|}{$9-1$} \\
\hline & & Total Fluence & DPA & Total Fluence & DPA & Total Fluence & DPA & Total Fluence & DPA \\
\hline $2.55 \mathrm{E}-01$ & 18.27 & $1.35 \mathrm{E}+20$ & $3.08 \mathrm{E}-02$ & $1.49 E+20$ & $3.40 \mathrm{E}-02$ & $1.66 \mathrm{E}+20$ & 3.80E-02 & $1.70 \mathrm{E}+20$ & 3.89E-02 \\
\hline $2.90 \mathrm{E}-01$ & 17.37 & $1.50 \mathrm{E}+20$ & $3.25 \mathrm{E}-02$ & $1.67 \mathrm{E}+20$ & 3.62E-02 & $1.77 \mathrm{E}+20$ & $3.84 \mathrm{E}-02$ & $1.84 \mathrm{E}+20$ & $3.99 \mathrm{E}-02$ \\
\hline 3.20E-01 & 13.51 & $1.01 \mathrm{E}+20$ & $1.71 \mathrm{E}-02$ & $1.14 \mathrm{E}+20$ & $1.92 \mathrm{E}-02$ & $1.21 \mathrm{E}+20$ & $2.05 \mathrm{E}-02$ & $1.33 \mathrm{E}+20$ & $2.25 \mathrm{E}-02$ \\
\hline $3.60 \mathrm{E}-01$ & 25.14 & $1.48 \mathrm{E}+20$ & 4.67E-02 & $1.62 \mathrm{E}+20$ & $5.08 \mathrm{E}-02$ & $1.75 \mathrm{E}+20$ & 5.51E-02 & $1.83 \mathrm{E}+20$ & $5.74 \mathrm{E}-02$ \\
\hline 4.00E-01 & 46.52 & $1.28 \mathrm{E}+20$ & 7.46E-02 & $1.36 \mathrm{E}+20$ & 7.93E-02 & $1.51 \mathrm{E}+20$ & 8.76E-02 & $1.53 \mathrm{E}+20$ & $8.88 \mathrm{E}-02$ \\
\hline 4.50E-01 & 43.04 & $1.16 \mathrm{E}+20$ & $6.22 \mathrm{E}-02$ & $1.34 \mathrm{E}+20$ & $7.22 \mathrm{E}-02$ & $1.43 E+20$ & 7.70E-02 & $1.46 \mathrm{E}+20$ & $7.86 \mathrm{E}-02$ \\
\hline 5.00E-01 & 36.4 & $1.38 \mathrm{E}+20$ & $6.28 \mathrm{E}-02$ & $1.48 \mathrm{E}+20$ & $6.75 \mathrm{E}-02$ & $1.63 \mathrm{E}+20$ & 7.41E-02 & $1.68 \mathrm{E}+20$ & $7.66 \mathrm{E}-02$ \\
\hline 5.50E-01 & 33.22 & $1.35 \mathrm{E}+20$ & $5.61 \mathrm{E}-02$ & $1.51 E+20$ & $6.29 \mathrm{E}-02$ & $1.57 \mathrm{E}+20$ & $6.54 \mathrm{E}-02$ & $1.62 \mathrm{E}+20$ & $6.74 \mathrm{E}-02$ \\
\hline $6.00 \mathrm{E}-01$ & 34.18 & $1.30 \mathrm{E}+20$ & $5.55 \mathrm{E}-02$ & $1.44 \mathrm{E}+20$ & $6.13 \mathrm{E}-02$ & $1.53 \mathrm{E}+20$ & $6.52 \mathrm{E}-02$ & $1.62 \mathrm{E}+20$ & $6.90 \mathrm{E}-02$ \\
\hline $6.60 \mathrm{E}-01$ & 19.64 & $1.54 \mathrm{E}+20$ & 3.78E-02 & $1.75 \mathrm{E}+20$ & 4.30E-02 & $1.92 \mathrm{E}+20$ & 4.72E-02 & $1.98 \mathrm{E}+20$ & 4.85E-02 \\
\hline $7.20 \mathrm{E}-01$ & 48.89 & $1.62 \mathrm{E}+20$ & $9.90 \mathrm{E}-02$ & $1.80 \mathrm{E}+20$ & 1.10E-01 & $1.93 E+20$ & $1.18 \mathrm{E}-01$ & $2.05 \mathrm{E}+20$ & 1.25E-01 \\
\hline 7.80E-01 & 74.22 & $1.57 \mathrm{E}+20$ & $1.46 \mathrm{E}-01$ & $1.71 \mathrm{E}+20$ & $1.59 \mathrm{E}-01$ & $1.82 \mathrm{E}+20$ & $1.69 \mathrm{E}-01$ & $1.89 \mathrm{E}+20$ & $1.75 \mathrm{E}-01$ \\
\hline 8.40E-01 & 44.02 & $1.18 \mathrm{E}+20$ & $6.48 \mathrm{E}-02$ & $1.37 \mathrm{E}+20$ & 7.56E-02 & $1.44 \mathrm{E}+20$ & 7.91E-02 & $1.50 \mathrm{E}+20$ & $8.24 \mathrm{E}-02$ \\
\hline 9.20E-01 & 40.97 & $1.51 \mathrm{E}+20$ & 7.73E-02 & $1.60 \mathrm{E}+20$ & $8.21 \mathrm{E}-02$ & $1.72 \mathrm{E}+20$ & $8.82 \mathrm{E}-02$ & $1.86 \mathrm{E}+20$ & $9.51 \mathrm{E}-02$ \\
\hline $1.00 \mathrm{E}+00$ & 50.95 & $1.37 \mathrm{E}+20$ & 8.71E-02 & $1.49 \mathrm{E}+20$ & $9.50 \mathrm{E}-02$ & $1.58 \mathrm{E}+20$ & $1.01 \mathrm{E}-01$ & $1.66 \mathrm{E}+20$ & $1.06 \mathrm{E}-01$ \\
\hline $1.20 \mathrm{E}+00$ & 50.09 & $3.25 E+20$ & $2.04 \mathrm{E}-01$ & $3.43 E+20$ & 2.15E-01 & $3.77 E+20$ & $2.36 \mathrm{E}-01$ & $3.95 E+20$ & 2.47E-01 \\
\hline $1.40 \mathrm{E}+00$ & 64.54 & $2.81 \mathrm{E}+20$ & $2.26 \mathrm{E}-01$ & $3.09 \mathrm{E}+20$ & $2.49 \mathrm{E}-01$ & $3.40 \mathrm{E}+20$ & $2.74 \mathrm{E}-01$ & $3.46 \mathrm{E}+20$ & 2.79E-01 \\
\hline $1.60 \mathrm{E}+00$ & 73.58 & $2.41 \mathrm{E}+20$ & $2.22 \mathrm{E}-01$ & $2.73 E+20$ & $2.51 \mathrm{E}-01$ & $2.86 \mathrm{E}+20$ & 2.63E-01 & $2.97 \mathrm{E}+20$ & $2.73 \mathrm{E}-01$ \\
\hline $1.80 \mathrm{E}+00$ & 76.46 & $2.15 \mathrm{E}+20$ & $2.05 \mathrm{E}-01$ & $2.24 \mathrm{E}+20$ & $2.14 \mathrm{E}-01$ & $2.50 \mathrm{E}+20$ & $2.39 \mathrm{E}-01$ & $2.60 \mathrm{E}+20$ & $2.48 \mathrm{E}-01$ \\
\hline $2.00 \mathrm{E}+00$ & 95.15 & $1.85 \mathrm{E}+20$ & $2.20 \mathrm{E}-01$ & $2.01 E+20$ & 2.39E-01 & $2.19 E+20$ & 2.60E-01 & $2.34 \mathrm{E}+20$ & 2.79E-01 \\
\hline $2.30 \mathrm{E}+00$ & 93.75 & $2.34 \mathrm{E}+20$ & $2.74 \mathrm{E}-01$ & $2.51 \mathrm{E}+20$ & 2.94E-01 & $2.74 \mathrm{E}+20$ & $3.21 \mathrm{E}-01$ & $2.94 \mathrm{E}+20$ & $3.45 \mathrm{E}-01$ \\
\hline $2.60 \mathrm{E}+00$ & 112.05 & $2.12 \mathrm{E}+20$ & 2.97E-01 & $2.34 \mathrm{E}+20$ & $3.28 \mathrm{E}-01$ & $2.53 E+20$ & $3.55 \mathrm{E}-01$ & $2.64 \mathrm{E}+20$ & 3.70E-01 \\
\hline $2.90 \mathrm{E}+00$ & 123.55 & $1.66 \mathrm{E}+20$ & $2.57 \mathrm{E}-01$ & $1.79 \mathrm{E}+20$ & $2.76 \mathrm{E}-01$ & $1.91 \mathrm{E}+20$ & 2.96E-01 & $1.96 \mathrm{E}+20$ & 3.03E-01 \\
\hline $3.30 \mathrm{E}+00$ & 133.45 & $1.63 \mathrm{E}+20$ & $2.72 \mathrm{E}-01$ & $1.79 \mathrm{E}+20$ & 2.99E-01 & $1.88 \mathrm{E}+20$ & 3.13E-01 & $2.00 \mathrm{E}+20$ & 3.34E-01 \\
\hline $3.70 \mathrm{E}+00$ & 135.25 & $1.13 \mathrm{E}+20$ & 1.91E-01 & $1.20 \mathrm{E}+20$ & 2.03E-01 & $1.31 E+20$ & $2.22 \mathrm{E}-01$ & $1.35 \mathrm{E}+20$ & $2.28 \mathrm{E}-01$ \\
\hline $4.10 \mathrm{E}+00$ & 149.55 & $8.60 \mathrm{E}+19$ & $1.61 \mathrm{E}-01$ & $9.42 \mathrm{E}+19$ & $1.76 \mathrm{E}-01$ & $9.98 \mathrm{E}+19$ & $1.86 \mathrm{E}-01$ & $1.06 \mathrm{E}+20$ & $1.99 \mathrm{E}-01$ \\
\hline $4.50 \mathrm{E}+00$ & 158.25 & $6.78 \mathrm{E}+19$ & $1.34 \mathrm{E}-01$ & $7.14 \mathrm{E}+19$ & $1.41 \mathrm{E}-01$ & $7.68 \mathrm{E}+19$ & $1.52 \mathrm{E}-01$ & $8.19 E+19$ & $1.62 \mathrm{E}-01$ \\
\hline $5.00 \mathrm{E}+00$ & 168.55 & $6.01 \mathrm{E}+19$ & 1.27E-01 & $6.78 \mathrm{E}+19$ & 1.43E-01 & $7.42 E+19$ & $1.56 \mathrm{E}-01$ & $7.57 \mathrm{E}+19$ & $1.60 \mathrm{E}-01$ \\
\hline $5.50 \mathrm{E}+00$ & 176.46 & $4.50 E+19$ & $9.92 \mathrm{E}-02$ & $4.52 E+19$ & 9.96E-02 & $4.86 E+19$ & 1.07E-01 & $5.15 E+19$ & $1.14 \mathrm{E}-01$ \\
\hline
\end{tabular}




\begin{tabular}{|c|c|c|c|c|c|c|c|c|c|}
\hline \multirow{2}{*}{$\begin{array}{c}\text { Upper } \\
\text { Energy(MeV) }\end{array}$} & \multirow{2}{*}{$\begin{array}{l}\text { DPA xsec } \\
\text { keV-barn }\end{array}$} & \multicolumn{2}{|c|}{$9-4$} & \multicolumn{2}{|c|}{$9-3$} & \multicolumn{2}{|c|}{$9-2$} & \multicolumn{2}{|c|}{$9-1$} \\
\hline & & Total Fluence & DPA & Total Fluence & DPA & Total Fluence & DPA & Total Fluence & DPA \\
\hline $6.00 E+00$ & 183.06 & $3.02 E+19$ & $6.92 \mathrm{E}-02$ & $3.15 E+19$ & $7.21 \mathrm{E}-02$ & $3.38 \mathrm{E}+19$ & 7.73E-02 & $3.58 \mathrm{E}+19$ & $8.20 \mathrm{E}-02$ \\
\hline $6.70 E+00$ & 189.26 & $2.76 \mathrm{E}+19$ & $6.52 \mathrm{E}-02$ & $3.01 \mathrm{E}+19$ & $7.11 \mathrm{E}-02$ & $3.12 E+19$ & 7.39E-02 & $3.33 E+19$ & $7.88 \mathrm{E}-02$ \\
\hline $7.40 \mathrm{E}+00$ & 196.67 & $1.53 \mathrm{E}+19$ & $3.76 \mathrm{E}-02$ & $1.73 \mathrm{E}+19$ & $4.25 \mathrm{E}-02$ & $1.89 \mathrm{E}+19$ & 4.66E-02 & $2.12 E+19$ & $5.21 \mathrm{E}-02$ \\
\hline $8.20 \mathrm{E}+00$ & 203.37 & $9.59 \mathrm{E}+18$ & $2.44 \mathrm{E}-02$ & $1.07 \mathrm{E}+19$ & $2.73 \mathrm{E}-02$ & $1.11 \mathrm{E}+19$ & $2.83 \mathrm{E}-02$ & $1.23 \mathrm{E}+19$ & $3.12 \mathrm{E}-02$ \\
\hline $9.00 \mathrm{E}+00$ & 214.58 & $5.16 \mathrm{E}+18$ & $1.38 \mathrm{E}-02$ & $5.59 \mathrm{E}+18$ & $1.50 \mathrm{E}-02$ & $6.35 \mathrm{E}+18$ & $1.70 \mathrm{E}-02$ & $7.54 \mathrm{E}+18$ & $2.02 \mathrm{E}-02$ \\
\hline $1.00 \mathrm{E}+01$ & 225.69 & $3.86 \mathrm{E}+18$ & $1.09 \mathrm{E}-02$ & $3.68 \mathrm{E}+18$ & $1.04 \mathrm{E}-02$ & $3.54 \mathrm{E}+18$ & $9.99 \mathrm{E}-03$ & $4.46 \mathrm{E}+18$ & $1.26 \mathrm{E}-02$ \\
\hline $1.10 \mathrm{E}+01$ & 237.6 & $1.61 \mathrm{E}+18$ & 4.77E-03 & $1.35 \mathrm{E}+18$ & $4.00 \mathrm{E}-03$ & $1.84 \mathrm{E}+18$ & $5.46 \mathrm{E}-03$ & $2.47 \mathrm{E}+18$ & 7.35E-03 \\
\hline $1.20 \mathrm{E}+01$ & 247.41 & $5.70 \mathrm{E}+17$ & $1.76 \mathrm{E}-03$ & $9.36 \mathrm{E}+17$ & $2.89 \mathrm{E}-03$ & $9.63 E+17$ & $2.98 \mathrm{E}-03$ & $9.09 E+17$ & $2.81 \mathrm{E}-03$ \\
\hline $1.30 \mathrm{E}+01$ & 258.52 & $3.43 \mathrm{E}+17$ & $1.11 \mathrm{E}-03$ & $3.34 \mathrm{E}+17$ & $1.08 \mathrm{E}-03$ & $3.88 \mathrm{E}+17$ & $1.25 \mathrm{E}-03$ & $4.01 \mathrm{E}+17$ & $1.30 \mathrm{E}-03$ \\
\hline $1.40 \mathrm{E}+01$ & 271.35 & $1.99 \mathrm{E}+17$ & $6.74 \mathrm{E}-04$ & $1.11 \mathrm{E}+17$ & $3.78 \mathrm{E}-04$ & $4.49 E+16$ & $1.52 \mathrm{E}-04$ & $6.06 \mathrm{E}+16$ & $2.05 \mathrm{E}-04$ \\
\hline $1.50 \mathrm{E}+01$ & 290.27 & $3.77 \mathrm{E}+16$ & $1.37 \mathrm{E}-04$ & $1.27 \mathrm{E}+17$ & $4.62 \mathrm{E}-04$ & 7.74E+16 & $2.81 \mathrm{E}-04$ & $1.00 \mathrm{E}+17$ & $3.64 \mathrm{E}-04$ \\
\hline $1.60 \mathrm{E}+01$ & 293.2 & $1.36 \mathrm{E}+16$ & 4.99E-05 & $3.59 \mathrm{E}+16$ & $1.32 \mathrm{E}-04$ & $1.39 \mathrm{E}+16$ & 5.11E-05 & 4.67E+15 & $1.71 \mathrm{E}-05$ \\
\hline $1.70 \mathrm{E}+01$ & 292.73 & $6.89 \mathrm{E}+16$ & $2.52 \mathrm{E}-04$ & $1.30 \mathrm{E}+16$ & 4.74E-05 & $8.80 \mathrm{E}+15$ & $3.22 \mathrm{E}-05$ & $0.00 \mathrm{E}+00$ & $0.00 \mathrm{E}+00$ \\
\hline $1.80 \mathrm{E}+01$ & 297.65 & $0.00 \mathrm{E}+00$ & $0.00 \mathrm{E}+00$ & $0.00 \mathrm{E}+00$ & $0.00 \mathrm{E}+00$ & $0.00 \mathrm{E}+00$ & $0.00 \mathrm{E}+00$ & $0.00 \mathrm{E}+00$ & $0.00 \mathrm{E}+00$ \\
\hline $1.90 \mathrm{E}+01$ & 307.26 & $0.00 \mathrm{E}+00$ & $0.00 \mathrm{E}+00$ & $0.00 \mathrm{E}+00$ & $0.00 \mathrm{E}+00$ & $0.00 \mathrm{E}+00$ & $0.00 \mathrm{E}+00$ & $0.00 \mathrm{E}+00$ & $0.00 \mathrm{E}+00$ \\
\hline \multirow[t]{2}{*}{$2.00 \mathrm{E}+01$} & 316.36 & $0.00 \mathrm{E}+00$ & $0.00 \mathrm{E}+00$ & $2.95 \mathrm{E}+16$ & 1.17E-04 & $0.00 \mathrm{E}+00$ & $0.00 \mathrm{E}+00$ & $0.00 \mathrm{E}+00$ & $0.00 \mathrm{E}+00$ \\
\hline & & & $4.36 \mathrm{E}+00$ & & $4.74 \mathrm{E}+00$ & & $5.10 \mathrm{E}+00$ & & $5.35 E+00$ \\
\hline
\end{tabular}


Table 13. Calculated fluence and DPA for capsule 8 of the UCSB experiment.

\begin{tabular}{|c|c|c|c|}
\hline \multirow[b]{2}{*}{$\begin{array}{c}\text { Upper } \\
\text { Energy(MeV) }\end{array}$} & \multirow{2}{*}{$\begin{array}{l}\text { DPA xsec } \\
\text { keV-barn }\end{array}$} & \multicolumn{2}{|c|}{$8-1$} \\
\hline & & Total Fluence & DPA \\
\hline $1.00 \mathrm{E}-10$ & 0 & $1.20 \mathrm{E}+15$ & \\
\hline $1.00 \mathrm{E}-09$ & 7.73 & $7.54 \mathrm{E}+17$ & $7.29 \mathrm{E}-05$ \\
\hline $1.00 \mathrm{E}-08$ & 2.444 & $1.14 \mathrm{E}+20$ & $3.47 \mathrm{E}-03$ \\
\hline $2.30 \mathrm{E}-08$ & 1.278 & $4.11 \mathrm{E}+20$ & $6.56 \mathrm{E}-03$ \\
\hline $5.00 \mathrm{E}-08$ & 0.857 & $1.03 E+21$ & 1.10E-02 \\
\hline $7.60 \mathrm{E}-08$ & 0.644 & $7.34 \mathrm{E}+20$ & $5.91 \mathrm{E}-03$ \\
\hline $1.15 \mathrm{E}-07$ & 0.523 & $6.30 E+20$ & 4.12E-03 \\
\hline $1.70 \mathrm{E}-07$ & 0.428 & $3.93 E+20$ & $2.10 \mathrm{E}-03$ \\
\hline $2.55 \mathrm{E}-07$ & 0.351 & $2.75 \mathrm{E}+20$ & $1.21 \mathrm{E}-03$ \\
\hline $3.80 \mathrm{E}-07$ & 0.287 & $2.31 E+20$ & $8.28 \mathrm{E}-04$ \\
\hline $5.50 \mathrm{E}-07$ & 0.237 & $2.07 \mathrm{E}+20$ & $6.14 \mathrm{E}-04$ \\
\hline $8.40 \mathrm{E}-07$ & 0.194 & $2.32 \mathrm{E}+20$ & $5.63 \mathrm{E}-04$ \\
\hline $1.28 \mathrm{E}-06$ & 0.157 & $2.24 \mathrm{E}+20$ & 4.40E-04 \\
\hline $1.90 \mathrm{E}-06$ & 0.128 & $2.09 \mathrm{E}+20$ & $3.34 \mathrm{E}-04$ \\
\hline $2.80 \mathrm{E}-06$ & 0.105 & $2.04 \mathrm{E}+20$ & 2.67E-04 \\
\hline 4.25E-06 & 0.086 & $2.15 E+20$ & $2.31 \mathrm{E}-04$ \\
\hline $6.30 \mathrm{E}-06$ & 0.07 & 1.97E+20 & $1.73 \mathrm{E}-04$ \\
\hline $9.20 \mathrm{E}-06$ & 0.058 & $1.91 \mathrm{E}+20$ & $1.38 \mathrm{E}-04$ \\
\hline $1.35 \mathrm{E}-05$ & 0.048 & $2.00 \mathrm{E}+20$ & 1.20E-04 \\
\hline $2.10 \mathrm{E}-05$ & 0.038 & $2.31 \mathrm{E}+20$ & $1.10 \mathrm{E}-04$ \\
\hline $3.00 \mathrm{E}-05$ & 0.031 & $1.89 \mathrm{E}+20$ & 7.32E-05 \\
\hline 4.50E-05 & 0.026 & $2.13 E+20$ & $6.93 \mathrm{E}-05$ \\
\hline $6.90 \mathrm{E}-05$ & 0.021 & $2.30 \mathrm{E}+20$ & $6.03 \mathrm{E}-05$ \\
\hline $1.00 \mathrm{E}-04$ & 0.017 & $2.03 E+20$ & 4.31E-05 \\
\hline $1.35 \mathrm{E}-04$ & 0.014 & $1.65 E+20$ & $2.89 \mathrm{E}-05$ \\
\hline $1.70 \mathrm{E}-04$ & 0.014 & $1.29 E+20$ & $2.26 \mathrm{E}-05$ \\
\hline $2.20 \mathrm{E}-04$ & 0.011 & $1.44 \mathrm{E}+20$ & $1.98 \mathrm{E}-05$ \\
\hline
\end{tabular}




\begin{tabular}{|c|c|c|c|}
\hline \multirow{2}{*}{$\begin{array}{c}\text { Upper } \\
\text { Energy(MeV) }\end{array}$} & \multirow{2}{*}{$\begin{array}{l}\text { DPA xsec } \\
\text { keV-barn }\end{array}$} & \multicolumn{2}{|c|}{$8-1$} \\
\hline & & Total Fluence & DPA \\
\hline $2.80 \mathrm{E}-04$ & 0.011 & $1.35 \mathrm{E}+20$ & $1.85 \mathrm{E}-05$ \\
\hline $3.60 \mathrm{E}-04$ & 0.01 & $1.41 \mathrm{E}+20$ & $1.76 \mathrm{E}-05$ \\
\hline $4.50 \mathrm{E}-04$ & 0.007 & $1.24 \mathrm{E}+20$ & $1.08 \mathrm{E}-05$ \\
\hline $5.75 \mathrm{E}-04$ & 0.005 & $1.37 \mathrm{E}+20$ & $8.55 \mathrm{E}-06$ \\
\hline $7.60 \mathrm{E}-04$ & 0.129 & $1.56 \mathrm{E}+20$ & $2.51 \mathrm{E}-04$ \\
\hline $9.60 \mathrm{E}-04$ & 0.308 & $1.33 \mathrm{E}+20$ & 5.11E-04 \\
\hline $1.28 \mathrm{E}-03$ & 0.672 & $1.59 \mathrm{E}+20$ & $1.34 \mathrm{E}-03$ \\
\hline $1.60 \mathrm{E}-03$ & 0.477 & $1.29 \mathrm{E}+20$ & 7.67E-04 \\
\hline $2.00 \mathrm{E}-03$ & 0.511 & $1.30 \mathrm{E}+20$ & 8.32E-04 \\
\hline $2.70 \mathrm{E}-03$ & 0.568 & $1.73 E+20$ & $1.23 \mathrm{E}-03$ \\
\hline $3.40 \mathrm{E}-03$ & 0.632 & $1.37 \mathrm{E}+20$ & $1.08 \mathrm{E}-03$ \\
\hline 4.50E-03 & 0.822 & $1.66 \mathrm{E}+20$ & $1.70 \mathrm{E}-03$ \\
\hline $5.50 \mathrm{E}-03$ & 0.826 & $1.26 \mathrm{E}+20$ & $1.30 \mathrm{E}-03$ \\
\hline 7.20E-03 & 1.671 & $1.65 E+20$ & $3.44 \mathrm{E}-03$ \\
\hline $9.20 \mathrm{E}-03$ & 3.709 & $1.28 \mathrm{E}+20$ & 5.93E-03 \\
\hline $1.20 \mathrm{E}-02$ & 1.491 & $1.57 \mathrm{E}+20$ & 2.93E-03 \\
\hline $1.50 \mathrm{E}-02$ & 1.156 & $1.36 \mathrm{E}+20$ & $1.96 \mathrm{E}-03$ \\
\hline $1.90 \mathrm{E}-02$ & 0.973 & $1.46 \mathrm{E}+20$ & $1.78 \mathrm{E}-03$ \\
\hline $2.55 \mathrm{E}-02$ & 0.555 & $2.18 \mathrm{E}+20$ & $1.51 \mathrm{E}-03$ \\
\hline $3.20 \mathrm{E}-02$ & 26.762 & $1.90 \mathrm{E}+20$ & $6.36 \mathrm{E}-02$ \\
\hline 4.00E-02 & 8.161 & $9.32 \mathrm{E}+19$ & 9.51E-03 \\
\hline $5.25 \mathrm{E}-02$ & 6.515 & $1.87 \mathrm{E}+20$ & $1.52 \mathrm{E}-02$ \\
\hline $6.60 \mathrm{E}-02$ & 6.576 & $1.84 \mathrm{E}+20$ & $1.51 \mathrm{E}-02$ \\
\hline $8.80 \mathrm{E}-02$ & 13.137 & $2.79 E+20$ & 4.58E-02 \\
\hline $1.10 \mathrm{E}-01$ & 11.84 & $1.70 \mathrm{E}+20$ & $2.52 \mathrm{E}-02$ \\
\hline $1.35 \mathrm{E}-01$ & 11.27 & $2.49 \mathrm{E}+20$ & 3.51E-02 \\
\hline $1.60 \mathrm{E}-01$ & 21.53 & $1.43 \mathrm{E}+20$ & $3.85 \mathrm{E}-02$ \\
\hline $1.90 \mathrm{E}-01$ & 15.85 & $2.02 \mathrm{E}+20$ & 4.01E-02 \\
\hline $2.20 \mathrm{E}-01$ & 22.01 & $1.63 \mathrm{E}+20$ & 4.48E-02 \\
\hline
\end{tabular}




\begin{tabular}{|c|c|c|c|}
\hline \multirow{2}{*}{$\begin{array}{c}\text { Upper } \\
\text { Energy(MeV) }\end{array}$} & \multirow{2}{*}{$\begin{array}{l}\text { DPA xsec } \\
\text { keV-barn }\end{array}$} & \multicolumn{2}{|c|}{$8-1$} \\
\hline & & Total Fluence & DPA \\
\hline $2.55 \mathrm{E}-01$ & 18.27 & $1.94 \mathrm{E}+20$ & $4.42 \mathrm{E}-02$ \\
\hline $2.90 \mathrm{E}-01$ & 17.37 & $2.08 \mathrm{E}+20$ & $4.52 \mathrm{E}-02$ \\
\hline $3.20 \mathrm{E}-01$ & 13.51 & $1.48 \mathrm{E}+20$ & $2.51 \mathrm{E}-02$ \\
\hline $3.60 \mathrm{E}-01$ & 25.14 & $2.08 \mathrm{E}+20$ & $6.53 \mathrm{E}-02$ \\
\hline 4.00E-01 & 46.52 & 1.77E+20 & $1.03 \mathrm{E}-01$ \\
\hline $4.50 \mathrm{E}-01$ & 43.04 & $1.68 \mathrm{E}+20$ & $9.02 \mathrm{E}-02$ \\
\hline $5.00 \mathrm{E}-01$ & 36.4 & $1.90 \mathrm{E}+20$ & 8.66E-02 \\
\hline $5.50 \mathrm{E}-01$ & 33.22 & $1.92 \mathrm{E}+20$ & 7.95E-02 \\
\hline $6.00 \mathrm{E}-01$ & 34.18 & $1.83 \mathrm{E}+20$ & $7.80 \mathrm{E}-02$ \\
\hline $6.60 \mathrm{E}-01$ & 19.64 & $2.28 \mathrm{E}+20$ & 5.59E-02 \\
\hline 7.20E-01 & 48.89 & $2.33 \mathrm{E}+20$ & $1.42 \mathrm{E}-01$ \\
\hline $7.80 \mathrm{E}-01$ & 74.22 & $2.17 \mathrm{E}+20$ & $2.02 \mathrm{E}-01$ \\
\hline $8.40 \mathrm{E}-01$ & 44.02 & $1.70 \mathrm{E}+20$ & $9.34 \mathrm{E}-02$ \\
\hline $9.20 \mathrm{E}-01$ & 40.97 & $2.07 \mathrm{E}+20$ & $1.06 \mathrm{E}-01$ \\
\hline $1.00 \mathrm{E}+00$ & 50.95 & $1.89 \mathrm{E}+20$ & $1.21 \mathrm{E}-01$ \\
\hline $1.20 \mathrm{E}+00$ & 50.09 & $4.41 E+20$ & $2.76 \mathrm{E}-01$ \\
\hline $1.40 \mathrm{E}+00$ & 64.54 & $3.98 \mathrm{E}+20$ & $3.21 \mathrm{E}-01$ \\
\hline $1.60 \mathrm{E}+00$ & 73.58 & $3.42 \mathrm{E}+20$ & $3.15 \mathrm{E}-01$ \\
\hline $1.80 \mathrm{E}+00$ & 76.46 & $2.95 \mathrm{E}+20$ & $2.82 \mathrm{E}-01$ \\
\hline $2.00 \mathrm{E}+00$ & 95.15 & $2.56 \mathrm{E}+20$ & $3.05 \mathrm{E}-01$ \\
\hline $2.30 \mathrm{E}+00$ & 93.75 & $3.23 \mathrm{E}+20$ & 3.79E-01 \\
\hline $2.60 \mathrm{E}+00$ & 112.05 & $2.98 \mathrm{E}+20$ & 4.17E-01 \\
\hline $2.90 \mathrm{E}+00$ & 123.55 & $2.26 \mathrm{E}+20$ & $3.49 \mathrm{E}-01$ \\
\hline $3.30 \mathrm{E}+00$ & 133.45 & $2.24 \mathrm{E}+20$ & 3.73E-01 \\
\hline $3.70 \mathrm{E}+00$ & 135.25 & $1.52 \mathrm{E}+20$ & $2.56 \mathrm{E}-01$ \\
\hline $4.10 \mathrm{E}+00$ & 149.55 & $1.18 \mathrm{E}+20$ & 2.20E-01 \\
\hline $4.50 \mathrm{E}+00$ & 158.25 & $9.00 \mathrm{E}+19$ & $1.78 \mathrm{E}-01$ \\
\hline $5.00 \mathrm{E}+00$ & 168.55 & $8.75 E+19$ & $1.84 \mathrm{E}-01$ \\
\hline $5.50 \mathrm{E}+00$ & 176.46 & $5.99 E+19$ & $1.32 \mathrm{E}-01$ \\
\hline
\end{tabular}




\begin{tabular}{|c|c|c|c|}
\hline & DPA xsec & \multicolumn{2}{|c|}{$8-1$} \\
\hline $\begin{array}{c}\text { Upper } \\
\text { Energy(MeV) }\end{array}$ & keV-barn & Total Fluence & DPA \\
\hline $6.00 \mathrm{E}+00$ & 183.06 & $4.07 \mathrm{E}+19$ & $9.32 \mathrm{E}-02$ \\
\hline $6.70 \mathrm{E}+00$ & 189.26 & $3.71 \mathrm{E}+19$ & $8.77 \mathrm{E}-02$ \\
\hline $7.40 \mathrm{E}+00$ & 196.67 & $2.18 \mathrm{E}+19$ & $5.36 \mathrm{E}-02$ \\
\hline $8.20 \mathrm{E}+00$ & 203.37 & $1.36 \mathrm{E}+19$ & $3.45 \mathrm{E}-02$ \\
\hline $9.00 \mathrm{E}+00$ & 214.58 & $7.65 \mathrm{E}+18$ & $2.05 \mathrm{E}-02$ \\
\hline $1.00 \mathrm{E}+01$ & 225.69 & $4.36 \mathrm{E}+18$ & $1.23 \mathrm{E}-02$ \\
\hline $1.10 \mathrm{E}+01$ & 237.6 & $2.27 \mathrm{E}+18$ & $6.73 \mathrm{E}-03$ \\
\hline $1.20 \mathrm{E}+01$ & 247.41 & $8.85 \mathrm{E}+17$ & $2.74 \mathrm{E}-03$ \\
\hline $1.30 \mathrm{E}+01$ & 258.52 & $3.53 \mathrm{E}+17$ & $1.14 \mathrm{E}-03$ \\
\hline $1.40 \mathrm{E}+01$ & 271.35 & $1.84 \mathrm{E}+17$ & $6.23 \mathrm{E}-04$ \\
\hline $1.50 \mathrm{E}+01$ & 290.27 & $9.44 \mathrm{E}+16$ & $3.43 \mathrm{E}-04$ \\
\hline $1.60 \mathrm{E}+01$ & 293.2 & $6.15 \mathrm{E}+16$ & $2.25 \mathrm{E}-04$ \\
\hline $1.70 \mathrm{E}+01$ & 292.73 & $1.29 \mathrm{E}+16$ & $4.74 \mathrm{E}-05$ \\
\hline $1.80 \mathrm{E}+01$ & 297.65 & $9.44 \mathrm{E}+15$ & $3.51 \mathrm{E}-05$ \\
\hline $1.90 \mathrm{E}+01$ & 307.26 & $5.59 \mathrm{E}+15$ & $2.15 \mathrm{E}-05$ \\
\hline $2.00 \mathrm{E}+01$ & 316.36 & $0.00 \mathrm{E}+00$ & $0.00 \mathrm{E}+00$ \\
\hline & & & $6.04 \mathrm{E}+00$ \\
\hline
\end{tabular}


Table 14. Calculated fluence and DPA for capsule 7 of the UCSB experiment.

\begin{tabular}{|c|c|c|c|c|c|c|c|}
\hline \multirow{2}{*}{$\begin{array}{c}\text { Upper } \\
\text { Energy(MeV) }\end{array}$} & \multirow{2}{*}{$\begin{array}{l}\text { DPA xsec } \\
\text { keV-barn }\end{array}$} & \multicolumn{2}{|c|}{$7-3$} & \multicolumn{2}{|c|}{$7-2$} & \multicolumn{2}{|c|}{$7-1$} \\
\hline & & Total Fluence & DPA & Total Fluence & DPA & Total Fluence & DPA \\
\hline $1.00 \mathrm{E}-10$ & 0 & $6.39 E+15$ & & $6.54 \mathrm{E}+15$ & & $5.41 \mathrm{E}+15$ & \\
\hline $1.00 \mathrm{E}-09$ & 7.73 & $8.31 \mathrm{E}+17$ & 8.03E-05 & $9.11 \mathrm{E}+17$ & $8.80 \mathrm{E}-05$ & $8.12 \mathrm{E}+17$ & $7.85 \mathrm{E}-05$ \\
\hline $1.00 \mathrm{E}-08$ & 2.444 & $1.24 \mathrm{E}+20$ & 3.77E-03 & $1.25 \mathrm{E}+20$ & $3.82 \mathrm{E}-03$ & $1.29 \mathrm{E}+20$ & 3.93E-03 \\
\hline $2.30 \mathrm{E}-08$ & 1.278 & $4.51 \mathrm{E}+20$ & $7.21 \mathrm{E}-03$ & $4.50 \mathrm{E}+20$ & $7.20 \mathrm{E}-03$ & $4.66 \mathrm{E}+20$ & $7.44 \mathrm{E}-03$ \\
\hline $5.00 \mathrm{E}-08$ & 0.857 & $1.11 \mathrm{E}+21$ & $1.19 \mathrm{E}-02$ & $1.13 \mathrm{E}+21$ & $1.21 \mathrm{E}-02$ & $1.15 \mathrm{E}+21$ & $1.24 \mathrm{E}-02$ \\
\hline $7.60 \mathrm{E}-08$ & 0.644 & $8.05 E+20$ & $6.48 \mathrm{E}-03$ & $8.08 \mathrm{E}+20$ & $6.51 \mathrm{E}-03$ & $8.25 \mathrm{E}+20$ & $6.64 \mathrm{E}-03$ \\
\hline $1.15 \mathrm{E}-07$ & 0.523 & $6.87 E+20$ & 4.49E-03 & $6.99 \mathrm{E}+20$ & 4.57E-03 & $7.04 \mathrm{E}+20$ & 4.60E-03 \\
\hline $1.70 \mathrm{E}-07$ & 0.428 & $4.24 \mathrm{E}+20$ & 2.27E-03 & $4.39 \mathrm{E}+20$ & $2.35 \mathrm{E}-03$ & $4.44 \mathrm{E}+20$ & 2.37E-03 \\
\hline $2.55 \mathrm{E}-07$ & 0.351 & $2.94 \mathrm{E}+20$ & $1.29 \mathrm{E}-03$ & $3.02 \mathrm{E}+20$ & $1.33 \mathrm{E}-03$ & $3.04 \mathrm{E}+20$ & $1.33 \mathrm{E}-03$ \\
\hline $3.80 \mathrm{E}-07$ & 0.287 & $2.46 \mathrm{E}+20$ & $8.83 \mathrm{E}-04$ & $2.50 \mathrm{E}+20$ & 8.97E-04 & $2.57 \mathrm{E}+20$ & $9.24 \mathrm{E}-04$ \\
\hline $5.50 \mathrm{E}-07$ & 0.237 & $2.23 \mathrm{E}+20$ & $6.62 \mathrm{E}-04$ & $2.25 \mathrm{E}+20$ & $6.67 \mathrm{E}-04$ & $2.33 \mathrm{E}+20$ & $6.91 \mathrm{E}-04$ \\
\hline $8.40 \mathrm{E}-07$ & 0.194 & $2.53 \mathrm{E}+20$ & $6.14 \mathrm{E}-04$ & $2.56 \mathrm{E}+20$ & $6.22 \mathrm{E}-04$ & $2.57 \mathrm{E}+20$ & $6.23 \mathrm{E}-04$ \\
\hline $1.28 \mathrm{E}-06$ & 0.157 & $2.43 \mathrm{E}+20$ & 4.76E-04 & $2.49 \mathrm{E}+20$ & $4.88 \mathrm{E}-04$ & $2.50 \mathrm{E}+20$ & 4.91E-04 \\
\hline $1.90 \mathrm{E}-06$ & 0.128 & $2.26 \mathrm{E}+20$ & $3.62 \mathrm{E}-04$ & $2.27 E+20$ & $3.64 \mathrm{E}-04$ & $2.32 \mathrm{E}+20$ & 3.71E-04 \\
\hline $2.80 \mathrm{E}-06$ & 0.105 & $2.14 \mathrm{E}+20$ & $2.80 \mathrm{E}-04$ & $2.18 \mathrm{E}+20$ & $2.86 \mathrm{E}-04$ & $2.22 \mathrm{E}+20$ & 2.91E-04 \\
\hline 4.25E-06 & 0.086 & $2.31 \mathrm{E}+20$ & $2.48 \mathrm{E}-04$ & $2.29 \mathrm{E}+20$ & $2.46 \mathrm{E}-04$ & $2.33 \mathrm{E}+20$ & $2.51 \mathrm{E}-04$ \\
\hline $6.30 \mathrm{E}-06$ & 0.07 & $2.15 E+20$ & $1.88 \mathrm{E}-04$ & $2.15 \mathrm{E}+20$ & $1.88 \mathrm{E}-04$ & $2.21 \mathrm{E}+20$ & $1.93 \mathrm{E}-04$ \\
\hline $9.20 \mathrm{E}-06$ & 0.058 & $2.12 \mathrm{E}+20$ & $1.53 \mathrm{E}-04$ & $2.09 \mathrm{E}+20$ & $1.52 \mathrm{E}-04$ & $2.11 \mathrm{E}+20$ & $1.53 \mathrm{E}-04$ \\
\hline $1.35 \mathrm{E}-05$ & 0.048 & $2.11 \mathrm{E}+20$ & $1.27 \mathrm{E}-04$ & $2.22 \mathrm{E}+20$ & $1.33 \mathrm{E}-04$ & $2.19 E+20$ & $1.32 \mathrm{E}-04$ \\
\hline $2.10 \mathrm{E}-05$ & 0.038 & $2.42 \mathrm{E}+20$ & $1.15 \mathrm{E}-04$ & $2.53 \mathrm{E}+20$ & $1.20 \mathrm{E}-04$ & $2.51 E+20$ & 1.19E-04 \\
\hline $3.00 \mathrm{E}-05$ & 0.031 & $2.02 \mathrm{E}+20$ & $7.82 \mathrm{E}-05$ & $1.99 \mathrm{E}+20$ & $7.72 \mathrm{E}-05$ & $2.07 E+20$ & 8.01E-05 \\
\hline $4.50 \mathrm{E}-05$ & 0.026 & $2.31 \mathrm{E}+20$ & 7.51E-05 & $2.36 \mathrm{E}+20$ & $7.66 \mathrm{E}-05$ & $2.39 \mathrm{E}+20$ & 7.76E-05 \\
\hline $6.90 \mathrm{E}-05$ & 0.021 & $2.46 \mathrm{E}+20$ & $6.45 \mathrm{E}-05$ & $2.50 \mathrm{E}+20$ & $6.56 \mathrm{E}-05$ & $2.55 \mathrm{E}+20$ & $6.69 \mathrm{E}-05$ \\
\hline $1.00 \mathrm{E}-04$ & 0.017 & $2.17 E+20$ & 4.62E-05 & $2.19 \mathrm{E}+20$ & 4.65E-05 & $2.26 \mathrm{E}+20$ & 4.81E-05 \\
\hline $1.35 \mathrm{E}-04$ & 0.014 & $1.79 E+20$ & $3.13 \mathrm{E}-05$ & $1.79 E+20$ & $3.13 E-05$ & $1.83 \mathrm{E}+20$ & $3.21 \mathrm{E}-05$ \\
\hline $1.70 \mathrm{E}-04$ & 0.014 & $1.38 \mathrm{E}+20$ & $2.41 \mathrm{E}-05$ & $1.41 \mathrm{E}+20$ & 2.47E-05 & $1.42 \mathrm{E}+20$ & $2.48 \mathrm{E}-05$ \\
\hline $2.20 \mathrm{E}-04$ & 0.011 & $1.55 \mathrm{E}+20$ & $2.13 \mathrm{E}-05$ & $1.57 \mathrm{E}+20$ & $2.16 \mathrm{E}-05$ & $1.58 \mathrm{E}+20$ & 2.17E-05 \\
\hline
\end{tabular}




\begin{tabular}{|c|c|c|c|c|c|c|c|}
\hline \multirow{2}{*}{$\begin{array}{c}\text { Upper } \\
\text { Energy(MeV) }\end{array}$} & \multirow{2}{*}{$\begin{array}{l}\text { DPA xsec } \\
\text { keV-barn }\end{array}$} & \multicolumn{2}{|c|}{$7-3$} & \multicolumn{2}{|c|}{$7-2$} & \multicolumn{2}{|c|}{$7-1$} \\
\hline & & Total Fluence & DPA & Total Fluence & DPA & Total Fluence & DPA \\
\hline $2.80 \mathrm{E}-04$ & 0.011 & $1.49 \mathrm{E}+20$ & $2.05 \mathrm{E}-05$ & $1.44 \mathrm{E}+20$ & $1.97 \mathrm{E}-05$ & $1.52 \mathrm{E}+20$ & $2.09 \mathrm{E}-05$ \\
\hline $3.60 \mathrm{E}-04$ & 0.01 & $1.48 \mathrm{E}+20$ & $1.85 \mathrm{E}-05$ & $1.47 \mathrm{E}+20$ & $1.83 \mathrm{E}-05$ & $1.53 \mathrm{E}+20$ & $1.92 \mathrm{E}-05$ \\
\hline $4.50 \mathrm{E}-04$ & 0.007 & $1.31 \mathrm{E}+20$ & $1.15 \mathrm{E}-05$ & $1.28 \mathrm{E}+20$ & $1.12 \mathrm{E}-05$ & $1.32 \mathrm{E}+20$ & $1.16 \mathrm{E}-05$ \\
\hline $5.75 \mathrm{E}-04$ & 0.005 & $1.49 \mathrm{E}+20$ & $9.31 \mathrm{E}-06$ & $1.49 \mathrm{E}+20$ & $9.31 \mathrm{E}-06$ & $1.52 \mathrm{E}+20$ & $9.52 \mathrm{E}-06$ \\
\hline $7.60 \mathrm{E}-04$ & 0.129 & $1.69 \mathrm{E}+20$ & 2.73E-04 & $1.75 \mathrm{E}+20$ & $2.82 \mathrm{E}-04$ & $1.73 \mathrm{E}+20$ & $2.79 \mathrm{E}-04$ \\
\hline $9.60 \mathrm{E}-04$ & 0.308 & $1.48 \mathrm{E}+20$ & 5.69E-04 & $1.47 \mathrm{E}+20$ & $5.66 \mathrm{E}-04$ & $1.45 \mathrm{E}+20$ & 5.59E-04 \\
\hline $1.28 \mathrm{E}-03$ & 0.672 & $1.73 \mathrm{E}+20$ & $1.45 \mathrm{E}-03$ & $1.78 \mathrm{E}+20$ & $1.49 \mathrm{E}-03$ & $1.76 \mathrm{E}+20$ & $1.48 \mathrm{E}-03$ \\
\hline $1.60 \mathrm{E}-03$ & 0.477 & $1.36 \mathrm{E}+20$ & 8.10E-04 & $1.35 \mathrm{E}+20$ & 8.04E-04 & $1.40 \mathrm{E}+20$ & 8.36E-04 \\
\hline $2.00 \mathrm{E}-03$ & 0.511 & $1.42 \mathrm{E}+20$ & 9.07E-04 & $1.41 \mathrm{E}+20$ & $9.03 \mathrm{E}-04$ & $1.42 \mathrm{E}+20$ & $9.05 \mathrm{E}-04$ \\
\hline $2.70 \mathrm{E}-03$ & 0.568 & $1.80 \mathrm{E}+20$ & $1.28 \mathrm{E}-03$ & $1.83 \mathrm{E}+20$ & $1.30 \mathrm{E}-03$ & $1.88 \mathrm{E}+20$ & $1.34 \mathrm{E}-03$ \\
\hline $3.40 \mathrm{E}-03$ & 0.632 & $1.49 \mathrm{E}+20$ & $1.18 \mathrm{E}-03$ & $1.47 \mathrm{E}+20$ & $1.16 \mathrm{E}-03$ & $1.50 \mathrm{E}+20$ & 1.19E-03 \\
\hline $4.50 \mathrm{E}-03$ & 0.822 & $1.74 \mathrm{E}+20$ & $1.79 \mathrm{E}-03$ & $1.85 \mathrm{E}+20$ & $1.90 \mathrm{E}-03$ & $1.80 \mathrm{E}+20$ & $1.85 \mathrm{E}-03$ \\
\hline $5.50 \mathrm{E}-03$ & 0.826 & $1.29 \mathrm{E}+20$ & $1.34 \mathrm{E}-03$ & $1.35 \mathrm{E}+20$ & $1.39 \mathrm{E}-03$ & $1.37 \mathrm{E}+20$ & $1.41 \mathrm{E}-03$ \\
\hline 7.20E-03 & 1.671 & $1.73 \mathrm{E}+20$ & 3.62E-03 & $1.85 \mathrm{E}+20$ & $3.86 \mathrm{E}-03$ & $1.82 \mathrm{E}+20$ & $3.80 \mathrm{E}-03$ \\
\hline $9.20 \mathrm{E}-03$ & 3.709 & $1.39 \mathrm{E}+20$ & $6.45 \mathrm{E}-03$ & $1.42 \mathrm{E}+20$ & $6.56 \mathrm{E}-03$ & $1.46 \mathrm{E}+20$ & $6.78 \mathrm{E}-03$ \\
\hline $1.20 \mathrm{E}-02$ & 1.491 & $1.71 \mathrm{E}+20$ & $3.18 \mathrm{E}-03$ & $1.68 \mathrm{E}+20$ & $3.14 \mathrm{E}-03$ & $1.80 \mathrm{E}+20$ & $3.35 \mathrm{E}-03$ \\
\hline $1.50 \mathrm{E}-02$ & 1.156 & $1.53 \mathrm{E}+20$ & $2.21 \mathrm{E}-03$ & $1.55 \mathrm{E}+20$ & $2.24 \mathrm{E}-03$ & $1.54 \mathrm{E}+20$ & $2.23 \mathrm{E}-03$ \\
\hline $1.90 \mathrm{E}-02$ & 0.973 & $1.62 \mathrm{E}+20$ & 1.97E-03 & $1.65 \mathrm{E}+20$ & $2.01 \mathrm{E}-03$ & $1.59 \mathrm{E}+20$ & $1.93 \mathrm{E}-03$ \\
\hline $2.55 \mathrm{E}-02$ & 0.555 & $2.30 \mathrm{E}+20$ & $1.60 \mathrm{E}-03$ & $2.31 \mathrm{E}+20$ & $1.60 \mathrm{E}-03$ & $2.37 \mathrm{E}+20$ & $1.64 \mathrm{E}-03$ \\
\hline $3.20 \mathrm{E}-02$ & 26.762 & $2.06 \mathrm{E}+20$ & $6.90 \mathrm{E}-02$ & $2.14 \mathrm{E}+20$ & 7.16E-02 & $2.12 \mathrm{E}+20$ & 7.10E-02 \\
\hline $4.00 \mathrm{E}-02$ & 8.161 & $9.74 \mathrm{E}+19$ & $9.94 \mathrm{E}-03$ & $9.92 \mathrm{E}+19$ & $1.01 \mathrm{E}-02$ & $1.02 \mathrm{E}+20$ & $1.04 \mathrm{E}-02$ \\
\hline $5.25 \mathrm{E}-02$ & 6.515 & $2.02 \mathrm{E}+20$ & $1.65 \mathrm{E}-02$ & $2.06 \mathrm{E}+20$ & $1.68 \mathrm{E}-02$ & $2.07 E+20$ & $1.69 \mathrm{E}-02$ \\
\hline $6.60 \mathrm{E}-02$ & 6.576 & $1.97 \mathrm{E}+20$ & $1.62 \mathrm{E}-02$ & $1.96 \mathrm{E}+20$ & $1.61 \mathrm{E}-02$ & $2.05 \mathrm{E}+20$ & $1.69 \mathrm{E}-02$ \\
\hline $8.80 \mathrm{E}-02$ & 13.137 & $2.99 \mathrm{E}+20$ & 4.91E-02 & $3.01 E+20$ & 4.93E-02 & $3.04 \mathrm{E}+20$ & $5.00 \mathrm{E}-02$ \\
\hline 1.10E-01 & 11.84 & $1.77 \mathrm{E}+20$ & $2.62 \mathrm{E}-02$ & $1.86 \mathrm{E}+20$ & $2.75 \mathrm{E}-02$ & $1.87 \mathrm{E}+20$ & 2.77E-02 \\
\hline $1.35 \mathrm{E}-01$ & 11.27 & $2.67 E+20$ & 3.76E-02 & $2.69 \mathrm{E}+20$ & $3.79 \mathrm{E}-02$ & $2.76 \mathrm{E}+20$ & $3.88 \mathrm{E}-02$ \\
\hline $1.60 \mathrm{E}-01$ & 21.53 & $1.60 \mathrm{E}+20$ & 4.30E-02 & $1.56 \mathrm{E}+20$ & $4.21 \mathrm{E}-02$ & $1.63 \mathrm{E}+20$ & 4.39E-02 \\
\hline $1.90 \mathrm{E}-01$ & 15.85 & $2.18 \mathrm{E}+20$ & 4.33E-02 & $2.21 \mathrm{E}+20$ & $4.38 \mathrm{E}-02$ & $2.25 \mathrm{E}+20$ & 4.45E-02 \\
\hline $2.20 \mathrm{E}-01$ & 22.01 & $1.73 \mathrm{E}+20$ & 4.76E-02 & $1.83 \mathrm{E}+20$ & $5.02 \mathrm{E}-02$ & $1.82 \mathrm{E}+20$ & $5.02 \mathrm{E}-02$ \\
\hline
\end{tabular}




\begin{tabular}{|c|c|c|c|c|c|c|c|}
\hline \multirow{2}{*}{$\begin{array}{c}\text { Upper } \\
\text { Energy(MeV) }\end{array}$} & \multirow{2}{*}{$\begin{array}{l}\text { DPA xsec } \\
\text { keV-barn }\end{array}$} & \multicolumn{2}{|c|}{$7-3$} & \multicolumn{2}{|c|}{$7-2$} & \multicolumn{2}{|c|}{$7-1$} \\
\hline & & Total Fluence & DPA & Total Fluence & DPA & Total Fluence & DPA \\
\hline $2.55 \mathrm{E}-01$ & 18.27 & $2.09 \mathrm{E}+20$ & 4.77E-02 & $2.01 E+20$ & 4.59E-02 & $2.11 E+20$ & $4.81 \mathrm{E}-02$ \\
\hline $2.90 \mathrm{E}-01$ & 17.37 & $2.19 E+20$ & 4.76E-02 & $2.24 \mathrm{E}+20$ & $4.85 \mathrm{E}-02$ & $2.30 E+20$ & $5.00 \mathrm{E}-02$ \\
\hline $3.20 \mathrm{E}-01$ & 13.51 & $1.59 \mathrm{E}+20$ & $2.68 \mathrm{E}-02$ & $1.57 \mathrm{E}+20$ & $2.64 \mathrm{E}-02$ & $1.59 \mathrm{E}+20$ & $2.69 \mathrm{E}-02$ \\
\hline $3.60 \mathrm{E}-01$ & 25.14 & $2.21 \mathrm{E}+20$ & $6.96 \mathrm{E}-02$ & $2.24 \mathrm{E}+20$ & 7.03E-02 & $2.26 \mathrm{E}+20$ & 7.12E-02 \\
\hline 4.00E-01 & 46.52 & $1.91 \mathrm{E}+20$ & $1.11 \mathrm{E}-01$ & $1.96 \mathrm{E}+20$ & 1.14E-01 & $1.96 \mathrm{E}+20$ & $1.14 \mathrm{E}-01$ \\
\hline $4.50 \mathrm{E}-01$ & 43.04 & $1.79 \mathrm{E}+20$ & $9.65 \mathrm{E}-02$ & $1.81 \mathrm{E}+20$ & $9.73 \mathrm{E}-02$ & $1.86 \mathrm{E}+20$ & $1.00 \mathrm{E}-01$ \\
\hline $5.00 \mathrm{E}-01$ & 36.4 & $2.01 \mathrm{E}+20$ & $9.15 \mathrm{E}-02$ & $2.05 E+20$ & $9.34 \mathrm{E}-02$ & $2.11 \mathrm{E}+20$ & $9.60 \mathrm{E}-02$ \\
\hline $5.50 \mathrm{E}-01$ & 33.22 & $2.04 \mathrm{E}+20$ & $8.46 \mathrm{E}-02$ & $2.01 E+20$ & $8.35 \mathrm{E}-02$ & $2.07 E+20$ & $8.60 \mathrm{E}-02$ \\
\hline $6.00 \mathrm{E}-01$ & 34.18 & $1.90 \mathrm{E}+20$ & 8.13E-02 & $1.96 \mathrm{E}+20$ & $8.38 \mathrm{E}-02$ & $1.99 \mathrm{E}+20$ & $8.52 \mathrm{E}-02$ \\
\hline $6.60 \mathrm{E}-01$ & 19.64 & $2.39 \mathrm{E}+20$ & 5.87E-02 & $2.37 E+20$ & $5.81 \mathrm{E}-02$ & $2.46 \mathrm{E}+20$ & $6.03 E-02$ \\
\hline 7.20E-01 & 48.89 & $2.46 \mathrm{E}+20$ & $1.50 \mathrm{E}-01$ & $2.50 \mathrm{E}+20$ & $1.53 \mathrm{E}-01$ & $2.58 \mathrm{E}+20$ & $1.58 \mathrm{E}-01$ \\
\hline $7.80 \mathrm{E}-01$ & 74.22 & $2.31 E+20$ & $2.14 \mathrm{E}-01$ & $2.34 \mathrm{E}+20$ & 2.17E-01 & $2.38 \mathrm{E}+20$ & $2.21 \mathrm{E}-01$ \\
\hline $8.40 \mathrm{E}-01$ & 44.02 & $1.81 \mathrm{E}+20$ & $9.98 \mathrm{E}-02$ & $1.90 \mathrm{E}+20$ & $1.04 \mathrm{E}-01$ & $1.90 \mathrm{E}+20$ & $1.04 \mathrm{E}-01$ \\
\hline $9.20 \mathrm{E}-01$ & 40.97 & $2.21 \mathrm{E}+20$ & 1.13E-01 & $2.22 \mathrm{E}+20$ & $1.14 \mathrm{E}-01$ & $2.24 \mathrm{E}+20$ & $1.15 \mathrm{E}-01$ \\
\hline $1.00 \mathrm{E}+00$ & 50.95 & $2.02 E+20$ & 1.29E-01 & $1.96 \mathrm{E}+20$ & $1.25 \mathrm{E}-01$ & $2.10 \mathrm{E}+20$ & $1.34 \mathrm{E}-01$ \\
\hline $1.20 \mathrm{E}+00$ & 50.09 & $4.76 \mathrm{E}+20$ & $2.98 \mathrm{E}-01$ & $4.79 \mathrm{E}+20$ & $3.00 \mathrm{E}-01$ & $4.87 \mathrm{E}+20$ & 3.05E-01 \\
\hline $1.40 \mathrm{E}+00$ & 64.54 & $4.30 \mathrm{E}+20$ & 3.47E-01 & $4.32 \mathrm{E}+20$ & $3.48 \mathrm{E}-01$ & $4.36 \mathrm{E}+20$ & $3.52 \mathrm{E}-01$ \\
\hline $1.60 \mathrm{E}+00$ & 73.58 & $3.61 \mathrm{E}+20$ & $3.32 \mathrm{E}-01$ & $3.66 \mathrm{E}+20$ & 3.37E-01 & $3.70 \mathrm{E}+20$ & $3.41 \mathrm{E}-01$ \\
\hline $1.80 \mathrm{E}+00$ & 76.46 & $3.19 \mathrm{E}+20$ & 3.05E-01 & $3.20 \mathrm{E}+20$ & $3.06 \mathrm{E}-01$ & $3.25 \mathrm{E}+20$ & 3.11E-01 \\
\hline $2.00 \mathrm{E}+00$ & 95.15 & $2.78 \mathrm{E}+20$ & 3.31E-01 & $2.83 E+20$ & 3.36E-01 & $2.89 \mathrm{E}+20$ & $3.43 \mathrm{E}-01$ \\
\hline $2.30 \mathrm{E}+00$ & 93.75 & $3.53 \mathrm{E}+20$ & 4.13E-01 & $3.55 \mathrm{E}+20$ & 4.16E-01 & $3.51 \mathrm{E}+20$ & 4.11E-01 \\
\hline $2.60 \mathrm{E}+00$ & 112.05 & $3.19 E+20$ & 4.47E-01 & $3.26 \mathrm{E}+20$ & 4.57E-01 & $3.30 \mathrm{E}+20$ & $4.62 \mathrm{E}-01$ \\
\hline $2.90 \mathrm{E}+00$ & 123.55 & $2.40 \mathrm{E}+20$ & $3.71 \mathrm{E}-01$ & $2.42 \mathrm{E}+20$ & $3.74 \mathrm{E}-01$ & $2.49 \mathrm{E}+20$ & $3.85 \mathrm{E}-01$ \\
\hline $3.30 \mathrm{E}+00$ & 133.45 & $2.42 \mathrm{E}+20$ & 4.03E-01 & $2.36 \mathrm{E}+20$ & 3.94E-01 & $2.48 \mathrm{E}+20$ & 4.14E-01 \\
\hline $3.70 \mathrm{E}+00$ & 135.25 & $1.61 \mathrm{E}+20$ & 2.72E-01 & $1.70 \mathrm{E}+20$ & 2.87E-01 & $1.66 \mathrm{E}+20$ & $2.80 \mathrm{E}-01$ \\
\hline $4.10 \mathrm{E}+00$ & 149.55 & $1.28 \mathrm{E}+20$ & 2.39E-01 & $1.28 \mathrm{E}+20$ & $2.39 \mathrm{E}-01$ & $1.29 \mathrm{E}+20$ & $2.41 \mathrm{E}-01$ \\
\hline $4.50 \mathrm{E}+00$ & 158.25 & $1.00 \mathrm{E}+20$ & $1.99 \mathrm{E}-01$ & $9.77 \mathrm{E}+19$ & $1.93 \mathrm{E}-01$ & $1.01 \mathrm{E}+20$ & $1.99 \mathrm{E}-01$ \\
\hline $5.00 \mathrm{E}+00$ & 168.55 & $9.29 \mathrm{E}+19$ & 1.96E-01 & $9.71 \mathrm{E}+19$ & $2.04 \mathrm{E}-01$ & $9.62 \mathrm{E}+19$ & $2.03 \mathrm{E}-01$ \\
\hline $5.50 \mathrm{E}+00$ & 176.46 & $6.48 \mathrm{E}+19$ & $1.43 \mathrm{E}-01$ & $6.39 E+19$ & $1.41 \mathrm{E}-01$ & $6.40 E+19$ & $1.41 \mathrm{E}-01$ \\
\hline
\end{tabular}




\begin{tabular}{|c|c|c|c|c|c|c|c|}
\hline \multirow{2}{*}{$\begin{array}{c}\text { Upper } \\
\text { Energy(MeV) }\end{array}$} & \multirow{2}{*}{$\begin{array}{l}\text { DPA xsec } \\
\text { keV-barn }\end{array}$} & \multicolumn{2}{|c|}{$7-3$} & \multicolumn{2}{|c|}{$7-2$} & \multicolumn{2}{|c|}{$7-1$} \\
\hline & & Total Fluence & DPA & Total Fluence & DPA & Total Fluence & DPA \\
\hline $6.00 \mathrm{E}+00$ & 183.06 & $4.30 E+19$ & $9.85 \mathrm{E}-02$ & 4.17E+19 & $9.54 \mathrm{E}-02$ & $4.55 E+19$ & $1.04 \mathrm{E}-01$ \\
\hline $6.70 \mathrm{E}+00$ & 189.26 & $3.95 \mathrm{E}+19$ & $9.35 \mathrm{E}-02$ & $4.00 E+19$ & $9.45 \mathrm{E}-02$ & $4.14 \mathrm{E}+19$ & $9.79 \mathrm{E}-02$ \\
\hline $7.40 E+00$ & 196.67 & $2.39 E+19$ & 5.87E-02 & $2.32 E+19$ & $5.71 \mathrm{E}-02$ & $2.41 E+19$ & $5.92 \mathrm{E}-02$ \\
\hline $8.20 \mathrm{E}+00$ & 203.37 & $1.60 \mathrm{E}+19$ & 4.07E-02 & $1.56 \mathrm{E}+19$ & $3.96 \mathrm{E}-02$ & $1.55 \mathrm{E}+19$ & 3.93E-02 \\
\hline $9.00 \mathrm{E}+00$ & 214.58 & $7.93 \mathrm{E}+18$ & $2.13 \mathrm{E}-02$ & $9.20 \mathrm{E}+18$ & $2.47 \mathrm{E}-02$ & $8.84 \mathrm{E}+18$ & $2.37 \mathrm{E}-02$ \\
\hline $1.00 \mathrm{E}+01$ & 225.69 & $5.62 E+18$ & $1.59 \mathrm{E}-02$ & $5.13 E+18$ & $1.45 \mathrm{E}-02$ & $5.41 \mathrm{E}+18$ & $1.53 \mathrm{E}-02$ \\
\hline $1.10 \mathrm{E}+01$ & 237.6 & $2.67 \mathrm{E}+18$ & 7.92E-03 & $1.97 \mathrm{E}+18$ & $5.85 \mathrm{E}-03$ & $2.30 \mathrm{E}+18$ & $6.83 \mathrm{E}-03$ \\
\hline $1.20 \mathrm{E}+01$ & 247.41 & $1.03 \mathrm{E}+18$ & 3.19E-03 & $9.51 E+17$ & $2.94 \mathrm{E}-03$ & $1.24 \mathrm{E}+18$ & $3.84 \mathrm{E}-03$ \\
\hline $1.30 \mathrm{E}+01$ & 258.52 & $3.66 \mathrm{E}+17$ & $1.18 \mathrm{E}-03$ & $3.31 \mathrm{E}+17$ & $1.07 \mathrm{E}-03$ & $4.74 \mathrm{E}+17$ & $1.53 \mathrm{E}-03$ \\
\hline $1.40 \mathrm{E}+01$ & 271.35 & $1.09 \mathrm{E}+17$ & 3.70E-04 & $1.86 \mathrm{E}+17$ & $6.29 \mathrm{E}-04$ & $1.51 \mathrm{E}+17$ & $5.12 \mathrm{E}-04$ \\
\hline $1.50 \mathrm{E}+01$ & 290.27 & $5.29 \mathrm{E}+16$ & $1.92 \mathrm{E}-04$ & $1.74 \mathrm{E}+17$ & $6.32 \mathrm{E}-04$ & $5.44 \mathrm{E}+16$ & 1.97E-04 \\
\hline $1.60 \mathrm{E}+01$ & 293.2 & $1.19 \mathrm{E}+17$ & 4.37E-04 & $1.49 \mathrm{E}+17$ & $5.48 \mathrm{E}-04$ & $3.13 E+16$ & $1.15 \mathrm{E}-04$ \\
\hline $1.70 \mathrm{E}+01$ & 292.73 & $7.04 \mathrm{E}+16$ & 2.57E-04 & $5.28 \mathrm{E}+16$ & $1.93 \mathrm{E}-04$ & $7.36 \mathrm{E}+15$ & 2.69E-05 \\
\hline $1.80 \mathrm{E}+01$ & 297.65 & $3.43 E+16$ & $1.28 \mathrm{E}-04$ & $0.00 \mathrm{E}+00$ & $0.00 \mathrm{E}+00$ & $0.00 \mathrm{E}+00$ & $0.00 \mathrm{E}+0 \mathrm{C}$ \\
\hline $1.90 \mathrm{E}+01$ & 307.26 & $0.00 E+00$ & $0.00 \mathrm{E}+00$ & $0.00 E+00$ & $0.00 \mathrm{E}+00$ & $0.00 E+00$ & $0.00 \mathrm{E}+0 \mathrm{C}$ \\
\hline \multirow[t]{2}{*}{$2.00 \mathrm{E}+01$} & 316.36 & $0.00 \mathrm{E}+00$ & $0.00 \mathrm{E}+00$ & $0.00 \mathrm{E}+00$ & $0.00 \mathrm{E}+00$ & $0.00 \mathrm{E}+00$ & $0.00 \mathrm{E}+0 \mathrm{C}$ \\
\hline & & & $6.49 E+00$ & & $6.54 E+00$ & & $6.65 E+0 C$ \\
\hline
\end{tabular}


Table 15. Calculated fluence and DPA for capsule 6 of the UCSB experiment.

\begin{tabular}{|c|c|c|c|c|c|}
\hline \multirow{2}{*}{$\begin{array}{c}\text { Upper } \\
\text { Energy(MeV) }\end{array}$} & \multirow{2}{*}{$\begin{array}{l}\text { DPA xsec } \\
\text { keV-barn }\end{array}$} & \multicolumn{2}{|c|}{$6 A$} & \multicolumn{2}{|c|}{ 6-B } \\
\hline & & Total Fluence & DPA & Total Fluence & DPA \\
\hline $1.00 \mathrm{E}-10$ & 0 & $3.90 \mathrm{E}+14$ & & $1.06 \mathrm{E}+15$ & \\
\hline $1.00 \mathrm{E}-09$ & 7.73 & $2.32 \mathrm{E}+17$ & $2.24 \mathrm{E}-05$ & $6.29 \mathrm{E}+17$ & $6.08 \mathrm{E}-05$ \\
\hline $1.00 \mathrm{E}-08$ & 2.444 & $3.48 \mathrm{E}+19$ & $1.06 \mathrm{E}-03$ & $9.44 \mathrm{E}+19$ & $2.88 \mathrm{E}-03$ \\
\hline $2.30 \mathrm{E}-08$ & 1.278 & $1.27 \mathrm{E}+20$ & $2.03 \mathrm{E}-03$ & $3.44 \mathrm{E}+20$ & 5.49E-03 \\
\hline $5.00 \mathrm{E}-08$ & 0.857 & $3.15 E+20$ & 3.37E-03 & $8.53 E+20$ & $9.13 \mathrm{E}-03$ \\
\hline $7.60 \mathrm{E}-08$ & 0.644 & $2.21 \mathrm{E}+20$ & $1.78 \mathrm{E}-03$ & $5.99 \mathrm{E}+20$ & $4.82 \mathrm{E}-03$ \\
\hline $1.15 \mathrm{E}-07$ & 0.523 & $1.91 \mathrm{E}+20$ & $1.25 \mathrm{E}-03$ & $5.19 E+20$ & 3.39E-03 \\
\hline $1.70 \mathrm{E}-07$ & 0.428 & $1.19 \mathrm{E}+20$ & $6.37 \mathrm{E}-04$ & $3.23 \mathrm{E}+20$ & $1.73 \mathrm{E}-03$ \\
\hline $2.55 \mathrm{E}-07$ & 0.351 & $8.27 \mathrm{E}+19$ & $3.63 \mathrm{E}-04$ & $2.24 \mathrm{E}+20$ & $9.84 \mathrm{E}-04$ \\
\hline $3.80 \mathrm{E}-07$ & 0.287 & $6.92 \mathrm{E}+19$ & $2.48 \mathrm{E}-04$ & $1.87 \mathrm{E}+20$ & $6.73 \mathrm{E}-04$ \\
\hline $5.50 \mathrm{E}-07$ & 0.237 & $6.31 E+19$ & $1.87 \mathrm{E}-04$ & $1.71 \mathrm{E}+20$ & 5.07E-04 \\
\hline $8.40 \mathrm{E}-07$ & 0.194 & $6.99 \mathrm{E}+19$ & $1.70 \mathrm{E}-04$ & $1.90 \mathrm{E}+20$ & 4.60E-04 \\
\hline $1.28 \mathrm{E}-06$ & 0.157 & $6.83 E+19$ & $1.34 \mathrm{E}-04$ & $1.85 \mathrm{E}+20$ & 3.63E-04 \\
\hline $1.90 \mathrm{E}-06$ & 0.128 & $6.30 \mathrm{E}+19$ & $1.01 \mathrm{E}-04$ & $1.71 E+20$ & $2.73 \mathrm{E}-04$ \\
\hline $2.80 \mathrm{E}-06$ & 0.105 & $6.11 E+19$ & $8.02 \mathrm{E}-05$ & $1.66 \mathrm{E}+20$ & 2.17E-04 \\
\hline 4.25E-06 & 0.086 & $6.33 E+19$ & $6.80 \mathrm{E}-05$ & $1.72 \mathrm{E}+20$ & $1.84 \mathrm{E}-04$ \\
\hline $6.30 \mathrm{E}-06$ & 0.07 & $5.99 \mathrm{E}+19$ & $5.24 \mathrm{E}-05$ & $1.62 \mathrm{E}+20$ & $1.42 \mathrm{E}-04$ \\
\hline $9.20 \mathrm{E}-06$ & 0.058 & $5.89 \mathrm{E}+19$ & 4.27E-05 & $1.60 \mathrm{E}+20$ & 1.16E-04 \\
\hline $1.35 \mathrm{E}-05$ & 0.048 & $5.95 \mathrm{E}+19$ & 3.57E-05 & $1.61 \mathrm{E}+20$ & $9.68 \mathrm{E}-05$ \\
\hline $2.10 \mathrm{E}-05$ & 0.038 & $6.96 \mathrm{E}+19$ & 3.31E-05 & $1.89 \mathrm{E}+20$ & 8.96E-05 \\
\hline $3.00 \mathrm{E}-05$ & 0.031 & $5.73 E+19$ & $2.22 \mathrm{E}-05$ & $1.55 \mathrm{E}+20$ & $6.02 \mathrm{E}-05$ \\
\hline $4.50 \mathrm{E}-05$ & 0.026 & $6.57 E+19$ & $2.14 \mathrm{E}-05$ & $1.78 \mathrm{E}+20$ & 5.79E-05 \\
\hline $6.90 \mathrm{E}-05$ & 0.021 & $7.00 \mathrm{E}+19$ & $1.84 \mathrm{E}-05$ & $1.90 \mathrm{E}+20$ & 4.98E-05 \\
\hline $1.00 \mathrm{E}-04$ & 0.017 & $6.17 \mathrm{E}+19$ & $1.31 \mathrm{E}-05$ & $1.67 \mathrm{E}+20$ & 3.55E-05 \\
\hline $1.35 \mathrm{E}-04$ & 0.014 & $5.02 E+19$ & $8.78 \mathrm{E}-06$ & $1.36 \mathrm{E}+20$ & $2.38 \mathrm{E}-05$ \\
\hline $1.70 \mathrm{E}-04$ & 0.014 & $3.87 E+19$ & 6.77E-06 & $1.05 E+20$ & $1.84 \mathrm{E}-05$ \\
\hline $2.20 \mathrm{E}-04$ & 0.011 & $4.28 \mathrm{E}+19$ & $5.88 \mathrm{E}-06$ & $1.16 \mathrm{E}+20$ & $1.60 \mathrm{E}-05$ \\
\hline
\end{tabular}




\begin{tabular}{|c|c|c|c|c|c|}
\hline \multirow{2}{*}{$\begin{array}{c}\text { Upper } \\
\text { Energy(MeV) }\end{array}$} & \multirow{2}{*}{$\begin{array}{l}\text { DPA xsec } \\
\text { keV-barn }\end{array}$} & \multicolumn{2}{|c|}{$6 A$} & \multicolumn{2}{|c|}{ 6-B } \\
\hline & & Total Fluence & DPA & Total Fluence & DPA \\
\hline $2.80 \mathrm{E}-04$ & 0.011 & $4.14 \mathrm{E}+19$ & $5.69 \mathrm{E}-06$ & $1.12 \mathrm{E}+20$ & $1.54 \mathrm{E}-05$ \\
\hline $3.60 \mathrm{E}-04$ & 0.01 & $4.12 E+19$ & $5.15 \mathrm{E}-06$ & $1.12 \mathrm{E}+20$ & $1.40 \mathrm{E}-05$ \\
\hline 4.50E-04 & 0.007 & $3.64 \mathrm{E}+19$ & $3.18 \mathrm{E}-06$ & $9.85 \mathrm{E}+19$ & 8.62E-06 \\
\hline $5.75 \mathrm{E}-04$ & 0.005 & $4.12 E+19$ & 2.57E-06 & $1.12 \mathrm{E}+20$ & $6.98 \mathrm{E}-06$ \\
\hline $7.60 \mathrm{E}-04$ & 0.129 & $4.71 E+19$ & 7.59E-05 & $1.28 \mathrm{E}+20$ & 2.06E-04 \\
\hline $9.60 \mathrm{E}-04$ & 0.308 & $3.96 \mathrm{E}+19$ & $1.52 \mathrm{E}-04$ & $1.07 \mathrm{E}+20$ & 4.13E-04 \\
\hline $1.28 \mathrm{E}-03$ & 0.672 & $4.82 E+19$ & $4.05 \mathrm{E}-04$ & $1.31 \mathrm{E}+20$ & 1.10E-03 \\
\hline $1.60 \mathrm{E}-03$ & 0.477 & $3.82 E+19$ & $2.28 \mathrm{E}-04$ & $1.03 \mathrm{E}+20$ & 6.17E-04 \\
\hline $2.00 \mathrm{E}-03$ & 0.511 & $3.97 \mathrm{E}+19$ & $2.53 \mathrm{E}-04$ & $1.08 \mathrm{E}+20$ & 6.87E-04 \\
\hline $2.70 \mathrm{E}-03$ & 0.568 & $5.22 E+19$ & $3.70 \mathrm{E}-04$ & $1.41 E+20$ & $1.00 \mathrm{E}-03$ \\
\hline $3.40 \mathrm{E}-03$ & 0.632 & $4.19 E+19$ & $3.31 \mathrm{E}-04$ & $1.14 \mathrm{E}+20$ & $8.98 \mathrm{E}-04$ \\
\hline $4.50 \mathrm{E}-03$ & 0.822 & $4.99 E+19$ & $5.13 \mathrm{E}-04$ & $1.35 \mathrm{E}+20$ & $1.39 \mathrm{E}-03$ \\
\hline $5.50 \mathrm{E}-03$ & 0.826 & $3.71 E+19$ & 3.83E-04 & $1.01 \mathrm{E}+20$ & $1.04 \mathrm{E}-03$ \\
\hline 7.20E-03 & 1.671 & $4.90 \mathrm{E}+19$ & $1.02 \mathrm{E}-03$ & $1.33 \mathrm{E}+20$ & $2.78 \mathrm{E}-03$ \\
\hline $9.20 \mathrm{E}-03$ & 3.709 & $3.97 \mathrm{E}+19$ & $1.84 \mathrm{E}-03$ & $1.08 \mathrm{E}+20$ & 4.99E-03 \\
\hline $1.20 \mathrm{E}-02$ & 1.491 & $4.93 E+19$ & $9.19 \mathrm{E}-04$ & $1.34 \mathrm{E}+20$ & $2.49 \mathrm{E}-03$ \\
\hline $1.50 \mathrm{E}-02$ & 1.156 & $4.16 \mathrm{E}+19$ & $6.01 \mathrm{E}-04$ & $1.13 \mathrm{E}+20$ & $1.63 \mathrm{E}-03$ \\
\hline $1.90 \mathrm{E}-02$ & 0.973 & $4.41 \mathrm{E}+19$ & 5.37E-04 & $1.20 \mathrm{E}+20$ & $1.45 \mathrm{E}-03$ \\
\hline $2.55 \mathrm{E}-02$ & 0.555 & $6.34 \mathrm{E}+19$ & $4.40 \mathrm{E}-04$ & $1.72 \mathrm{E}+20$ & 1.19E-03 \\
\hline $3.20 \mathrm{E}-02$ & 26.762 & $5.79 E+19$ & $1.94 \mathrm{E}-02$ & $1.57 \mathrm{E}+20$ & $5.25 \mathrm{E}-02$ \\
\hline 4.00E-02 & 8.161 & $2.86 \mathrm{E}+19$ & $2.92 \mathrm{E}-03$ & $7.75 E+19$ & 7.91E-03 \\
\hline $5.25 \mathrm{E}-02$ & 6.515 & $5.62 \mathrm{E}+19$ & $4.58 \mathrm{E}-03$ & $1.52 \mathrm{E}+20$ & $1.24 \mathrm{E}-02$ \\
\hline $6.60 \mathrm{E}-02$ & 6.576 & $5.61 \mathrm{E}+19$ & 4.61E-03 & $1.52 \mathrm{E}+20$ & 1.25E-02 \\
\hline $8.80 \mathrm{E}-02$ & 13.137 & $8.10 E+19$ & $1.33 \mathrm{E}-02$ & $2.19 E+20$ & 3.60E-02 \\
\hline $1.10 \mathrm{E}-01$ & 11.84 & $5.16 \mathrm{E}+19$ & $7.64 \mathrm{E}-03$ & $1.40 \mathrm{E}+20$ & 2.07E-02 \\
\hline $1.35 \mathrm{E}-01$ & 11.27 & $7.53 E+19$ & $1.06 \mathrm{E}-02$ & $2.04 \mathrm{E}+20$ & 2.87E-02 \\
\hline $1.60 \mathrm{E}-01$ & 21.53 & 4.47E+19 & $1.20 \mathrm{E}-02$ & $1.21 \mathrm{E}+20$ & $3.26 \mathrm{E}-02$ \\
\hline $1.90 \mathrm{E}-01$ & 15.85 & $6.08 \mathrm{E}+19$ & $1.21 \mathrm{E}-02$ & $1.65 \mathrm{E}+20$ & 3.27E-02 \\
\hline $2.20 \mathrm{E}-01$ & 22.01 & $4.86 \mathrm{E}+19$ & $1.34 \mathrm{E}-02$ & $1.32 \mathrm{E}+20$ & $3.63 E-02$ \\
\hline
\end{tabular}




\begin{tabular}{|c|c|c|c|c|c|}
\hline \multirow{2}{*}{$\begin{array}{c}\text { Upper } \\
\text { Energy(MeV) }\end{array}$} & \multirow{2}{*}{$\begin{array}{l}\text { DPA xsec } \\
\text { keV-barn }\end{array}$} & \multicolumn{2}{|c|}{$6 A$} & \multicolumn{2}{|c|}{ 6-B } \\
\hline & & Total Fluence & DPA & Total Fluence & DPA \\
\hline $2.55 \mathrm{E}-01$ & 18.27 & $5.71 E+19$ & $1.30 \mathrm{E}-02$ & $1.55 E+20$ & 3.53E-02 \\
\hline $2.90 \mathrm{E}-01$ & 17.37 & $6.27 E+19$ & $1.36 \mathrm{E}-02$ & $1.70 \mathrm{E}+20$ & 3.69E-02 \\
\hline $3.20 \mathrm{E}-01$ & 13.51 & $4.32 \mathrm{E}+19$ & $7.30 \mathrm{E}-03$ & 1.17E+20 & $1.98 \mathrm{E}-02$ \\
\hline $3.60 \mathrm{E}-01$ & 25.14 & $6.18 \mathrm{E}+19$ & $1.94 \mathrm{E}-02$ & 1.67E+20 & $5.26 \mathrm{E}-02$ \\
\hline 4.00E-01 & 46.52 & $5.38 \mathrm{E}+19$ & $3.13 \mathrm{E}-02$ & $1.46 \mathrm{E}+20$ & $8.48 \mathrm{E}-02$ \\
\hline $4.50 \mathrm{E}-01$ & 43.04 & $5.07 E+19$ & $2.73 \mathrm{E}-02$ & $1.37 \mathrm{E}+20$ & 7.39E-02 \\
\hline $5.00 \mathrm{E}-01$ & 36.4 & $5.82 \mathrm{E}+19$ & $2.65 \mathrm{E}-02$ & $1.58 \mathrm{E}+20$ & $7.18 \mathrm{E}-02$ \\
\hline $5.50 \mathrm{E}-01$ & 33.22 & $5.75 E+19$ & $2.39 \mathrm{E}-02$ & $1.56 \mathrm{E}+20$ & 6.47E-02 \\
\hline $6.00 \mathrm{E}-01$ & 34.18 & $5.30 \mathrm{E}+19$ & $2.27 \mathrm{E}-02$ & $1.44 \mathrm{E}+20$ & $6.14 \mathrm{E}-02$ \\
\hline $6.60 \mathrm{E}-01$ & 19.64 & $6.80 E+19$ & 1.67E-02 & $1.84 \mathrm{E}+20$ & 4.53E-02 \\
\hline 7.20E-01 & 48.89 & $6.87 E+19$ & 4.20E-02 & $1.86 \mathrm{E}+20$ & 1.14E-01 \\
\hline $7.80 \mathrm{E}-01$ & 74.22 & $6.51 \mathrm{E}+19$ & $6.04 \mathrm{E}-02$ & $1.76 \mathrm{E}+20$ & $1.64 \mathrm{E}-01$ \\
\hline $8.40 \mathrm{E}-01$ & 44.02 & $5.19 E+19$ & $2.86 \mathrm{E}-02$ & $1.41 \mathrm{E}+20$ & 7.74E-02 \\
\hline $9.20 \mathrm{E}-01$ & 40.97 & $6.19 \mathrm{E}+19$ & $3.17 \mathrm{E}-02$ & $1.68 \mathrm{E}+20$ & $8.60 \mathrm{E}-02$ \\
\hline $1.00 \mathrm{E}+00$ & 50.95 & $5.79 E+19$ & $3.69 \mathrm{E}-02$ & $1.57 \mathrm{E}+20$ & $1.00 \mathrm{E}-01$ \\
\hline $1.20 \mathrm{E}+00$ & 50.09 & $1.35 \mathrm{E}+20$ & $8.44 \mathrm{E}-02$ & $3.66 \mathrm{E}+20$ & $2.29 \mathrm{E}-01$ \\
\hline $1.40 \mathrm{E}+00$ & 64.54 & $1.20 \mathrm{E}+20$ & $9.71 \mathrm{E}-02$ & $3.26 \mathrm{E}+20$ & 2.63E-01 \\
\hline $1.60 \mathrm{E}+00$ & 73.58 & $1.01 \mathrm{E}+20$ & $9.29 \mathrm{E}-02$ & $2.74 \mathrm{E}+20$ & $2.52 \mathrm{E}-01$ \\
\hline $1.80 \mathrm{E}+00$ & 76.46 & $8.92 \mathrm{E}+19$ & $8.53 \mathrm{E}-02$ & $2.42 \mathrm{E}+20$ & $2.31 \mathrm{E}-01$ \\
\hline $2.00 \mathrm{E}+00$ & 95.15 & $7.85 E+19$ & $9.34 \mathrm{E}-02$ & $2.13 E+20$ & 2.53E-01 \\
\hline $2.30 \mathrm{E}+00$ & 93.75 & $9.70 \mathrm{E}+19$ & $1.14 \mathrm{E}-01$ & $2.63 \mathrm{E}+20$ & $3.08 \mathrm{E}-01$ \\
\hline $2.60 \mathrm{E}+00$ & 112.05 & $8.90 \mathrm{E}+19$ & $1.25 \mathrm{E}-01$ & $2.41 \mathrm{E}+20$ & 3.38E-01 \\
\hline $2.90 \mathrm{E}+00$ & 123.55 & $6.93 \mathrm{E}+19$ & $1.07 \mathrm{E}-01$ & $1.88 \mathrm{E}+20$ & 2.90E-01 \\
\hline $3.30 \mathrm{E}+00$ & 133.45 & $6.68 \mathrm{E}+19$ & 1.11E-01 & $1.81 \mathrm{E}+20$ & 3.02E-01 \\
\hline $3.70 \mathrm{E}+00$ & 135.25 & $4.61 \mathrm{E}+19$ & $7.80 \mathrm{E}-02$ & $1.25 \mathrm{E}+20$ & $2.11 \mathrm{E}-01$ \\
\hline $4.10 \mathrm{E}+00$ & 149.55 & $3.50 \mathrm{E}+19$ & $6.55 \mathrm{E}-02$ & $9.50 \mathrm{E}+19$ & $1.78 \mathrm{E}-01$ \\
\hline $4.50 \mathrm{E}+00$ & 158.25 & $2.78 \mathrm{E}+19$ & $5.50 \mathrm{E}-02$ & $7.53 E+19$ & $1.49 \mathrm{E}-01$ \\
\hline $5.00 \mathrm{E}+00$ & 168.55 & $2.65 E+19$ & $5.57 \mathrm{E}-02$ & 7.17E+19 & $1.51 \mathrm{E}-01$ \\
\hline $5.50 \mathrm{E}+00$ & 176.46 & $1.76 \mathrm{E}+19$ & $3.88 \mathrm{E}-02$ & 4.77E+19 & $1.05 \mathrm{E}-01$ \\
\hline
\end{tabular}




\begin{tabular}{|c|c|c|c|c|c|}
\hline & DPA xsec & \multicolumn{2}{|c|}{$6 \mathrm{c}$} & \multicolumn{2}{c|}{$6-\mathrm{B}$} \\
\hline $\begin{array}{c}\text { Upper } \\
\text { Energy(MeV) }\end{array}$ & keV-barn & Total Fluence & DPA & Total Fluence & DPA \\
\hline $6.00 \mathrm{E}+00$ & 183.06 & $1.32 \mathrm{E}+19$ & $3.02 \mathrm{E}-02$ & $3.58 \mathrm{E}+19$ & $8.18 \mathrm{E}-02$ \\
\hline $6.70 \mathrm{E}+00$ & 189.26 & $1.13 \mathrm{E}+19$ & $2.67 \mathrm{E}-02$ & $3.06 \mathrm{E}+19$ & $7.23 \mathrm{E}-02$ \\
\hline $7.40 \mathrm{E}+00$ & 196.67 & $6.47 \mathrm{E}+18$ & $1.59 \mathrm{E}-02$ & $1.75 \mathrm{E}+19$ & $4.31 \mathrm{E}-02$ \\
\hline $8.20 \mathrm{E}+00$ & 203.37 & $4.31 \mathrm{E}+18$ & $1.10 \mathrm{E}-02$ & $1.17 \mathrm{E}+19$ & $2.97 \mathrm{E}-02$ \\
\hline $9.00 \mathrm{E}+00$ & 214.58 & $2.43 \mathrm{E}+18$ & $6.51 \mathrm{E}-03$ & $6.58 \mathrm{E}+18$ & $1.77 \mathrm{E}-02$ \\
\hline $1.00 \mathrm{E}+01$ & 225.69 & $1.45 \mathrm{E}+18$ & $4.08 \mathrm{E}-03$ & $3.92 \mathrm{E}+18$ & $1.11 \mathrm{E}-02$ \\
\hline $1.10 \mathrm{E}+01$ & 237.6 & $6.57 \mathrm{E}+17$ & $1.95 \mathrm{E}-03$ & $1.78 \mathrm{E}+18$ & $5.29 \mathrm{E}-03$ \\
\hline $1.20 \mathrm{E}+01$ & 247.41 & $2.39 \mathrm{E}+17$ & $7.40 \mathrm{E}-04$ & $6.49 \mathrm{E}+17$ & $2.01 \mathrm{E}-03$ \\
\hline $1.30 \mathrm{E}+01$ & 258.52 & $1.49 \mathrm{E}+17$ & $4.81 \mathrm{E}-04$ & $4.03 \mathrm{E}+17$ & $1.30 \mathrm{E}-03$ \\
\hline $1.40 \mathrm{E}+01$ & 271.35 & $7.66 \mathrm{E}+16$ & $2.60 \mathrm{E}-04$ & $2.08 \mathrm{E}+17$ & $7.04 \mathrm{E}-04$ \\
\hline $1.50 \mathrm{E}+01$ & 290.27 & $2.75 \mathrm{E}+16$ & $9.99 \mathrm{E}-05$ & $7.46 \mathrm{E}+16$ & $2.71 \mathrm{E}-04$ \\
\hline $1.60 \mathrm{E}+01$ & 293.2 & $2.01 \mathrm{E}+16$ & $7.38 \mathrm{E}-05$ & $5.45 \mathrm{E}+16$ & $2.00 \mathrm{E}-04$ \\
\hline $1.70 \mathrm{E}+01$ & 292.73 & $0.00 \mathrm{E}+00$ & $0.00 \mathrm{E}+00$ & $0.00 \mathrm{E}+00$ & $0.00 \mathrm{E}+00$ \\
\hline $1.80 \mathrm{E}+01$ & 297.65 & $0.00 \mathrm{E}+00$ & $0.00 \mathrm{E}+00$ & $0.00 \mathrm{E}+00$ & $0.00 \mathrm{E}+00$ \\
\hline $1.90 \mathrm{E}+01$ & 307.26 & $0.00 \mathrm{E}+00$ & $0.00 \mathrm{E}+00$ & $0.00 \mathrm{E}+00$ & $0.00 \mathrm{E}+00$ \\
\hline $2.00 \mathrm{E}+01$ & 316.36 & $0.00 \mathrm{E}+00$ & $0.00 \mathrm{E}+00$ & $0.00 \mathrm{E}+00$ & $0.00 \mathrm{E}+00$ \\
\hline & & & $1.82 \mathrm{E}+00$ & & $4.94 \mathrm{E}+00$ \\
\hline
\end{tabular}


Table 16. Calculated fluence and DPA for capsule 5 of the UCSB experiment.

\begin{tabular}{|c|c|c|c|c|c|c|c|c|c|}
\hline \multirow{2}{*}{$\begin{array}{c}\text { Upper } \\
\text { Energy(MeV) }\end{array}$} & \multirow{2}{*}{$\begin{array}{l}\text { DPA xsec } \\
\text { keV-barn }\end{array}$} & \multicolumn{2}{|c|}{$5-4$} & \multicolumn{2}{|c|}{$5-3$} & \multicolumn{2}{|c|}{$5-2$} & \multicolumn{2}{|c|}{$5-1$} \\
\hline & & Total Fluence & DPA & Total Fluence & DPA & Total Fluence & DPA & Total Fluence & DPA \\
\hline $1.00 \mathrm{E}-10$ & 0 & $3.04 \mathrm{E}+15$ & & $3.04 \mathrm{E}+15$ & & $5.59 \mathrm{E}+15$ & & $2.94 \mathrm{E}+15$ & \\
\hline $1.00 \mathrm{E}-09$ & 7.73 & 8.07E+17 & $7.80 \mathrm{E}-05$ & $7.77 \mathrm{E}+17$ & $7.51 \mathrm{E}-05$ & $9.15 \mathrm{E}+17$ & $8.84 \mathrm{E}-05$ & $6.79 \mathrm{E}+17$ & $6.56 \mathrm{E}-05$ \\
\hline $1.00 \mathrm{E}-08$ & 2.444 & $1.25 \mathrm{E}+20$ & $3.82 \mathrm{E}-03$ & $1.26 \mathrm{E}+20$ & $3.85 \mathrm{E}-03$ & $1.22 \mathrm{E}+20$ & $3.72 \mathrm{E}-03$ & $1.20 \mathrm{E}+20$ & $3.65 \mathrm{E}-03$ \\
\hline $2.30 \mathrm{E}-08$ & 1.278 & $4.50 \mathrm{E}+20$ & 7.19E-03 & $4.42 \mathrm{E}+20$ & $7.06 \mathrm{E}-03$ & $4.37 E+20$ & 6.99E-03 & $4.37 E+20$ & $6.98 \mathrm{E}-03$ \\
\hline $5.00 \mathrm{E}-08$ & 0.857 & $1.12 \mathrm{E}+21$ & $1.20 \mathrm{E}-02$ & $1.10 \mathrm{E}+21$ & $1.17 \mathrm{E}-02$ & $1.09 \mathrm{E}+21$ & $1.17 \mathrm{E}-02$ & $1.09 \mathrm{E}+21$ & 1.17E-02 \\
\hline $7.60 \mathrm{E}-08$ & 0.644 & $7.99 \mathrm{E}+20$ & $6.43 \mathrm{E}-03$ & $7.86 \mathrm{E}+20$ & $6.33 \mathrm{E}-03$ & $7.78 \mathrm{E}+20$ & $6.27 \mathrm{E}-03$ & $7.78 \mathrm{E}+20$ & $6.26 \mathrm{E}-03$ \\
\hline $1.15 \mathrm{E}-07$ & 0.523 & $6.89 \mathrm{E}+20$ & $4.50 \mathrm{E}-03$ & $6.83 \mathrm{E}+20$ & 4.47E-03 & $6.68 \mathrm{E}+20$ & 4.37E-03 & $6.70 \mathrm{E}+20$ & 4.38E-03 \\
\hline $1.70 \mathrm{E}-07$ & 0.428 & $4.36 \mathrm{E}+20$ & $2.33 \mathrm{E}-03$ & $4.29 \mathrm{E}+20$ & $2.30 \mathrm{E}-03$ & $4.19 E+20$ & $2.24 \mathrm{E}-03$ & $4.24 \mathrm{E}+20$ & $2.27 \mathrm{E}-03$ \\
\hline $2.55 \mathrm{E}-07$ & 0.351 & $3.02 E+20$ & $1.33 \mathrm{E}-03$ & $3.04 \mathrm{E}+20$ & $1.33 \mathrm{E}-03$ & $2.98 \mathrm{E}+20$ & $1.31 \mathrm{E}-03$ & $2.94 \mathrm{E}+20$ & $1.29 \mathrm{E}-03$ \\
\hline $3.80 \mathrm{E}-07$ & 0.287 & $2.56 \mathrm{E}+20$ & $9.18 \mathrm{E}-04$ & $2.53 \mathrm{E}+20$ & $9.08 \mathrm{E}-04$ & $2.52 \mathrm{E}+20$ & $9.03 E-04$ & $2.52 \mathrm{E}+20$ & $9.04 \mathrm{E}-04$ \\
\hline $5.50 \mathrm{E}-07$ & 0.237 & $2.29 E+20$ & $6.78 \mathrm{E}-04$ & $2.29 \mathrm{E}+20$ & $6.80 \mathrm{E}-04$ & $2.32 \mathrm{E}+20$ & $6.87 \mathrm{E}-04$ & $2.29 \mathrm{E}+20$ & $6.80 \mathrm{E}-04$ \\
\hline $8.40 \mathrm{E}-07$ & 0.194 & $2.59 \mathrm{E}+20$ & $6.27 \mathrm{E}-04$ & $2.57 \mathrm{E}+20$ & $6.24 \mathrm{E}-04$ & $2.57 \mathrm{E}+20$ & $6.23 \mathrm{E}-04$ & $2.54 \mathrm{E}+20$ & 6.17E-04 \\
\hline $1.28 \mathrm{E}-06$ & 0.157 & $2.46 \mathrm{E}+20$ & 4.83E-04 & $2.47 E+20$ & $4.85 \mathrm{E}-04$ & $2.44 \mathrm{E}+20$ & $4.80 \mathrm{E}-04$ & $2.49 \mathrm{E}+20$ & $4.88 \mathrm{E}-04$ \\
\hline $1.90 \mathrm{E}-06$ & 0.128 & $2.34 \mathrm{E}+20$ & $3.74 \mathrm{E}-04$ & $2.31 \mathrm{E}+20$ & $3.70 \mathrm{E}-04$ & $2.29 \mathrm{E}+20$ & 3.67E-04 & $2.27 \mathrm{E}+20$ & 3.63E-04 \\
\hline $2.80 \mathrm{E}-06$ & 0.105 & $2.26 \mathrm{E}+20$ & $2.96 \mathrm{E}-04$ & $2.28 \mathrm{E}+20$ & $2.99 \mathrm{E}-04$ & $2.25 E+20$ & $2.95 \mathrm{E}-04$ & $2.18 \mathrm{E}+20$ & $2.86 \mathrm{E}-04$ \\
\hline 4.25E-06 & 0.086 & $2.41 \mathrm{E}+20$ & $2.59 \mathrm{E}-04$ & $2.37 \mathrm{E}+20$ & $2.55 \mathrm{E}-04$ & $2.35 \mathrm{E}+20$ & $2.53 \mathrm{E}-04$ & $2.34 \mathrm{E}+20$ & $2.52 \mathrm{E}-04$ \\
\hline $6.30 \mathrm{E}-06$ & 0.07 & $2.21 \mathrm{E}+20$ & $1.94 \mathrm{E}-04$ & $2.24 \mathrm{E}+20$ & $1.96 \mathrm{E}-04$ & $2.17 \mathrm{E}+20$ & $1.90 \mathrm{E}-04$ & $2.15 \mathrm{E}+20$ & $1.88 \mathrm{E}-04$ \\
\hline $9.20 \mathrm{E}-06$ & 0.058 & $2.13 E+20$ & $1.55 \mathrm{E}-04$ & $2.11 \mathrm{E}+20$ & $1.53 \mathrm{E}-04$ & $2.09 \mathrm{E}+20$ & $1.52 \mathrm{E}-04$ & $2.14 \mathrm{E}+20$ & $1.55 \mathrm{E}-04$ \\
\hline $1.35 \mathrm{E}-05$ & 0.048 & $2.25 \mathrm{E}+20$ & $1.35 \mathrm{E}-04$ & $2.21 \mathrm{E}+20$ & $1.33 \mathrm{E}-04$ & $2.21 \mathrm{E}+20$ & $1.33 \mathrm{E}-04$ & $2.17 \mathrm{E}+20$ & $1.30 \mathrm{E}-04$ \\
\hline $2.10 \mathrm{E}-05$ & 0.038 & $2.48 \mathrm{E}+20$ & $1.18 \mathrm{E}-04$ & $2.53 \mathrm{E}+20$ & $1.20 \mathrm{E}-04$ & $2.49 \mathrm{E}+20$ & $1.18 \mathrm{E}-04$ & $2.46 \mathrm{E}+20$ & 1.17E-04 \\
\hline $3.00 \mathrm{E}-05$ & 0.031 & $2.07 \mathrm{E}+20$ & 8.03E-05 & $2.08 \mathrm{E}+20$ & 8.05E-05 & $2.04 \mathrm{E}+20$ & 7.89E-05 & $2.03 \mathrm{E}+20$ & $7.88 \mathrm{E}-05$ \\
\hline $4.50 \mathrm{E}-05$ & 0.026 & $2.40 \mathrm{E}+20$ & 7.79E-05 & $2.44 \mathrm{E}+20$ & $7.92 \mathrm{E}-05$ & $2.37 \mathrm{E}+20$ & 7.71E-05 & $2.33 \mathrm{E}+20$ & 7.59E-05 \\
\hline $6.90 \mathrm{E}-05$ & 0.021 & $2.56 \mathrm{E}+20$ & $6.71 \mathrm{E}-05$ & $2.58 \mathrm{E}+20$ & $6.76 \mathrm{E}-05$ & $2.55 \mathrm{E}+20$ & $6.70 \mathrm{E}-05$ & $2.56 \mathrm{E}+20$ & $6.73 \mathrm{E}-05$ \\
\hline $1.00 \mathrm{E}-04$ & 0.017 & $2.27 \mathrm{E}+20$ & $4.82 \mathrm{E}-05$ & $2.30 \mathrm{E}+20$ & $4.89 \mathrm{E}-05$ & $2.28 \mathrm{E}+20$ & $4.84 \mathrm{E}-05$ & $2.22 \mathrm{E}+20$ & 4.71E-05 \\
\hline $1.35 \mathrm{E}-04$ & 0.014 & $1.80 \mathrm{E}+20$ & $3.14 \mathrm{E}-05$ & $1.83 \mathrm{E}+20$ & $3.21 \mathrm{E}-05$ & $1.81 \mathrm{E}+20$ & 3.17E-05 & $1.79 \mathrm{E}+20$ & 3.13E-05 \\
\hline $1.70 \mathrm{E}-04$ & 0.014 & $1.42 \mathrm{E}+20$ & $2.48 \mathrm{E}-05$ & $1.42 \mathrm{E}+20$ & $2.49 \mathrm{E}-05$ & $1.42 \mathrm{E}+20$ & 2.49E-05 & $1.42 \mathrm{E}+20$ & $2.49 \mathrm{E}-05$ \\
\hline $2.20 \mathrm{E}-04$ & 0.011 & $1.61 \mathrm{E}+20$ & $2.21 \mathrm{E}-05$ & $1.59 \mathrm{E}+20$ & $2.18 \mathrm{E}-05$ & $1.59 \mathrm{E}+20$ & 2.19E-05 & $1.61 \mathrm{E}+20$ & $2.21 \mathrm{E}-05$ \\
\hline
\end{tabular}




\begin{tabular}{|c|c|c|c|c|c|c|c|c|c|}
\hline \multirow[b]{2}{*}{$\begin{array}{c}\text { Upper } \\
\text { Energy(MeV) } \\
\end{array}$} & \multirow{2}{*}{$\begin{array}{l}\text { DPA xsec } \\
\text { keV-barn }\end{array}$} & \multicolumn{2}{|c|}{$5-4$} & \multicolumn{2}{|c|}{$5-3$} & \multicolumn{2}{|c|}{$5-2$} & \multicolumn{2}{|c|}{$5-1$} \\
\hline & & Total Fluence & DPA & Total Fluence & DPA & Total Fluence & DPA & Total Fluence & DPA \\
\hline $2.80 \mathrm{E}-04$ & 0.011 & $1.53 \mathrm{E}+20$ & $2.11 \mathrm{E}-05$ & $1.49 \mathrm{E}+20$ & 2.05E-05 & $1.53 E+20$ & $2.10 \mathrm{E}-05$ & $1.53 E+20$ & $2.10 \mathrm{E}-05$ \\
\hline $3.60 \mathrm{E}-04$ & 0.01 & $1.57 \mathrm{E}+20$ & $1.96 \mathrm{E}-05$ & $1.54 \mathrm{E}+20$ & $1.93 \mathrm{E}-05$ & $1.52 \mathrm{E}+20$ & $1.90 \mathrm{E}-05$ & $1.50 \mathrm{E}+20$ & $1.88 \mathrm{E}-05$ \\
\hline $4.50 \mathrm{E}-04$ & 0.007 & $1.37 \mathrm{E}+20$ & 1.20E-05 & $1.38 \mathrm{E}+20$ & $1.21 \mathrm{E}-05$ & $1.34 \mathrm{E}+20$ & 1.17E-05 & $1.33 \mathrm{E}+20$ & 1.16E-05 \\
\hline $5.75 \mathrm{E}-04$ & 0.005 & $1.53 \mathrm{E}+20$ & $9.55 \mathrm{E}-06$ & $1.53 \mathrm{E}+20$ & $9.58 \mathrm{E}-06$ & $1.58 \mathrm{E}+20$ & $9.85 \mathrm{E}-06$ & $1.54 \mathrm{E}+20$ & 9.63E-06 \\
\hline 7.60E-04 & 0.129 & $1.77 \mathrm{E}+20$ & $2.85 \mathrm{E}-04$ & $1.80 \mathrm{E}+20$ & $2.91 \mathrm{E}-04$ & 1.77E+20 & $2.86 \mathrm{E}-04$ & $1.76 \mathrm{E}+20$ & $2.84 \mathrm{E}-04$ \\
\hline $9.60 \mathrm{E}-04$ & 0.308 & $1.48 \mathrm{E}+20$ & 5.69E-04 & $1.46 \mathrm{E}+20$ & 5.61E-04 & $1.47 \mathrm{E}+20$ & 5.66E-04 & $1.43 \mathrm{E}+20$ & $5.51 \mathrm{E}-04$ \\
\hline $1.28 \mathrm{E}-03$ & 0.672 & $1.80 \mathrm{E}+20$ & $1.51 \mathrm{E}-03$ & $1.77 \mathrm{E}+20$ & $1.49 \mathrm{E}-03$ & $1.79 \mathrm{E}+20$ & $1.50 \mathrm{E}-03$ & $1.73 \mathrm{E}+20$ & $1.45 \mathrm{E}-03$ \\
\hline $1.60 \mathrm{E}-03$ & 0.477 & $1.45 \mathrm{E}+20$ & 8.67E-04 & $1.48 \mathrm{E}+20$ & $8.80 \mathrm{E}-04$ & $1.39 \mathrm{E}+20$ & $8.30 \mathrm{E}-04$ & $1.40 \mathrm{E}+20$ & $8.35 \mathrm{E}-04$ \\
\hline $2.00 \mathrm{E}-03$ & 0.511 & $1.46 \mathrm{E}+20$ & $9.32 \mathrm{E}-04$ & $1.41 \mathrm{E}+20$ & $9.02 \mathrm{E}-04$ & $1.45 \mathrm{E}+20$ & 9.24E-04 & $1.40 \mathrm{E}+20$ & $8.95 \mathrm{E}-04$ \\
\hline $2.70 \mathrm{E}-03$ & 0.568 & $1.94 \mathrm{E}+20$ & $1.38 \mathrm{E}-03$ & $1.86 \mathrm{E}+20$ & $1.32 \mathrm{E}-03$ & $1.92 \mathrm{E}+20$ & $1.37 \mathrm{E}-03$ & $1.89 \mathrm{E}+20$ & $1.34 \mathrm{E}-03$ \\
\hline $3.40 \mathrm{E}-03$ & 0.632 & $1.52 \mathrm{E}+20$ & $1.20 \mathrm{E}-03$ & $1.52 \mathrm{E}+20$ & $1.20 \mathrm{E}-03$ & $1.49 \mathrm{E}+20$ & $1.18 \mathrm{E}-03$ & $1.48 \mathrm{E}+20$ & 1.17E-03 \\
\hline $4.50 \mathrm{E}-03$ & 0.822 & $1.84 \mathrm{E}+20$ & $1.89 \mathrm{E}-03$ & $1.87 \mathrm{E}+20$ & $1.92 \mathrm{E}-03$ & $1.86 \mathrm{E}+20$ & $1.92 \mathrm{E}-03$ & $1.80 \mathrm{E}+20$ & $1.85 \mathrm{E}-03$ \\
\hline $5.50 \mathrm{E}-03$ & 0.826 & $1.43 \mathrm{E}+20$ & 1.47E-03 & $1.41 \mathrm{E}+20$ & $1.45 \mathrm{E}-03$ & $1.37 \mathrm{E}+20$ & $1.41 \mathrm{E}-03$ & $1.39 \mathrm{E}+20$ & $1.44 \mathrm{E}-03$ \\
\hline $7.20 \mathrm{E}-03$ & 1.671 & $1.85 \mathrm{E}+20$ & $3.86 \mathrm{E}-03$ & $1.88 \mathrm{E}+20$ & 3.93E-03 & $1.83 \mathrm{E}+20$ & $3.81 \mathrm{E}-03$ & $1.78 \mathrm{E}+20$ & $3.72 \mathrm{E}-03$ \\
\hline $9.20 \mathrm{E}-03$ & 3.709 & $1.49 \mathrm{E}+20$ & $6.91 \mathrm{E}-03$ & $1.48 \mathrm{E}+20$ & $6.84 \mathrm{E}-03$ & $1.46 \mathrm{E}+20$ & $6.77 \mathrm{E}-03$ & $1.48 \mathrm{E}+20$ & $6.88 \mathrm{E}-03$ \\
\hline $1.20 \mathrm{E}-02$ & 1.491 & $1.82 \mathrm{E}+20$ & $3.38 \mathrm{E}-03$ & $1.86 \mathrm{E}+20$ & 3.47E-03 & $1.80 \mathrm{E}+20$ & $3.35 \mathrm{E}-03$ & $1.76 \mathrm{E}+20$ & $3.28 \mathrm{E}-03$ \\
\hline $1.50 \mathrm{E}-02$ & 1.156 & $1.59 \mathrm{E}+20$ & 2.29E-03 & $1.60 \mathrm{E}+20$ & $2.31 \mathrm{E}-03$ & $1.56 \mathrm{E}+20$ & $2.26 \mathrm{E}-03$ & $1.53 \mathrm{E}+20$ & $2.21 \mathrm{E}-03$ \\
\hline $1.90 \mathrm{E}-02$ & 0.973 & $1.60 \mathrm{E}+20$ & $1.94 \mathrm{E}-03$ & $1.63 \mathrm{E}+20$ & $1.98 \mathrm{E}-03$ & $1.57 \mathrm{E}+20$ & $1.91 \mathrm{E}-03$ & $1.58 \mathrm{E}+20$ & $1.93 \mathrm{E}-03$ \\
\hline $2.55 \mathrm{E}-02$ & 0.555 & $2.42 \mathrm{E}+20$ & $1.68 \mathrm{E}-03$ & $2.45 \mathrm{E}+20$ & $1.70 \mathrm{E}-03$ & $2.41 \mathrm{E}+20$ & 1.67E-03 & $2.31 \mathrm{E}+20$ & $1.60 \mathrm{E}-03$ \\
\hline $3.20 \mathrm{E}-02$ & 26.762 & $2.15 \mathrm{E}+20$ & 7.19E-02 & $2.14 \mathrm{E}+20$ & 7.16E-02 & $2.16 \mathrm{E}+20$ & 7.23E-02 & $2.12 \mathrm{E}+20$ & $7.08 \mathrm{E}-02$ \\
\hline $4.00 \mathrm{E}-02$ & 8.161 & $1.02 \mathrm{E}+20$ & $1.04 \mathrm{E}-02$ & $1.04 \mathrm{E}+20$ & 1.06E-02 & $1.02 \mathrm{E}+20$ & $1.05 \mathrm{E}-02$ & $1.02 \mathrm{E}+20$ & $1.04 \mathrm{E}-02$ \\
\hline $5.25 \mathrm{E}-02$ & 6.515 & $2.10 \mathrm{E}+20$ & $1.71 \mathrm{E}-02$ & $2.08 \mathrm{E}+20$ & $1.69 \mathrm{E}-02$ & $2.07 \mathrm{E}+20$ & $1.69 \mathrm{E}-02$ & $2.04 \mathrm{E}+20$ & $1.66 \mathrm{E}-02$ \\
\hline $6.60 \mathrm{E}-02$ & 6.576 & $2.11 \mathrm{E}+20$ & $1.73 \mathrm{E}-02$ & $2.09 \mathrm{E}+20$ & $1.72 \mathrm{E}-02$ & $2.06 \mathrm{E}+20$ & $1.70 \mathrm{E}-02$ & $2.01 \mathrm{E}+20$ & $1.65 \mathrm{E}-02$ \\
\hline $8.80 \mathrm{E}-02$ & 13.137 & $3.05 E+20$ & $5.00 \mathrm{E}-02$ & $3.06 \mathrm{E}+20$ & $5.02 \mathrm{E}-02$ & $3.06 \mathrm{E}+20$ & $5.02 \mathrm{E}-02$ & $3.03 E+20$ & 4.97E-02 \\
\hline $1.10 \mathrm{E}-01$ & 11.84 & $1.85 \mathrm{E}+20$ & $2.74 \mathrm{E}-02$ & $1.86 \mathrm{E}+20$ & $2.76 \mathrm{E}-02$ & $1.83 \mathrm{E}+20$ & $2.71 \mathrm{E}-02$ & $1.80 \mathrm{E}+20$ & 2.67E-02 \\
\hline $1.35 \mathrm{E}-01$ & 11.27 & $2.85 \mathrm{E}+20$ & 4.02E-02 & $2.77 \mathrm{E}+20$ & $3.91 \mathrm{E}-02$ & $2.79 \mathrm{E}+20$ & $3.93 \mathrm{E}-02$ & $2.68 \mathrm{E}+20$ & $3.78 \mathrm{E}-02$ \\
\hline $1.60 \mathrm{E}-01$ & 21.53 & $1.64 \mathrm{E}+20$ & 4.41E-02 & $1.62 \mathrm{E}+20$ & 4.35E-02 & $1.63 \mathrm{E}+20$ & $4.38 \mathrm{E}-02$ & $1.57 \mathrm{E}+20$ & $4.23 \mathrm{E}-02$ \\
\hline $1.90 \mathrm{E}-01$ & 15.85 & $2.26 \mathrm{E}+20$ & 4.47E-02 & $2.23 \mathrm{E}+20$ & 4.42E-02 & $2.23 \mathrm{E}+20$ & 4.42E-02 & $2.23 \mathrm{E}+20$ & $4.41 \mathrm{E}-02$ \\
\hline $2.20 \mathrm{E}-01$ & 22.01 & $1.83 \mathrm{E}+20$ & $5.05 \mathrm{E}-02$ & $1.86 \mathrm{E}+20$ & $5.12 \mathrm{E}-02$ & $1.85 \mathrm{E}+20$ & $5.08 \mathrm{E}-02$ & $1.79 \mathrm{E}+20$ & 4.93E-02 \\
\hline
\end{tabular}




\begin{tabular}{|c|c|c|c|c|c|c|c|c|c|}
\hline \multirow[b]{2}{*}{$\begin{array}{c}\text { Upper } \\
\text { Energy(MeV) }\end{array}$} & \multirow{2}{*}{$\begin{array}{l}\text { DPA xsec } \\
\text { keV-barn }\end{array}$} & \multicolumn{2}{|c|}{$5-4$} & \multicolumn{2}{|c|}{$5-3$} & \multicolumn{2}{|c|}{$5-2$} & \multicolumn{2}{|c|}{$5-1$} \\
\hline & & Total Fluence & DPA & Total Fluence & DPA & Total Fluence & DPA & Total Fluence & DPA \\
\hline $2.55 \mathrm{E}-01$ & 18.27 & $2.17 \mathrm{E}+20$ & 4.96E-02 & $2.19 \mathrm{E}+20$ & $5.01 \mathrm{E}-02$ & $2.14 \mathrm{E}+20$ & $4.88 \mathrm{E}-02$ & $2.12 \mathrm{E}+20$ & $4.84 \mathrm{E}-02$ \\
\hline $2.90 \mathrm{E}-01$ & 17.37 & $2.32 \mathrm{E}+20$ & $5.04 \mathrm{E}-02$ & $2.29 \mathrm{E}+20$ & 4.98E-02 & $2.32 \mathrm{E}+20$ & 5.03E-02 & $2.31 \mathrm{E}+20$ & $5.02 \mathrm{E}-02$ \\
\hline $3.20 \mathrm{E}-01$ & 13.51 & $1.65 \mathrm{E}+20$ & $2.78 \mathrm{E}-02$ & $1.65 \mathrm{E}+20$ & $2.78 \mathrm{E}-02$ & $1.65 \mathrm{E}+20$ & $2.78 \mathrm{E}-02$ & $1.62 \mathrm{E}+20$ & $2.74 \mathrm{E}-02$ \\
\hline $3.60 \mathrm{E}-01$ & 25.14 & $2.31 \mathrm{E}+20$ & 7.26E-02 & $2.33 \mathrm{E}+20$ & 7.31E-02 & $2.27 \mathrm{E}+20$ & 7.14E-02 & $2.27 \mathrm{E}+20$ & 7.15E-02 \\
\hline 4.00E-01 & 46.52 & $2.01 \mathrm{E}+20$ & 1.17E-01 & $1.98 \mathrm{E}+20$ & 1.15E-01 & $2.01 \mathrm{E}+20$ & 1.17E-01 & $1.98 \mathrm{E}+20$ & $1.15 \mathrm{E}-01$ \\
\hline $4.50 \mathrm{E}-01$ & 43.04 & $1.87 \mathrm{E}+20$ & $1.00 \mathrm{E}-01$ & $1.89 \mathrm{E}+20$ & $1.02 \mathrm{E}-01$ & $1.85 \mathrm{E}+20$ & $9.96 \mathrm{E}-02$ & $1.85 \mathrm{E}+20$ & $9.96 \mathrm{E}-02$ \\
\hline 5.00E-01 & 36.4 & $2.14 \mathrm{E}+20$ & $9.72 \mathrm{E}-02$ & $2.15 \mathrm{E}+20$ & $9.79 \mathrm{E}-02$ & $2.09 \mathrm{E}+20$ & $9.51 \mathrm{E}-02$ & $2.11 \mathrm{E}+20$ & $9.58 \mathrm{E}-02$ \\
\hline $5.50 \mathrm{E}-01$ & 33.22 & $2.10 \mathrm{E}+20$ & $8.72 \mathrm{E}-02$ & $2.14 \mathrm{E}+20$ & $8.89 \mathrm{E}-02$ & $2.10 \mathrm{E}+20$ & 8.74E-02 & $2.09 \mathrm{E}+20$ & 8.67E-02 \\
\hline $6.00 \mathrm{E}-01$ & 34.18 & $2.03 E+20$ & 8.67E-02 & $1.98 \mathrm{E}+20$ & $8.45 \mathrm{E}-02$ & $1.98 \mathrm{E}+20$ & 8.44E-02 & $1.98 \mathrm{E}+20$ & $8.48 \mathrm{E}-02$ \\
\hline $6.60 \mathrm{E}-01$ & 19.64 & $2.48 \mathrm{E}+20$ & $6.08 \mathrm{E}-02$ & $2.51 \mathrm{E}+20$ & 6.17E-02 & $2.48 \mathrm{E}+20$ & $6.09 \mathrm{E}-02$ & $2.43 \mathrm{E}+20$ & 5.96E-02 \\
\hline $7.20 \mathrm{E}-01$ & 48.89 & $2.52 \mathrm{E}+20$ & $1.54 \mathrm{E}-01$ & $2.59 \mathrm{E}+20$ & $1.58 \mathrm{E}-01$ & $2.52 \mathrm{E}+20$ & $1.54 \mathrm{E}-01$ & $2.48 \mathrm{E}+20$ & $1.51 \mathrm{E}-01$ \\
\hline $7.80 \mathrm{E}-01$ & 74.22 & $2.43 E+20$ & $2.25 \mathrm{E}-01$ & $2.41 \mathrm{E}+20$ & $2.23 \mathrm{E}-01$ & $2.39 \mathrm{E}+20$ & $2.22 \mathrm{E}-01$ & $2.41 \mathrm{E}+20$ & $2.24 \mathrm{E}-01$ \\
\hline $8.40 \mathrm{E}-01$ & 44.02 & $1.90 \mathrm{E}+20$ & $1.05 \mathrm{E}-01$ & $1.92 \mathrm{E}+20$ & 1.05E-01 & $1.89 \mathrm{E}+20$ & $1.04 \mathrm{E}-01$ & $1.87 \mathrm{E}+20$ & $1.03 \mathrm{E}-01$ \\
\hline $9.20 \mathrm{E}-01$ & 40.97 & $2.32 \mathrm{E}+20$ & 1.19E-01 & $2.32 \mathrm{E}+20$ & 1.19E-01 & $2.28 \mathrm{E}+20$ & 1.17E-01 & $2.25 \mathrm{E}+20$ & $1.15 \mathrm{E}-01$ \\
\hline $1.00 \mathrm{E}+00$ & 50.95 & $2.15 \mathrm{E}+20$ & 1.37E-01 & $2.16 \mathrm{E}+20$ & $1.38 \mathrm{E}-01$ & $2.13 \mathrm{E}+20$ & $1.36 \mathrm{E}-01$ & $2.11 \mathrm{E}+20$ & $1.34 \mathrm{E}-01$ \\
\hline $1.20 \mathrm{E}+00$ & 50.09 & $4.92 \mathrm{E}+20$ & $3.08 \mathrm{E}-01$ & $4.92 \mathrm{E}+20$ & $3.08 \mathrm{E}-01$ & $4.96 \mathrm{E}+20$ & 3.10E-01 & $4.85 \mathrm{E}+20$ & 3.04E-01 \\
\hline $1.40 \mathrm{E}+00$ & 64.54 & $4.50 \mathrm{E}+20$ & 3.63E-01 & $4.42 \mathrm{E}+20$ & 3.57E-01 & $4.43 \mathrm{E}+20$ & $3.58 \mathrm{E}-01$ & $4.39 \mathrm{E}+20$ & $3.54 \mathrm{E}-01$ \\
\hline $1.60 \mathrm{E}+00$ & 73.58 & $3.80 \mathrm{E}+20$ & $3.50 \mathrm{E}-01$ & $3.79 \mathrm{E}+20$ & $3.48 \mathrm{E}-01$ & $3.76 \mathrm{E}+20$ & $3.46 \mathrm{E}-01$ & $3.71 \mathrm{E}+20$ & $3.41 \mathrm{E}-01$ \\
\hline $1.80 \mathrm{E}+00$ & 76.46 & $3.31 \mathrm{E}+20$ & $3.16 \mathrm{E}-01$ & $3.39 \mathrm{E}+20$ & $3.24 \mathrm{E}-01$ & $3.23 \mathrm{E}+20$ & 3.09E-01 & $3.29 \mathrm{E}+20$ & $3.14 \mathrm{E}-01$ \\
\hline $2.00 \mathrm{E}+00$ & 95.15 & $2.84 \mathrm{E}+20$ & $3.38 \mathrm{E}-01$ & $2.90 \mathrm{E}+20$ & 3.44E-01 & $2.91 \mathrm{E}+20$ & 3.47E-01 & $2.88 \mathrm{E}+20$ & 3.43E-01 \\
\hline $2.30 \mathrm{E}+00$ & 93.75 & $3.63 \mathrm{E}+20$ & 4.25E-01 & $3.61 \mathrm{E}+20$ & 4.23E-01 & $3.57 \mathrm{E}+20$ & 4.18E-01 & $3.53 \mathrm{E}+20$ & 4.14E-01 \\
\hline $2.60 \mathrm{E}+00$ & 112.05 & $3.33 E+20$ & 4.66E-01 & $3.33 E+20$ & 4.66E-01 & $3.34 \mathrm{E}+20$ & 4.68E-01 & $3.31 \mathrm{E}+20$ & $4.64 \mathrm{E}-01$ \\
\hline $2.90 \mathrm{E}+00$ & 123.55 & $2.53 \mathrm{E}+20$ & $3.90 \mathrm{E}-01$ & $2.50 \mathrm{E}+20$ & 3.86E-01 & $2.52 \mathrm{E}+20$ & 3.89E-01 & $2.50 \mathrm{E}+20$ & $3.85 \mathrm{E}-01$ \\
\hline $3.30 \mathrm{E}+00$ & 133.45 & $2.49 \mathrm{E}+20$ & 4.15E-01 & $2.52 \mathrm{E}+20$ & 4.21E-01 & $2.47 \mathrm{E}+20$ & $4.12 \mathrm{E}-01$ & $2.47 \mathrm{E}+20$ & 4.11E-01 \\
\hline $3.70 \mathrm{E}+00$ & 135.25 & $1.70 \mathrm{E}+20$ & 2.87E-01 & $1.68 \mathrm{E}+20$ & $2.84 \mathrm{E}-01$ & $1.67 \mathrm{E}+20$ & $2.82 \mathrm{E}-01$ & $1.65 \mathrm{E}+20$ & $2.80 \mathrm{E}-01$ \\
\hline $4.10 \mathrm{E}+00$ & 149.55 & $1.30 \mathrm{E}+20$ & $2.42 \mathrm{E}-01$ & $1.31 \mathrm{E}+20$ & $2.44 \mathrm{E}-01$ & $1.33 \mathrm{E}+20$ & $2.48 \mathrm{E}-01$ & $1.29 \mathrm{E}+20$ & 2.40E-01 \\
\hline $4.50 \mathrm{E}+00$ & 158.25 & $1.02 \mathrm{E}+20$ & $2.01 \mathrm{E}-01$ & $1.03 \mathrm{E}+20$ & $2.04 \mathrm{E}-01$ & $1.01 \mathrm{E}+20$ & $1.99 \mathrm{E}-01$ & $1.02 \mathrm{E}+20$ & $2.01 \mathrm{E}-01$ \\
\hline $5.00 \mathrm{E}+00$ & 168.55 & $9.65 \mathrm{E}+19$ & 2.03E-01 & $9.45 \mathrm{E}+19$ & $1.99 \mathrm{E}-01$ & $9.89 \mathrm{E}+19$ & $2.08 \mathrm{E}-01$ & $9.53 E+19$ & $2.01 \mathrm{E}-01$ \\
\hline $5.50 \mathrm{E}+00$ & 176.46 & $6.62 E+19$ & $1.46 \mathrm{E}-01$ & $6.58 \mathrm{E}+19$ & 1.45E-01 & $6.73 E+19$ & $1.48 \mathrm{E}-01$ & $6.50 \mathrm{E}+19$ & $1.43 \mathrm{E}-01$ \\
\hline
\end{tabular}




\begin{tabular}{|c|c|c|c|c|c|c|c|c|c|}
\hline \multirow{2}{*}{$\begin{array}{c}\text { Upper } \\
\text { Energy(MeV) }\end{array}$} & \multirow{2}{*}{$\begin{array}{l}\text { DPA xsec } \\
\text { keV-barn }\end{array}$} & \multicolumn{2}{|c|}{$5-4$} & \multicolumn{2}{|c|}{$5-3$} & \multicolumn{2}{|c|}{$5-2$} & \multicolumn{2}{|c|}{$5-1$} \\
\hline & & Total Fluence & DPA & Total Fluence & DPA & Total Fluence & DPA & Total Fluence & DPA \\
\hline $6.00 \mathrm{E}+00$ & 183.06 & $4.64 \mathrm{E}+19$ & $1.06 \mathrm{E}-01$ & $4.69 E+19$ & 1.07E-01 & $4.70 E+19$ & $1.08 \mathrm{E}-01$ & $4.48 \mathrm{E}+19$ & $1.03 \mathrm{E}-01$ \\
\hline $6.70 \mathrm{E}+00$ & 189.26 & $4.33 E+19$ & $1.03 \mathrm{E}-01$ & $4.35 E+19$ & 1.03E-01 & $4.24 E+19$ & 1.00E-01 & $4.20 E+19$ & $9.93 \mathrm{E}-02$ \\
\hline $7.40 \mathrm{E}+00$ & 196.67 & $2.40 \mathrm{E}+19$ & $5.91 \mathrm{E}-02$ & $2.54 \mathrm{E}+19$ & $6.24 \mathrm{E}-02$ & $2.35 \mathrm{E}+19$ & $5.78 \mathrm{E}-02$ & $2.40 \mathrm{E}+19$ & $5.89 \mathrm{E}-02$ \\
\hline $8.20 \mathrm{E}+00$ & 203.37 & $1.47 \mathrm{E}+19$ & $3.74 \mathrm{E}-02$ & $1.54 \mathrm{E}+19$ & $3.90 \mathrm{E}-02$ & $1.58 \mathrm{E}+19$ & 4.01E-02 & $1.53 \mathrm{E}+19$ & $3.88 \mathrm{E}-02$ \\
\hline $9.00 \mathrm{E}+00$ & 214.58 & $7.72 \mathrm{E}+18$ & 2.07E-02 & $7.87 \mathrm{E}+18$ & $2.11 \mathrm{E}-02$ & $8.25 \mathrm{E}+18$ & $2.21 \mathrm{E}-02$ & $7.77 \mathrm{E}+18$ & $2.08 \mathrm{E}-02$ \\
\hline $1.00 \mathrm{E}+01$ & 225.69 & $5.14 \mathrm{E}+18$ & $1.45 \mathrm{E}-02$ & $4.79 E+18$ & $1.35 \mathrm{E}-02$ & $5.70 E+18$ & $1.61 \mathrm{E}-02$ & $5.43 E+18$ & $1.53 \mathrm{E}-02$ \\
\hline $1.10 \mathrm{E}+01$ & 237.6 & $2.72 E+18$ & 8.08E-03 & $2.70 \mathrm{E}+18$ & 8.00E-03 & $2.74 \mathrm{E}+18$ & $8.13 \mathrm{E}-03$ & $2.28 \mathrm{E}+18$ & $6.78 \mathrm{E}-03$ \\
\hline $1.20 \mathrm{E}+01$ & 247.41 & $9.87 \mathrm{E}+17$ & 3.05E-03 & $1.07 \mathrm{E}+18$ & 3.30E-03 & $1.11 \mathrm{E}+18$ & 3.43E-03 & $1.54 \mathrm{E}+18$ & 4.76E-03 \\
\hline $1.30 \mathrm{E}+01$ & 258.52 & $5.68 \mathrm{E}+17$ & $1.83 \mathrm{E}-03$ & $5.88 \mathrm{E}+17$ & $1.90 \mathrm{E}-03$ & $6.09 \mathrm{E}+17$ & $1.97 \mathrm{E}-03$ & $4.75 \mathrm{E}+17$ & $1.53 \mathrm{E}-03$ \\
\hline $1.40 \mathrm{E}+01$ & 271.35 & $1.12 \mathrm{E}+17$ & $3.81 \mathrm{E}-04$ & $1.21 \mathrm{E}+17$ & 4.11E-04 & $2.05 \mathrm{E}+17$ & $6.95 \mathrm{E}-04$ & $3.72 \mathrm{E}+17$ & $1.26 \mathrm{E}-03$ \\
\hline $1.50 \mathrm{E}+01$ & 290.27 & $8.59 E+16$ & $3.12 \mathrm{E}-04$ & $8.09 E+16$ & $2.94 \mathrm{E}-04$ & $7.18 \mathrm{E}+16$ & 2.60E-04 & $1.34 \mathrm{E}+17$ & 4.87E-04 \\
\hline $1.60 \mathrm{E}+01$ & 293.2 & $7.08 \mathrm{E}+16$ & $2.59 \mathrm{E}-04$ & $7.88 \mathrm{E}+16$ & $2.89 \mathrm{E}-04$ & $3.32 \mathrm{E}+16$ & $1.22 \mathrm{E}-04$ & $1.01 \mathrm{E}+17$ & $3.69 \mathrm{E}-04$ \\
\hline $1.70 \mathrm{E}+01$ & 292.73 & $4.74 \mathrm{E}+16$ & $1.73 \mathrm{E}-04$ & $8.54 \mathrm{E}+15$ & $3.12 \mathrm{E}-05$ & $2.64 \mathrm{E}+16$ & 9.67E-05 & $6.96 \mathrm{E}+16$ & $2.55 \mathrm{E}-04$ \\
\hline $1.80 \mathrm{E}+01$ & 297.65 & $0.00 \mathrm{E}+00$ & $0.00 \mathrm{E}+00$ & $0.00 \mathrm{E}+00$ & $0.00 \mathrm{E}+00$ & $0.00 \mathrm{E}+00$ & $0.00 \mathrm{E}+00$ & $0.00 \mathrm{E}+00$ & $0.00 \mathrm{E}+00$ \\
\hline $1.90 \mathrm{E}+01$ & 307.26 & $0.00 \mathrm{E}+00$ & $0.00 \mathrm{E}+00$ & $0.00 \mathrm{E}+00$ & $0.00 \mathrm{E}+00$ & $0.00 \mathrm{E}+00$ & $0.00 \mathrm{E}+00$ & $0.00 \mathrm{E}+00$ & $0.00 \mathrm{E}+00$ \\
\hline \multirow[t]{2}{*}{$2.00 \mathrm{E}+01$} & 316.36 & $0.00 \mathrm{E}+00$ & $0.00 \mathrm{E}+00$ & $0.00 \mathrm{E}+00$ & $0.00 \mathrm{E}+00$ & $0.00 \mathrm{E}+00$ & $0.00 \mathrm{E}+00$ & $0.00 \mathrm{E}+00$ & $0.00 \mathrm{E}+00$ \\
\hline & & & $6.74 E+00$ & & $6.75 \mathrm{E}+00$ & & $6.72 E+00$ & & $6.65 E+00$ \\
\hline
\end{tabular}


Table 17. Calculated fluence and DPA for Capsule 4 of the UCSB experiment.

\begin{tabular}{|c|c|c|c|c|c|c|c|}
\hline \multirow{2}{*}{$\begin{array}{c}\text { Upper } \\
\text { Energy(MeV) }\end{array}$} & \multirow{2}{*}{$\begin{array}{l}\text { DPA xsec } \\
\text { keV-barn }\end{array}$} & \multicolumn{2}{|c|}{ 4-3 } & \multicolumn{2}{|c|}{ 4-2 } & \multicolumn{2}{|c|}{ 4-1 } \\
\hline & & Total Fluence & DPA & Total Fluence & DPA & Total Fluence & DPA \\
\hline $1.00 \mathrm{E}-10$ & 0 & $1.79 \mathrm{E}+15$ & & $3.10 \mathrm{E}+15$ & & $1.84 \mathrm{E}+15$ & \\
\hline $1.00 \mathrm{E}-09$ & 7.73 & $7.24 \mathrm{E}+17$ & 6.99E-05 & $6.02 \mathrm{E}+17$ & 5.81E-05 & $6.68 \mathrm{E}+17$ & $6.46 \mathrm{E}-05$ \\
\hline $1.00 \mathrm{E}-08$ & 2.444 & $1.19 \mathrm{E}+20$ & 3.62E-03 & $1.11 \mathrm{E}+20$ & $3.39 \mathrm{E}-03$ & $1.11 \mathrm{E}+20$ & $3.40 \mathrm{E}-03$ \\
\hline $2.30 \mathrm{E}-08$ & 1.278 & $4.26 \mathrm{E}+20$ & $6.81 \mathrm{E}-03$ & $4.06 \mathrm{E}+20$ & $6.49 \mathrm{E}-03$ & $4.02 E+20$ & $6.42 \mathrm{E}-03$ \\
\hline $5.00 \mathrm{E}-08$ & 0.857 & $1.06 \mathrm{E}+21$ & $1.13 \mathrm{E}-02$ & $1.01 \mathrm{E}+21$ & $1.08 \mathrm{E}-02$ & $9.86 \mathrm{E}+20$ & $1.06 \mathrm{E}-02$ \\
\hline $7.60 \mathrm{E}-08$ & 0.644 & $7.56 \mathrm{E}+20$ & $6.08 \mathrm{E}-03$ & $7.25 E+20$ & $5.84 \mathrm{E}-03$ & $7.08 \mathrm{E}+20$ & $5.70 \mathrm{E}-03$ \\
\hline $1.15 \mathrm{E}-07$ & 0.523 & $6.49 \mathrm{E}+20$ & $4.25 \mathrm{E}-03$ & $6.24 \mathrm{E}+20$ & $4.08 \mathrm{E}-03$ & $6.11 \mathrm{E}+20$ & 4.00E-03 \\
\hline $1.70 \mathrm{E}-07$ & 0.428 & $4.12 \mathrm{E}+20$ & $2.20 \mathrm{E}-03$ & $3.98 \mathrm{E}+20$ & $2.13 \mathrm{E}-03$ & $3.85 E+20$ & $2.06 \mathrm{E}-03$ \\
\hline $2.55 \mathrm{E}-07$ & 0.351 & $2.86 \mathrm{E}+20$ & $1.25 \mathrm{E}-03$ & $2.80 \mathrm{E}+20$ & $1.23 \mathrm{E}-03$ & $2.76 \mathrm{E}+20$ & $1.21 \mathrm{E}-03$ \\
\hline $3.80 \mathrm{E}-07$ & 0.287 & $2.41 E+20$ & 8.66E-04 & $2.30 \mathrm{E}+20$ & $8.25 \mathrm{E}-04$ & $2.22 \mathrm{E}+20$ & 7.98E-04 \\
\hline $5.50 \mathrm{E}-07$ & 0.237 & $2.19 \mathrm{E}+20$ & $6.50 \mathrm{E}-04$ & $2.14 \mathrm{E}+20$ & $6.33 \mathrm{E}-04$ & $2.06 \mathrm{E}+20$ & 6.10E-04 \\
\hline $8.40 \mathrm{E}-07$ & 0.194 & $2.43 \mathrm{E}+20$ & 5.89E-04 & $2.35 \mathrm{E}+20$ & 5.71E-04 & $2.28 \mathrm{E}+20$ & 5.53E-04 \\
\hline $1.28 \mathrm{E}-06$ & 0.157 & $2.30 \mathrm{E}+20$ & 4.51E-04 & $2.26 \mathrm{E}+20$ & 4.44E-04 & $2.21 \mathrm{E}+20$ & 4.33E-04 \\
\hline $1.90 \mathrm{E}-06$ & 0.128 & $2.16 \mathrm{E}+20$ & $3.45 \mathrm{E}-04$ & $2.11 \mathrm{E}+20$ & $3.38 \mathrm{E}-04$ & $2.06 \mathrm{E}+20$ & $3.30 \mathrm{E}-04$ \\
\hline $2.80 \mathrm{E}-06$ & 0.105 & $2.14 \mathrm{E}+20$ & $2.80 \mathrm{E}-04$ & $2.07 E+20$ & $2.72 \mathrm{E}-04$ & $1.97 \mathrm{E}+20$ & 2.59E-04 \\
\hline 4.25E-06 & 0.086 & $2.23 \mathrm{E}+20$ & $2.39 \mathrm{E}-04$ & $2.16 \mathrm{E}+20$ & $2.32 \mathrm{E}-04$ & $2.12 \mathrm{E}+20$ & $2.28 \mathrm{E}-04$ \\
\hline $6.30 \mathrm{E}-06$ & 0.07 & $2.11 \mathrm{E}+20$ & $1.85 \mathrm{E}-04$ & $2.05 \mathrm{E}+20$ & $1.80 \mathrm{E}-04$ & $1.99 \mathrm{E}+20$ & $1.74 \mathrm{E}-04$ \\
\hline $9.20 \mathrm{E}-06$ & 0.058 & $2.07 E+20$ & $1.50 \mathrm{E}-04$ & $1.98 \mathrm{E}+20$ & $1.43 \mathrm{E}-04$ & $1.92 \mathrm{E}+20$ & $1.39 \mathrm{E}-04$ \\
\hline $1.35 \mathrm{E}-05$ & 0.048 & $2.09 \mathrm{E}+20$ & $1.26 \mathrm{E}-04$ & $2.07 E+20$ & $1.24 \mathrm{E}-04$ & $1.96 \mathrm{E}+20$ & 1.17E-04 \\
\hline 2.10E-05 & 0.038 & $2.39 \mathrm{E}+20$ & $1.14 \mathrm{E}-04$ & $2.29 \mathrm{E}+20$ & $1.09 \mathrm{E}-04$ & $2.22 \mathrm{E}+20$ & $1.05 \mathrm{E}-04$ \\
\hline $3.00 \mathrm{E}-05$ & 0.031 & $1.98 \mathrm{E}+20$ & 7.66E-05 & $1.95 \mathrm{E}+20$ & 7.55E-05 & $1.87 \mathrm{E}+20$ & $7.23 \mathrm{E}-05$ \\
\hline $4.50 \mathrm{E}-05$ & 0.026 & $2.27 \mathrm{E}+20$ & 7.36E-05 & $2.25 \mathrm{E}+20$ & 7.33E-05 & $2.16 \mathrm{E}+20$ & 7.01E-05 \\
\hline $6.90 \mathrm{E}-05$ & 0.021 & $2.40 \mathrm{E}+20$ & $6.31 \mathrm{E}-05$ & $2.36 \mathrm{E}+20$ & 6.19E-05 & $2.34 \mathrm{E}+20$ & 6.15E-05 \\
\hline $1.00 \mathrm{E}-04$ & 0.017 & $2.14 \mathrm{E}+20$ & 4.55E-05 & $2.08 \mathrm{E}+20$ & 4.42E-05 & $2.08 \mathrm{E}+20$ & 4.43E-05 \\
\hline $1.35 \mathrm{E}-04$ & 0.014 & $1.75 \mathrm{E}+20$ & 3.07E-05 & $1.68 \mathrm{E}+20$ & $2.95 \mathrm{E}-05$ & $1.63 \mathrm{E}+20$ & $2.85 \mathrm{E}-05$ \\
\hline $1.70 \mathrm{E}-04$ & 0.014 & $1.38 \mathrm{E}+20$ & $2.42 \mathrm{E}-05$ & $1.30 \mathrm{E}+20$ & 2.27E-05 & $1.27 \mathrm{E}+20$ & $2.23 \mathrm{E}-05$ \\
\hline $2.20 \mathrm{E}-04$ & 0.011 & $1.50 \mathrm{E}+20$ & 2.06E-05 & $1.46 \mathrm{E}+20$ & $2.01 \mathrm{E}-05$ & $1.43 \mathrm{E}+20$ & $1.97 \mathrm{E}-05$ \\
\hline
\end{tabular}




\begin{tabular}{|c|c|c|c|c|c|c|c|}
\hline \multirow{2}{*}{$\begin{array}{c}\text { Upper } \\
\text { Energy(MeV) }\end{array}$} & \multirow{2}{*}{$\begin{array}{l}\text { DPA xsec } \\
\text { keV-barn }\end{array}$} & \multicolumn{2}{|c|}{$4-3$} & \multicolumn{2}{|c|}{ 4-2 } & \multicolumn{2}{|c|}{ 4-1 } \\
\hline & & Total Fluence & DPA & Total Fluence & DPA & Total Fluence & DPA \\
\hline $2.80 \mathrm{E}-04$ & 0.011 & $1.46 \mathrm{E}+20$ & $2.00 \mathrm{E}-05$ & $1.40 \mathrm{E}+20$ & $1.93 \mathrm{E}-05$ & $1.34 \mathrm{E}+20$ & $1.84 \mathrm{E}-05$ \\
\hline $3.60 E-04$ & 0.01 & $1.50 \mathrm{E}+20$ & $1.88 \mathrm{E}-05$ & $1.41 \mathrm{E}+20$ & $1.76 \mathrm{E}-05$ & $1.40 \mathrm{E}+20$ & $1.76 \mathrm{E}-05$ \\
\hline $4.50 \mathrm{E}-04$ & 0.007 & $1.29 \mathrm{E}+20$ & $1.13 \mathrm{E}-05$ & $1.26 \mathrm{E}+20$ & $1.10 \mathrm{E}-05$ & $1.22 \mathrm{E}+20$ & 1.07E-05 \\
\hline $5.75 \mathrm{E}-04$ & 0.005 & $1.45 \mathrm{E}+20$ & $9.04 \mathrm{E}-06$ & $1.41 \mathrm{E}+20$ & $8.81 \mathrm{E}-06$ & $1.36 \mathrm{E}+20$ & 8.49E-06 \\
\hline $7.60 \mathrm{E}-04$ & 0.129 & $1.64 \mathrm{E}+20$ & $2.64 \mathrm{E}-04$ & $1.58 \mathrm{E}+20$ & $2.54 \mathrm{E}-04$ & $1.54 \mathrm{E}+20$ & $2.48 \mathrm{E}-04$ \\
\hline $9.60 \mathrm{E}-04$ & 0.308 & $1.37 E+20$ & $5.28 \mathrm{E}-04$ & $1.34 \mathrm{E}+20$ & $5.16 \mathrm{E}-04$ & $1.31 \mathrm{E}+20$ & 5.04E-04 \\
\hline $1.28 \mathrm{E}-03$ & 0.672 & $1.67 \mathrm{E}+20$ & $1.41 \mathrm{E}-03$ & $1.61 \mathrm{E}+20$ & $1.35 \mathrm{E}-03$ & $1.59 \mathrm{E}+20$ & $1.34 \mathrm{E}-03$ \\
\hline $1.60 \mathrm{E}-03$ & 0.477 & $1.35 \mathrm{E}+20$ & 8.06E-04 & $1.29 \mathrm{E}+20$ & $7.68 \mathrm{E}-04$ & $1.28 \mathrm{E}+20$ & 7.62E-04 \\
\hline $2.00 \mathrm{E}-03$ & 0.511 & $1.39 \mathrm{E}+20$ & 8.91E-04 & $1.34 \mathrm{E}+20$ & $8.56 \mathrm{E}-04$ & $1.30 \mathrm{E}+20$ & $8.32 \mathrm{E}-04$ \\
\hline $2.70 \mathrm{E}-03$ & 0.568 & $1.79 \mathrm{E}+20$ & 1.27E-03 & $1.78 \mathrm{E}+20$ & $1.27 \mathrm{E}-03$ & $1.68 \mathrm{E}+20$ & 1.20E-03 \\
\hline $3.40 \mathrm{E}-03$ & 0.632 & $1.45 \mathrm{E}+20$ & $1.14 \mathrm{E}-03$ & $1.37 \mathrm{E}+20$ & $1.08 \mathrm{E}-03$ & $1.38 \mathrm{E}+20$ & $1.09 \mathrm{E}-03$ \\
\hline $4.50 \mathrm{E}-03$ & 0.822 & $1.78 \mathrm{E}+20$ & $1.83 \mathrm{E}-03$ & $1.67 \mathrm{E}+20$ & $1.72 \mathrm{E}-03$ & $1.67 E+20$ & $1.72 \mathrm{E}-03$ \\
\hline $5.50 \mathrm{E}-03$ & 0.826 & $1.28 \mathrm{E}+20$ & $1.32 \mathrm{E}-03$ & $1.28 \mathrm{E}+20$ & $1.32 \mathrm{E}-03$ & $1.25 \mathrm{E}+20$ & $1.29 \mathrm{E}-03$ \\
\hline 7.20E-03 & 1.671 & $1.69 \mathrm{E}+20$ & 3.53E-03 & $1.69 \mathrm{E}+20$ & $3.53 \mathrm{E}-03$ & $1.61 \mathrm{E}+20$ & 3.37E-03 \\
\hline $9.20 \mathrm{E}-03$ & 3.709 & $1.40 \mathrm{E}+20$ & $6.49 \mathrm{E}-03$ & $1.38 \mathrm{E}+20$ & 6.39E-03 & $1.39 \mathrm{E}+20$ & $6.45 \mathrm{E}-03$ \\
\hline $1.20 \mathrm{E}-02$ & 1.491 & $1.71 \mathrm{E}+20$ & $3.18 \mathrm{E}-03$ & $1.71 \mathrm{E}+20$ & $3.18 \mathrm{E}-03$ & $1.54 \mathrm{E}+20$ & $2.87 \mathrm{E}-03$ \\
\hline $1.50 \mathrm{E}-02$ & 1.156 & $1.51 \mathrm{E}+20$ & $2.18 \mathrm{E}-03$ & $1.49 \mathrm{E}+20$ & $2.15 \mathrm{E}-03$ & $1.40 \mathrm{E}+20$ & $2.03 \mathrm{E}-03$ \\
\hline $1.90 \mathrm{E}-02$ & 0.973 & $1.44 \mathrm{E}+20$ & $1.75 \mathrm{E}-03$ & $1.41 \mathrm{E}+20$ & $1.71 \mathrm{E}-03$ & $1.50 \mathrm{E}+20$ & $1.82 \mathrm{E}-03$ \\
\hline $2.55 \mathrm{E}-02$ & 0.555 & $2.22 \mathrm{E}+20$ & $1.54 \mathrm{E}-03$ & $2.22 \mathrm{E}+20$ & $1.54 \mathrm{E}-03$ & $2.16 \mathrm{E}+20$ & $1.50 \mathrm{E}-03$ \\
\hline $3.20 \mathrm{E}-02$ & 26.762 & $2.02 \mathrm{E}+20$ & $6.76 \mathrm{E}-02$ & $1.95 \mathrm{E}+20$ & $6.52 \mathrm{E}-02$ & $1.95 \mathrm{E}+20$ & $6.52 \mathrm{E}-02$ \\
\hline $4.00 \mathrm{E}-02$ & 8.161 & $9.97 \mathrm{E}+19$ & $1.02 \mathrm{E}-02$ & $9.74 \mathrm{E}+19$ & $9.93 \mathrm{E}-03$ & $9.16 \mathrm{E}+19$ & $9.34 \mathrm{E}-03$ \\
\hline $5.25 \mathrm{E}-02$ & 6.515 & $1.99 \mathrm{E}+20$ & $1.62 \mathrm{E}-02$ & $1.96 \mathrm{E}+20$ & $1.59 \mathrm{E}-02$ & $1.91 \mathrm{E}+20$ & $1.55 \mathrm{E}-02$ \\
\hline $6.60 \mathrm{E}-02$ & 6.576 & $1.99 \mathrm{E}+20$ & $1.64 \mathrm{E}-02$ & $1.94 \mathrm{E}+20$ & $1.59 \mathrm{E}-02$ & $1.85 \mathrm{E}+20$ & $1.52 \mathrm{E}-02$ \\
\hline $8.80 \mathrm{E}-02$ & 13.137 & $2.85 E+20$ & 4.69E-02 & $2.84 \mathrm{E}+20$ & 4.66E-02 & $2.76 \mathrm{E}+20$ & $4.53 \mathrm{E}-02$ \\
\hline 1.10E-01 & 11.84 & $1.80 \mathrm{E}+20$ & 2.67E-02 & $1.75 \mathrm{E}+20$ & $2.59 \mathrm{E}-02$ & $1.72 \mathrm{E}+20$ & $2.54 \mathrm{E}-02$ \\
\hline $1.35 \mathrm{E}-01$ & 11.27 & $2.64 \mathrm{E}+20$ & 3.72E-02 & $2.59 \mathrm{E}+20$ & $3.65 \mathrm{E}-02$ & $2.47 \mathrm{E}+20$ & 3.47E-02 \\
\hline $1.60 \mathrm{E}-01$ & 21.53 & $1.60 \mathrm{E}+20$ & 4.30E-02 & $1.48 \mathrm{E}+20$ & $4.00 \mathrm{E}-02$ & $1.51 \mathrm{E}+20$ & 4.07E-02 \\
\hline $1.90 \mathrm{E}-01$ & 15.85 & $2.10 \mathrm{E}+20$ & 4.16E-02 & $2.06 \mathrm{E}+20$ & $4.08 \mathrm{E}-02$ & $2.02 \mathrm{E}+20$ & 3.99E-02 \\
\hline $2.20 \mathrm{E}-01$ & 22.01 & $1.74 \mathrm{E}+20$ & 4.79E-02 & $1.68 \mathrm{E}+20$ & $4.62 \mathrm{E}-02$ & $1.66 \mathrm{E}+20$ & $4.56 \mathrm{E}-02$ \\
\hline
\end{tabular}




\begin{tabular}{|c|c|c|c|c|c|c|c|}
\hline \multirow{2}{*}{$\begin{array}{c}\text { Upper } \\
\text { Energy(MeV) }\end{array}$} & \multirow{2}{*}{$\begin{array}{l}\text { DPA xsec } \\
\text { keV-barn }\end{array}$} & \multicolumn{2}{|c|}{$4-3$} & \multicolumn{2}{|c|}{ 4-2 } & \multicolumn{2}{|c|}{ 4-1 } \\
\hline & & Total Fluence & DPA & Total Fluence & DPA & Total Fluence & DPA \\
\hline $2.55 \mathrm{E}-01$ & 18.27 & $2.02 \mathrm{E}+20$ & 4.61E-02 & $1.98 \mathrm{E}+20$ & $4.51 \mathrm{E}-02$ & $1.96 \mathrm{E}+20$ & $4.48 \mathrm{E}-02$ \\
\hline $2.90 \mathrm{E}-01$ & 17.37 & $2.22 \mathrm{E}+20$ & $4.82 \mathrm{E}-02$ & $2.14 \mathrm{E}+20$ & 4.64E-02 & $2.09 \mathrm{E}+20$ & 4.54E-02 \\
\hline $3.20 \mathrm{E}-01$ & 13.51 & $1.53 \mathrm{E}+20$ & $2.58 \mathrm{E}-02$ & $1.49 \mathrm{E}+20$ & $2.51 \mathrm{E}-02$ & $1.44 \mathrm{E}+20$ & $2.43 \mathrm{E}-02$ \\
\hline $3.60 \mathrm{E}-01$ & 25.14 & $2.21 \mathrm{E}+20$ & $6.94 \mathrm{E}-02$ & $2.14 \mathrm{E}+20$ & $6.73 \mathrm{E}-02$ & $2.06 \mathrm{E}+20$ & $6.48 \mathrm{E}-02$ \\
\hline 4.00E-01 & 46.52 & $1.90 \mathrm{E}+20$ & $1.11 \mathrm{E}-01$ & $1.82 \mathrm{E}+20$ & $1.06 \mathrm{E}-01$ & $1.79 \mathrm{E}+20$ & $1.04 \mathrm{E}-01$ \\
\hline $4.50 \mathrm{E}-01$ & 43.04 & $1.78 \mathrm{E}+20$ & $9.59 \mathrm{E}-02$ & $1.74 \mathrm{E}+20$ & 9.37E-02 & $1.67 \mathrm{E}+20$ & 8.97E-02 \\
\hline $5.00 \mathrm{E}-01$ & 36.4 & $2.01 \mathrm{E}+20$ & $9.15 \mathrm{E}-02$ & $1.92 \mathrm{E}+20$ & $8.75 \mathrm{E}-02$ & $1.89 \mathrm{E}+20$ & $8.61 \mathrm{E}-02$ \\
\hline $5.50 \mathrm{E}-01$ & 33.22 & $1.99 \mathrm{E}+20$ & 8.27E-02 & $1.91 \mathrm{E}+20$ & $7.92 \mathrm{E}-02$ & $1.91 \mathrm{E}+20$ & 7.94E-02 \\
\hline $6.00 \mathrm{E}-01$ & 34.18 & $1.91 \mathrm{E}+20$ & 8.15E-02 & $1.85 \mathrm{E}+20$ & 7.91E-02 & $1.82 \mathrm{E}+20$ & 7.78E-02 \\
\hline $6.60 \mathrm{E}-01$ & 19.64 & $2.31 E+20$ & 5.67E-02 & $2.27 E+20$ & $5.58 \mathrm{E}-02$ & $2.21 \mathrm{E}+20$ & $5.42 \mathrm{E}-02$ \\
\hline 7.20E-01 & 48.89 & $2.42 \mathrm{E}+20$ & $1.48 \mathrm{E}-01$ & $2.33 \mathrm{E}+20$ & $1.42 \mathrm{E}-01$ & $2.32 \mathrm{E}+20$ & $1.42 \mathrm{E}-01$ \\
\hline $7.80 \mathrm{E}-01$ & 74.22 & $2.30 \mathrm{E}+20$ & $2.14 \mathrm{E}-01$ & $2.19 E+20$ & $2.04 \mathrm{E}-01$ & $2.23 E+20$ & 2.07E-01 \\
\hline $8.40 \mathrm{E}-01$ & 44.02 & $1.81 \mathrm{E}+20$ & $9.98 \mathrm{E}-02$ & $1.75 \mathrm{E}+20$ & $9.62 \mathrm{E}-02$ & $1.71 \mathrm{E}+20$ & $9.41 \mathrm{E}-02$ \\
\hline $9.20 \mathrm{E}-01$ & 40.97 & $2.20 \mathrm{E}+20$ & 1.13E-01 & $2.05 E+20$ & $1.05 \mathrm{E}-01$ & $2.02 \mathrm{E}+20$ & $1.04 \mathrm{E}-01$ \\
\hline $1.00 \mathrm{E}+00$ & 50.95 & $1.99 \mathrm{E}+20$ & 1.27E-01 & $1.93 \mathrm{E}+20$ & $1.23 \mathrm{E}-01$ & $1.92 \mathrm{E}+20$ & $1.22 \mathrm{E}-01$ \\
\hline $1.20 \mathrm{E}+00$ & 50.09 & $4.69 \mathrm{E}+20$ & $2.94 \mathrm{E}-01$ & $4.57 \mathrm{E}+20$ & $2.86 \mathrm{E}-01$ & $4.39 \mathrm{E}+20$ & $2.75 \mathrm{E}-01$ \\
\hline $1.40 \mathrm{E}+00$ & 64.54 & $4.10 \mathrm{E}+20$ & 3.31E-01 & $4.05 E+20$ & $3.27 \mathrm{E}-01$ & $3.98 \mathrm{E}+20$ & 3.21E-01 \\
\hline $1.60 \mathrm{E}+00$ & 73.58 & $3.55 \mathrm{E}+20$ & $3.27 \mathrm{E}-01$ & $3.53 \mathrm{E}+20$ & $3.25 \mathrm{E}-01$ & $3.48 \mathrm{E}+20$ & $3.20 \mathrm{E}-01$ \\
\hline $1.80 \mathrm{E}+00$ & 76.46 & $3.10 \mathrm{E}+20$ & 2.96E-01 & $3.03 E+20$ & $2.89 \mathrm{E}-01$ & $2.93 \mathrm{E}+20$ & $2.80 \mathrm{E}-01$ \\
\hline $2.00 \mathrm{E}+00$ & 95.15 & $2.76 \mathrm{E}+20$ & $3.28 \mathrm{E}-01$ & $2.67 E+20$ & 3.17E-01 & $2.61 E+20$ & 3.10E-01 \\
\hline $2.30 \mathrm{E}+00$ & 93.75 & $3.37 \mathrm{E}+20$ & 3.95E-01 & $3.35 \mathrm{E}+20$ & $3.92 \mathrm{E}-01$ & $3.22 \mathrm{E}+20$ & 3.77E-01 \\
\hline $2.60 \mathrm{E}+00$ & 112.05 & $3.12 \mathrm{E}+20$ & 4.37E-01 & $3.07 E+20$ & $4.30 \mathrm{E}-01$ & $2.98 \mathrm{E}+20$ & $4.18 \mathrm{E}-01$ \\
\hline $2.90 \mathrm{E}+00$ & 123.55 & $2.38 \mathrm{E}+20$ & $3.68 \mathrm{E}-01$ & $2.32 \mathrm{E}+20$ & $3.58 \mathrm{E}-01$ & $2.26 \mathrm{E}+20$ & $3.49 \mathrm{E}-01$ \\
\hline $3.30 \mathrm{E}+00$ & 133.45 & $2.36 \mathrm{E}+20$ & 3.93E-01 & $2.30 \mathrm{E}+20$ & 3.84E-01 & $2.26 \mathrm{E}+20$ & 3.77E-01 \\
\hline $3.70 \mathrm{E}+00$ & 135.25 & $1.60 \mathrm{E}+20$ & 2.70E-01 & $1.57 \mathrm{E}+20$ & $2.66 \mathrm{E}-01$ & $1.51 \mathrm{E}+20$ & $2.56 \mathrm{E}-01$ \\
\hline $4.10 \mathrm{E}+00$ & 149.55 & $1.24 \mathrm{E}+20$ & 2.31E-01 & $1.19 \mathrm{E}+20$ & $2.22 \mathrm{E}-01$ & $1.20 \mathrm{E}+20$ & $2.25 \mathrm{E}-01$ \\
\hline $4.50 \mathrm{E}+00$ & 158.25 & $9.59 \mathrm{E}+19$ & $1.90 \mathrm{E}-01$ & $9.26 \mathrm{E}+19$ & $1.83 \mathrm{E}-01$ & $9.07 E+19$ & $1.79 \mathrm{E}-01$ \\
\hline $5.00 \mathrm{E}+00$ & 168.55 & $8.77 \mathrm{E}+19$ & $1.85 \mathrm{E}-01$ & $8.83 E+19$ & $1.86 \mathrm{E}-01$ & $8.46 \mathrm{E}+19$ & $1.78 \mathrm{E}-01$ \\
\hline $5.50 \mathrm{E}+00$ & 176.46 & $6.23 E+19$ & 1.37E-01 & $6.16 \mathrm{E}+19$ & $1.36 \mathrm{E}-01$ & $5.78 \mathrm{E}+19$ & $1.28 \mathrm{E}-01$ \\
\hline
\end{tabular}




\begin{tabular}{|c|c|c|c|c|c|c|c|}
\hline \multirow{2}{*}{$\begin{array}{c}\text { Upper } \\
\text { Energy(MeV) }\end{array}$} & \multirow{2}{*}{$\begin{array}{l}\text { DPA xsec } \\
\text { keV-barn }\end{array}$} & \multicolumn{2}{|c|}{ 4-3 } & \multicolumn{2}{|c|}{$4-2$} & \multicolumn{2}{|c|}{ 4-1 } \\
\hline & & Total Fluence & DPA & Total Fluence & DPA & Total Fluence & DPA \\
\hline $6.00 \mathrm{E}+00$ & 183.06 & $4.44 \mathrm{E}+19$ & $1.01 \mathrm{E}-01$ & $4.34 \mathrm{E}+19$ & $9.93 \mathrm{E}-02$ & $4.19 E+19$ & 9.59E-02 \\
\hline $6.70 \mathrm{E}+00$ & 189.26 & $3.89 E+19$ & $9.20 \mathrm{E}-02$ & $4.03 E+19$ & $9.54 \mathrm{E}-02$ & $3.63 E+19$ & 8.59E-02 \\
\hline $7.40 \mathrm{E}+00$ & 196.67 & $2.23 \mathrm{E}+19$ & $5.49 \mathrm{E}-02$ & $2.12 \mathrm{E}+19$ & $5.21 \mathrm{E}-02$ & $2.16 \mathrm{E}+19$ & 5.30E-02 \\
\hline $8.20 \mathrm{E}+00$ & 203.37 & $1.50 \mathrm{E}+19$ & $3.81 \mathrm{E}-02$ & $1.42 \mathrm{E}+19$ & $3.60 \mathrm{E}-02$ & $1.26 \mathrm{E}+19$ & 3.19E-02 \\
\hline $9.00 \mathrm{E}+00$ & 214.58 & $8.55 \mathrm{E}+18$ & $2.29 \mathrm{E}-02$ & $7.15 \mathrm{E}+18$ & $1.92 \mathrm{E}-02$ & $8.44 \mathrm{E}+18$ & $2.26 \mathrm{E}-02$ \\
\hline $1.00 \mathrm{E}+01$ & 225.69 & $5.20 \mathrm{E}+18$ & $1.47 \mathrm{E}-02$ & $4.84 \mathrm{E}+18$ & $1.36 \mathrm{E}-02$ & $4.42 \mathrm{E}+18$ & $1.25 \mathrm{E}-02$ \\
\hline $1.10 \mathrm{E}+01$ & 237.6 & $1.95 \mathrm{E}+18$ & $5.80 \mathrm{E}-03$ & $2.17 \mathrm{E}+18$ & $6.44 \mathrm{E}-03$ & $2.66 \mathrm{E}+18$ & 7.89E-03 \\
\hline $1.20 \mathrm{E}+01$ & 247.41 & $1.12 \mathrm{E}+18$ & 3.47E-03 & $9.82 \mathrm{E}+17$ & $3.04 \mathrm{E}-03$ & $8.23 \mathrm{E}+17$ & $2.55 \mathrm{E}-03$ \\
\hline $1.30 \mathrm{E}+01$ & 258.52 & $5.90 \mathrm{E}+17$ & $1.91 \mathrm{E}-03$ & $3.84 \mathrm{E}+17$ & $1.24 \mathrm{E}-03$ & $5.30 \mathrm{E}+17$ & $1.71 \mathrm{E}-03$ \\
\hline $1.40 \mathrm{E}+01$ & 271.35 & $3.73 \mathrm{E}+17$ & $1.26 \mathrm{E}-03$ & $1.59 \mathrm{E}+17$ & 5.39E-04 & 1.17E+17 & $3.98 \mathrm{E}-04$ \\
\hline $1.50 \mathrm{E}+01$ & 290.27 & $4.42 \mathrm{E}+16$ & $1.61 \mathrm{E}-04$ & $8.78 \mathrm{E}+16$ & 3.19E-04 & $2.79 \mathrm{E}+16$ & $1.01 \mathrm{E}-04$ \\
\hline $1.60 \mathrm{E}+01$ & 293.2 & $3.68 \mathrm{E}+16$ & $1.35 \mathrm{E}-04$ & $3.89 \mathrm{E}+16$ & $1.43 \mathrm{E}-04$ & $3.91 \mathrm{E}+16$ & $1.43 \mathrm{E}-04$ \\
\hline $1.70 \mathrm{E}+01$ & 292.73 & $0.00 \mathrm{E}+00$ & $0.00 \mathrm{E}+00$ & $0.00 \mathrm{E}+00$ & $0.00 \mathrm{E}+00$ & $0.00 \mathrm{E}+00$ & $0.00 \mathrm{E}+00$ \\
\hline $1.80 \mathrm{E}+01$ & 297.65 & $0.00 \mathrm{E}+00$ & $0.00 \mathrm{E}+00$ & $6.02 \mathrm{E}+15$ & $2.24 \mathrm{E}-05$ & $1.59 \mathrm{E}+16$ & $5.91 \mathrm{E}-05$ \\
\hline $1.90 \mathrm{E}+01$ & 307.26 & $0.00 \mathrm{E}+00$ & $0.00 \mathrm{E}+00$ & $0.00 \mathrm{E}+00$ & $0.00 E+00$ & $0.00 \mathrm{E}+00$ & $0.00 \mathrm{E}+00$ \\
\hline \multirow[t]{2}{*}{$2.00 \mathrm{E}+01$} & 316.36 & $0.00 \mathrm{E}+00$ & $0.00 \mathrm{E}+00$ & $0.00 \mathrm{E}+00$ & $0.00 \mathrm{E}+00$ & $0.00 \mathrm{E}+00$ & $0.00 \mathrm{E}+00$ \\
\hline & & & $6.35 E+00$ & & $6.19 E+00$ & & $6.05 E+00$ \\
\hline
\end{tabular}


Table 18. Calculated fluence and DPA for capsule 3 of the UCSB experiment.

\begin{tabular}{|c|c|c|c|c|c|c|c|c|c|}
\hline \multirow{2}{*}{$\begin{array}{c}\text { Upper } \\
\text { Energy(MeV) }\end{array}$} & \multirow{2}{*}{$\begin{array}{l}\text { DPA xsec } \\
\text { keV-barn }\end{array}$} & \multicolumn{2}{|c|}{$3-4$} & \multicolumn{2}{|c|}{$3-3$} & \multicolumn{2}{|c|}{$3-2$} & \multicolumn{2}{|c|}{ 3-1 } \\
\hline & & Total Fluence & DPA & Total Fluence & DPA & Total Fluence & DPA & Total Fluence & DPA \\
\hline $1.00 \mathrm{E}-10$ & 0 & $2.38 \mathrm{E}+15$ & & $5.96 \mathrm{E}+15$ & & $2.33 \mathrm{E}+15$ & & $1.03 E+16$ & \\
\hline $1.00 \mathrm{E}-09$ & 7.73 & $5.98 \mathrm{E}+17$ & 5.77E-05 & $5.43 E+17$ & $5.25 \mathrm{E}-05$ & $6.02 E+17$ & 5.81E-05 & $3.23 E+17$ & $3.12 \mathrm{E}-05$ \\
\hline $1.00 \mathrm{E}-08$ & 2.444 & $9.77 \mathrm{E}+19$ & $2.98 \mathrm{E}-03$ & $9.01 E+19$ & $2.75 \mathrm{E}-03$ & $9.04 \mathrm{E}+19$ & 2.76E-03 & $8.37 E+19$ & $2.56 \mathrm{E}-03$ \\
\hline $2.30 \mathrm{E}-08$ & 1.278 & $3.56 \mathrm{E}+20$ & $5.69 \mathrm{E}-03$ & $3.33 E+20$ & $5.32 \mathrm{E}-03$ & $3.13 E+20$ & $5.00 \mathrm{E}-03$ & $3.08 \mathrm{E}+20$ & $4.92 \mathrm{E}-03$ \\
\hline $5.00 \mathrm{E}-08$ & 0.857 & $8.85 E+20$ & $9.48 \mathrm{E}-03$ & $8.18 \mathrm{E}+20$ & $8.76 \mathrm{E}-03$ & $7.91 E+20$ & $8.48 \mathrm{E}-03$ & $7.65 E+20$ & $8.20 \mathrm{E}-03$ \\
\hline $7.60 \mathrm{E}-08$ & 0.644 & $6.35 \mathrm{E}+20$ & 5.11E-03 & $5.85 \mathrm{E}+20$ & 4.71E-03 & $5.64 \mathrm{E}+20$ & 4.54E-03 & $5.42 \mathrm{E}+20$ & 4.36E-03 \\
\hline $1.15 \mathrm{E}-07$ & 0.523 & $5.52 \mathrm{E}+20$ & $3.61 \mathrm{E}-03$ & $5.13 \mathrm{E}+20$ & $3.35 \mathrm{E}-03$ & $4.91 E+20$ & $3.21 \mathrm{E}-03$ & $4.79 \mathrm{E}+20$ & $3.13 \mathrm{E}-03$ \\
\hline $1.70 \mathrm{E}-07$ & 0.428 & $3.48 \mathrm{E}+20$ & $1.86 \mathrm{E}-03$ & $3.26 \mathrm{E}+20$ & $1.74 \mathrm{E}-03$ & $3.12 \mathrm{E}+20$ & 1.67E-03 & $3.02 E+20$ & $1.61 \mathrm{E}-03$ \\
\hline $2.55 \mathrm{E}-07$ & 0.351 & $2.55 \mathrm{E}+20$ & $1.12 \mathrm{E}-03$ & $2.31 E+20$ & $1.02 \mathrm{E}-03$ & $2.21 E+20$ & $9.71 \mathrm{E}-04$ & $2.18 \mathrm{E}+20$ & 9.57E-04 \\
\hline $3.80 \mathrm{E}-07$ & 0.287 & $2.08 \mathrm{E}+20$ & $7.48 \mathrm{E}-04$ & $1.92 \mathrm{E}+20$ & $6.88 \mathrm{E}-04$ & $1.88 \mathrm{E}+20$ & $6.73 \mathrm{E}-04$ & $1.83 \mathrm{E}+20$ & $6.55 \mathrm{E}-04$ \\
\hline $5.50 \mathrm{E}-07$ & 0.237 & $1.90 \mathrm{E}+20$ & 5.63E-04 & $1.77 \mathrm{E}+20$ & $5.25 \mathrm{E}-04$ & $1.72 \mathrm{E}+20$ & 5.09E-04 & $1.65 \mathrm{E}+20$ & $4.90 \mathrm{E}-04$ \\
\hline $8.40 \mathrm{E}-07$ & 0.194 & $2.13 \mathrm{E}+20$ & $5.16 \mathrm{E}-04$ & $1.99 \mathrm{E}+20$ & 4.83E-04 & $1.95 \mathrm{E}+20$ & 4.73E-04 & $1.92 \mathrm{E}+20$ & 4.65E-04 \\
\hline $1.28 \mathrm{E}-06$ & 0.157 & $2.04 \mathrm{E}+20$ & 4.00E-04 & $1.96 \mathrm{E}+20$ & $3.84 \mathrm{E}-04$ & $1.85 \mathrm{E}+20$ & 3.64E-04 & $1.82 \mathrm{E}+20$ & $3.58 \mathrm{E}-04$ \\
\hline $1.90 \mathrm{E}-06$ & 0.128 & $1.92 \mathrm{E}+20$ & 3.07E-04 & $1.80 \mathrm{E}+20$ & $2.88 \mathrm{E}-04$ & $1.71 \mathrm{E}+20$ & $2.74 \mathrm{E}-04$ & $1.69 \mathrm{E}+20$ & $2.70 \mathrm{E}-04$ \\
\hline $2.80 \mathrm{E}-06$ & 0.105 & $1.88 \mathrm{E}+20$ & $2.46 \mathrm{E}-04$ & $1.78 \mathrm{E}+20$ & $2.33 \mathrm{E}-04$ & $1.69 \mathrm{E}+20$ & $2.22 \mathrm{E}-04$ & $1.61 \mathrm{E}+20$ & $2.11 \mathrm{E}-04$ \\
\hline 4.25E-06 & 0.086 & $1.98 \mathrm{E}+20$ & $2.13 \mathrm{E}-04$ & $1.87 \mathrm{E}+20$ & 2.01E-04 & $1.74 \mathrm{E}+20$ & 1.87E-04 & $1.52 \mathrm{E}+20$ & $1.63 \mathrm{E}-04$ \\
\hline $6.30 \mathrm{E}-06$ & 0.07 & $1.85 \mathrm{E}+20$ & $1.62 \mathrm{E}-04$ & $1.74 \mathrm{E}+20$ & $1.52 \mathrm{E}-04$ & $1.68 \mathrm{E}+20$ & 1.47E-04 & $1.64 \mathrm{E}+20$ & $1.43 \mathrm{E}-04$ \\
\hline $9.20 \mathrm{E}-06$ & 0.058 & $1.80 \mathrm{E}+20$ & 1.30E-04 & $1.64 \mathrm{E}+20$ & 1.19E-04 & $1.67 \mathrm{E}+20$ & $1.21 \mathrm{E}-04$ & $1.55 \mathrm{E}+20$ & $1.13 \mathrm{E}-04$ \\
\hline $1.35 \mathrm{E}-05$ & 0.048 & $1.87 \mathrm{E}+20$ & $1.12 \mathrm{E}-04$ & $1.79 \mathrm{E}+20$ & 1.07E-04 & $1.71 \mathrm{E}+20$ & $1.02 \mathrm{E}-04$ & $1.62 \mathrm{E}+20$ & $9.71 \mathrm{E}-05$ \\
\hline $2.10 \mathrm{E}-05$ & 0.038 & $2.11 \mathrm{E}+20$ & $1.00 \mathrm{E}-04$ & $2.02 \mathrm{E}+20$ & $9.60 \mathrm{E}-05$ & $1.86 \mathrm{E}+20$ & 8.83E-05 & $1.48 \mathrm{E}+20$ & 7.03E-05 \\
\hline $3.00 \mathrm{E}-05$ & 0.031 & $1.77 \mathrm{E}+20$ & $6.86 \mathrm{E}-05$ & $1.69 \mathrm{E}+20$ & $6.55 \mathrm{E}-05$ & $1.60 \mathrm{E}+20$ & $6.21 \mathrm{E}-05$ & $1.34 \mathrm{E}+20$ & $5.20 \mathrm{E}-05$ \\
\hline 4.50E-05 & 0.026 & $2.02 \mathrm{E}+20$ & $6.57 \mathrm{E}-05$ & $1.90 \mathrm{E}+20$ & $6.18 \mathrm{E}-05$ & $1.84 \mathrm{E}+20$ & 5.97E-05 & $1.75 \mathrm{E}+20$ & $5.70 \mathrm{E}-05$ \\
\hline 6.90E-05 & 0.021 & $2.15 \mathrm{E}+20$ & 5.64E-05 & $2.05 \mathrm{E}+20$ & 5.38E-05 & $1.91 \mathrm{E}+20$ & $5.02 \mathrm{E}-05$ & $1.80 \mathrm{E}+20$ & 4.72E-05 \\
\hline $1.00 \mathrm{E}-04$ & 0.017 & $1.95 \mathrm{E}+20$ & 4.14E-05 & $1.76 \mathrm{E}+20$ & 3.73E-05 & $1.68 \mathrm{E}+20$ & $3.58 \mathrm{E}-05$ & $1.66 \mathrm{E}+20$ & $3.52 \mathrm{E}-05$ \\
\hline $1.35 \mathrm{E}-04$ & 0.014 & $1.56 \mathrm{E}+20$ & $2.73 \mathrm{E}-05$ & $1.50 \mathrm{E}+20$ & $2.62 \mathrm{E}-05$ & $1.38 \mathrm{E}+20$ & $2.41 \mathrm{E}-05$ & $1.30 \mathrm{E}+20$ & $2.28 \mathrm{E}-05$ \\
\hline 1.70E-04 & 0.014 & $1.19 \mathrm{E}+20$ & $2.08 \mathrm{E}-05$ & $1.14 \mathrm{E}+20$ & $1.99 \mathrm{E}-05$ & $1.13 \mathrm{E}+20$ & $1.98 \mathrm{E}-05$ & $1.01 \mathrm{E}+20$ & $1.77 \mathrm{E}-05$ \\
\hline $2.20 \mathrm{E}-04$ & 0.011 & $1.33 \mathrm{E}+20$ & $1.83 \mathrm{E}-05$ & $1.31 \mathrm{E}+20$ & $1.81 \mathrm{E}-05$ & $1.25 \mathrm{E}+20$ & $1.72 \mathrm{E}-05$ & $1.18 \mathrm{E}+20$ & $1.62 \mathrm{E}-05$ \\
\hline
\end{tabular}




\begin{tabular}{|c|c|c|c|c|c|c|c|c|c|}
\hline \multirow[b]{2}{*}{$\begin{array}{c}\text { Upper } \\
\text { Energy(MeV) } \\
\end{array}$} & \multirow{2}{*}{$\begin{array}{l}\text { DPA xsec } \\
\text { keV-barn }\end{array}$} & \multicolumn{2}{|c|}{$3-4$} & \multicolumn{2}{|c|}{ 3-3 } & \multicolumn{2}{|c|}{$3-2$} & \multicolumn{2}{|c|}{ 3-1 } \\
\hline & & Total Fluence & DPA & Total Fluence & DPA & Total Fluence & DPA & Total Fluence & DPA \\
\hline $2.80 \mathrm{E}-04$ & 0.011 & $1.30 \mathrm{E}+20$ & $1.79 \mathrm{E}-05$ & $1.22 \mathrm{E}+20$ & $1.68 \mathrm{E}-05$ & $1.21 \mathrm{E}+20$ & $1.66 \mathrm{E}-05$ & $1.13 \mathrm{E}+20$ & $1.55 \mathrm{E}-05$ \\
\hline $3.60 \mathrm{E}-04$ & 0.01 & $1.31 \mathrm{E}+20$ & $1.64 \mathrm{E}-05$ & $1.25 \mathrm{E}+20$ & 1.56E-05 & $1.18 \mathrm{E}+20$ & 1.47E-05 & $1.12 \mathrm{E}+20$ & $1.40 \mathrm{E}-05$ \\
\hline $4.50 \mathrm{E}-04$ & 0.007 & $1.15 \mathrm{E}+20$ & $1.01 \mathrm{E}-05$ & $1.09 \mathrm{E}+20$ & 9.56E-06 & $1.06 \mathrm{E}+20$ & $9.24 \mathrm{E}-06$ & $9.70 \mathrm{E}+19$ & $8.49 \mathrm{E}-06$ \\
\hline $5.75 \mathrm{E}-04$ & 0.005 & $1.31 \mathrm{E}+20$ & $8.18 \mathrm{E}-06$ & $1.20 \mathrm{E}+20$ & $7.48 \mathrm{E}-06$ & $1.16 \mathrm{E}+20$ & 7.26E-06 & $1.11 \mathrm{E}+20$ & $6.96 \mathrm{E}-06$ \\
\hline 7.60E-04 & 0.129 & $1.49 \mathrm{E}+20$ & $2.41 \mathrm{E}-04$ & $1.38 \mathrm{E}+20$ & 2.23E-04 & $1.33 \mathrm{E}+20$ & 2.15E-04 & $1.29 \mathrm{E}+20$ & $2.08 \mathrm{E}-04$ \\
\hline $9.60 \mathrm{E}-04$ & 0.308 & $1.27 \mathrm{E}+20$ & $4.88 \mathrm{E}-04$ & $1.21 \mathrm{E}+20$ & 4.65E-04 & $1.17 \mathrm{E}+20$ & 4.51E-04 & $1.10 \mathrm{E}+20$ & 4.23E-04 \\
\hline $1.28 \mathrm{E}-03$ & 0.672 & $1.48 \mathrm{E}+20$ & $1.25 \mathrm{E}-03$ & $1.41 \mathrm{E}+20$ & $1.18 \mathrm{E}-03$ & $1.36 \mathrm{E}+20$ & $1.14 \mathrm{E}-03$ & $1.33 \mathrm{E}+20$ & $1.12 \mathrm{E}-03$ \\
\hline $1.60 \mathrm{E}-03$ & 0.477 & $1.19 \mathrm{E}+20$ & $7.12 \mathrm{E}-04$ & $1.14 \mathrm{E}+20$ & $6.80 \mathrm{E}-04$ & $1.05 \mathrm{E}+20$ & $6.26 \mathrm{E}-04$ & $1.02 \mathrm{E}+20$ & $6.09 \mathrm{E}-04$ \\
\hline $2.00 \mathrm{E}-03$ & 0.511 & $1.24 \mathrm{E}+20$ & 7.91E-04 & $1.17 \mathrm{E}+20$ & 7.44E-04 & $1.15 \mathrm{E}+20$ & 7.34E-04 & $1.10 \mathrm{E}+20$ & 7.00E-04 \\
\hline $2.70 \mathrm{E}-03$ & 0.568 & $1.60 \mathrm{E}+20$ & $1.14 \mathrm{E}-03$ & $1.49 \mathrm{E}+20$ & $1.05 \mathrm{E}-03$ & $1.51 \mathrm{E}+20$ & $1.07 \mathrm{E}-03$ & $1.41 \mathrm{E}+20$ & $1.00 \mathrm{E}-03$ \\
\hline $3.40 \mathrm{E}-03$ & 0.632 & $1.27 \mathrm{E}+20$ & $1.01 \mathrm{E}-03$ & $1.20 \mathrm{E}+20$ & $9.46 \mathrm{E}-04$ & 1.17E+20 & $9.22 \mathrm{E}-04$ & $1.13 \mathrm{E}+20$ & $8.91 \mathrm{E}-04$ \\
\hline $4.50 \mathrm{E}-03$ & 0.822 & $1.56 \mathrm{E}+20$ & $1.60 \mathrm{E}-03$ & $1.47 \mathrm{E}+20$ & $1.51 \mathrm{E}-03$ & $1.41 \mathrm{E}+20$ & $1.45 \mathrm{E}-03$ & $1.40 \mathrm{E}+20$ & $1.44 \mathrm{E}-03$ \\
\hline $5.50 \mathrm{E}-03$ & 0.826 & $1.16 \mathrm{E}+20$ & $1.19 \mathrm{E}-03$ & $1.13 \mathrm{E}+20$ & 1.17E-03 & $1.07 \mathrm{E}+20$ & $1.11 \mathrm{E}-03$ & $1.08 \mathrm{E}+20$ & $1.11 \mathrm{E}-03$ \\
\hline $7.20 \mathrm{E}-03$ & 1.671 & $1.51 \mathrm{E}+20$ & 3.15E-03 & $1.42 \mathrm{E}+20$ & 2.97E-03 & $1.43 \mathrm{E}+20$ & 2.99E-03 & $1.39 \mathrm{E}+20$ & 2.90E-03 \\
\hline $9.20 \mathrm{E}-03$ & 3.709 & $1.26 \mathrm{E}+20$ & $5.85 \mathrm{E}-03$ & $1.15 \mathrm{E}+20$ & $5.31 \mathrm{E}-03$ & $1.11 \mathrm{E}+20$ & $5.13 \mathrm{E}-03$ & $1.07 \mathrm{E}+20$ & $4.95 \mathrm{E}-03$ \\
\hline $1.20 \mathrm{E}-02$ & 1.491 & $1.53 \mathrm{E}+20$ & $2.85 \mathrm{E}-03$ & $1.40 \mathrm{E}+20$ & 2.61E-03 & $1.31 \mathrm{E}+20$ & $2.44 \mathrm{E}-03$ & $1.35 \mathrm{E}+20$ & $2.52 \mathrm{E}-03$ \\
\hline $1.50 \mathrm{E}-02$ & 1.156 & $1.31 \mathrm{E}+20$ & $1.89 \mathrm{E}-03$ & $1.23 \mathrm{E}+20$ & $1.78 \mathrm{E}-03$ & $1.16 \mathrm{E}+20$ & $1.68 \mathrm{E}-03$ & $1.14 \mathrm{E}+20$ & $1.65 \mathrm{E}-03$ \\
\hline $1.90 \mathrm{E}-02$ & 0.973 & $1.34 \mathrm{E}+20$ & $1.63 \mathrm{E}-03$ & $1.27 \mathrm{E}+20$ & $1.54 \mathrm{E}-03$ & $1.25 \mathrm{E}+20$ & $1.52 \mathrm{E}-03$ & $1.18 \mathrm{E}+20$ & $1.43 \mathrm{E}-03$ \\
\hline $2.55 \mathrm{E}-02$ & 0.555 & $2.01 \mathrm{E}+20$ & $1.39 \mathrm{E}-03$ & $1.89 \mathrm{E}+20$ & $1.31 \mathrm{E}-03$ & $1.80 \mathrm{E}+20$ & $1.25 \mathrm{E}-03$ & $1.75 \mathrm{E}+20$ & $1.21 \mathrm{E}-03$ \\
\hline $3.20 \mathrm{E}-02$ & 26.762 & $1.78 \mathrm{E}+20$ & 5.96E-02 & $1.65 \mathrm{E}+20$ & $5.53 \mathrm{E}-02$ & $1.60 \mathrm{E}+20$ & $5.35 \mathrm{E}-02$ & $1.56 \mathrm{E}+20$ & $5.24 \mathrm{E}-02$ \\
\hline $4.00 \mathrm{E}-02$ & 8.161 & $8.81 \mathrm{E}+19$ & 8.99E-03 & $8.20 \mathrm{E}+19$ & 8.36E-03 & $7.70 \mathrm{E}+19$ & 7.86E-03 & $7.66 \mathrm{E}+19$ & $7.81 \mathrm{E}-03$ \\
\hline $5.25 \mathrm{E}-02$ & 6.515 & $1.77 \mathrm{E}+20$ & $1.45 \mathrm{E}-02$ & $1.68 \mathrm{E}+20$ & 1.37E-02 & $1.57 \mathrm{E}+20$ & $1.28 \mathrm{E}-02$ & $1.55 \mathrm{E}+20$ & $1.26 \mathrm{E}-02$ \\
\hline $6.60 \mathrm{E}-02$ & 6.576 & $1.77 \mathrm{E}+20$ & $1.45 \mathrm{E}-02$ & $1.63 \mathrm{E}+20$ & $1.34 \mathrm{E}-02$ & $1.60 \mathrm{E}+20$ & $1.31 \mathrm{E}-02$ & $1.50 \mathrm{E}+20$ & $1.23 \mathrm{E}-02$ \\
\hline $8.80 \mathrm{E}-02$ & 13.137 & $2.63 \mathrm{E}+20$ & 4.31E-02 & $2.44 \mathrm{E}+20$ & 4.01E-02 & $2.31 \mathrm{E}+20$ & $3.79 \mathrm{E}-02$ & $2.23 \mathrm{E}+20$ & 3.67E-02 \\
\hline $1.10 \mathrm{E}-01$ & 11.84 & $1.59 \mathrm{E}+20$ & $2.35 \mathrm{E}-02$ & $1.46 \mathrm{E}+20$ & 2.17E-02 & $1.40 \mathrm{E}+20$ & 2.07E-02 & $1.39 \mathrm{E}+20$ & $2.06 \mathrm{E}-02$ \\
\hline $1.35 \mathrm{E}-01$ & 11.27 & $2.34 \mathrm{E}+20$ & $3.30 \mathrm{E}-02$ & $2.16 \mathrm{E}+20$ & $3.04 \mathrm{E}-02$ & $2.07 \mathrm{E}+20$ & $2.92 \mathrm{E}-02$ & $2.04 \mathrm{E}+20$ & $2.87 \mathrm{E}-02$ \\
\hline $1.60 \mathrm{E}-01$ & 21.53 & $1.40 \mathrm{E}+20$ & 3.77E-02 & $1.29 \mathrm{E}+20$ & $3.46 \mathrm{E}-02$ & $1.22 \mathrm{E}+20$ & $3.29 \mathrm{E}-02$ & $1.23 \mathrm{E}+20$ & $3.31 \mathrm{E}-02$ \\
\hline $1.90 \mathrm{E}-01$ & 15.85 & $1.92 \mathrm{E}+20$ & $3.80 \mathrm{E}-02$ & $1.77 \mathrm{E}+20$ & $3.50 \mathrm{E}-02$ & $1.74 \mathrm{E}+20$ & 3.44E-02 & $1.65 \mathrm{E}+20$ & $3.26 \mathrm{E}-02$ \\
\hline $2.20 \mathrm{E}-01$ & 22.01 & $1.56 \mathrm{E}+20$ & 4.30E-02 & $1.43 \mathrm{E}+20$ & 3.94E-02 & $1.37 \mathrm{E}+20$ & 3.77E-02 & $1.35 \mathrm{E}+20$ & $3.70 \mathrm{E}-02$ \\
\hline
\end{tabular}




\begin{tabular}{|c|c|c|c|c|c|c|c|c|c|}
\hline \multirow[b]{2}{*}{$\begin{array}{c}\text { Upper } \\
\text { Energy(MeV) } \\
\end{array}$} & \multirow{2}{*}{$\begin{array}{l}\text { DPA xsec } \\
\text { keV-barn }\end{array}$} & \multicolumn{2}{|c|}{$3-4$} & \multicolumn{2}{|c|}{ 3-3 } & \multicolumn{2}{|c|}{$3-2$} & \multicolumn{2}{|c|}{ 3-1 } \\
\hline & & Total Fluence & DPA & Total Fluence & DPA & Total Fluence & DPA & Total Fluence & DPA \\
\hline $2.55 \mathrm{E}-01$ & 18.27 & $1.80 \mathrm{E}+20$ & 4.12E-02 & $1.70 \mathrm{E}+20$ & $3.88 \mathrm{E}-02$ & $1.64 \mathrm{E}+20$ & $3.74 \mathrm{E}-02$ & $1.56 \mathrm{E}+20$ & $3.56 \mathrm{E}-02$ \\
\hline $2.90 \mathrm{E}-01$ & 17.37 & $1.94 \mathrm{E}+20$ & 4.20E-02 & $1.86 \mathrm{E}+20$ & 4.03E-02 & $1.80 \mathrm{E}+20$ & 3.90E-02 & $1.76 \mathrm{E}+20$ & $3.81 \mathrm{E}-02$ \\
\hline $3.20 \mathrm{E}-01$ & 13.51 & $1.39 \mathrm{E}+20$ & $2.34 \mathrm{E}-02$ & $1.27 \mathrm{E}+20$ & $2.15 \mathrm{E}-02$ & $1.22 \mathrm{E}+20$ & $2.06 \mathrm{E}-02$ & $1.22 \mathrm{E}+20$ & 2.06E-02 \\
\hline $3.60 \mathrm{E}-01$ & 25.14 & $1.93 \mathrm{E}+20$ & $6.06 \mathrm{E}-02$ & $1.80 \mathrm{E}+20$ & 5.65E-02 & $1.78 \mathrm{E}+20$ & $5.59 \mathrm{E}-02$ & $1.68 \mathrm{E}+20$ & 5.27E-02 \\
\hline 4.00E-01 & 46.52 & $1.69 \mathrm{E}+20$ & $9.82 \mathrm{E}-02$ & $1.57 \mathrm{E}+20$ & $9.11 \mathrm{E}-02$ & $1.52 \mathrm{E}+20$ & $8.85 \mathrm{E}-02$ & $1.48 \mathrm{E}+20$ & $8.59 \mathrm{E}-02$ \\
\hline $4.50 \mathrm{E}-01$ & 43.04 & $1.58 \mathrm{E}+20$ & $8.48 \mathrm{E}-02$ & $1.43 \mathrm{E}+20$ & 7.69E-02 & $1.41 \mathrm{E}+20$ & 7.61E-02 & $1.41 \mathrm{E}+20$ & $7.58 \mathrm{E}-02$ \\
\hline $5.00 \mathrm{E}-01$ & 36.4 & $1.78 \mathrm{E}+20$ & 8.11E-02 & $1.67 \mathrm{E}+20$ & 7.59E-02 & $1.62 \mathrm{E}+20$ & 7.36E-02 & $1.60 \mathrm{E}+20$ & 7.26E-02 \\
\hline $5.50 \mathrm{E}-01$ & 33.22 & $1.77 \mathrm{E}+20$ & 7.33E-02 & $1.70 \mathrm{E}+20$ & 7.06E-02 & $1.59 \mathrm{E}+20$ & $6.62 \mathrm{E}-02$ & $1.58 \mathrm{E}+20$ & $6.58 \mathrm{E}-02$ \\
\hline $6.00 \mathrm{E}-01$ & 34.18 & $1.69 \mathrm{E}+20$ & 7.23E-02 & $1.63 \mathrm{E}+20$ & 6.97E-02 & $1.55 \mathrm{E}+20$ & 6.63E-02 & $1.43 \mathrm{E}+20$ & $6.10 \mathrm{E}-02$ \\
\hline $6.60 \mathrm{E}-01$ & 19.64 & $2.07 \mathrm{E}+20$ & 5.09E-02 & $1.98 \mathrm{E}+20$ & 4.86E-02 & $1.82 \mathrm{E}+20$ & $4.48 \mathrm{E}-02$ & $1.87 \mathrm{E}+20$ & $4.58 \mathrm{E}-02$ \\
\hline $7.20 \mathrm{E}-01$ & 48.89 & $2.15 \mathrm{E}+20$ & $1.31 \mathrm{E}-01$ & $1.96 \mathrm{E}+20$ & $1.20 \mathrm{E}-01$ & $1.96 \mathrm{E}+20$ & $1.20 \mathrm{E}-01$ & $1.93 \mathrm{E}+20$ & $1.18 \mathrm{E}-01$ \\
\hline $7.80 \mathrm{E}-01$ & 74.22 & $2.05 E+20$ & $1.90 \mathrm{E}-01$ & $1.87 \mathrm{E}+20$ & $1.73 \mathrm{E}-01$ & $1.83 \mathrm{E}+20$ & $1.70 \mathrm{E}-01$ & $1.79 \mathrm{E}+20$ & $1.66 \mathrm{E}-01$ \\
\hline $8.40 \mathrm{E}-01$ & 44.02 & $1.58 \mathrm{E}+20$ & $8.72 \mathrm{E}-02$ & $1.51 \mathrm{E}+20$ & $8.31 \mathrm{E}-02$ & $1.44 \mathrm{E}+20$ & 7.93E-02 & $1.40 \mathrm{E}+20$ & $7.69 \mathrm{E}-02$ \\
\hline $9.20 \mathrm{E}-01$ & 40.97 & $1.91 \mathrm{E}+20$ & $9.80 \mathrm{E}-02$ & $1.79 \mathrm{E}+20$ & $9.18 \mathrm{E}-02$ & $1.72 \mathrm{E}+20$ & 8.83E-02 & $1.68 \mathrm{E}+20$ & $8.59 \mathrm{E}-02$ \\
\hline $1.00 \mathrm{E}+00$ & 50.95 & $1.75 \mathrm{E}+20$ & $1.12 \mathrm{E}-01$ & $1.64 \mathrm{E}+20$ & $1.05 \mathrm{E}-01$ & $1.58 \mathrm{E}+20$ & $1.00 \mathrm{E}-01$ & $1.54 \mathrm{E}+20$ & $9.80 \mathrm{E}-02$ \\
\hline $1.20 \mathrm{E}+00$ & 50.09 & $4.16 \mathrm{E}+20$ & $2.61 \mathrm{E}-01$ & $3.91 \mathrm{E}+20$ & $2.45 \mathrm{E}-01$ & $3.67 \mathrm{E}+20$ & $2.30 \mathrm{E}-01$ & $3.60 \mathrm{E}+20$ & $2.25 \mathrm{E}-01$ \\
\hline $1.40 \mathrm{E}+00$ & 64.54 & $3.67 \mathrm{E}+20$ & 2.96E-01 & $3.43 \mathrm{E}+20$ & 2.77E-01 & $3.30 \mathrm{E}+20$ & $2.66 \mathrm{E}-01$ & $3.19 \mathrm{E}+20$ & 2.57E-01 \\
\hline $1.60 \mathrm{E}+00$ & 73.58 & $3.15 \mathrm{E}+20$ & 2.90E-01 & $2.90 \mathrm{E}+20$ & 2.67E-01 & $2.92 \mathrm{E}+20$ & $2.69 \mathrm{E}-01$ & $2.70 \mathrm{E}+20$ & $2.48 \mathrm{E}-01$ \\
\hline $1.80 \mathrm{E}+00$ & 76.46 & $2.78 \mathrm{E}+20$ & $2.66 \mathrm{E}-01$ & $2.56 \mathrm{E}+20$ & $2.44 \mathrm{E}-01$ & $2.47 \mathrm{E}+20$ & $2.36 \mathrm{E}-01$ & $2.38 \mathrm{E}+20$ & $2.28 \mathrm{E}-01$ \\
\hline $2.00 \mathrm{E}+00$ & 95.15 & $2.45 \mathrm{E}+20$ & $2.91 \mathrm{E}-01$ & $2.23 \mathrm{E}+20$ & $2.66 \mathrm{E}-01$ & $2.22 \mathrm{E}+20$ & $2.64 \mathrm{E}-01$ & $2.09 \mathrm{E}+20$ & $2.48 \mathrm{E}-01$ \\
\hline $2.30 \mathrm{E}+00$ & 93.75 & $3.04 \mathrm{E}+20$ & 3.56E-01 & $2.81 \mathrm{E}+20$ & 3.29E-01 & $2.72 \mathrm{E}+20$ & 3.18E-01 & $2.57 \mathrm{E}+20$ & $3.02 \mathrm{E}-01$ \\
\hline $2.60 \mathrm{E}+00$ & 112.05 & $2.79 \mathrm{E}+20$ & $3.91 \mathrm{E}-01$ & $2.56 \mathrm{E}+20$ & $3.59 \mathrm{E}-01$ & $2.46 \mathrm{E}+20$ & $3.44 \mathrm{E}-01$ & $2.37 \mathrm{E}+20$ & 3.33E-01 \\
\hline $2.90 \mathrm{E}+00$ & 123.55 & $2.09 \mathrm{E}+20$ & $3.23 \mathrm{E}-01$ & $1.99 \mathrm{E}+20$ & 3.07E-01 & $1.84 \mathrm{E}+20$ & $2.84 \mathrm{E}-01$ & $1.83 \mathrm{E}+20$ & 2.83E-01 \\
\hline $3.30 \mathrm{E}+00$ & 133.45 & $2.13 E+20$ & 3.56E-01 & $1.91 \mathrm{E}+20$ & 3.19E-01 & $1.88 \mathrm{E}+20$ & $3.14 \mathrm{E}-01$ & $1.81 \mathrm{E}+20$ & 3.03E-01 \\
\hline $3.70 \mathrm{E}+00$ & 135.25 & $1.42 \mathrm{E}+20$ & 2.39E-01 & $1.34 \mathrm{E}+20$ & 2.27E-01 & $1.28 \mathrm{E}+20$ & 2.17E-01 & $1.22 \mathrm{E}+20$ & 2.06E-01 \\
\hline $4.10 \mathrm{E}+00$ & 149.55 & $1.11 \mathrm{E}+20$ & $2.08 \mathrm{E}-01$ & $1.04 \mathrm{E}+20$ & $1.95 \mathrm{E}-01$ & $9.70 \mathrm{E}+19$ & $1.81 \mathrm{E}-01$ & $9.45 \mathrm{E}+19$ & 1.77E-01 \\
\hline $4.50 \mathrm{E}+00$ & 158.25 & $8.63 E+19$ & $1.71 \mathrm{E}-01$ & $7.88 \mathrm{E}+19$ & $1.56 \mathrm{E}-01$ & $7.90 \mathrm{E}+19$ & $1.56 \mathrm{E}-01$ & $7.66 \mathrm{E}+19$ & $1.52 \mathrm{E}-01$ \\
\hline $5.00 \mathrm{E}+00$ & 168.55 & $7.97 \mathrm{E}+19$ & $1.68 \mathrm{E}-01$ & $7.61 \mathrm{E}+19$ & $1.60 \mathrm{E}-01$ & $7.18 \mathrm{E}+19$ & $1.51 \mathrm{E}-01$ & $7.00 \mathrm{E}+19$ & 1.47E-01 \\
\hline $5.50 \mathrm{E}+00$ & 176.46 & $5.39 \mathrm{E}+19$ & 1.19E-01 & $5.18 \mathrm{E}+19$ & 1.14E-01 & $5.06 \mathrm{E}+19$ & $1.12 \mathrm{E}-01$ & 4.95E+19 & $1.09 \mathrm{E}-01$ \\
\hline
\end{tabular}




\begin{tabular}{|c|c|c|c|c|c|c|c|c|c|}
\hline \multirow{2}{*}{$\begin{array}{c}\text { Upper } \\
\text { Energy(MeV) }\end{array}$} & \multirow{2}{*}{$\begin{array}{l}\text { DPA xsec } \\
\text { keV-barn }\end{array}$} & \multicolumn{2}{|c|}{$3-4$} & \multicolumn{2}{|c|}{ 3-3 } & \multicolumn{2}{|c|}{$3-2$} & \multicolumn{2}{|c|}{ 3-1 } \\
\hline & & Total Fluence & DPA & Total Fluence & DPA & Total Fluence & DPA & Total Fluence & DPA \\
\hline $6.00 \mathrm{E}+00$ & 183.06 & $3.91 \mathrm{E}+19$ & $8.94 \mathrm{E}-02$ & $3.32 E+19$ & 7.60E-02 & $3.23 E+19$ & 7.40E-02 & $3.17 E+19$ & $7.25 \mathrm{E}-02$ \\
\hline $6.70 \mathrm{E}+00$ & 189.26 & $3.55 E+19$ & $8.39 \mathrm{E}-02$ & $3.33 \mathrm{E}+19$ & $7.88 \mathrm{E}-02$ & $3.29 E+19$ & 7.78E-02 & $3.14 \mathrm{E}+19$ & $7.43 \mathrm{E}-02$ \\
\hline $7.40 \mathrm{E}+00$ & 196.67 & $1.98 \mathrm{E}+19$ & 4.87E-02 & $2.02 \mathrm{E}+19$ & 4.96E-02 & $1.95 \mathrm{E}+19$ & 4.80E-02 & $1.97 \mathrm{E}+19$ & 4.84E-02 \\
\hline $8.20 \mathrm{E}+00$ & 203.37 & $1.28 \mathrm{E}+19$ & $3.24 \mathrm{E}-02$ & $1.24 \mathrm{E}+19$ & $3.15 \mathrm{E}-02$ & $1.19 \mathrm{E}+19$ & $3.03 \mathrm{E}-02$ & $1.12 \mathrm{E}+19$ & $2.86 \mathrm{E}-02$ \\
\hline $9.00 \mathrm{E}+00$ & 214.58 & $7.30 \mathrm{E}+18$ & $1.96 \mathrm{E}-02$ & $7.04 \mathrm{E}+18$ & $1.89 \mathrm{E}-02$ & $5.76 \mathrm{E}+18$ & $1.55 \mathrm{E}-02$ & $5.50 \mathrm{E}+18$ & $1.48 \mathrm{E}-02$ \\
\hline $1.00 \mathrm{E}+01$ & 225.69 & $4.19 E+18$ & $1.18 \mathrm{E}-02$ & $4.39 E+18$ & $1.24 \mathrm{E}-02$ & $3.50 E+18$ & $9.87 \mathrm{E}-03$ & $3.38 \mathrm{E}+18$ & $9.54 \mathrm{E}-03$ \\
\hline $1.10 \mathrm{E}+01$ & 237.6 & $2.09 E+18$ & $6.21 \mathrm{E}-03$ & $2.40 \mathrm{E}+18$ & $7.12 \mathrm{E}-03$ & $1.74 \mathrm{E}+18$ & $5.16 \mathrm{E}-03$ & $1.62 \mathrm{E}+18$ & 4.81E-03 \\
\hline $1.20 \mathrm{E}+01$ & 247.41 & $7.25 \mathrm{E}+17$ & $2.24 \mathrm{E}-03$ & $1.02 \mathrm{E}+18$ & 3.17E-03 & $8.72 E+17$ & 2.70E-03 & $5.91 \mathrm{E}+17$ & $1.83 \mathrm{E}-03$ \\
\hline $1.30 \mathrm{E}+01$ & 258.52 & $4.29 \mathrm{E}+17$ & $1.39 \mathrm{E}-03$ & $2.76 \mathrm{E}+17$ & 8.93E-04 & $1.84 \mathrm{E}+17$ & $5.94 \mathrm{E}-04$ & $6.08 \mathrm{E}+17$ & $1.96 \mathrm{E}-03$ \\
\hline $1.40 \mathrm{E}+01$ & 271.35 & $1.33 \mathrm{E}+17$ & 4.51E-04 & $1.82 \mathrm{E}+17$ & $6.16 \mathrm{E}-04$ & $1.36 \mathrm{E}+17$ & 4.61E-04 & $4.08 \mathrm{E}+16$ & 1.39E-04 \\
\hline $1.50 \mathrm{E}+01$ & 290.27 & $3.34 \mathrm{E}+16$ & $1.21 \mathrm{E}-04$ & $7.55 E+16$ & $2.74 \mathrm{E}-04$ & $5.94 \mathrm{E}+16$ & $2.16 \mathrm{E}-04$ & $0.00 \mathrm{E}+00$ & $0.00 \mathrm{E}+00$ \\
\hline $1.60 \mathrm{E}+01$ & 293.2 & $1.67 \mathrm{E}+16$ & $6.11 \mathrm{E}-05$ & $5.16 \mathrm{E}+16$ & $1.89 \mathrm{E}-04$ & $0.00 \mathrm{E}+00$ & $0.00 \mathrm{E}+00$ & $0.00 \mathrm{E}+00$ & $0.00 \mathrm{E}+00$ \\
\hline $1.70 \mathrm{E}+01$ & 292.73 & $0.00 \mathrm{E}+00$ & $0.00 \mathrm{E}+00$ & $0.00 \mathrm{E}+00$ & $0.00 \mathrm{E}+00$ & $5.82 \mathrm{E}+16$ & $2.13 \mathrm{E}-04$ & $0.00 \mathrm{E}+00$ & $0.00 \mathrm{E}+00$ \\
\hline $1.80 \mathrm{E}+01$ & 297.65 & $1.11 \mathrm{E}+16$ & $4.12 \mathrm{E}-05$ & $0.00 \mathrm{E}+00$ & $0.00 \mathrm{E}+00$ & $0.00 \mathrm{E}+00$ & $0.00 \mathrm{E}+00$ & $0.00 \mathrm{E}+00$ & $0.00 \mathrm{E}+00$ \\
\hline $1.90 \mathrm{E}+01$ & 307.26 & $0.00 \mathrm{E}+00$ & $0.00 \mathrm{E}+00$ & $0.00 \mathrm{E}+00$ & $0.00 \mathrm{E}+00$ & $0.00 \mathrm{E}+00$ & $0.00 \mathrm{E}+00$ & $1.07 \mathrm{E}+17$ & 4.12E-04 \\
\hline \multirow[t]{2}{*}{$2.00 \mathrm{E}+01$} & 316.36 & $0.00 \mathrm{E}+00$ & $0.00 \mathrm{E}+00$ & $0.00 \mathrm{E}+00$ & $0.00 \mathrm{E}+00$ & $0.00 \mathrm{E}+00$ & $0.00 \mathrm{E}+00$ & $0.00 \mathrm{E}+00$ & $0.00 \mathrm{E}+00$ \\
\hline & & & $5.65 E+00$ & & $5.25 \mathrm{E}+00$ & & $5.07 \mathrm{E}+00$ & & $4.90 \mathrm{E}+00$ \\
\hline
\end{tabular}


Table 19. Calculated fluence and DPA for capsule 2 of the UCSB experiment.

\begin{tabular}{|c|c|c|c|c|c|c|c|c|c|}
\hline \multirow{2}{*}{$\begin{array}{c}\text { Upper } \\
\text { Energy(MeV) }\end{array}$} & \multirow{2}{*}{$\begin{array}{l}\text { DPA xsec } \\
\text { keV-barn }\end{array}$} & \multicolumn{2}{|c|}{$2-7$} & \multicolumn{2}{|c|}{$2-6$} & \multicolumn{2}{|c|}{$2-5$} & \multicolumn{2}{|c|}{$2-4$} \\
\hline & & Total Fluence & DPA & Total Fluence & DPA & Total Fluence & DPA & Total Fluence & DPA \\
\hline $1.00 \mathrm{E}-10$ & 0 & $0.00 \mathrm{E}+00$ & & $8.74 \mathrm{E}+13$ & & $6.52 \mathrm{E}+15$ & & $0.00 \mathrm{E}+00$ & \\
\hline $1.00 \mathrm{E}-09$ & 7.73 & $5.22 \mathrm{E}+17$ & $5.04 \mathrm{E}-05$ & $3.98 \mathrm{E}+17$ & $3.85 \mathrm{E}-05$ & $4.41 \mathrm{E}+17$ & $4.26 \mathrm{E}-05$ & $4.72 \mathrm{E}+17$ & 4.56E-05 \\
\hline $1.00 \mathrm{E}-08$ & 2.444 & $7.85 E+19$ & $2.40 \mathrm{E}-03$ & $6.66 \mathrm{E}+19$ & $2.03 \mathrm{E}-03$ & $6.47 \mathrm{E}+19$ & $1.98 \mathrm{E}-03$ & $6.20 \mathrm{E}+19$ & $1.89 \mathrm{E}-03$ \\
\hline $2.30 \mathrm{E}-08$ & 1.278 & $2.75 E+20$ & $4.40 \mathrm{E}-03$ & $2.49 \mathrm{E}+20$ & $3.98 \mathrm{E}-03$ & $2.46 \mathrm{E}+20$ & $3.93 E-03$ & $2.28 \mathrm{E}+20$ & $3.64 \mathrm{E}-03$ \\
\hline $5.00 \mathrm{E}-08$ & 0.857 & $6.83 E+20$ & $7.32 \mathrm{E}-03$ & $6.14 \mathrm{E}+20$ & $6.58 \mathrm{E}-03$ & $6.07 E+20$ & $6.50 \mathrm{E}-03$ & $5.67 E+20$ & $6.08 \mathrm{E}-03$ \\
\hline 7.60E-08 & 0.644 & $4.91 E+20$ & $3.95 \mathrm{E}-03$ & $4.55 E+20$ & $3.67 \mathrm{E}-03$ & $4.41 \mathrm{E}+20$ & $3.55 \mathrm{E}-03$ & $4.12 \mathrm{E}+20$ & $3.32 \mathrm{E}-03$ \\
\hline $1.15 \mathrm{E}-07$ & 0.523 & $4.27 \mathrm{E}+20$ & $2.79 \mathrm{E}-03$ & $3.93 \mathrm{E}+20$ & $2.57 \mathrm{E}-03$ & $3.70 \mathrm{E}+20$ & $2.42 \mathrm{E}-03$ & $3.52 \mathrm{E}+20$ & $2.30 \mathrm{E}-03$ \\
\hline $1.70 \mathrm{E}-07$ & 0.428 & $2.69 \mathrm{E}+20$ & $1.44 \mathrm{E}-03$ & $2.54 \mathrm{E}+20$ & $1.36 \mathrm{E}-03$ & $2.42 \mathrm{E}+20$ & $1.29 \mathrm{E}-03$ & $2.35 \mathrm{E}+20$ & $1.25 \mathrm{E}-03$ \\
\hline $2.55 \mathrm{E}-07$ & 0.351 & $1.91 \mathrm{E}+20$ & $8.40 \mathrm{E}-04$ & $1.82 \mathrm{E}+20$ & 8.00E-04 & $1.79 \mathrm{E}+20$ & $7.85 \mathrm{E}-04$ & $1.66 \mathrm{E}+20$ & 7.29E-04 \\
\hline $3.80 \mathrm{E}-07$ & 0.287 & $1.65 E+20$ & 5.90E-04 & $1.55 \mathrm{E}+20$ & $5.55 \mathrm{E}-04$ & $1.47 \mathrm{E}+20$ & $5.29 \mathrm{E}-04$ & $1.42 \mathrm{E}+20$ & 5.09E-04 \\
\hline $5.50 \mathrm{E}-07$ & 0.237 & $1.50 \mathrm{E}+20$ & $4.45 \mathrm{E}-04$ & $1.44 \mathrm{E}+20$ & 4.27E-04 & $1.34 \mathrm{E}+20$ & 3.96E-04 & $1.24 \mathrm{E}+20$ & $3.69 \mathrm{E}-04$ \\
\hline $8.40 \mathrm{E}-07$ & 0.194 & $1.70 \mathrm{E}+20$ & 4.13E-04 & $1.55 \mathrm{E}+20$ & $3.75 \mathrm{E}-04$ & $1.46 \mathrm{E}+20$ & $3.54 \mathrm{E}-04$ & $1.35 \mathrm{E}+20$ & 3.27E-04 \\
\hline $1.28 \mathrm{E}-06$ & 0.157 & $1.63 \mathrm{E}+20$ & $3.20 \mathrm{E}-04$ & $1.55 \mathrm{E}+20$ & $3.04 \mathrm{E}-04$ & $1.50 \mathrm{E}+20$ & $2.95 \mathrm{E}-04$ & $1.36 \mathrm{E}+20$ & 2.67E-04 \\
\hline $1.90 \mathrm{E}-06$ & 0.128 & $1.56 \mathrm{E}+20$ & $2.50 \mathrm{E}-04$ & $1.46 \mathrm{E}+20$ & $2.34 \mathrm{E}-04$ & $1.37 \mathrm{E}+20$ & $2.19 \mathrm{E}-04$ & $1.31 \mathrm{E}+20$ & $2.10 \mathrm{E}-04$ \\
\hline $2.80 \mathrm{E}-06$ & 0.105 & $1.51 \mathrm{E}+20$ & $1.98 \mathrm{E}-04$ & $1.36 \mathrm{E}+20$ & $1.79 \mathrm{E}-04$ & $1.34 \mathrm{E}+20$ & $1.76 \mathrm{E}-04$ & $1.24 \mathrm{E}+20$ & $1.62 \mathrm{E}-04$ \\
\hline $4.25 \mathrm{E}-06$ & 0.086 & $1.57 \mathrm{E}+20$ & $1.69 \mathrm{E}-04$ & $1.27 \mathrm{E}+20$ & $1.36 \mathrm{E}-04$ & $1.34 \mathrm{E}+20$ & $1.44 \mathrm{E}-04$ & $1.32 \mathrm{E}+20$ & $1.42 \mathrm{E}-04$ \\
\hline $6.30 \mathrm{E}-06$ & 0.07 & $1.46 \mathrm{E}+20$ & $1.27 \mathrm{E}-04$ & $1.33 \mathrm{E}+20$ & $1.16 \mathrm{E}-04$ & $1.29 \mathrm{E}+20$ & $1.13 \mathrm{E}-04$ & $1.24 \mathrm{E}+20$ & $1.08 \mathrm{E}-04$ \\
\hline $9.20 \mathrm{E}-06$ & 0.058 & $1.39 \mathrm{E}+20$ & $1.01 \mathrm{E}-04$ & $1.21 \mathrm{E}+20$ & $8.75 \mathrm{E}-05$ & $1.26 \mathrm{E}+20$ & $9.12 \mathrm{E}-05$ & $1.20 \mathrm{E}+20$ & 8.73E-05 \\
\hline $1.35 \mathrm{E}-05$ & 0.048 & $1.54 \mathrm{E}+20$ & $9.23 \mathrm{E}-05$ & $1.36 \mathrm{E}+20$ & 8.19E-05 & $1.27 \mathrm{E}+20$ & 7.64E-05 & $1.28 \mathrm{E}+20$ & 7.67E-05 \\
\hline $2.10 \mathrm{E}-05$ & 0.038 & $1.68 \mathrm{E}+20$ & $7.96 \mathrm{E}-05$ & $1.19 \mathrm{E}+20$ & $5.63 \mathrm{E}-05$ & $1.44 \mathrm{E}+20$ & $6.83 \mathrm{E}-05$ & $1.42 \mathrm{E}+20$ & 6.76E-05 \\
\hline $3.00 \mathrm{E}-05$ & 0.031 & $1.40 \mathrm{E}+20$ & $5.41 \mathrm{E}-05$ & $1.14 \mathrm{E}+20$ & 4.43E-05 & $1.27 \mathrm{E}+20$ & 4.93E-05 & $1.19 \mathrm{E}+20$ & 4.63E-05 \\
\hline $4.50 \mathrm{E}-05$ & 0.026 & $1.66 \mathrm{E}+20$ & $5.40 \mathrm{E}-05$ & $1.50 \mathrm{E}+20$ & 4.87E-05 & $1.47 \mathrm{E}+20$ & 4.78E-05 & $1.33 \mathrm{E}+20$ & 4.32E-05 \\
\hline $6.90 \mathrm{E}-05$ & 0.021 & $1.82 \mathrm{E}+20$ & 4.77E-05 & $1.59 \mathrm{E}+20$ & 4.17E-05 & $1.56 \mathrm{E}+20$ & 4.10E-05 & $1.45 \mathrm{E}+20$ & $3.79 \mathrm{E}-05$ \\
\hline $1.00 \mathrm{E}-04$ & 0.017 & $1.51 \mathrm{E}+20$ & $3.22 \mathrm{E}-05$ & $1.46 \mathrm{E}+20$ & $3.10 \mathrm{E}-05$ & $1.39 \mathrm{E}+20$ & $2.95 \mathrm{E}-05$ & $1.29 \mathrm{E}+20$ & $2.75 \mathrm{E}-05$ \\
\hline $1.35 \mathrm{E}-04$ & 0.014 & $1.21 \mathrm{E}+20$ & $2.12 \mathrm{E}-05$ & $1.12 \mathrm{E}+20$ & $1.96 \mathrm{E}-05$ & $1.07 \mathrm{E}+20$ & $1.86 \mathrm{E}-05$ & $1.05 E+20$ & $1.83 \mathrm{E}-05$ \\
\hline $1.70 \mathrm{E}-04$ & 0.014 & $9.47 \mathrm{E}+19$ & $1.66 \mathrm{E}-05$ & $9.03 E+19$ & $1.58 \mathrm{E}-05$ & $8.79 E+19$ & $1.54 \mathrm{E}-05$ & $8.41 \mathrm{E}+19$ & 1.47E-05 \\
\hline $2.20 \mathrm{E}-04$ & 0.011 & $1.07 \mathrm{E}+20$ & $1.47 \mathrm{E}-05$ & $9.92 E+19$ & $1.36 \mathrm{E}-05$ & $9.75 E+19$ & $1.34 \mathrm{E}-05$ & $9.36 \mathrm{E}+19$ & $1.29 \mathrm{E}-05$ \\
\hline
\end{tabular}




\begin{tabular}{|c|c|c|c|c|c|c|c|c|c|}
\hline \multirow[b]{2}{*}{$\begin{array}{c}\text { Upper } \\
\text { Energy(MeV) }\end{array}$} & \multirow{2}{*}{$\begin{array}{l}\text { DPA xsec } \\
\text { keV-barn }\end{array}$} & \multicolumn{2}{|c|}{$2-7$} & \multicolumn{2}{|c|}{$2-6$} & \multicolumn{2}{|c|}{$2-5$} & \multicolumn{2}{|c|}{$2-4$} \\
\hline & & Total Fluence & DPA & Total Fluence & DPA & Total Fluence & DPA & Total Fluence & DPA \\
\hline $2.80 \mathrm{E}-04$ & 0.011 & $1.07 \mathrm{E}+20$ & 1.47E-05 & $9.32 \mathrm{E}+19$ & $1.28 \mathrm{E}-05$ & $9.27 E+19$ & 1.27E-05 & $8.99 \mathrm{E}+19$ & $1.24 \mathrm{E}-05$ \\
\hline $3.60 \mathrm{E}-04$ & 0.01 & $1.04 \mathrm{E}+20$ & $1.30 \mathrm{E}-05$ & $9.85 \mathrm{E}+19$ & $1.23 \mathrm{E}-05$ & $9.32 \mathrm{E}+19$ & 1.17E-05 & $8.67 \mathrm{E}+19$ & $1.08 \mathrm{E}-05$ \\
\hline $4.50 \mathrm{E}-04$ & 0.007 & $9.20 \mathrm{E}+19$ & 8.05E-06 & $8.66 \mathrm{E}+19$ & 7.57E-06 & $8.40 \mathrm{E}+19$ & 7.35E-06 & $7.09 \mathrm{E}+19$ & $6.21 \mathrm{E}-06$ \\
\hline $5.75 \mathrm{E}-04$ & 0.005 & $1.05 \mathrm{E}+20$ & $6.55 \mathrm{E}-06$ & $9.47 \mathrm{E}+19$ & $5.92 \mathrm{E}-06$ & $8.97 \mathrm{E}+19$ & $5.60 \mathrm{E}-06$ & $8.84 \mathrm{E}+19$ & $5.52 \mathrm{E}-06$ \\
\hline $7.60 \mathrm{E}-04$ & 0.129 & $1.22 \mathrm{E}+20$ & 1.97E-04 & $1.11 \mathrm{E}+20$ & $1.79 \mathrm{E}-04$ & $1.06 \mathrm{E}+20$ & $1.70 \mathrm{E}-04$ & $1.00 \mathrm{E}+20$ & $1.61 \mathrm{E}-04$ \\
\hline $9.60 \mathrm{E}-04$ & 0.308 & $1.02 \mathrm{E}+20$ & 3.91E-04 & $9.40 \mathrm{E}+19$ & $3.62 \mathrm{E}-04$ & $9.07 E+19$ & $3.49 \mathrm{E}-04$ & $8.18 \mathrm{E}+19$ & 3.15E-04 \\
\hline $1.28 \mathrm{E}-03$ & 0.672 & $1.17 \mathrm{E}+20$ & $9.81 \mathrm{E}-04$ & $1.09 \mathrm{E}+20$ & 9.12E-04 & $1.07 \mathrm{E}+20$ & $8.98 \mathrm{E}-04$ & $1.02 \mathrm{E}+20$ & $8.59 \mathrm{E}-04$ \\
\hline $1.60 \mathrm{E}-03$ & 0.477 & $9.82 \mathrm{E}+19$ & $5.86 \mathrm{E}-04$ & $8.99 \mathrm{E}+19$ & $5.36 \mathrm{E}-04$ & $8.50 \mathrm{E}+19$ & 5.07E-04 & $8.43 E+19$ & $5.03 \mathrm{E}-04$ \\
\hline $2.00 \mathrm{E}-03$ & 0.511 & $1.01 \mathrm{E}+20$ & $6.45 \mathrm{E}-04$ & $9.05 E+19$ & $5.78 \mathrm{E}-04$ & $8.81 \mathrm{E}+19$ & $5.63 \mathrm{E}-04$ & $7.95 \mathrm{E}+19$ & $5.08 \mathrm{E}-04$ \\
\hline $2.70 \mathrm{E}-03$ & 0.568 & $1.33 \mathrm{E}+20$ & $9.43 \mathrm{E}-04$ & $1.24 \mathrm{E}+20$ & $8.80 \mathrm{E}-04$ & $1.12 \mathrm{E}+20$ & $7.92 \mathrm{E}-04$ & $1.08 \mathrm{E}+20$ & $7.65 \mathrm{E}-04$ \\
\hline $3.40 \mathrm{E}-03$ & 0.632 & $1.02 \mathrm{E}+20$ & 8.07E-04 & $9.20 \mathrm{E}+19$ & 7.26E-04 & $9.04 \mathrm{E}+19$ & 7.14E-04 & $8.82 \mathrm{E}+19$ & 6.97E-04 \\
\hline $4.50 \mathrm{E}-03$ & 0.822 & $1.23 \mathrm{E}+20$ & $1.26 \mathrm{E}-03$ & $1.16 \mathrm{E}+20$ & $1.19 \mathrm{E}-03$ & $1.11 \mathrm{E}+20$ & $1.14 \mathrm{E}-03$ & $1.09 \mathrm{E}+20$ & $1.11 \mathrm{E}-03$ \\
\hline $5.50 \mathrm{E}-03$ & 0.826 & $9.42 \mathrm{E}+19$ & $9.73 \mathrm{E}-04$ & $8.92 \mathrm{E}+19$ & $9.21 \mathrm{E}-04$ & $8.63 E+19$ & 8.91E-04 & $8.08 \mathrm{E}+19$ & $8.34 \mathrm{E}-04$ \\
\hline $7.20 \mathrm{E}-03$ & 1.671 & $1.23 \mathrm{E}+20$ & 2.57E-03 & $1.15 \mathrm{E}+20$ & $2.41 \mathrm{E}-03$ & $1.09 \mathrm{E}+20$ & 2.27E-03 & $1.00 \mathrm{E}+20$ & 2.09E-03 \\
\hline $9.20 \mathrm{E}-03$ & 3.709 & $9.75 E+19$ & $4.52 \mathrm{E}-03$ & $9.48 \mathrm{E}+19$ & 4.39E-03 & $8.97 E+19$ & 4.16E-03 & $8.13 E+19$ & 3.77E-03 \\
\hline $1.20 \mathrm{E}-02$ & 1.491 & $1.15 \mathrm{E}+20$ & 2.15E-03 & $1.13 \mathrm{E}+20$ & $2.10 \mathrm{E}-03$ & $1.07 \mathrm{E}+20$ & $1.99 \mathrm{E}-03$ & $1.01 \mathrm{E}+20$ & $1.89 \mathrm{E}-03$ \\
\hline $1.50 \mathrm{E}-02$ & 1.156 & $1.02 \mathrm{E}+20$ & $1.48 \mathrm{E}-03$ & $9.69 \mathrm{E}+19$ & $1.40 \mathrm{E}-03$ & $9.35 \mathrm{E}+19$ & $1.35 \mathrm{E}-03$ & $8.80 \mathrm{E}+19$ & $1.27 \mathrm{E}-03$ \\
\hline $1.90 \mathrm{E}-02$ & 0.973 & $1.13 \mathrm{E}+20$ & $1.38 \mathrm{E}-03$ & $1.05 \mathrm{E}+20$ & 1.27E-03 & $9.83 \mathrm{E}+19$ & $1.20 \mathrm{E}-03$ & $8.98 \mathrm{E}+19$ & $1.09 \mathrm{E}-03$ \\
\hline $2.55 \mathrm{E}-02$ & 0.555 & $1.62 \mathrm{E}+20$ & $1.12 \mathrm{E}-03$ & $1.52 \mathrm{E}+20$ & $1.05 \mathrm{E}-03$ & $1.45 \mathrm{E}+20$ & $1.00 \mathrm{E}-03$ & $1.35 \mathrm{E}+20$ & $9.39 \mathrm{E}-04$ \\
\hline $3.20 \mathrm{E}-02$ & 26.762 & $1.45 \mathrm{E}+20$ & 4.85E-02 & $1.40 \mathrm{E}+20$ & $4.68 \mathrm{E}-02$ & $1.30 \mathrm{E}+20$ & 4.35E-02 & $1.23 \mathrm{E}+20$ & $4.11 \mathrm{E}-02$ \\
\hline $4.00 \mathrm{E}-02$ & 8.161 & $6.70 \mathrm{E}+19$ & $6.84 \mathrm{E}-03$ & $6.60 \mathrm{E}+19$ & $6.74 \mathrm{E}-03$ & $6.17 \mathrm{E}+19$ & $6.30 \mathrm{E}-03$ & $6.11 \mathrm{E}+19$ & $6.23 \mathrm{E}-03$ \\
\hline $5.25 \mathrm{E}-02$ & 6.515 & $1.41 \mathrm{E}+20$ & 1.15E-02 & $1.33 \mathrm{E}+20$ & $1.08 \mathrm{E}-02$ & $1.28 \mathrm{E}+20$ & 1.05E-02 & 1.17E+20 & 9.57E-03 \\
\hline $6.60 \mathrm{E}-02$ & 6.576 & $1.38 \mathrm{E}+20$ & $1.14 \mathrm{E}-02$ & $1.31 \mathrm{E}+20$ & $1.08 \mathrm{E}-02$ & $1.27 \mathrm{E}+20$ & 1.05E-02 & $1.15 \mathrm{E}+20$ & $9.45 \mathrm{E}-03$ \\
\hline $8.80 \mathrm{E}-02$ & 13.137 & $2.08 \mathrm{E}+20$ & $3.41 \mathrm{E}-02$ & $1.98 \mathrm{E}+20$ & $3.26 \mathrm{E}-02$ & $1.84 \mathrm{E}+20$ & $3.02 \mathrm{E}-02$ & $1.73 \mathrm{E}+20$ & $2.84 \mathrm{E}-02$ \\
\hline $1.10 \mathrm{E}-01$ & 11.84 & $1.28 \mathrm{E}+20$ & $1.89 \mathrm{E}-02$ & $1.19 \mathrm{E}+20$ & $1.76 \mathrm{E}-02$ & $1.15 \mathrm{E}+20$ & $1.70 \mathrm{E}-02$ & $1.08 \mathrm{E}+20$ & $1.60 \mathrm{E}-02$ \\
\hline $1.35 \mathrm{E}-01$ & 11.27 & $1.91 \mathrm{E}+20$ & $2.70 \mathrm{E}-02$ & $1.81 \mathrm{E}+20$ & $2.56 \mathrm{E}-02$ & $1.62 \mathrm{E}+20$ & $2.28 \mathrm{E}-02$ & $1.53 \mathrm{E}+20$ & $2.15 \mathrm{E}-02$ \\
\hline $1.60 \mathrm{E}-01$ & 21.53 & $1.12 \mathrm{E}+20$ & $3.00 \mathrm{E}-02$ & $1.08 \mathrm{E}+20$ & $2.91 \mathrm{E}-02$ & $1.02 \mathrm{E}+20$ & $2.74 \mathrm{E}-02$ & $9.36 \mathrm{E}+19$ & $2.52 \mathrm{E}-02$ \\
\hline $1.90 \mathrm{E}-01$ & 15.85 & $1.59 \mathrm{E}+20$ & $3.16 \mathrm{E}-02$ & $1.45 \mathrm{E}+20$ & $2.87 \mathrm{E}-02$ & $1.43 \mathrm{E}+20$ & $2.83 \mathrm{E}-02$ & $1.33 \mathrm{E}+20$ & 2.63E-02 \\
\hline $2.20 \mathrm{E}-01$ & 22.01 & $1.22 \mathrm{E}+20$ & 3.34E-02 & $1.21 \mathrm{E}+20$ & $3.33 \mathrm{E}-02$ & $1.10 \mathrm{E}+20$ & $3.02 \mathrm{E}-02$ & $1.04 \mathrm{E}+20$ & $2.86 \mathrm{E}-02$ \\
\hline
\end{tabular}




\begin{tabular}{|c|c|c|c|c|c|c|c|c|c|}
\hline \multirow[b]{2}{*}{$\begin{array}{c}\text { Upper } \\
\text { Energy(MeV) }\end{array}$} & \multirow{2}{*}{$\begin{array}{l}\text { DPA xsec } \\
\text { keV-barn }\end{array}$} & \multicolumn{2}{|c|}{$2-7$} & \multicolumn{2}{|c|}{$2-6$} & \multicolumn{2}{|c|}{$2-5$} & \multicolumn{2}{|c|}{$2-4$} \\
\hline & & Total Fluence & DPA & Total Fluence & DPA & Total Fluence & DPA & Total Fluence & DPA \\
\hline $2.55 \mathrm{E}-01$ & 18.27 & $1.44 \mathrm{E}+20$ & $3.28 \mathrm{E}-02$ & $1.36 \mathrm{E}+20$ & $3.10 \mathrm{E}-02$ & $1.33 E+20$ & 3.03E-02 & $1.25 \mathrm{E}+20$ & $2.86 \mathrm{E}-02$ \\
\hline $2.90 \mathrm{E}-01$ & 17.37 & $1.55 \mathrm{E}+20$ & 3.37E-02 & $1.50 \mathrm{E}+20$ & $3.26 \mathrm{E}-02$ & $1.43 \mathrm{E}+20$ & $3.11 \mathrm{E}-02$ & $1.35 \mathrm{E}+20$ & $2.92 \mathrm{E}-02$ \\
\hline $3.20 \mathrm{E}-01$ & 13.51 & $1.12 \mathrm{E}+20$ & 1.90E-02 & $1.04 \mathrm{E}+20$ & $1.75 \mathrm{E}-02$ & $1.01 \mathrm{E}+20$ & $1.71 \mathrm{E}-02$ & $8.86 \mathrm{E}+19$ & $1.50 \mathrm{E}-02$ \\
\hline $3.60 \mathrm{E}-01$ & 25.14 & $1.58 \mathrm{E}+20$ & 4.97E-02 & $1.50 \mathrm{E}+20$ & 4.70E-02 & $1.41 \mathrm{E}+20$ & 4.44E-02 & $1.30 \mathrm{E}+20$ & 4.07E-02 \\
\hline $4.00 \mathrm{E}-01$ & 46.52 & $1.35 \mathrm{E}+20$ & 7.83E-02 & $1.31 \mathrm{E}+20$ & 7.60E-02 & $1.14 \mathrm{E}+20$ & $6.62 \mathrm{E}-02$ & $1.13 \mathrm{E}+20$ & $6.60 \mathrm{E}-02$ \\
\hline $4.50 \mathrm{E}-01$ & 43.04 & $1.31 \mathrm{E}+20$ & 7.03E-02 & $1.23 \mathrm{E}+20$ & $6.62 \mathrm{E}-02$ & $1.14 \mathrm{E}+20$ & $6.14 \mathrm{E}-02$ & $1.06 \mathrm{E}+20$ & $5.72 \mathrm{E}-02$ \\
\hline $5.00 \mathrm{E}-01$ & 36.4 & $1.43 \mathrm{E}+20$ & $6.51 \mathrm{E}-02$ & $1.39 \mathrm{E}+20$ & 6.34E-02 & 1.27E+20 & 5.77E-02 & $1.22 \mathrm{E}+20$ & $5.56 \mathrm{E}-02$ \\
\hline $5.50 \mathrm{E}-01$ & 33.22 & $1.42 \mathrm{E}+20$ & $5.88 \mathrm{E}-02$ & $1.36 \mathrm{E}+20$ & $5.65 \mathrm{E}-02$ & $1.27 \mathrm{E}+20$ & 5.27E-02 & $1.22 \mathrm{E}+20$ & $5.08 \mathrm{E}-02$ \\
\hline $6.00 \mathrm{E}-01$ & 34.18 & $1.37 \mathrm{E}+20$ & $5.84 \mathrm{E}-02$ & $1.32 \mathrm{E}+20$ & 5.64E-02 & $1.25 \mathrm{E}+20$ & $5.32 \mathrm{E}-02$ & $1.19 \mathrm{E}+20$ & 5.09E-02 \\
\hline $6.60 \mathrm{E}-01$ & 19.64 & $1.68 \mathrm{E}+20$ & 4.13E-02 & $1.54 \mathrm{E}+20$ & 3.77E-02 & $1.49 \mathrm{E}+20$ & 3.67E-02 & $1.50 \mathrm{E}+20$ & $3.68 \mathrm{E}-02$ \\
\hline $7.20 \mathrm{E}-01$ & 48.89 & $1.71 \mathrm{E}+20$ & $1.04 \mathrm{E}-01$ & $1.72 \mathrm{E}+20$ & $1.05 \mathrm{E}-01$ & $1.56 \mathrm{E}+20$ & $9.53 \mathrm{E}-02$ & $1.50 \mathrm{E}+20$ & $9.19 \mathrm{E}-02$ \\
\hline $7.80 \mathrm{E}-01$ & 74.22 & $1.65 \mathrm{E}+20$ & $1.53 \mathrm{E}-01$ & $1.58 \mathrm{E}+20$ & $1.46 \mathrm{E}-01$ & $1.47 \mathrm{E}+20$ & 1.37E-01 & $1.37 \mathrm{E}+20$ & $1.28 \mathrm{E}-01$ \\
\hline $8.40 \mathrm{E}-01$ & 44.02 & $1.28 \mathrm{E}+20$ & 7.03E-02 & $1.23 \mathrm{E}+20$ & $6.79 \mathrm{E}-02$ & $1.15 \mathrm{E}+20$ & $6.33 \mathrm{E}-02$ & $1.07 \mathrm{E}+20$ & $5.86 \mathrm{E}-02$ \\
\hline $9.20 \mathrm{E}-01$ & 40.97 & $1.57 \mathrm{E}+20$ & $8.02 \mathrm{E}-02$ & $1.48 \mathrm{E}+20$ & 7.57E-02 & $1.37 \mathrm{E}+20$ & 6.99E-02 & $1.31 \mathrm{E}+20$ & $6.69 \mathrm{E}-02$ \\
\hline $1.00 \mathrm{E}+00$ & 50.95 & $1.47 \mathrm{E}+20$ & $9.35 \mathrm{E}-02$ & $1.34 \mathrm{E}+20$ & $8.51 \mathrm{E}-02$ & $1.22 \mathrm{E}+20$ & 7.79E-02 & $1.18 \mathrm{E}+20$ & 7.49E-02 \\
\hline $1.20 \mathrm{E}+00$ & 50.09 & $3.29 \mathrm{E}+20$ & $2.06 \mathrm{E}-01$ & $3.13 \mathrm{E}+20$ & 1.96E-01 & $2.91 \mathrm{E}+20$ & $1.82 \mathrm{E}-01$ & $2.82 \mathrm{E}+20$ & $1.76 \mathrm{E}-01$ \\
\hline $1.40 \mathrm{E}+00$ & 64.54 & $2.99 \mathrm{E}+20$ & 2.41E-01 & $2.80 \mathrm{E}+20$ & $2.26 \mathrm{E}-01$ & $2.65 \mathrm{E}+20$ & $2.14 \mathrm{E}-01$ & $2.38 \mathrm{E}+20$ & $1.92 \mathrm{E}-01$ \\
\hline $1.60 \mathrm{E}+00$ & 73.58 & $2.60 \mathrm{E}+20$ & 2.39E-01 & $2.48 \mathrm{E}+20$ & $2.28 \mathrm{E}-01$ & $2.39 \mathrm{E}+20$ & $2.20 \mathrm{E}-01$ & $2.15 E+20$ & $1.98 \mathrm{E}-01$ \\
\hline $1.80 \mathrm{E}+00$ & 76.46 & $2.23 \mathrm{E}+20$ & 2.14E-01 & $2.07 \mathrm{E}+20$ & $1.98 \mathrm{E}-01$ & $1.96 \mathrm{E}+20$ & $1.88 \mathrm{E}-01$ & $1.91 \mathrm{E}+20$ & $1.82 \mathrm{E}-01$ \\
\hline $2.00 \mathrm{E}+00$ & 95.15 & $1.97 \mathrm{E}+20$ & $2.35 \mathrm{E}-01$ & $1.88 \mathrm{E}+20$ & $2.23 \mathrm{E}-01$ & $1.72 \mathrm{E}+20$ & 2.05E-01 & $1.66 \mathrm{E}+20$ & 1.97E-01 \\
\hline $2.30 \mathrm{E}+00$ & 93.75 & $2.45 \mathrm{E}+20$ & 2.87E-01 & $2.29 \mathrm{E}+20$ & $2.68 \mathrm{E}-01$ & $2.12 \mathrm{E}+20$ & $2.48 \mathrm{E}-01$ & $2.02 \mathrm{E}+20$ & 2.37E-01 \\
\hline $2.60 \mathrm{E}+00$ & 112.05 & $2.30 \mathrm{E}+20$ & $3.23 \mathrm{E}-01$ & $2.05 E+20$ & 2.87E-01 & $2.03 E+20$ & $2.84 \mathrm{E}-01$ & $1.88 \mathrm{E}+20$ & $2.63 \mathrm{E}-01$ \\
\hline $2.90 \mathrm{E}+00$ & 123.55 & $1.71 \mathrm{E}+20$ & $2.64 \mathrm{E}-01$ & $1.60 \mathrm{E}+20$ & 2.47E-01 & $1.54 \mathrm{E}+20$ & $2.39 \mathrm{E}-01$ & $1.43 \mathrm{E}+20$ & $2.20 \mathrm{E}-01$ \\
\hline $3.30 \mathrm{E}+00$ & 133.45 & $1.73 \mathrm{E}+20$ & 2.89E-01 & $1.60 \mathrm{E}+20$ & 2.66E-01 & $1.56 \mathrm{E}+20$ & $2.60 \mathrm{E}-01$ & $1.36 \mathrm{E}+20$ & $2.26 \mathrm{E}-01$ \\
\hline $3.70 \mathrm{E}+00$ & 135.25 & $1.14 \mathrm{E}+20$ & $1.92 \mathrm{E}-01$ & $1.12 \mathrm{E}+20$ & $1.89 \mathrm{E}-01$ & $1.04 \mathrm{E}+20$ & $1.76 \mathrm{E}-01$ & $1.02 \mathrm{E}+20$ & $1.72 \mathrm{E}-01$ \\
\hline $4.10 \mathrm{E}+00$ & 149.55 & $8.87 \mathrm{E}+19$ & $1.66 \mathrm{E}-01$ & $8.54 \mathrm{E}+19$ & $1.60 \mathrm{E}-01$ & $8.09 \mathrm{E}+19$ & $1.51 \mathrm{E}-01$ & $7.46 \mathrm{E}+19$ & $1.39 \mathrm{E}-01$ \\
\hline $4.50 \mathrm{E}+00$ & 158.25 & $6.86 \mathrm{E}+19$ & $1.36 \mathrm{E}-01$ & $6.53 E+19$ & 1.29E-01 & $6.31 \mathrm{E}+19$ & $1.25 \mathrm{E}-01$ & $5.87 \mathrm{E}+19$ & $1.16 \mathrm{E}-01$ \\
\hline $5.00 \mathrm{E}+00$ & 168.55 & $6.76 \mathrm{E}+19$ & $1.42 \mathrm{E}-01$ & $6.01 \mathrm{E}+19$ & 1.27E-01 & $5.94 \mathrm{E}+19$ & $1.25 \mathrm{E}-01$ & $5.45 \mathrm{E}+19$ & 1.15E-01 \\
\hline $5.50 \mathrm{E}+00$ & 176.46 & $4.50 E+19$ & 9.94E-02 & 4.13E+19 & $9.11 \mathrm{E}-02$ & 4.29E+19 & $9.46 \mathrm{E}-02$ & 3.70E+19 & $8.16 \mathrm{E}-02$ \\
\hline
\end{tabular}




\begin{tabular}{|c|c|c|c|c|c|c|c|c|c|}
\hline \multirow{2}{*}{$\begin{array}{c}\text { Upper } \\
\text { Energy(MeV) }\end{array}$} & \multirow{2}{*}{$\begin{array}{l}\text { DPA xsec } \\
\text { keV-barn }\end{array}$} & \multicolumn{2}{|c|}{$2-7$} & \multicolumn{2}{|c|}{$2-6$} & \multicolumn{2}{|c|}{$2-5$} & \multicolumn{2}{|c|}{$2-4$} \\
\hline & & Total Fluence & DPA & Total Fluence & DPA & Total Fluence & DPA & Total Fluence & DPA \\
\hline $6.00 \mathrm{E}+00$ & 183.06 & $3.22 E+19$ & 7.37E-02 & $2.88 \mathrm{E}+19$ & $6.59 \mathrm{E}-02$ & $2.77 \mathrm{E}+19$ & $6.33 \mathrm{E}-02$ & $2.70 \mathrm{E}+19$ & $6.17 \mathrm{E}-02$ \\
\hline $6.70 \mathrm{E}+00$ & 189.26 & $2.89 \mathrm{E}+19$ & $6.83 \mathrm{E}-02$ & $2.61 E+19$ & $6.17 \mathrm{E}-02$ & $2.50 E+19$ & $5.91 \mathrm{E}-02$ & $2.34 \mathrm{E}+19$ & $5.54 \mathrm{E}-02$ \\
\hline $7.40 \mathrm{E}+00$ & 196.67 & $1.51 \mathrm{E}+19$ & 3.72E-02 & $1.55 \mathrm{E}+19$ & $3.81 \mathrm{E}-02$ & $1.32 \mathrm{E}+19$ & $3.23 \mathrm{E}-02$ & $1.30 \mathrm{E}+19$ & $3.20 \mathrm{E}-02$ \\
\hline $8.20 \mathrm{E}+00$ & 203.37 & $1.02 \mathrm{E}+19$ & $2.60 \mathrm{E}-02$ & $1.00 \mathrm{E}+19$ & $2.55 \mathrm{E}-02$ & $1.06 \mathrm{E}+19$ & $2.70 \mathrm{E}-02$ & $8.15 \mathrm{E}+18$ & 2.07E-02 \\
\hline $9.00 \mathrm{E}+00$ & 214.58 & $6.42 \mathrm{E}+18$ & $1.72 \mathrm{E}-02$ & $4.75 \mathrm{E}+18$ & $1.27 \mathrm{E}-02$ & $5.41 \mathrm{E}+18$ & $1.45 \mathrm{E}-02$ & $3.13 \mathrm{E}+18$ & $8.40 \mathrm{E}-03$ \\
\hline $1.00 \mathrm{E}+01$ & 225.69 & $3.25 E+18$ & $9.18 \mathrm{E}-03$ & $3.65 \mathrm{E}+18$ & $1.03 \mathrm{E}-02$ & $3.13 \mathrm{E}+18$ & $8.84 \mathrm{E}-03$ & $3.14 \mathrm{E}+18$ & $8.86 \mathrm{E}-03$ \\
\hline $1.10 \mathrm{E}+01$ & 237.6 & $1.57 \mathrm{E}+18$ & 4.66E-03 & $1.26 \mathrm{E}+18$ & $3.76 \mathrm{E}-03$ & $1.83 \mathrm{E}+18$ & $5.43 \mathrm{E}-03$ & $1.70 \mathrm{E}+18$ & $5.04 \mathrm{E}-03$ \\
\hline $1.20 \mathrm{E}+01$ & 247.41 & $5.99 \mathrm{E}+17$ & $1.85 \mathrm{E}-03$ & $5.31 \mathrm{E}+17$ & $1.64 \mathrm{E}-03$ & $8.21 \mathrm{E}+17$ & $2.54 \mathrm{E}-03$ & $9.50 \mathrm{E}+17$ & $2.94 \mathrm{E}-03$ \\
\hline $1.30 \mathrm{E}+01$ & 258.52 & $1.11 \mathrm{E}+17$ & $3.58 \mathrm{E}-04$ & $2.81 \mathrm{E}+17$ & $9.09 \mathrm{E}-04$ & $2.35 \mathrm{E}+17$ & $7.58 \mathrm{E}-04$ & $2.64 \mathrm{E}+17$ & $8.53 \mathrm{E}-04$ \\
\hline $1.40 \mathrm{E}+01$ & 271.35 & $1.83 \mathrm{E}+17$ & $6.20 \mathrm{E}-04$ & $1.57 \mathrm{E}+17$ & $5.32 \mathrm{E}-04$ & $1.48 \mathrm{E}+17$ & $5.01 \mathrm{E}-04$ & $1.18 \mathrm{E}+17$ & $3.99 \mathrm{E}-04$ \\
\hline $1.50 \mathrm{E}+01$ & 290.27 & $6.98 \mathrm{E}+16$ & $2.53 \mathrm{E}-04$ & $7.19 E+16$ & $2.61 \mathrm{E}-04$ & $0.00 \mathrm{E}+00$ & $0.00 \mathrm{E}+00$ & $4.64 \mathrm{E}+16$ & $1.68 \mathrm{E}-04$ \\
\hline $1.60 \mathrm{E}+01$ & 293.2 & $0.00 \mathrm{E}+00$ & $0.00 \mathrm{E}+00$ & $5.36 \mathrm{E}+16$ & $1.97 \mathrm{E}-04$ & $0.00 \mathrm{E}+00$ & $0.00 \mathrm{E}+00$ & $0.00 \mathrm{E}+00$ & $0.00 \mathrm{E}+00$ \\
\hline $1.70 \mathrm{E}+01$ & 292.73 & $0.00 E+00$ & $0.00 E+00$ & $0.00 \mathrm{E}+00$ & $0.00 \mathrm{E}+00$ & $0.00 \mathrm{E}+00$ & $0.00 \mathrm{E}+00$ & $0.00 \mathrm{E}+00$ & $0.00 \mathrm{E}+00$ \\
\hline $1.80 \mathrm{E}+01$ & 297.65 & $0.00 \mathrm{E}+00$ & $0.00 \mathrm{E}+00$ & $0.00 \mathrm{E}+00$ & $0.00 \mathrm{E}+00$ & $1.08 \mathrm{E}+16$ & 4.03E-05 & $6.63 \mathrm{E}+16$ & 2.47E-04 \\
\hline $1.90 \mathrm{E}+01$ & 307.26 & $0.00 \mathrm{E}+00$ & $0.00 \mathrm{E}+00$ & $0.00 \mathrm{E}+00$ & $0.00 \mathrm{E}+00$ & $0.00 \mathrm{E}+00$ & $0.00 \mathrm{E}+00$ & $0.00 \mathrm{E}+00$ & $0.00 \mathrm{E}+00$ \\
\hline \multirow[t]{2}{*}{$2.00 \mathrm{E}+01$} & 316.36 & $4.23 E+16$ & 1.67E-04 & $0.00 \mathrm{E}+00$ & $0.00 E+00$ & $0.00 \mathrm{E}+00$ & $0.00 \mathrm{E}+00$ & $0.00 \mathrm{E}+00$ & $0.00 E+00$ \\
\hline & & & $4.58 E+00$ & & $4.30 E+00$ & & $4.09 E+00$ & & $3.81 E+00$ \\
\hline
\end{tabular}


Table 20. Calculated fluence and DPA for capsule 2 of the UCSB experiment.

\begin{tabular}{|c|c|c|c|c|c|c|c|}
\hline \multirow{2}{*}{$\begin{array}{c}\text { Upper } \\
\text { Energy(MeV) }\end{array}$} & \multirow{2}{*}{$\begin{array}{l}\text { DPA xsec } \\
\text { keV-barn }\end{array}$} & \multicolumn{2}{|c|}{$2-3$} & \multicolumn{2}{|c|}{$2-2$} & \multicolumn{2}{|c|}{$2-1$} \\
\hline & & Total Fluence & DPA & Total Fluence & DPA & Total Fluence & DPA \\
\hline $1.00 \mathrm{E}-10$ & $0.00 E+00$ & $7.39 E+14$ & & $0.00 E+00$ & & $2.35 \mathrm{E}+15$ & \\
\hline $1.00 \mathrm{E}-09$ & $7.73 \mathrm{E}+00$ & $3.61 \mathrm{E}+17$ & $3.49 \mathrm{E}-05$ & $1.93 \mathrm{E}+17$ & $1.87 \mathrm{E}-05$ & $2.46 \mathrm{E}+17$ & 2.37E-05 \\
\hline $1.00 \mathrm{E}-08$ & $2.44 \mathrm{E}+00$ & $5.93 E+19$ & $1.81 \mathrm{E}-03$ & $5.36 \mathrm{E}+19$ & $1.64 \mathrm{E}-03$ & $4.96 \mathrm{E}+19$ & $1.52 \mathrm{E}-03$ \\
\hline $2.30 \mathrm{E}-08$ & $1.28 \mathrm{E}+00$ & $2.12 \mathrm{E}+20$ & 3.39E-03 & $1.94 \mathrm{E}+20$ & $3.10 \mathrm{E}-03$ & $1.79 \mathrm{E}+20$ & $2.86 \mathrm{E}-03$ \\
\hline $5.00 \mathrm{E}-08$ & $8.57 \mathrm{E}-01$ & $5.24 \mathrm{E}+20$ & $5.61 \mathrm{E}-03$ & $4.76 \mathrm{E}+20$ & $5.10 \mathrm{E}-03$ & $4.43 \mathrm{E}+20$ & 4.75E-03 \\
\hline $7.60 \mathrm{E}-08$ & $6.44 \mathrm{E}-01$ & $3.76 \mathrm{E}+20$ & 3.03E-03 & $3.43 E+20$ & $2.76 \mathrm{E}-03$ & $3.17 \mathrm{E}+20$ & $2.55 \mathrm{E}-03$ \\
\hline $1.15 \mathrm{E}-07$ & 5.23E-01 & $3.29 \mathrm{E}+20$ & $2.15 \mathrm{E}-03$ & $3.02 \mathrm{E}+20$ & $1.97 \mathrm{E}-03$ & $2.79 \mathrm{E}+20$ & $1.83 \mathrm{E}-03$ \\
\hline $1.70 \mathrm{E}-07$ & $4.28 \mathrm{E}-01$ & $2.14 \mathrm{E}+20$ & $1.15 \mathrm{E}-03$ & $1.96 \mathrm{E}+20$ & $1.05 \mathrm{E}-03$ & $1.83 \mathrm{E}+20$ & $9.78 \mathrm{E}-04$ \\
\hline $2.55 \mathrm{E}-07$ & $3.51 \mathrm{E}-01$ & $1.55 \mathrm{E}+20$ & $6.81 \mathrm{E}-04$ & $1.40 \mathrm{E}+20$ & $6.15 \mathrm{E}-04$ & $1.29 \mathrm{E}+20$ & $5.68 \mathrm{E}-04$ \\
\hline $3.80 \mathrm{E}-07$ & $2.87 \mathrm{E}-01$ & $1.26 \mathrm{E}+20$ & 4.53E-04 & $1.16 \mathrm{E}+20$ & $4.18 \mathrm{E}-04$ & $1.14 \mathrm{E}+20$ & 4.10E-04 \\
\hline $5.50 \mathrm{E}-07$ & 2.37E-01 & $1.18 \mathrm{E}+20$ & 3.50E-04 & $1.06 \mathrm{E}+20$ & $3.15 \mathrm{E}-04$ & $1.00 \mathrm{E}+20$ & 2.97E-04 \\
\hline $8.40 \mathrm{E}-07$ & $1.94 \mathrm{E}-01$ & $1.37 \mathrm{E}+20$ & $3.31 \mathrm{E}-04$ & $1.26 \mathrm{E}+20$ & $3.06 \mathrm{E}-04$ & $1.09 \mathrm{E}+20$ & $2.64 \mathrm{E}-04$ \\
\hline $1.28 \mathrm{E}-06$ & $1.57 \mathrm{E}-01$ & $1.30 \mathrm{E}+20$ & $2.55 \mathrm{E}-04$ & 1.17E+20 & $2.29 \mathrm{E}-04$ & $1.14 \mathrm{E}+20$ & $2.24 \mathrm{E}-04$ \\
\hline $1.90 \mathrm{E}-06$ & $1.28 \mathrm{E}-01$ & $1.18 \mathrm{E}+20$ & $1.89 \mathrm{E}-04$ & $1.11 \mathrm{E}+20$ & $1.77 \mathrm{E}-04$ & $1.02 \mathrm{E}+20$ & 1.64E-04 \\
\hline $2.80 \mathrm{E}-06$ & $1.05 \mathrm{E}-01$ & $1.16 \mathrm{E}+20$ & $1.53 \mathrm{E}-04$ & $1.06 \mathrm{E}+20$ & $1.39 \mathrm{E}-04$ & $1.02 \mathrm{E}+20$ & $1.34 \mathrm{E}-04$ \\
\hline 4.25E-06 & $8.60 \mathrm{E}-02$ & $1.23 \mathrm{E}+20$ & $1.32 \mathrm{E}-04$ & $1.11 \mathrm{E}+20$ & 1.19E-04 & $1.07 \mathrm{E}+20$ & 1.16E-04 \\
\hline $6.30 \mathrm{E}-06$ & $7.00 \mathrm{E}-02$ & $1.14 \mathrm{E}+20$ & $9.93 \mathrm{E}-05$ & $1.05 \mathrm{E}+20$ & $9.16 \mathrm{E}-05$ & $9.74 \mathrm{E}+19$ & 8.53E-05 \\
\hline $9.20 \mathrm{E}-06$ & $5.80 \mathrm{E}-02$ & $1.09 \mathrm{E}+20$ & 7.90E-05 & $1.03 \mathrm{E}+20$ & 7.47E-05 & $9.46 \mathrm{E}+19$ & $6.86 \mathrm{E}-05$ \\
\hline $1.35 \mathrm{E}-05$ & $4.80 \mathrm{E}-02$ & $1.15 \mathrm{E}+20$ & $6.90 \mathrm{E}-05$ & $1.10 \mathrm{E}+20$ & $6.57 \mathrm{E}-05$ & $9.76 \mathrm{E}+19$ & $5.86 \mathrm{E}-05$ \\
\hline $2.10 \mathrm{E}-05$ & $3.80 \mathrm{E}-02$ & $1.31 \mathrm{E}+20$ & $6.21 \mathrm{E}-05$ & $1.18 \mathrm{E}+20$ & 5.61E-05 & $1.10 \mathrm{E}+20$ & $5.22 \mathrm{E}-05$ \\
\hline $3.00 \mathrm{E}-05$ & $3.10 \mathrm{E}-02$ & $1.08 \mathrm{E}+20$ & 4.19E-05 & $1.02 \mathrm{E}+20$ & 3.97E-05 & $9.75 E+19$ & $3.78 \mathrm{E}-05$ \\
\hline $4.50 \mathrm{E}-05$ & $2.60 \mathrm{E}-02$ & $1.34 \mathrm{E}+20$ & 4.35E-05 & $1.21 \mathrm{E}+20$ & $3.94 \mathrm{E}-05$ & $1.11 \mathrm{E}+20$ & $3.61 \mathrm{E}-05$ \\
\hline $6.90 \mathrm{E}-05$ & $2.10 \mathrm{E}-02$ & $1.34 \mathrm{E}+20$ & 3.51E-05 & $1.21 \mathrm{E}+20$ & $3.18 \mathrm{E}-05$ & $1.18 \mathrm{E}+20$ & 3.10E-05 \\
\hline $1.00 \mathrm{E}-04$ & $1.70 \mathrm{E}-02$ & $1.20 \mathrm{E}+20$ & $2.54 \mathrm{E}-05$ & $1.12 \mathrm{E}+20$ & $2.39 \mathrm{E}-05$ & $1.02 \mathrm{E}+20$ & 2.16E-05 \\
\hline $1.35 \mathrm{E}-04$ & $1.40 \mathrm{E}-02$ & $9.58 \mathrm{E}+19$ & $1.68 \mathrm{E}-05$ & $8.98 E+19$ & $1.57 \mathrm{E}-05$ & $8.36 E+19$ & $1.46 \mathrm{E}-05$ \\
\hline $1.70 \mathrm{E}-04$ & $1.40 \mathrm{E}-02$ & $7.40 \mathrm{E}+19$ & $1.29 \mathrm{E}-05$ & $6.91 \mathrm{E}+19$ & $1.21 \mathrm{E}-05$ & $6.24 \mathrm{E}+19$ & $1.09 \mathrm{E}-05$ \\
\hline $2.20 \mathrm{E}-04$ & $1.10 \mathrm{E}-02$ & $8.71 E+19$ & $1.20 \mathrm{E}-05$ & $7.53 E+19$ & $1.03 \mathrm{E}-05$ & $7.18 \mathrm{E}+19$ & $9.88 \mathrm{E}-06$ \\
\hline
\end{tabular}




\begin{tabular}{|c|c|c|c|c|c|c|c|}
\hline \multirow{2}{*}{$\begin{array}{c}\text { Upper } \\
\text { Energy(MeV) }\end{array}$} & \multirow{2}{*}{$\begin{array}{l}\text { DPA xsec } \\
\text { keV-barn }\end{array}$} & \multicolumn{2}{|c|}{$2-3$} & \multicolumn{2}{|c|}{$2-2$} & \multicolumn{2}{|c|}{$2-1$} \\
\hline & & Total Fluence & DPA & Total Fluence & DPA & Total Fluence & DPA \\
\hline $2.80 \mathrm{E}-04$ & $1.10 \mathrm{E}-02$ & $8.13 E+19$ & $1.12 \mathrm{E}-05$ & $6.99 E+19$ & $9.61 \mathrm{E}-06$ & $6.71 \mathrm{E}+19$ & $9.22 \mathrm{E}-06$ \\
\hline $3.60 \mathrm{E}-04$ & $1.00 \mathrm{E}-02$ & $7.96 \mathrm{E}+19$ & $9.94 \mathrm{E}-06$ & $7.84 \mathrm{E}+19$ & $9.80 \mathrm{E}-06$ & $7.12 \mathrm{E}+19$ & $8.89 \mathrm{E}-06$ \\
\hline $4.50 \mathrm{E}-04$ & $7.00 \mathrm{E}-03$ & $7.11 \mathrm{E}+19$ & $6.22 \mathrm{E}-06$ & $6.70 \mathrm{E}+19$ & $5.86 \mathrm{E}-06$ & $6.55 \mathrm{E}+19$ & 5.73E-06 \\
\hline $5.75 \mathrm{E}-04$ & $5.00 \mathrm{E}-03$ & $8.51 \mathrm{E}+19$ & 5.32E-06 & $7.75 \mathrm{E}+19$ & $4.84 \mathrm{E}-06$ & $6.93 \mathrm{E}+19$ & 4.33E-06 \\
\hline $7.60 \mathrm{E}-04$ & $1.29 \mathrm{E}-01$ & $9.38 \mathrm{E}+19$ & $1.51 \mathrm{E}-04$ & $8.55 \mathrm{E}+19$ & $1.38 \mathrm{E}-04$ & $8.21 \mathrm{E}+19$ & $1.32 \mathrm{E}-04$ \\
\hline $9.60 \mathrm{E}-04$ & $3.08 \mathrm{E}-01$ & $7.80 E+19$ & 3.00E-04 & $7.06 \mathrm{E}+19$ & $2.72 \mathrm{E}-04$ & $6.49 E+19$ & $2.50 \mathrm{E}-04$ \\
\hline $1.28 \mathrm{E}-03$ & $6.72 \mathrm{E}-01$ & $9.26 \mathrm{E}+19$ & 7.78E-04 & $8.71 \mathrm{E}+19$ & $7.32 \mathrm{E}-04$ & $8.11 \mathrm{E}+19$ & $6.82 \mathrm{E}-04$ \\
\hline $1.60 \mathrm{E}-03$ & 4.77E-01 & $7.67 E+19$ & 4.57E-04 & $7.06 \mathrm{E}+19$ & $4.21 \mathrm{E}-04$ & $6.31 \mathrm{E}+19$ & $3.76 \mathrm{E}-04$ \\
\hline $2.00 \mathrm{E}-03$ & $5.11 \mathrm{E}-01$ & $7.61 \mathrm{E}+19$ & 4.86E-04 & $6.82 \mathrm{E}+19$ & $4.35 \mathrm{E}-04$ & $6.26 \mathrm{E}+19$ & 4.00E-04 \\
\hline $2.70 \mathrm{E}-03$ & $5.68 \mathrm{E}-01$ & $1.04 \mathrm{E}+20$ & $7.38 \mathrm{E}-04$ & $9.19 \mathrm{E}+19$ & $6.53 \mathrm{E}-04$ & $8.77 E+19$ & $6.23 \mathrm{E}-04$ \\
\hline $3.40 \mathrm{E}-03$ & $6.32 \mathrm{E}-01$ & $7.89 E+19$ & $6.24 \mathrm{E}-04$ & $7.65 E+19$ & $6.05 \mathrm{E}-04$ & $7.14 \mathrm{E}+19$ & 5.64E-04 \\
\hline $4.50 \mathrm{E}-03$ & $8.22 \mathrm{E}-01$ & $9.87 E+19$ & $1.01 \mathrm{E}-03$ & $8.96 \mathrm{E}+19$ & $9.20 \mathrm{E}-04$ & $8.40 \mathrm{E}+19$ & 8.63E-04 \\
\hline $5.50 \mathrm{E}-03$ & $8.26 \mathrm{E}-01$ & $7.28 \mathrm{E}+19$ & $7.52 \mathrm{E}-04$ & $6.64 \mathrm{E}+19$ & $6.86 \mathrm{E}-04$ & $6.37 E+19$ & $6.58 \mathrm{E}-04$ \\
\hline 7.20E-03 & $1.67 \mathrm{E}+00$ & $9.66 \mathrm{E}+19$ & $2.02 \mathrm{E}-03$ & $8.67 \mathrm{E}+19$ & $1.81 \mathrm{E}-03$ & $8.40 \mathrm{E}+19$ & $1.76 \mathrm{E}-03$ \\
\hline $9.20 \mathrm{E}-03$ & $3.71 E+00$ & $7.68 \mathrm{E}+19$ & 3.56E-03 & $7.27 \mathrm{E}+19$ & 3.37E-03 & $6.53 \mathrm{E}+19$ & 3.03E-03 \\
\hline $1.20 \mathrm{E}-02$ & $1.49 \mathrm{E}+00$ & $9.17 \mathrm{E}+19$ & $1.71 \mathrm{E}-03$ & $8.64 \mathrm{E}+19$ & $1.61 \mathrm{E}-03$ & $8.37 E+19$ & $1.56 \mathrm{E}-03$ \\
\hline $1.50 \mathrm{E}-02$ & $1.16 \mathrm{E}+00$ & $7.87 E+19$ & $1.14 \mathrm{E}-03$ & $7.17 E+19$ & $1.04 \mathrm{E}-03$ & $6.72 \mathrm{E}+19$ & $9.71 \mathrm{E}-04$ \\
\hline $1.90 \mathrm{E}-02$ & $9.73 \mathrm{E}-01$ & $8.38 \mathrm{E}+19$ & $1.02 \mathrm{E}-03$ & $8.02 E+19$ & $9.76 \mathrm{E}-04$ & $7.20 \mathrm{E}+19$ & 8.76E-04 \\
\hline $2.55 \mathrm{E}-02$ & $5.55 \mathrm{E}-01$ & $1.25 \mathrm{E}+20$ & 8.66E-04 & $1.11 \mathrm{E}+20$ & $7.72 \mathrm{E}-04$ & $1.03 \mathrm{E}+20$ & 7.11E-04 \\
\hline $3.20 \mathrm{E}-02$ & $2.68 \mathrm{E}+01$ & 1.17E+20 & 3.91E-02 & $1.02 \mathrm{E}+20$ & $3.42 \mathrm{E}-02$ & $9.81 \mathrm{E}+19$ & $3.28 \mathrm{E}-02$ \\
\hline $4.00 \mathrm{E}-02$ & $8.16 \mathrm{E}+00$ & $5.53 \mathrm{E}+19$ & 5.64E-03 & $5.13 \mathrm{E}+19$ & $5.23 \mathrm{E}-03$ & $4.31 \mathrm{E}+19$ & 4.40E-03 \\
\hline $5.25 \mathrm{E}-02$ & $6.52 \mathrm{E}+00$ & $1.06 \mathrm{E}+20$ & $8.62 \mathrm{E}-03$ & $1.04 \mathrm{E}+20$ & $8.45 \mathrm{E}-03$ & $9.30 \mathrm{E}+19$ & 7.57E-03 \\
\hline $6.60 \mathrm{E}-02$ & $6.58 \mathrm{E}+00$ & $1.06 \mathrm{E}+20$ & 8.72E-03 & $1.03 \mathrm{E}+20$ & $8.48 \mathrm{E}-03$ & $8.96 \mathrm{E}+19$ & 7.36E-03 \\
\hline $8.80 \mathrm{E}-02$ & $1.31 \mathrm{E}+01$ & $1.58 \mathrm{E}+20$ & $2.60 \mathrm{E}-02$ & $1.46 \mathrm{E}+20$ & $2.40 \mathrm{E}-02$ & $1.36 \mathrm{E}+20$ & $2.24 \mathrm{E}-02$ \\
\hline 1.10E-01 & $1.18 \mathrm{E}+01$ & $9.46 \mathrm{E}+19$ & $1.40 \mathrm{E}-02$ & $8.94 \mathrm{E}+19$ & $1.32 \mathrm{E}-02$ & $8.03 E+19$ & 1.19E-02 \\
\hline $1.35 \mathrm{E}-01$ & $1.13 \mathrm{E}+01$ & $1.46 \mathrm{E}+20$ & $2.05 \mathrm{E}-02$ & $1.32 \mathrm{E}+20$ & $1.86 \mathrm{E}-02$ & $1.24 \mathrm{E}+20$ & $1.74 \mathrm{E}-02$ \\
\hline $1.60 \mathrm{E}-01$ & $2.15 \mathrm{E}+01$ & $8.48 \mathrm{E}+19$ & $2.28 \mathrm{E}-02$ & $7.89 \mathrm{E}+19$ & $2.12 \mathrm{E}-02$ & $7.29 \mathrm{E}+19$ & $1.96 \mathrm{E}-02$ \\
\hline $1.90 \mathrm{E}-01$ & $1.59 \mathrm{E}+01$ & $1.18 \mathrm{E}+20$ & $2.34 \mathrm{E}-02$ & $1.04 \mathrm{E}+20$ & $2.05 \mathrm{E}-02$ & $9.68 \mathrm{E}+19$ & $1.92 \mathrm{E}-02$ \\
\hline $2.20 \mathrm{E}-01$ & $2.20 \mathrm{E}+01$ & $9.57 E+19$ & $2.63 \mathrm{E}-02$ & $8.83 E+19$ & $2.43 \mathrm{E}-02$ & $8.44 \mathrm{E}+19$ & $2.32 \mathrm{E}-02$ \\
\hline
\end{tabular}




\begin{tabular}{|c|c|c|c|c|c|c|c|}
\hline \multirow{2}{*}{$\begin{array}{c}\text { Upper } \\
\text { Energy(MeV) }\end{array}$} & \multirow{2}{*}{$\begin{array}{l}\text { DPA xsec } \\
\text { keV-barn }\end{array}$} & \multicolumn{2}{|c|}{$2-3$} & \multicolumn{2}{|c|}{$2-2$} & \multicolumn{2}{|c|}{$2-1$} \\
\hline & & Total Fluence & DPA & Total Fluence & DPA & Total Fluence & DPA \\
\hline $2.55 \mathrm{E}-01$ & $1.83 \mathrm{E}+01$ & $1.13 \mathrm{E}+20$ & $2.59 \mathrm{E}-02$ & $1.04 \mathrm{E}+20$ & $2.36 \mathrm{E}-02$ & $9.23 E+19$ & $2.11 \mathrm{E}-02$ \\
\hline $2.90 \mathrm{E}-01$ & $1.74 \mathrm{E}+01$ & $1.18 \mathrm{E}+20$ & $2.56 \mathrm{E}-02$ & $1.08 \mathrm{E}+20$ & $2.36 \mathrm{E}-02$ & $9.91 \mathrm{E}+19$ & $2.15 \mathrm{E}-02$ \\
\hline $3.20 \mathrm{E}-01$ & $1.35 \mathrm{E}+01$ & $8.52 \mathrm{E}+19$ & $1.44 \mathrm{E}-02$ & $7.82 \mathrm{E}+19$ & $1.32 \mathrm{E}-02$ & $6.82 \mathrm{E}+19$ & $1.15 \mathrm{E}-02$ \\
\hline $3.60 \mathrm{E}-01$ & $2.51 \mathrm{E}+01$ & $1.22 \mathrm{E}+20$ & $3.83 \mathrm{E}-02$ & $1.05 E+20$ & 3.31E-02 & $9.61 \mathrm{E}+19$ & $3.02 \mathrm{E}-02$ \\
\hline 4.00E-01 & $4.65 \mathrm{E}+01$ & $1.04 \mathrm{E}+20$ & 6.03E-02 & $9.12 \mathrm{E}+19$ & $5.30 \mathrm{E}-02$ & $8.41 \mathrm{E}+19$ & 4.89E-02 \\
\hline $4.50 \mathrm{E}-01$ & $4.30 \mathrm{E}+01$ & $9.55 E+19$ & $5.14 \mathrm{E}-02$ & $8.67 E+19$ & 4.67E-02 & $8.23 E+19$ & 4.43E-02 \\
\hline $5.00 \mathrm{E}-01$ & $3.64 \mathrm{E}+01$ & $1.11 \mathrm{E}+20$ & 5.05E-02 & $1.00 \mathrm{E}+20$ & 4.57E-02 & $9.28 \mathrm{E}+19$ & $4.22 \mathrm{E}-02$ \\
\hline $5.50 \mathrm{E}-01$ & $3.32 \mathrm{E}+01$ & $1.07 \mathrm{E}+20$ & 4.43E-02 & $9.82 \mathrm{E}+19$ & $4.08 \mathrm{E}-02$ & $9.18 \mathrm{E}+19$ & $3.81 \mathrm{E}-02$ \\
\hline $6.00 \mathrm{E}-01$ & $3.42 \mathrm{E}+01$ & $1.03 \mathrm{E}+20$ & $4.41 \mathrm{E}-02$ & $9.58 \mathrm{E}+19$ & 4.09E-02 & $8.69 \mathrm{E}+19$ & $3.71 \mathrm{E}-02$ \\
\hline $6.60 \mathrm{E}-01$ & $1.96 \mathrm{E}+01$ & $1.28 \mathrm{E}+20$ & 3.14E-02 & $1.21 \mathrm{E}+20$ & $2.98 \mathrm{E}-02$ & $1.06 \mathrm{E}+20$ & $2.61 \mathrm{E}-02$ \\
\hline 7.20E-01 & $4.89 \mathrm{E}+01$ & $1.31 \mathrm{E}+20$ & 8.00E-02 & $1.20 \mathrm{E}+20$ & 7.32E-02 & $1.12 \mathrm{E}+20$ & $6.86 \mathrm{E}-02$ \\
\hline $7.80 \mathrm{E}-01$ & $7.42 \mathrm{E}+01$ & $1.22 \mathrm{E}+20$ & $1.13 \mathrm{E}-01$ & $1.07 E+20$ & $9.94 \mathrm{E}-02$ & $1.11 \mathrm{E}+20$ & $1.03 \mathrm{E}-01$ \\
\hline $8.40 \mathrm{E}-01$ & $4.40 \mathrm{E}+01$ & $1.01 \mathrm{E}+20$ & $5.56 \mathrm{E}-02$ & $8.79 \mathrm{E}+19$ & 4.84E-02 & $8.21 \mathrm{E}+19$ & $4.52 \mathrm{E}-02$ \\
\hline $9.20 \mathrm{E}-01$ & $4.10 \mathrm{E}+01$ & $1.17 \mathrm{E}+20$ & 5.99E-02 & $1.07 \mathrm{E}+20$ & $5.50 \mathrm{E}-02$ & $9.89 \mathrm{E}+19$ & 5.07E-02 \\
\hline $1.00 \mathrm{E}+00$ & $5.10 \mathrm{E}+01$ & $1.08 \mathrm{E}+20$ & 6.90E-02 & $9.87 E+19$ & $6.29 \mathrm{E}-02$ & $9.43 E+19$ & $6.01 \mathrm{E}-02$ \\
\hline $1.20 \mathrm{E}+00$ & $5.01 \mathrm{E}+01$ & $2.56 \mathrm{E}+20$ & $1.60 \mathrm{E}-01$ & $2.26 \mathrm{E}+20$ & $1.42 \mathrm{E}-01$ & $2.15 E+20$ & $1.35 \mathrm{E}-01$ \\
\hline $1.40 \mathrm{E}+00$ & $6.45 \mathrm{E}+01$ & $2.25 \mathrm{E}+20$ & $1.81 \mathrm{E}-01$ & $2.00 \mathrm{E}+20$ & $1.62 \mathrm{E}-01$ & $1.91 \mathrm{E}+20$ & $1.54 \mathrm{E}-01$ \\
\hline $1.60 \mathrm{E}+00$ & $7.36 \mathrm{E}+01$ & $1.93 \mathrm{E}+20$ & $1.78 \mathrm{E}-01$ & $1.72 \mathrm{E}+20$ & $1.58 \mathrm{E}-01$ & $1.67 \mathrm{E}+20$ & $1.54 \mathrm{E}-01$ \\
\hline $1.80 \mathrm{E}+00$ & $7.65 \mathrm{E}+01$ & $1.74 \mathrm{E}+20$ & $1.66 \mathrm{E}-01$ & $1.48 \mathrm{E}+20$ & $1.41 \mathrm{E}-01$ & $1.39 \mathrm{E}+20$ & $1.33 \mathrm{E}-01$ \\
\hline $2.00 \mathrm{E}+00$ & $9.52 \mathrm{E}+01$ & $1.50 \mathrm{E}+20$ & 1.79E-01 & $1.38 \mathrm{E}+20$ & $1.65 \mathrm{E}-01$ & $1.16 \mathrm{E}+20$ & $1.38 \mathrm{E}-01$ \\
\hline $2.30 \mathrm{E}+00$ & $9.38 \mathrm{E}+01$ & $1.90 \mathrm{E}+20$ & $2.22 \mathrm{E}-01$ & $1.65 \mathrm{E}+20$ & $1.94 \mathrm{E}-01$ & $1.51 \mathrm{E}+20$ & 1.77E-01 \\
\hline $2.60 \mathrm{E}+00$ & $1.12 \mathrm{E}+02$ & $1.75 \mathrm{E}+20$ & $2.45 \mathrm{E}-01$ & $1.55 \mathrm{E}+20$ & $2.16 \mathrm{E}-01$ & $1.42 \mathrm{E}+20$ & $1.99 \mathrm{E}-01$ \\
\hline $2.90 \mathrm{E}+00$ & $1.24 \mathrm{E}+02$ & $1.32 \mathrm{E}+20$ & 2.03E-01 & $1.16 \mathrm{E}+20$ & $1.79 \mathrm{E}-01$ & $1.02 \mathrm{E}+20$ & $1.58 \mathrm{E}-01$ \\
\hline $3.30 \mathrm{E}+00$ & $1.33 \mathrm{E}+02$ & $1.25 \mathrm{E}+20$ & 2.09E-01 & $1.16 \mathrm{E}+20$ & $1.93 \mathrm{E}-01$ & $1.07 \mathrm{E}+20$ & $1.78 \mathrm{E}-01$ \\
\hline $3.70 \mathrm{E}+00$ & $1.35 \mathrm{E}+02$ & $8.66 \mathrm{E}+19$ & $1.46 \mathrm{E}-01$ & $7.69 E+19$ & $1.30 \mathrm{E}-01$ & $7.44 \mathrm{E}+19$ & $1.26 \mathrm{E}-01$ \\
\hline $4.10 \mathrm{E}+00$ & $1.50 \mathrm{E}+02$ & $6.90 \mathrm{E}+19$ & $1.29 \mathrm{E}-01$ & $5.91 \mathrm{E}+19$ & $1.11 \mathrm{E}-01$ & $5.53 \mathrm{E}+19$ & $1.03 \mathrm{E}-01$ \\
\hline $4.50 \mathrm{E}+00$ & $1.58 \mathrm{E}+02$ & $5.30 \mathrm{E}+19$ & $1.05 \mathrm{E}-01$ & $4.33 E+19$ & 8.57E-02 & $4.40 \mathrm{E}+19$ & 8.71E-02 \\
\hline $5.00 \mathrm{E}+00$ & $1.69 \mathrm{E}+02$ & $4.98 \mathrm{E}+19$ & $1.05 \mathrm{E}-01$ & $4.69 \mathrm{E}+19$ & $9.89 \mathrm{E}-02$ & $3.97 \mathrm{E}+19$ & 8.37E-02 \\
\hline $5.50 \mathrm{E}+00$ & $1.76 \mathrm{E}+02$ & $3.16 E+19$ & $6.96 \mathrm{E}-02$ & $3.02 E+19$ & $6.66 \mathrm{E}-02$ & $2.82 \mathrm{E}+19$ & $6.22 \mathrm{E}-02$ \\
\hline
\end{tabular}




\begin{tabular}{|c|c|c|c|c|c|c|c|}
\hline & DPA xsec & \multicolumn{2}{|c|}{$\mathbf{2 - 3}$} & \multicolumn{2}{c|}{$\mathbf{2 - 2}$} & \multicolumn{2}{c|}{$\mathbf{2 - 1}$} \\
\hline $\begin{array}{c}\text { Upper } \\
\text { Energy(MeV) }\end{array}$ & keV-barn & Total Fluence & DPA & Total Fluence & DPA & Total Fluence & DPA \\
\hline $6.00 \mathrm{E}+00$ & $1.83 \mathrm{E}+02$ & $2.23 \mathrm{E}+19$ & $5.11 \mathrm{E}-02$ & $2.14 \mathrm{E}+19$ & $4.90 \mathrm{E}-02$ & $1.80 \mathrm{E}+19$ & $4.12 \mathrm{E}-02$ \\
\hline $6.70 \mathrm{E}+00$ & $1.89 \mathrm{E}+02$ & $2.18 \mathrm{E}+19$ & $5.15 \mathrm{E}-02$ & $1.86 \mathrm{E}+19$ & $4.40 \mathrm{E}-02$ & $1.88 \mathrm{E}+19$ & $4.46 \mathrm{E}-02$ \\
\hline $7.40 \mathrm{E}+00$ & $1.97 \mathrm{E}+02$ & $1.21 \mathrm{E}+19$ & $2.97 \mathrm{E}-02$ & $1.05 \mathrm{E}+19$ & $2.59 \mathrm{E}-02$ & $1.03 \mathrm{E}+19$ & $2.54 \mathrm{E}-02$ \\
\hline $8.20 \mathrm{E}+00$ & $2.03 \mathrm{E}+02$ & $7.18 \mathrm{E}+18$ & $1.82 \mathrm{E}-02$ & $7.40 \mathrm{E}+18$ & $1.88 \mathrm{E}-02$ & $7.28 \mathrm{E}+18$ & $1.85 \mathrm{E}-02$ \\
\hline $9.00 \mathrm{E}+00$ & $2.15 \mathrm{E}+02$ & $3.89 \mathrm{E}+18$ & $1.04 \mathrm{E}-02$ & $3.28 \mathrm{E}+18$ & $8.80 \mathrm{E}-03$ & $3.50 \mathrm{E}+18$ & $9.38 \mathrm{E}-03$ \\
\hline $1.00 \mathrm{E}+01$ & $2.26 \mathrm{E}+02$ & $3.02 \mathrm{E}+18$ & $8.51 \mathrm{E}-03$ & $2.44 \mathrm{E}+18$ & $6.89 \mathrm{E}-03$ & $2.50 \mathrm{E}+18$ & $7.05 \mathrm{E}-03$ \\
\hline $1.10 \mathrm{E}+01$ & $2.38 \mathrm{E}+02$ & $1.13 \mathrm{E}+18$ & $3.36 \mathrm{E}-03$ & $1.27 \mathrm{E}+18$ & $3.77 \mathrm{E}-03$ & $9.30 \mathrm{E}+17$ & $2.76 \mathrm{E}-03$ \\
\hline $1.20 \mathrm{E}+01$ & $2.47 \mathrm{E}+02$ & $5.75 \mathrm{E}+17$ & $1.78 \mathrm{E}-03$ & $2.90 \mathrm{E}+17$ & $8.97 \mathrm{E}-04$ & $2.50 \mathrm{E}+17$ & $7.74 \mathrm{E}-04$ \\
\hline $1.30 \mathrm{E}+01$ & $2.59 \mathrm{E}+02$ & $2.59 \mathrm{E}+17$ & $8.36 \mathrm{E}-04$ & $2.07 \mathrm{E}+17$ & $6.70 \mathrm{E}-04$ & $3.65 \mathrm{E}+17$ & $1.18 \mathrm{E}-03$ \\
\hline $1.40 \mathrm{E}+01$ & $2.71 \mathrm{E}+02$ & $7.73 \mathrm{E}+16$ & $2.62 \mathrm{E}-04$ & $2.80 \mathrm{E}+17$ & $9.49 \mathrm{E}-04$ & $1.43 \mathrm{E}+17$ & $4.86 \mathrm{E}-04$ \\
\hline $1.50 \mathrm{E}+01$ & $2.90 \mathrm{E}+02$ & $9.59 \mathrm{E}+16$ & $3.48 \mathrm{E}-04$ & $2.50 \mathrm{E}+17$ & $9.07 \mathrm{E}-04$ & $4.31 \mathrm{E}+16$ & $1.56 \mathrm{E}-04$ \\
\hline $1.60 \mathrm{E}+01$ & $2.93 \mathrm{E}+02$ & $3.80 \mathrm{E}+16$ & $1.39 \mathrm{E}-04$ & $0.00 \mathrm{E}+00$ & $0.00 \mathrm{E}+00$ & $0.00 \mathrm{E}+00$ & $0.00 \mathrm{E}+00$ \\
\hline $1.70 \mathrm{E}+01$ & $2.93 \mathrm{E}+02$ & $0.00 \mathrm{E}+00$ & $0.00 \mathrm{E}+00$ & $0.00 \mathrm{E}+00$ & $0.00 \mathrm{E}+00$ & $0.00 \mathrm{E}+00$ & $0.00 \mathrm{E}+00$ \\
\hline $1.80 \mathrm{E}+01$ & $2.98 \mathrm{E}+02$ & $0.00 \mathrm{E}+00$ & $0.00 \mathrm{E}+00$ & $0.00 \mathrm{E}+00$ & $0.00 \mathrm{E}+00$ & $0.00 \mathrm{E}+00$ & $0.00 \mathrm{E}+00$ \\
\hline $1.90 \mathrm{E}+01$ & $3.07 \mathrm{E}+02$ & $0.00 \mathrm{E}+00$ & $0.00 \mathrm{E}+00$ & $0.00 \mathrm{E}+00$ & $0.00 \mathrm{E}+00$ & $0.00 \mathrm{E}+00$ & $0.00 \mathrm{E}+00$ \\
\hline $2.00 \mathrm{E}+01$ & $3.16 \mathrm{E}+02$ & $0.00 \mathrm{E}+00$ & $0.00 \mathrm{E}+00$ & $0.00 \mathrm{E}+00$ & $0.00 \mathrm{E}+00$ & $0.00 \mathrm{E}+00$ & $0.00 \mathrm{E}+00$ \\
\hline & & & $3.47 \mathrm{E}+00$ & & $3.10 \mathrm{E}+00$ & & $\mathbf{2 . 8 8 E}+00$ \\
\hline
\end{tabular}


Table 21. Calculated fluence and DPA for capsule 1 of the UCSB experiment.

\begin{tabular}{|c|c|c|c|}
\hline & DPA xsec & \multicolumn{2}{|c|}{$1-1$} \\
\hline $\begin{array}{c}\text { Upper } \\
\text { Energy(MeV) }\end{array}$ & keV-barn & Total Fluence & DPA \\
\hline $1.00 \mathrm{E}-10$ & 0 & $0.00 \mathrm{E}+00$ & \\
\hline $1.00 \mathrm{E}-09$ & 7.73 & $2.09 \mathrm{E}+17$ & $2.02 \mathrm{E}-05$ \\
\hline $1.00 \mathrm{E}-08$ & 2.444 & $3.03 \mathrm{E}+19$ & $9.26 \mathrm{E}-04$ \\
\hline $2.30 \mathrm{E}-08$ & 1.278 & $1.14 \mathrm{E}+20$ & $1.82 \mathrm{E}-03$ \\
\hline $5.00 \mathrm{E}-08$ & 0.857 & $2.72 \mathrm{E}+20$ & $2.92 \mathrm{E}-03$ \\
\hline $7.60 \mathrm{E}-08$ & 0.644 & $2.00 \mathrm{E}+20$ & $1.61 \mathrm{E}-03$ \\
\hline $1.15 \mathrm{E}-07$ & 0.523 & $1.74 \mathrm{E}+20$ & $1.14 \mathrm{E}-03$ \\
\hline $1.70 \mathrm{E}-07$ & 0.428 & $1.14 \mathrm{E}+20$ & $6.12 \mathrm{E}-04$ \\
\hline $2.55 \mathrm{E}-07$ & 0.351 & $8.40 \mathrm{E}+19$ & $3.69 \mathrm{E}-04$ \\
\hline $3.80 \mathrm{E}-07$ & 0.287 & $7.35 \mathrm{E}+19$ & $2.64 \mathrm{E}-04$ \\
\hline $5.50 \mathrm{E}-07$ & 0.237 & $6.73 \mathrm{E}+19$ & $1.99 \mathrm{E}-04$ \\
\hline $8.40 \mathrm{E}-07$ & 0.194 & $7.43 \mathrm{E}+19$ & $1.80 \mathrm{E}-04$ \\
\hline $1.28 \mathrm{E}-06$ & 0.157 & $7.37 \mathrm{E}+19$ & $1.45 \mathrm{E}-04$ \\
\hline $1.90 \mathrm{E}-06$ & 0.128 & $6.85 \mathrm{E}+19$ & $1.10 \mathrm{E}-04$ \\
\hline $2.80 \mathrm{E}-06$ & 0.105 & $6.83 \mathrm{E}+19$ & $8.96 \mathrm{E}-05$ \\
\hline $4.25 \mathrm{E}-06$ & 0.086 & $7.51 \mathrm{E}+19$ & $8.07 \mathrm{E}-05$ \\
\hline $6.30 \mathrm{E}-06$ & 0.07 & $7.02 \mathrm{E}+19$ & $6.14 \mathrm{E}-05$ \\
\hline $9.20 \mathrm{E}-06$ & 0.058 & $6.57 \mathrm{E}+19$ & $4.77 \mathrm{E}-05$ \\
\hline $1.35 \mathrm{E}-05$ & 0.048 & $6.87 \mathrm{E}+19$ & $4.12 \mathrm{E}-05$ \\
\hline $2.10 \mathrm{E}-05$ & 0.038 & $8.16 \mathrm{E}+19$ & $3.88 \mathrm{E}-05$ \\
\hline $3.00 \mathrm{E}-05$ & 0.031 & $6.68 \mathrm{E}+19$ & $2.59 \mathrm{E}-05$ \\
\hline $4.50 \mathrm{E}-05$ & 0.026 & $7.65 \mathrm{E}+19$ & $2.49 \mathrm{E}-05$ \\
\hline $6.90 \mathrm{E}-05$ & 0.021 & $8.10 \mathrm{E}+19$ & $2.13 \mathrm{E}-05$ \\
\hline $1.00 \mathrm{E}-04$ & 0.017 & $7.30 \mathrm{E}+19$ & $1.55 \mathrm{E}-05$ \\
\hline $1.35 \mathrm{E}-04$ & 0.014 & $5.94 \mathrm{E}+19$ & $1.04 \mathrm{E}-05$ \\
\hline $1.70 \mathrm{E}-04$ & 0.014 & $4.89 \mathrm{E}+19$ & $8.55 \mathrm{E}-06$ \\
\hline $2.20 \mathrm{E}-04$ & 0.011 & $5.26 \mathrm{E}+19$ & $7.24 \mathrm{E}-06$ \\
\hline
\end{tabular}




\begin{tabular}{|c|c|c|c|}
\hline $2.80 \mathrm{E}-04$ & 0.011 & $5.24 \mathrm{E}+19$ & $7.20 \mathrm{E}-06$ \\
\hline $3.60 \mathrm{E}-04$ & 0.01 & $5.11 \mathrm{E}+19$ & $6.39 \mathrm{E}-06$ \\
\hline $4.50 \mathrm{E}-04$ & 0.007 & $4.24 \mathrm{E}+19$ & $3.71 \mathrm{E}-06$ \\
\hline $5.75 \mathrm{E}-04$ & 0.005 & $5.07 \mathrm{E}+19$ & $3.17 \mathrm{E}-06$ \\
\hline $7.60 \mathrm{E}-04$ & 0.129 & $5.57 \mathrm{E}+19$ & $8.98 \mathrm{E}-05$ \\
\hline $9.60 \mathrm{E}-04$ & 0.308 & $4.82 \mathrm{E}+19$ & $1.86 \mathrm{E}-04$ \\
\hline $1.28 \mathrm{E}-03$ & 0.672 & $5.79 \mathrm{E}+19$ & $4.86 \mathrm{E}-04$ \\
\hline $1.60 \mathrm{E}-03$ & 0.477 & $4.57 \mathrm{E}+19$ & $2.72 \mathrm{E}-04$ \\
\hline $2.00 \mathrm{E}-03$ & 0.511 & $4.42 \mathrm{E}+19$ & $2.82 \mathrm{E}-04$ \\
\hline $2.70 \mathrm{E}-03$ & 0.568 & $6.01 \mathrm{E}+19$ & $4.26 \mathrm{E}-04$ \\
\hline $3.40 \mathrm{E}-03$ & 0.632 & $4.92 \mathrm{E}+19$ & $3.89 \mathrm{E}-04$ \\
\hline $4.50 \mathrm{E}-03$ & 0.822 & $5.83 \mathrm{E}+19$ & $5.99 \mathrm{E}-04$ \\
\hline $5.50 \mathrm{E}-03$ & 0.826 & $4.42 \mathrm{E}+19$ & $4.57 \mathrm{E}-04$ \\
\hline $7.20 \mathrm{E}-03$ & 1.671 & $6.01 \mathrm{E}+19$ & $1.26 \mathrm{E}-03$ \\
\hline $9.20 \mathrm{E}-03$ & 3.709 & $4.94 \mathrm{E}+19$ & $2.29 \mathrm{E}-03$ \\
\hline $1.20 \mathrm{E}-02$ & 1.491 & $5.89 \mathrm{E}+19$ & $1.10 \mathrm{E}-03$ \\
\hline $1.50 \mathrm{E}-02$ & 1.156 & $5.16 \mathrm{E}+19$ & $7.46 \mathrm{E}-04$ \\
\hline $1.90 \mathrm{E}-02$ & 0.973 & $5.16 \mathrm{E}+19$ & $6.28 \mathrm{E}-04$ \\
\hline $2.55 \mathrm{E}-02$ & 0.555 & $7.60 \mathrm{E}+19$ & $5.27 \mathrm{E}-04$ \\
\hline $3.20 \mathrm{E}-02$ & 26.762 & $6.67 \mathrm{E}+19$ & $2.23 \mathrm{E}-02$ \\
\hline $4.00 \mathrm{E}-02$ & 8.161 & $3.09 \mathrm{E}+19$ & $3.15 \mathrm{E}-03$ \\
\hline $5.25 \mathrm{E}-02$ & 6.515 & $6.91 \mathrm{E}+19$ & $5.63 \mathrm{E}-03$ \\
\hline $6.60 \mathrm{E}-02$ & 6.576 & $6.75 \mathrm{E}+19$ & $5.55 \mathrm{E}-03$ \\
\hline $8.80 \mathrm{E}-02$ & 13.137 & $9.78 \mathrm{E}+19$ & $1.61 \mathrm{E}-02$ \\
\hline $1.10 \mathrm{E}-01$ & 11.84 & $5.89 \mathrm{E}+19$ & $8.72 \mathrm{E}-03$ \\
\hline $1.35 \mathrm{E}-01$ & 11.27 & $9.09 \mathrm{E}+19$ & $1.28 \mathrm{E}-02$ \\
\hline $1.60 \mathrm{E}-01$ & 21.53 & $5.22 \mathrm{E}+19$ & $1.40 \mathrm{E}-02$ \\
\hline $1.90 \mathrm{E}-01$ & 15.85 & $7.05 \mathrm{E}+19$ & $1.40 \mathrm{E}-02$ \\
\hline $2.20 \mathrm{E}-01$ & 22.01 & $5.77 \mathrm{E}+19$ & $1.59 \mathrm{E}-02$ \\
\hline $2.55 \mathrm{E}-01$ & 18.27 & $7.04 \mathrm{E}+19$ & $1.61 \mathrm{E}-02$ \\
\hline $2.90 \mathrm{E}-01$ & 17.37 & $7.70 \mathrm{E}+19$ & $1.67 \mathrm{E}-02$ \\
\hline $3.20 \mathrm{E}-01$ & 13.51 & $5.46 \mathrm{E}+19$ & $9.22 \mathrm{E}-03$ \\
\hline
\end{tabular}




\begin{tabular}{|c|c|c|c|}
\hline $3.60 \mathrm{E}-01$ & 25.14 & $7.33 \mathrm{E}+19$ & $2.30 \mathrm{E}-02$ \\
\hline $4.00 \mathrm{E}-01$ & 46.52 & $6.61 \mathrm{E}+19$ & $3.84 \mathrm{E}-02$ \\
\hline $4.50 \mathrm{E}-01$ & 43.04 & $6.33 \mathrm{E}+19$ & $3.41 \mathrm{E}-02$ \\
\hline $5.00 \mathrm{E}-01$ & 36.4 & $7.03 \mathrm{E}+19$ & $3.20 \mathrm{E}-02$ \\
\hline $5.50 \mathrm{E}-01$ & 33.22 & $6.63 \mathrm{E}+19$ & $2.75 \mathrm{E}-02$ \\
\hline $6.00 \mathrm{E}-01$ & 34.18 & $6.39 \mathrm{E}+19$ & $2.73 \mathrm{E}-02$ \\
\hline $6.60 \mathrm{E}-01$ & 19.64 & $7.89 \mathrm{E}+19$ & $1.94 \mathrm{E}-02$ \\
\hline $7.20 \mathrm{E}-01$ & 48.89 & $8.42 \mathrm{E}+19$ & $5.15 \mathrm{E}-02$ \\
\hline $7.80 \mathrm{E}-01$ & 74.22 & $7.64 \mathrm{E}+19$ & $7.09 \mathrm{E}-02$ \\
\hline $8.40 \mathrm{E}-01$ & 44.02 & $6.10 \mathrm{E}+19$ & $3.36 \mathrm{E}-02$ \\
\hline $9.20 \mathrm{E}-01$ & 40.97 & $7.36 \mathrm{E}+19$ & $3.77 \mathrm{E}-02$ \\
\hline $1.00 \mathrm{E}+00$ & 50.95 & $6.70 \mathrm{E}+19$ & $4.27 \mathrm{E}-02$ \\
\hline $1.20 \mathrm{E}+00$ & 50.09 & $1.52 \mathrm{E}+20$ & $9.53 \mathrm{E}-02$ \\
\hline $1.40 \mathrm{E}+00$ & 64.54 & $1.37 \mathrm{E}+20$ & $1.11 \mathrm{E}-01$ \\
\hline $1.60 \mathrm{E}+00$ & 73.58 & $1.15 \mathrm{E}+20$ & $1.06 \mathrm{E}-01$ \\
\hline $1.80 \mathrm{E}+00$ & 76.46 & $1.06 \mathrm{E}+20$ & $1.01 \mathrm{E}-01$ \\
\hline $2.00 \mathrm{E}+00$ & 95.15 & $9.01 \mathrm{E}+19$ & $1.07 \mathrm{E}-01$ \\
\hline $2.30 \mathrm{E}+00$ & 93.75 & $1.14 \mathrm{E}+20$ & $1.34 \mathrm{E}-01$ \\
\hline $2.60 \mathrm{E}+00$ & 112.05 & $1.09 \mathrm{E}+20$ & $1.53 \mathrm{E}-01$ \\
\hline $2.90 \mathrm{E}+00$ & 123.55 & $7.97 \mathrm{E}+19$ & $1.23 \mathrm{E}-01$ \\
\hline $3.30 \mathrm{E}+00$ & 133.45 & $8.08 \mathrm{E}+19$ & $1.35 \mathrm{E}-01$ \\
\hline $3.70 \mathrm{E}+00$ & 135.25 & $5.44 \mathrm{E}+19$ & $9.20 \mathrm{E}-02$ \\
\hline $4.10 \mathrm{E}+00$ & 149.55 & $4.18 \mathrm{E}+19$ & $7.81 \mathrm{E}-02$ \\
\hline $4.50 \mathrm{E}+00$ & 158.25 & $3.26 \mathrm{E}+19$ & $6.44 \mathrm{E}-02$ \\
\hline $5.00 \mathrm{E}+00$ & 168.55 & $3.05 \mathrm{E}+19$ & $6.43 \mathrm{E}-02$ \\
\hline $5.50 \mathrm{E}+00$ & 176.46 & $1.97 \mathrm{E}+19$ & $4.34 \mathrm{E}-02$ \\
\hline $6.00 \mathrm{E}+00$ & 183.06 & $1.53 \mathrm{E}+19$ & $3.50 \mathrm{E}-02$ \\
\hline $6.70 \mathrm{E}+00$ & 189.26 & $1.25 \mathrm{E}+19$ & $2.95 \mathrm{E}-02$ \\
\hline $7.40 \mathrm{E}+00$ & 196.67 & $7.38 \mathrm{E}+18$ & $1.82 \mathrm{E}-02$ \\
\hline $8.20 \mathrm{E}+00$ & 203.37 & $4.25 \mathrm{E}+18$ & $1.08 \mathrm{E}-02$ \\
\hline $9.00 \mathrm{E}+00$ & 214.58 & $2.74 \mathrm{E}+18$ & $7.36 \mathrm{E}-03$ \\
\hline $1.00 \mathrm{E}+01$ & 225.69 & $1.57 \mathrm{E}+18$ & $4.44 \mathrm{E}-03$ \\
\hline
\end{tabular}




\begin{tabular}{|c|c|c|c|}
$1.10 \mathrm{E}+01$ & 237.6 & $4.57 \mathrm{E}+17$ & $1.36 \mathrm{E}-03$ \\
\hline $1.20 \mathrm{E}+01$ & 247.41 & $4.60 \mathrm{E}+17$ & $1.42 \mathrm{E}-03$ \\
\hline $1.30 \mathrm{E}+01$ & 258.52 & $2.11 \mathrm{E}+17$ & $6.82 \mathrm{E}-04$ \\
\hline $1.40 \mathrm{E}+01$ & 271.35 & $1.20 \mathrm{E}+17$ & $4.08 \mathrm{E}-04$ \\
\hline $1.50 \mathrm{E}+01$ & 290.27 & $5.66 \mathrm{E}+16$ & $2.05 \mathrm{E}-04$ \\
\hline $1.60 \mathrm{E}+01$ & 293.2 & $0.00 \mathrm{E}+00$ & $0.00 \mathrm{E}+00$ \\
\hline $1.70 \mathrm{E}+01$ & 292.73 & $0.00 \mathrm{E}+00$ & $0.00 \mathrm{E}+00$ \\
\hline $1.80 \mathrm{E}+01$ & 297.65 & $0.00 \mathrm{E}+00$ & $0.00 \mathrm{E}+00$ \\
\hline $1.90 \mathrm{E}+01$ & 307.26 & $0.00 \mathrm{E}+00$ & $0.00 \mathrm{E}+00$ \\
\hline $2.00 \mathrm{E}+01$ & 316.36 & $0.00 \mathrm{E}+00$ & $0.00 \mathrm{E}+00$ \\
\hline & & & $2.14 \mathrm{E}+00$ \\
\hline
\end{tabular}




\section{References}

[1] PLN-2996, "Irradiation Test Plan for the ATR National Scientific User Facility University of California Santa Barbara," Rev. 3, July 27, 2009.

[2] Tim Goorley, Jeffrey S. Bull, Forrest B. Brown, et. al., "Release of MCNP5_RSICC_1.30," Trans. Am. Nucl. Soc., Vol. 91, pp. 693-694 (2004).

[3] X-5 Monte Carlo Team, "MCNP_A General Monte Carlo N-Particle Transport Code, Version 5," Volume I, LA-UR-03-1987, Los Alamos National Laboratory, April 24, 2003 (Revised 6/30/2004) and Volume II, LA-CP-0245, Los Alamos National Laboratory, April 24, 2003 (Revised 6/30/2004) (Vol. II is available with a licensed copy of MCNP).

[4] E. T. Boulette, W. L. Bunch, "Analysis of ZPPR/FTR Shield Experiments Gamma Distributions," WHAN-FR-13, February 1971.

[5] M. A. Lillo, J. R. Mitchell, "Software Management, MCNP Version 5, Release 1.40," INL/INT-08-15171 Rev. 0, August 2010, INL Record Number 234166.

[6] "MCNP5 Version 1.60 Software Management Plan," PLN-4099, Revision 0, January 2012

[7] S. S. Kim, B. G. Schnitzler, "Advanced Test Reactor: Serpentine Arrangement of Highly Enriched Water-Moderated Uranium-Aluminide Fuel Plates Reflected by Beryllium," INL/EXT-05-00780, September 2005; published in "International Handbook of Evaluated Criticality Safety Benchmark Experiments," NEA/NSC/DOC/(95)03/II, Volume II, HEUMET-THERM-022, September 2005 Edition. 
Appendix A: DPA Cross-Sections 


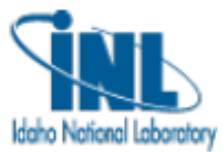

\section{Re: PNNL External Website Inquiry}

3 messages

G. Robert Odette <odette@engineering.ucsb.edu>

Sun, Jun 23,2013 at 12:47 PM

To: Joseph W Nielsen <Joseph.Nielsen@inl.gov>

Hi Joe,

Below is an exchange with Larry Greenwood at PNNL. Larry developed the SPECTER code many years ago for damage calculations including dpa. He suggests the 100 group energy structure shown below. I could ask him for the corresponding dpa cross sections he uses if you like. BTW I am not retiring from research, only classroom teaching. Thanks for your help on this.

Regards,

Bob

On 6/23/13 11:25 AM, Greenwood, Larry R wrote:

Bob - Thanks for your comments - I completely agree with your comments! I have attached the energy grid below. $\mathrm{I}$ can also send it in other formats if more convenient.

One minor point is that the ASTM standard E693 has 640 energy groups. My version in SPECTER has only 100 groups which I have found to be adequate.

I also hope our paths cross again in the near future! Good luck on your retirement! I am about a year behind you it appears. I may hang on here a few more years, perhaps going part time at some point.

Best regards,

Larry

101

1.0000E-10 1.0000E-09 1.0000E-08 2.3000E-08 5.0000E-08 7.6000E-08 1.1500E-07 1.7000E-07 2.5500E-07 3.8000E-07 5.5000E-07 8.4000E-07 1.2750E-06 1.9000E-06 2.8000E-06 4.2500E-06 6.3000E-06 9.2000E-06 1.3500E-05 2.1000E-05 3.0000E-05 4.5000E-05 6.9000E-05 1.0000E-04 1.3500E-04 1.7000E-04 2.2000E-04 2.8000E-04 3.6000E-04 4.5000E-04 5.7500E-04 7.6000E-04 9.6000E-04 1.2750E-03 1.6000E-03 2.0000E-03 2.7000E-03 3.4000E-03 4.5000E-03 5.5000E-03 7.2000E-03 9.2000E-03 1.2000E-02 1.5000E-02 1.9000E-02 2.5500E-02 3.2000E-02 4.0000E-02 5.2500E-02 6.6000E-02 8.8000E-02 1.1000E-01 1.3500E-01 1.6000E-01 1.9000E-01 2.2000E-01 2.5500E-01 2.9000E-01 3.2000E-01 3.6000E-01 4.0000E-01 4.5000E-01 5.0000E-01 5.5000E-01 6.0000E-01 6.6000E-01 7.2000E-01 7.8000E-01 8.4000E-01 9.2000E-01 $1.0000 \mathrm{E}+001.2000 \mathrm{E}+001.4000 \mathrm{E}+001.6000 \mathrm{E}+001.8000 \mathrm{E}+002.0000 \mathrm{E}+002.3000 \mathrm{E}+00$ $2.6000 \mathrm{E}+002.9000 \mathrm{E}+003.3000 \mathrm{E}+003.7000 \mathrm{E}+00$ 4.1000E+00 4.5000E+00 5.0000E+00 5.5000E+00 6.0000E+00 6.7000E+00 7.4000E+00 8.2000E+00 9.0000E+00 1.0000E+01 1.1000E+01 1.2000E+01 1.3000E+01 1.4000E+01 1.5000E+01 1.6000E+01 1.7000E+01 $1.8000 \mathrm{E}+011.9000 \mathrm{E}+012.0000 \mathrm{E}+01$

Larry Greenwood, Pacific Northwest National Laboratory 
Laboratory Fellow

Phone: 509-375-5301

---Original Message-----

From: G. Robert Odette [mailto:odette@engineering.ucsb.edu]

Sent: Saturday, June 22, 2013 9:15 AM

To: Greenwood, Larry R

Subject: Re: PNNL External Website Inquiry

Hi Larry,

Thanks so much for getting right back to me! It would make sense to adapt your 100 group structure since it is tailored to damage calculations. This is also the case since your dpa cross section is the ASTM standard. Would it be convenient for you to send me the group structure in a simple file or even in an e-mail so I can pass it on the the analyst at INL. Regarding you other comments, see below. Thanks again.

Regards,

Bob

PS It would be great if our paths crossed at some point in the near future! I am retiring (I will be 70 ) from teaching after the fall quarter, but will continue to run our very active research group as a Research Prof.

On 6/21/13 8:30 AM, Greenwood, Larry R wrote:

Hi Bob,

It is good to hear from you and I hope you and family are doing well!

I am still using the SPECTER computer code based on your DISCS program and that code has a standard 100 group energy grid (also a 100 group recoil energy grid so 10,000 group array for every element. The SPECTER computer code is available on the IAEA web site if you don't already have it. Due to the large effort spent in creating all the libraries for SPECTER, obviously we don't change the energy grid but tried to give sufficient detail with 100 groups to fit most applications.

\section{http://www-nds.iaea.org/irdf2002/codes/index.htmlx}

The IAEA has a CRP to look at possible updates to damage cross sections and models and I will be attending the first meeting in November. Many others are involved such as Roger Stoller to look at alternative functions to dpa. I am totally against alternate functions as a general dose unit to replace dpa. The overwhelming benefit of dpa is that is is at base a KERMA based DOSE UNIT!

SPECTER has not been updated for many years although the Fe dpa cross section is an ASTM standard. Whereas some are pushing for a complete update with current cross sections, there is a problem I think in doing this too frequently since it can become impossible to figure out which version of dpa cross sections are being used in publications and for correlation of data from many different irradiation sources.

Totally agreed.

The ASTM approach to the Fe standard provides some guidance on this. Codes such as NJOY can of course create dpa cross sections in any energy grid you like. However, putting together large libraries as used in SPECTER is still a formidable task that takes a lot of effort. There is also an effort to push dpa cross sections to much higher neutron energies. If you have any thoughts on any of this please let me know.

The need for higher energy cross sections is legitimate, and probably requires better nuclear reaction kinematics models. But I think a lot has been done on this already.

Larry Greenwood, Pacific Northwest National Laboratory Laboratory

Fellow

Phone: 509-375-5301 
From: odette@engineering.ucsb.edu [mailto:odette@engineering.ucsb.edu]

Sent: Friday, June 21, 2013 7:04 AM

To: Greenwood, Larry R

Subject: PNNL External Website Inquiry

PNNL External Website Inquiry

Name: Robert Odette

Sent: 6/21/2013 7:04:05 AM

Email: odette@engineering.ucsb.edu<mailto:odette@engineering.ucsb.edu>

Company: UCSB

Message

Hi Larry, Hope all is well. It has been a long time! I have a quick question. We had a NSUP irradiation in ATR and they will be doing as-run MPMC calculation of the dose and dose rate parameters. They ask about the group structure for dpa. Do you have a suggestion on a standard pick that is not too coarse or fine. Thanks. Regards, Bob

Thanks for the info. I will be able to get the cases ran this week and will have preliminary results next week. I spoke with Paul on the heat rates this morning and we are going to use what we currently have from the projection analysis and scale to the cycle averaged operating powers. I will pull the hourly data and see what the variation in lobe power was for the cycle, but since we were in A-10 near the SEFT, I don't expect much.

Joe

[Quoted text hidden]

\section{Verify This Message with Penango.p7s}

$$
7 \mathrm{~K}
$$

G. Robert Odette <odette@engineering.ucsb.edu>

To: Joseph W Nielsen <joseph.nielsen@inl.gov>

Mon, Jun 24, 2013 at 11:32 AM

Hi Joe,

Great!. I attach a 100 group file with the damage energy which can be converted to dpa by multiplying by 10 . If you would like this in a more convenient format let me know. The dpa should be for $\mathrm{Fe}$.

Regards,

Bob

On 6/24/13 9:24 AM, Joseph W Nielsen wrote: 


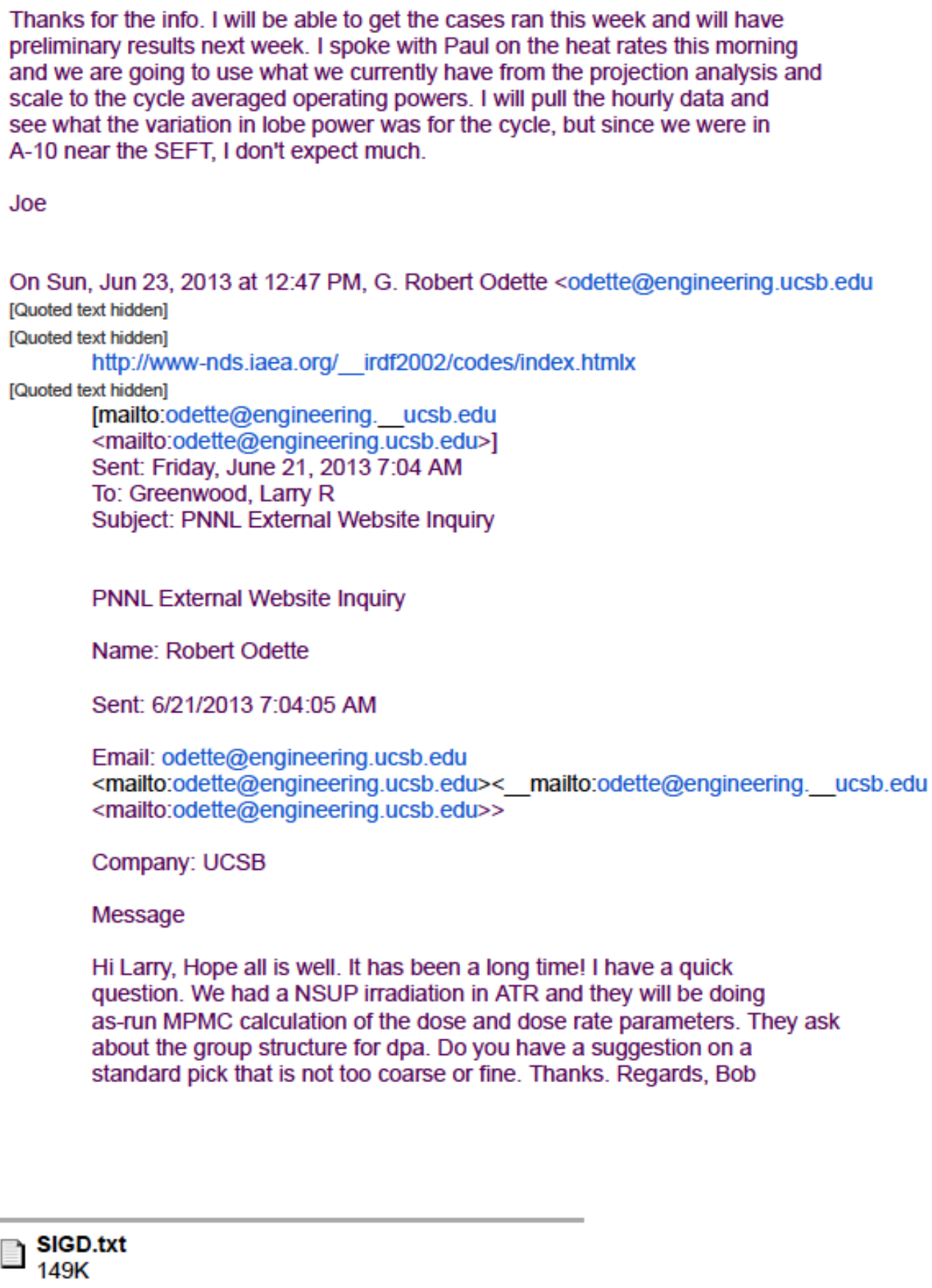


FILE SIGD from email above

\begin{tabular}{|c|c|c|c|}
\hline \\
\hline $\mathrm{EDL}=$ & $10.0 \mathrm{EV}$ & $\mathrm{TGAM}=528 \cdot \mathrm{EV}$ & \\
\hline LOWER & CROSS & LOWER & CROSS \\
\hline $\begin{array}{l}\text { ENERGY } \\
(\mathrm{MEV})\end{array}$ & $\begin{array}{c}\text { SECTION } \\
(\mathrm{KEV}-\mathrm{B})\end{array}$ & $\begin{array}{l}\text { ENERGY } \\
(\mathrm{MEV})\end{array}$ & $\begin{array}{c}\text { SECTION } \\
(\mathrm{KEV}-\mathrm{B})\end{array}$ \\
\hline 1. $000 \mathrm{E}-10$ & 1.340 & $8.80 E-02$ & 6.92 \\
\hline $1.000 \mathrm{E}-09$ & 0.424 & $1.10 \mathrm{E}-01$ & 6.52 \\
\hline $1.000 E-08$ & 0.222 & $1.35 E-01$ & 6.15 \\
\hline $2.300 E-08$ & 0.149 & $1.60 \mathrm{E}-01$ & 5.78 \\
\hline $5.000 \mathrm{E}-08$ & 0.112 & 1.90E-01 & 5.44 \\
\hline $7.600 E-08$ & 0.091 & $2.20 E-01$ & 5.13 \\
\hline $1.150 E-07$ & 0.074 & $2.55 \mathrm{E}-01$ & 4.83 \\
\hline $1.700 \mathrm{E}-07$ & 0.061 & $2.90 E-01$ & 4.62 \\
\hline $2.550 E-07$ & 0.050 & $3.20 E-01$ & 4.38 \\
\hline $3.800 E-07$ & 0.041 & $3.60 E-01$ & 4.16 \\
\hline $5.500 \mathrm{E}-07$ & 0.034 & $4.00 E-01$ & 3.94 \\
\hline $8.400 E-07$ & 0.027 & $4.50 E-01$ & 3.74 \\
\hline $1.275 E-06$ & 0.022 & $5.00 E-01$ & 3.56 \\
\hline $1.900 E-06$ & 0.018 & $5.50 E-01$ & 3.41 \\
\hline $2.800 E-06$ & 0.015 & $6.00 \mathrm{E}-01$ & 3.26 \\
\hline $4.250 E-06$ & 0.012 & $6.60 \mathrm{E}-01$ & 3.12 \\
\hline $6.300 E-06$ & 0.010 & $7.20 E-01$ & 2.98 \\
\hline $9.200 E-06$ & 0.008 & $7.80 \mathrm{E}-01$ & 2.89 \\
\hline $1.350 E-05$ & 0.007 & $8.40 E-01$ & 2.76 \\
\hline $2.100 E-05$ & 0.006 & $9.20 E-01$ & 2.64 \\
\hline $3.000 \mathrm{E}-05$ & 0.005 & $1.00 \mathrm{E}+00$ & 2.48 \\
\hline $4.500 E-05$ & 0.164 & 1.20E+00 & 2.28 \\
\hline $6.900 E-05$ & 0.521 & $1.40 E+00$ & 2.12 \\
\hline 1.000E-04 & 0.657 & $1.60 \mathrm{E}+00$ & 1.98 \\
\hline $1.350 E-04$ & 0.708 & $1.80 \mathrm{E}+00$ & 1.87 \\
\hline $1.700 \mathrm{E}-04$ & 0.790 & $2.00 \mathrm{E}+00$ & 1.75 \\
\hline $2.200 E-04$ & 0.910 & $2.30 \mathrm{E}+00$ & 1.63 \\
\hline $2.800 E-04$ & 1.068 & $2.60 E+00$ & 1.53 \\
\hline $3.600 \mathrm{E}-04$ & 1.250 & $2.90 \mathrm{E}+00$ & 1.43 \\
\hline $4.500 E-04$ & 1.485 & $3.30 \mathrm{E}+00$ & 1.34 \\
\hline $5.750 E-04$ & 1.785 & $3.70 E+00$ & 1.26 \\
\hline 7. $600 \mathrm{E}-04$ & 2.137 & $4.10 \mathrm{E}+00$ & 1.19 \\
\hline $9.600 \mathrm{E}-04$ & 2.539 & $4.50 \mathrm{E}+00$ & 1.12 \\
\hline $1.275 E-03$ & 2.980 & $5.00 \mathrm{E}+00$ & 1.06 \\
\hline $1.600 \mathrm{E}-03$ & 3.415 & $5.50 \mathrm{E}+00$ & 1.00 \\
\hline $2.000 E-03$ & 3.952 & $6.00 \mathrm{E}+00$ & 0.95 \\
\hline $2.700 \mathrm{E}-03$ & 4.515 & $6.70 \mathrm{E}+00$ & 0.89 \\
\hline $3.400 E-03$ & 5.072 & $7.40 \mathrm{E}+00$ & 0.84 \\
\hline $4.500 E-03$ & 5.586 & $8.20 E+00$ & 0.79 \\
\hline $5.500 \mathrm{E}-03$ & 6.082 & $9.00 \mathrm{E}+00$ & 0.74 \\
\hline $7.200 E-03$ & 6.577 & $1.00 \mathrm{E}+01$ & 0.70 \\
\hline $9.200 \mathrm{E}-03$ & 7.015 & 1.10E+01 & 0.67 \\
\hline 1.200E-02 & 7.371 & $1.20 \mathrm{E}+01$ & 0.64 \\
\hline $1.500 \mathrm{E}-02$ & 7.646 & 1.30E+01 & 0.61 \\
\hline 1.900E-02 & 7.872 & $1.40 \mathrm{E}+01$ & 0.59 \\
\hline $2.550 \mathrm{E}-02$ & 7.972 & $1.50 \mathrm{E}+01$ & 0.57 \\
\hline $3.200 E-02$ & 7.972 & $1.60 \mathrm{E}+01$ & 0.56 \\
\hline $4.000 E-02$ & 7.860 & $1.70 \mathrm{E}+01$ & 0.54 \\
\hline
\end{tabular}




$\begin{array}{llll}5.250 \mathrm{E}-02 & 7.648 & 1.80 \mathrm{E}+01 & 0.53 \\ 6.600 \mathrm{E}-02 & 7.323 & 1.90 \mathrm{E}+01 & 0.52 \\ & & 2.00 \mathrm{E}+01 & \end{array}$

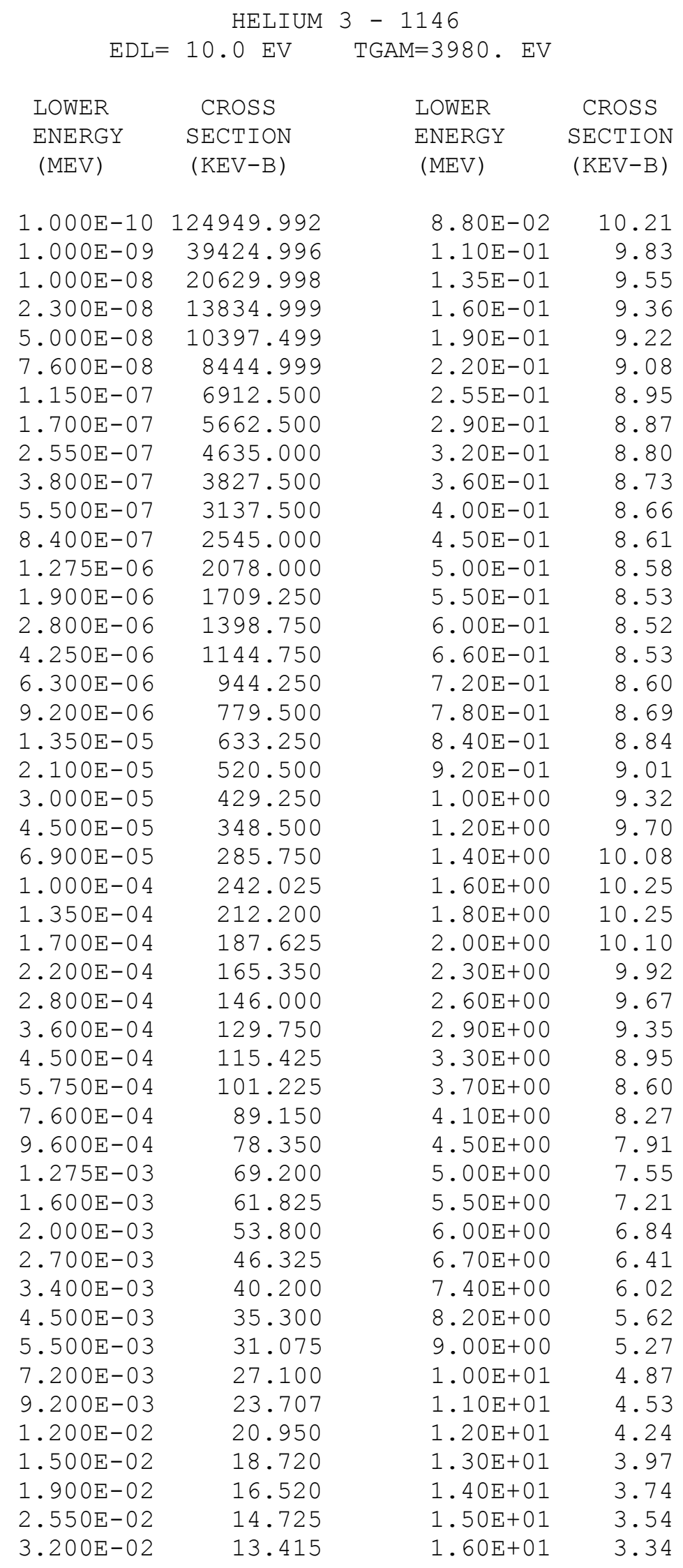




$\begin{array}{llll}4.000 \mathrm{E}-02 & 12.227 & 1.70 \mathrm{E}+01 & 3.15 \\ 5.250 \mathrm{E}-02 & 11.287 & 1.80 \mathrm{E}+01 & 3.00 \\ 6.600 \mathrm{E}-02 & 10.577 & 1.90 \mathrm{E}+01 & 2.87 \\ & & 2.00 \mathrm{E}+01 & \end{array}$

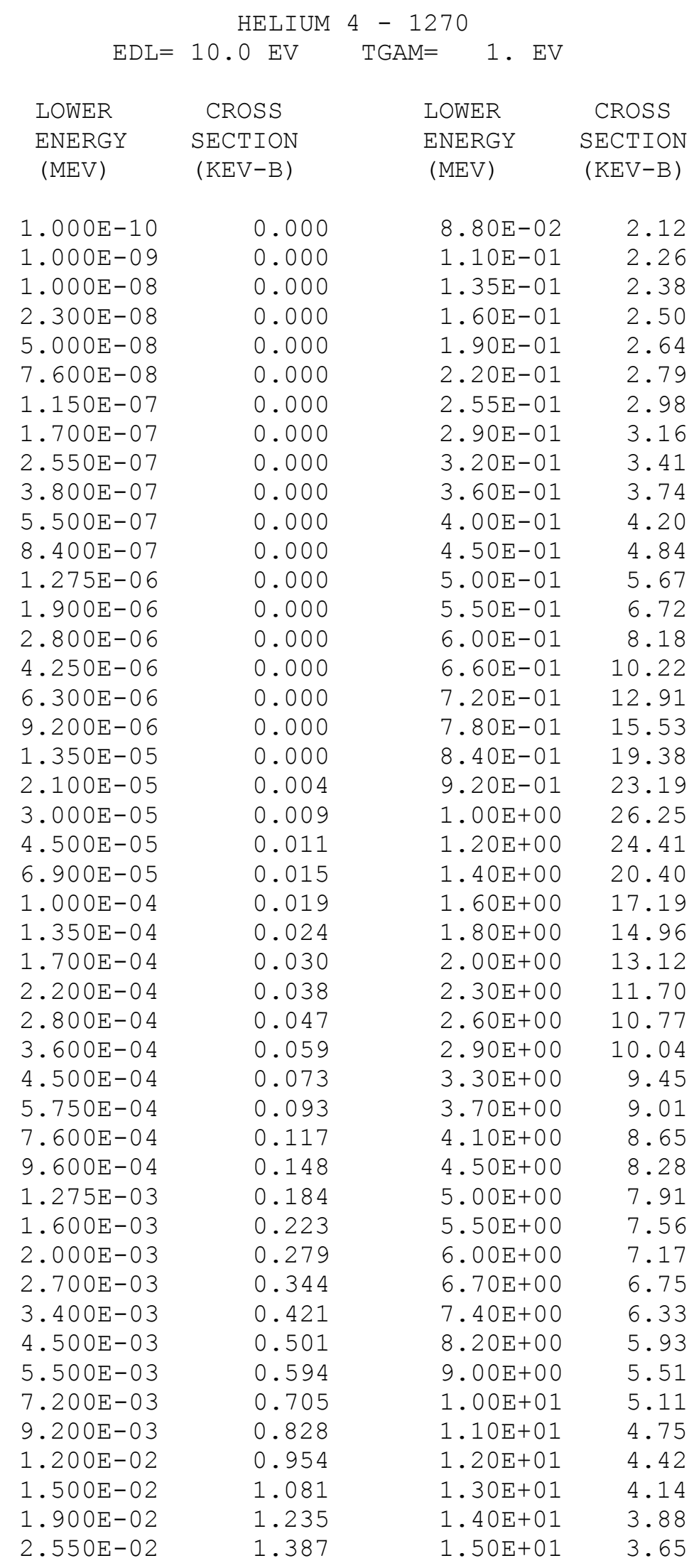




$\begin{array}{llll}3.200 \mathrm{E}-02 & 1.522 & 1.60 \mathrm{E}+01 & 3.44 \\ 4.000 \mathrm{E}-02 & 1.672 & 1.70 \mathrm{E}+01 & 3.26 \\ 5.250 \mathrm{E}-02 & 1.819 & 1.80 \mathrm{E}+01 & 3.10 \\ 6.600 \mathrm{E}-02 & 1.974 & 1.90 \mathrm{E}+01 & 2.95 \\ & & 2.00 \mathrm{E}+01 & \end{array}$

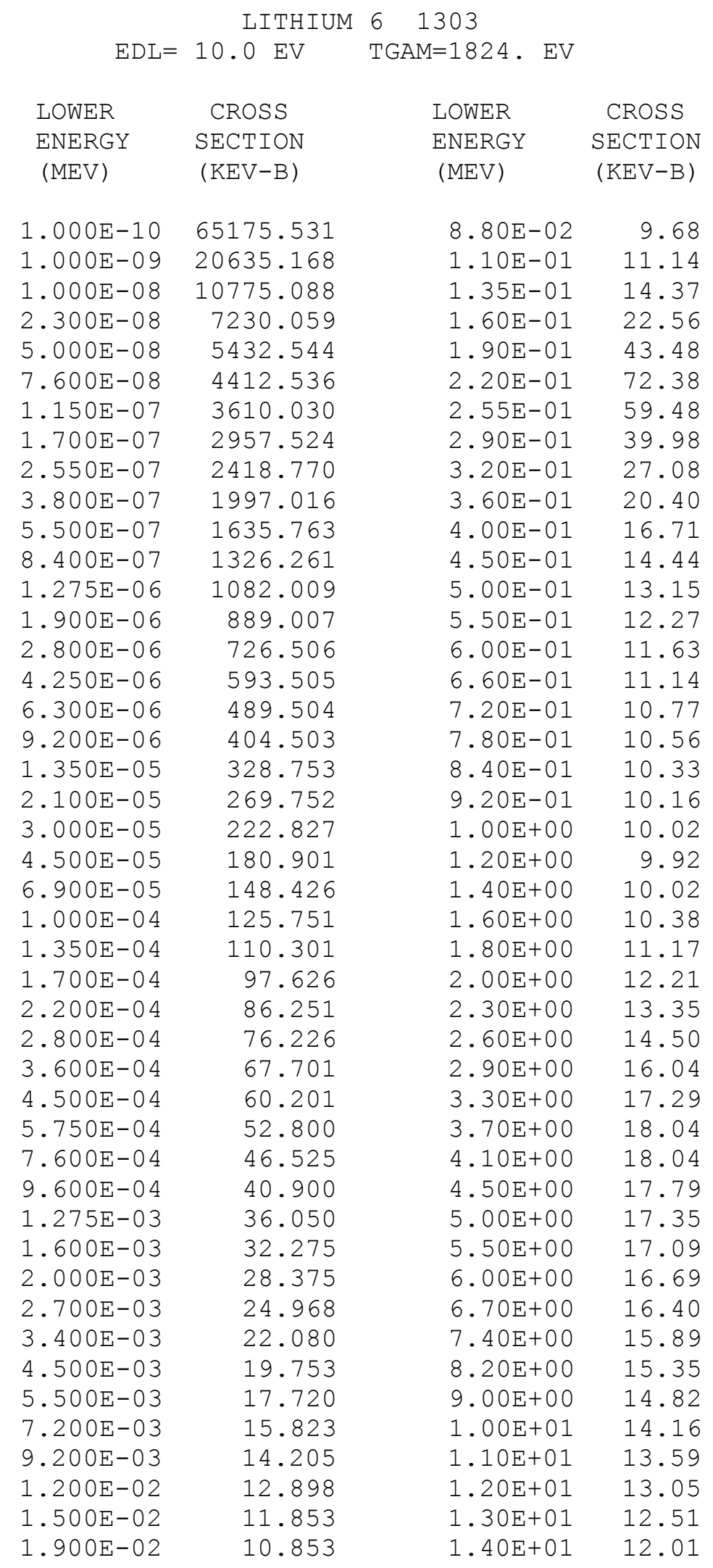




$\begin{array}{rrrr}2.550 \mathrm{E}-02 & 10.088 & 1.50 \mathrm{E}+01 & 11.57 \\ 3.200 \mathrm{E}-02 & 9.583 & 1.60 \mathrm{E}+01 & 11.14 \\ 4.000 \mathrm{E}-02 & 9.185 & 1.70 \mathrm{E}+01 & 10.75 \\ 5.250 \mathrm{E}-02 & 8.990 & 1.80 \mathrm{E}+01 & 10.42 \\ 6.600 \mathrm{E}-02 & 9.080 & 1.90 \mathrm{E}+01 & 10.13 \\ & & 2.00 \mathrm{E}+01 & \end{array}$

\begin{tabular}{|c|c|c|c|}
\hline $\mathrm{EDL}=$ & $\begin{array}{l}\text { LITHIUM } \\
10.0 \mathrm{EV}\end{array}$ & $\begin{array}{l}7 \quad 1272 \\
T G A M=7555 . \mathrm{EV}\end{array}$ & \\
\hline LOWER & CROSS & LOWER & CROSS \\
\hline $\begin{array}{l}\text { ENERGY } \\
(\mathrm{MEV})\end{array}$ & $\begin{array}{l}\text { SECTION } \\
(\mathrm{KEV}-\mathrm{B})\end{array}$ & $\begin{array}{l}\text { ENERGY } \\
(\mathrm{MEV})\end{array}$ & $\begin{array}{r}\text { SECTION } \\
(\text { KEV-B) }\end{array}$ \\
\hline $1.000 \mathrm{E}-10$ & 1.963 & $8.80 \mathrm{E}-02$ & 3.91 \\
\hline $1.000 \mathrm{E}-09$ & 0.637 & $1.10 \mathrm{E}-01$ & 4.24 \\
\hline $1.000 E-08$ & 0.341 & $1.35 E-01$ & 4.85 \\
\hline $2.300 E-08$ & 0.231 & $1.60 \mathrm{E}-01$ & 6.11 \\
\hline $5.000 \mathrm{E}-08$ & 0.174 & $1.90 \mathrm{E}-01$ & 9.39 \\
\hline $7.600 \mathrm{E}-08$ & 0.141 & $2.20 \mathrm{E}-01$ & 35.58 \\
\hline $1.150 \mathrm{E}-07$ & 0.116 & $2.55 \mathrm{E}-01$ & 43.85 \\
\hline $1.700 \mathrm{E}-07$ & 0.095 & $2.90 \mathrm{E}-01$ & 18.81 \\
\hline $2.550 \mathrm{E}-07$ & 0.078 & $3.20 \mathrm{E}-01$ & 11.52 \\
\hline $3.800 E-07$ & 0.064 & $3.60 E-01$ & 9.29 \\
\hline $5.500 \mathrm{E}-07$ & 0.053 & $4.00 \mathrm{E}-01$ & 8.70 \\
\hline $8.400 \mathrm{E}-07$ & 0.043 & $4.50 \mathrm{E}-01$ & 8.28 \\
\hline $1.275 \mathrm{E}-06$ & 0.035 & $5.00 \mathrm{E}-01$ & 8.10 \\
\hline $1.900 \mathrm{E}-06$ & 0.029 & $5.50 \mathrm{E}-01$ & 8.18 \\
\hline $2.800 \mathrm{E}-06$ & 0.023 & $6.00 \mathrm{E}-01$ & 8.49 \\
\hline $4.250 \mathrm{E}-06$ & 0.019 & $6.60 \mathrm{E}-01$ & 8.99 \\
\hline $6.300 \mathrm{E}-06$ & 0.016 & $7.20 \mathrm{E}-01$ & 9.62 \\
\hline $9.200 \mathrm{E}-06$ & 0.013 & $7.80 \mathrm{E}-01$ & 10.21 \\
\hline $1.350 \mathrm{E}-05$ & 0.011 & $8.40 E-01$ & 11.22 \\
\hline $2.100 \mathrm{E}-05$ & 0.009 & $9.20 \mathrm{E}-01$ & 12.53 \\
\hline $3.000 \mathrm{E}-05$ & 0.015 & $1.00 \mathrm{E}+00$ & 14.16 \\
\hline $4.500 E-05$ & 0.020 & $1.20 E+00$ & 14.92 \\
\hline $6.900 \mathrm{E}-05$ & 0.022 & $1.40 \mathrm{E}+00$ & 14.77 \\
\hline $1.000 \mathrm{E}-04$ & 0.026 & $1.60 \mathrm{E}+00$ & 14.88 \\
\hline $1.350 \mathrm{E}-04$ & 0.031 & $1.80 \mathrm{E}+00$ & 15.23 \\
\hline $1.700 \mathrm{E}-04$ & 0.037 & $2.00 \mathrm{E}+00$ & 15.94 \\
\hline $2.200 \mathrm{E}-04$ & 0.045 & $2.30 \mathrm{E}+00$ & 16.97 \\
\hline $2.800 \mathrm{E}-04$ & 0.055 & $2.60 \mathrm{E}+00$ & 17.75 \\
\hline $3.600 \mathrm{E}-04$ & 0.068 & $2.90 \mathrm{E}+00$ & 18.33 \\
\hline $4.500 \mathrm{E}-04$ & 0.084 & $3.30 \mathrm{E}+00$ & 19.08 \\
\hline $5.750 \mathrm{E}-04$ & 0.107 & $3.70 \mathrm{E}+00$ & 20.79 \\
\hline $7.600 \mathrm{E}-04$ & 0.135 & $4.10 E+00$ & 22.35 \\
\hline $9.600 \mathrm{E}-04$ & 0.172 & $4.50 \mathrm{E}+00$ & 22.09 \\
\hline $1.275 E-03$ & 0.218 & $5.00 E+00$ & 19.70 \\
\hline $1.600 \mathrm{E}-03$ & 0.268 & $5.50 \mathrm{E}+00$ & 19.01 \\
\hline $2.000 \mathrm{E}-03$ & 0.341 & $6.00 \mathrm{E}+00$ & 18.42 \\
\hline $2.700 E-03$ & 0.431 & $6.70 \mathrm{E}+00$ & 17.20 \\
\hline $3.400 \mathrm{E}-03$ & 0.542 & $7.40 \mathrm{E}+00$ & 16.35 \\
\hline $4.500 \mathrm{E}-03$ & 0.665 & $8.20 \mathrm{E}+00$ & 16.10 \\
\hline $5.500 \mathrm{E}-03$ & 0.815 & $9.00 \mathrm{E}+00$ & 15.61 \\
\hline $7.200 E-03$ & 1.008 & $1.00 \mathrm{E}+01$ & 15.04 \\
\hline $9.200 \mathrm{E}-03$ & 1.236 & $1.10 \mathrm{E}+01$ & 14.63 \\
\hline $1.200 \mathrm{E}-02$ & 1.478 & $1.20 \mathrm{E}+01$ & 14.25 \\
\hline $1.500 \mathrm{E}-02$ & 1.738 & $1.30 \mathrm{E}+01$ & 13.75 \\
\hline
\end{tabular}




$\begin{array}{llll}1.900 \mathrm{E}-02 & 2.071 & 1.40 \mathrm{E}+01 & 13.25 \\ 2.550 \mathrm{E}-02 & 2.411 & 1.50 \mathrm{E}+01 & 12.86 \\ 3.200 \mathrm{E}-02 & 2.718 & 1.60 \mathrm{E}+01 & 12.54 \\ 4.000 \mathrm{E}-02 & 3.063 & 1.70 \mathrm{E}+01 & 12.21 \\ 5.250 \mathrm{E}-02 & 3.380 & 1.80 \mathrm{E}+01 & 11.86 \\ 6.600 \mathrm{E}-02 & 3.673 & 1.90 \mathrm{E}+01 & 11.58\end{array}$

\begin{tabular}{|c|c|c|c|}
\hline & BERYLI & & \\
\hline $\mathrm{EDL}=$ & $31.0 \mathrm{EV}$ & $\mathrm{M}=1307 . \mathrm{EV}$ & \\
\hline LOWER & CROSS & LOWER & CROSS \\
\hline ENERGY & SECTION & ENERGY & SECTION \\
\hline$(\mathrm{MEV})$ & $(\mathrm{KEV}-\mathrm{B})$ & $(\mathrm{MEV})$ & $(\mathrm{KEV}-\mathrm{B})$ \\
\hline 1.000E-10 & 0.095 & $8.80 E-02$ & 31.02 \\
\hline $1.000 \mathrm{E}-09$ & 0.030 & $1.10 \mathrm{E}-01$ & 33.67 \\
\hline $1.000 \mathrm{E}-08$ & 0.016 & $1.35 \mathrm{E}-01$ & 35.69 \\
\hline $2.300 E-08$ & 0.011 & $1.60 \mathrm{E}-01$ & 37.16 \\
\hline $5.000 E-08$ & 0.008 & $1.90 \mathrm{E}-01$ & 38.07 \\
\hline $7.600 \mathrm{E}-08$ & 0.006 & $2.20 \mathrm{E}-01$ & 38.44 \\
\hline $1.150 \mathrm{E}-07$ & 0.005 & $2.55 \mathrm{E}-01$ & 38.85 \\
\hline $1.700 \mathrm{E}-07$ & 0.004 & $2.90 \mathrm{E}-01$ & 39.40 \\
\hline $2.550 \mathrm{E}-07$ & 0.004 & $3.20 \mathrm{E}-01$ & 39.57 \\
\hline $3.800 E-07$ & 0.003 & $3.60 \mathrm{E}-01$ & 39.53 \\
\hline $5.500 \mathrm{E}-07$ & 0.002 & $4.00 \mathrm{E}-01$ & 39.16 \\
\hline $8.400 \mathrm{E}-07$ & 0.002 & $4.50 \mathrm{E}-01$ & 38.43 \\
\hline $1.275 E-06$ & 0.002 & $5.00 \mathrm{E}-01$ & 38.65 \\
\hline $1.900 \mathrm{E}-06$ & 0.001 & $5.50 \mathrm{E}-01$ & 43.03 \\
\hline $2.800 E-06$ & 0.001 & $6.00 \mathrm{E}-01$ & 65.51 \\
\hline $4.250 E-06$ & 0.001 & $6.60 \mathrm{E}-01$ & 42.34 \\
\hline $6.300 E-06$ & 0.001 & $7.20 \mathrm{E}-01$ & 41.31 \\
\hline $9.200 \mathrm{E}-06$ & 0.001 & $7.80 \mathrm{E}-01$ & 46.31 \\
\hline $1.350 \mathrm{E}-05$ & 0.000 & $8.40 \mathrm{E}-01$ & 42.36 \\
\hline $2.100 \mathrm{E}-05$ & 0.000 & $9.20 \mathrm{E}-01$ & 41.48 \\
\hline $3.000 \mathrm{E}-05$ & 0.000 & $1.00 \mathrm{E}+00$ & 39.20 \\
\hline $4.500 \mathrm{E}-05$ & 0.000 & $1.20 \mathrm{E}+00$ & 34.04 \\
\hline $6.900 \mathrm{E}-05$ & 0.000 & $1.40 \mathrm{E}+00$ & 28.99 \\
\hline $1.000 \mathrm{E}-04$ & 0.091 & $1.60 \mathrm{E}+00$ & 25.54 \\
\hline $1.350 \mathrm{E}-04$ & 0.175 & $1.80 \mathrm{E}+00$ & 23.85 \\
\hline $1.700 \mathrm{E}-04$ & 0.236 & $2.00 \mathrm{E}+00$ & 26.34 \\
\hline $2.200 \mathrm{E}-04$ & 0.260 & $2.30 \mathrm{E}+00$ & 35.75 \\
\hline $2.800 \mathrm{E}-04$ & 0.304 & $2.60 \mathrm{E}+00$ & 49.54 \\
\hline $3.600 \mathrm{E}-04$ & 0.358 & $2.90 \mathrm{E}+00$ & 40.27 \\
\hline $4.500 \mathrm{E}-04$ & 0.434 & $3.30 \mathrm{E}+00$ & 33.02 \\
\hline $5.750 \mathrm{E}-04$ & 0.542 & $3.70 \mathrm{E}+00$ & 28.85 \\
\hline $7.600 \mathrm{E}-04$ & 0.680 & $4.10 \mathrm{E}+00$ & 28.49 \\
\hline $9.600 \mathrm{E}-04$ & 0.867 & $4.50 \mathrm{E}+00$ & 28.56 \\
\hline $1.275 E-03$ & 1.094 & $5.00 \mathrm{E}+00$ & 28.45 \\
\hline $1.600 \mathrm{E}-03$ & 1.346 & $5.50 \mathrm{E}+00$ & 28.11 \\
\hline $2.000 E-03$ & 1.725 & $6.00 \mathrm{E}+00$ & 27.77 \\
\hline $2.700 E-03$ & 2.197 & $6.70 \mathrm{E}+00$ & 27.31 \\
\hline $3.400 E-03$ & 2.787 & $7.40 \mathrm{E}+00$ & 26.97 \\
\hline $4.500 E-03$ & 3.457 & $8.20 \mathrm{E}+00$ & 26.61 \\
\hline $5.500 E-03$ & 4.286 & $9.00 \mathrm{E}+00$ & 26.17 \\
\hline $7.200 \mathrm{E}-03$ & 5.379 & $1.00 \mathrm{E}+01$ & 25.68 \\
\hline $9.200 \mathrm{E}-03$ & 6.726 & $1.10 \mathrm{E}+01$ & 25.13 \\
\hline $1.200 \mathrm{E}-02$ & 8.246 & $1.20 \mathrm{E}+01$ & 24.50 \\
\hline
\end{tabular}




$\begin{array}{rrrr}1.500 \mathrm{E}-02 & 9.959 & 1.30 \mathrm{E}+01 & 23.95 \\ 1.900 \mathrm{E}-02 & 12.315 & 1.40 \mathrm{E}+01 & 23.22 \\ 2.550 \mathrm{E}-02 & 14.942 & 1.50 \mathrm{E}+01 & 22.79 \\ 3.200 \mathrm{E}-02 & 17.546 & 1.60 \mathrm{E}+01 & 21.99 \\ 4.000 \mathrm{E}-02 & 20.771 & 1.70 \mathrm{E}+01 & 21.99 \\ 5.250 \mathrm{E}-02 & 24.119 & 1.80 \mathrm{E}+01 & 21.86 \\ 6.600 \mathrm{E}-02 & 27.699 & 1.90 \mathrm{E}+01 & 21.42\end{array}$

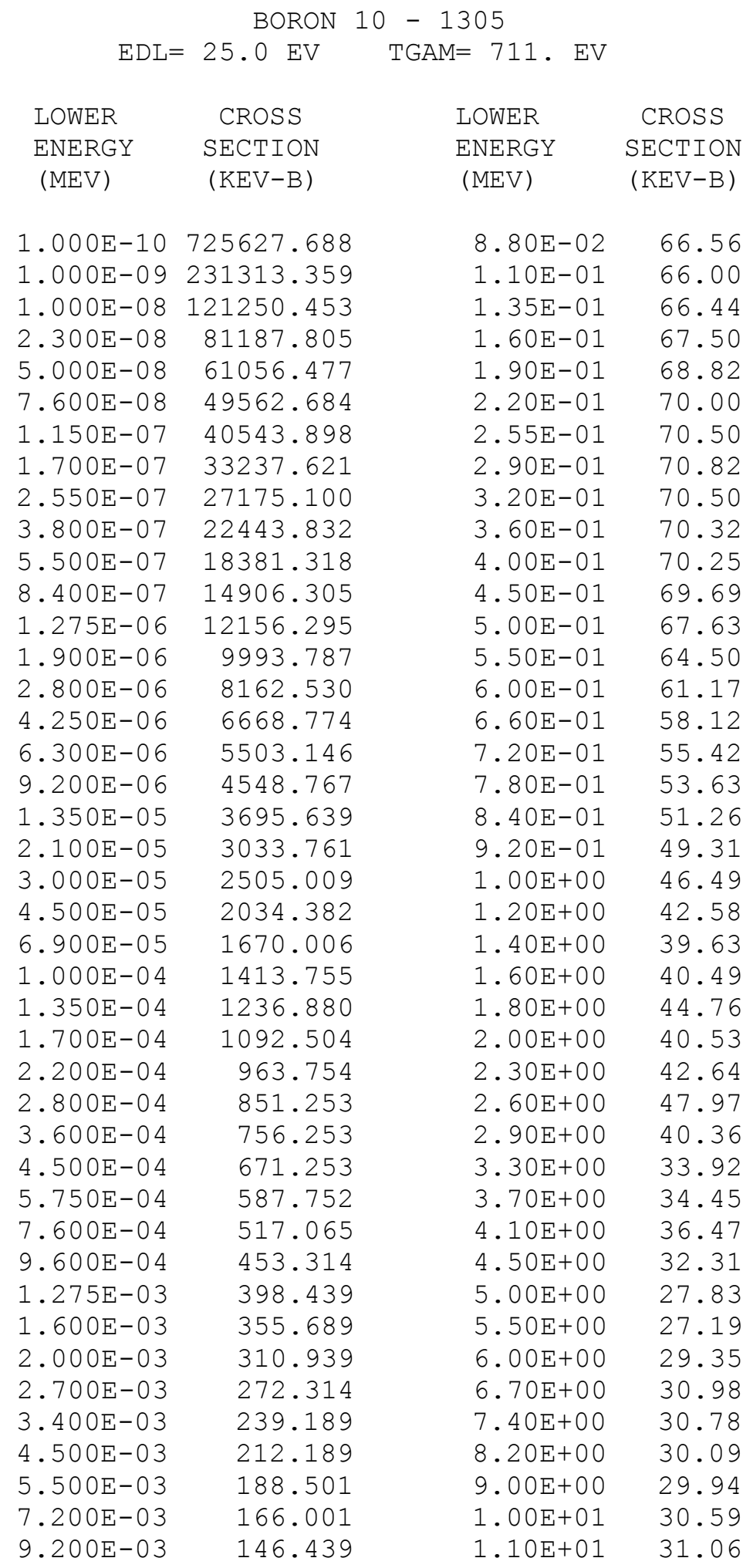




$\begin{array}{rrrr}1.200 \mathrm{E}-02 & 130.376 & 1.20 \mathrm{E}+01 & 30.98 \\ 1.500 \mathrm{E}-02 & 117.064 & 1.30 \mathrm{E}+01 & 30.86 \\ 1.900 \mathrm{E}-02 & 103.876 & 1.40 \mathrm{E}+01 & 30.86 \\ 2.550 \mathrm{E}-02 & 93.126 & 1.50 \mathrm{E}+01 & 30.90 \\ 3.200 \mathrm{E}-02 & 85.314 & 1.60 \mathrm{E}+01 & 30.84 \\ 4.000 \mathrm{E}-02 & 78.251 & 1.70 \mathrm{E}+01 & 30.83 \\ 5.250 \mathrm{E}-02 & 72.814 & 1.80 \mathrm{E}+01 & 30.81 \\ 6.600 \mathrm{E}-02 & 68.814 & 1.90 \mathrm{E}+01 & 30.77\end{array}$

\begin{tabular}{lccc}
\multicolumn{5}{c}{ BORON $11-1160$} \\
& & -11 & \\
LOWER & CROSS & LOWER & CROSS \\
ENERGY & SECTION & ENERGY & SECTION \\
(MEV) & (KEV-B) & (MEV) & $($ KEV-B) \\
1.000E-10 & 0.153 & $8.80 \mathrm{E}-02$ & 27.71 \\
$1.000 \mathrm{E}-09$ & 0.048 & $1.10 \mathrm{E}-01$ & 30.70 \\
$1.000 \mathrm{E}-08$ & 0.025 & $1.35 \mathrm{E}-01$ & 33.11 \\
$2.300 \mathrm{E}-08$ & 0.017 & $1.60 \mathrm{E}-01$ & 35.26 \\
$5.000 \mathrm{E}-08$ & 0.013 & $1.90 \mathrm{E}-01$ & 37.36 \\
$7.600 \mathrm{E}-08$ & 0.010 & $2.20 \mathrm{E}-01$ & 39.31 \\
$1.150 \mathrm{E}-07$ & 0.008 & $2.55 \mathrm{E}-01$ & 40.02 \\
$1.700 \mathrm{E}-07$ & 0.007 & $2.90 \mathrm{E}-01$ & 40.53 \\
$2.550 \mathrm{E}-07$ & 0.006 & $3.20 \mathrm{E}-01$ & 41.33 \\
$3.800 \mathrm{E}-07$ & 0.005 & $3.60 \mathrm{E}-01$ & 44.90 \\
$5.500 \mathrm{E}-07$ & 0.004 & $4.00 \mathrm{E}-01$ & 76.56 \\
$8.400 \mathrm{E}-07$ & 0.003 & $4.50 \mathrm{E}-01$ & 59.78 \\
$1.275 \mathrm{E}-06$ & 0.003 & $5.00 \mathrm{E}-01$ & 48.72 \\
$1.900 \mathrm{E}-06$ & 0.002 & $5.50 \mathrm{E}-01$ & 46.32 \\
$2.800 \mathrm{E}-06$ & 0.002 & $6.00 \mathrm{E}-01$ & 45.06 \\
$4.250 \mathrm{E}-06$ & 0.001 & $6.60 \mathrm{E}-01$ & 44.05 \\
$6.300 \mathrm{E}-06$ & 0.001 & $7.20 \mathrm{E}-01$ & 43.53 \\
$9.200 \mathrm{E}-06$ & 0.001 & $7.80 \mathrm{E}-01$ & 43.26 \\
$1.350 \mathrm{E}-05$ & 0.001 & $8.40 \mathrm{E}-01$ & 42.43 \\
$2.100 \mathrm{E}-05$ & 0.001 & $9.20 \mathrm{E}-01$ & 41.03 \\
$3.000 \mathrm{E}-05$ & 0.001 & $1.00 \mathrm{E}+00$ & 41.74 \\
$4.500 \mathrm{E}-05$ & 0.000 & $1.20 \mathrm{E}+00$ & 62.43 \\
$6.900 \mathrm{E}-05$ & 0.000 & $1.40 \mathrm{E}+00$ & 45.70 \\
$1.000 \mathrm{E}-04$ & 0.077 & $1.60 \mathrm{E}+00$ & 43.95 \\
$1.350 \mathrm{E}-04$ & 0.131 & $1.80 \mathrm{E}+00$ & 40.50 \\
$1.700 \mathrm{E}-04$ & 0.163 & $2.00 \mathrm{E}+00$ & 35.35 \\
$2.200 \mathrm{E}-04$ & 0.184 & $2.30 \mathrm{E}+00$ & 37.99 \\
$2.800 \mathrm{E}-04$ & 0.218 & $2.60 \mathrm{E}+00$ & 35.51 \\
$3.600 \mathrm{E}-04$ & 0.261 & $2.90 \mathrm{E}+00$ & 31.89 \\
$4.500 \mathrm{E}-04$ & 0.315 & $3.30 \mathrm{E}+00$ & 34.24 \\
$5.750 \mathrm{E}-04$ & 0.397 & $3.70 \mathrm{E}+00$ & 32.39 \\
$7.600 \mathrm{E}-04$ & 0.500 & $4.10 \mathrm{E}+00$ & 29.43 \\
$9.600 \mathrm{E}-04$ & 0.640 & $4.50 \mathrm{E}+00$ & 37.35 \\
$1.275 \mathrm{E}-03$ & 0.808 & $5.00 \mathrm{E}+00$ & 36.88 \\
$1.600 \mathrm{E}-03$ & 1.001 & $5.50 \mathrm{E}+00$ & 36.73 \\
$2.000 \mathrm{E}-03$ & 1.286 & $6.00 \mathrm{E}+00$ & 35.26 \\
$2.700 \mathrm{E}-03$ & 1.643 & $6.70 \mathrm{E}+00$ & 33.04 \\
$3.400 \mathrm{E}-03$ & 2.090 & $7.40 \mathrm{E}+00$ & 33.68 \\
$4.500 \mathrm{E}-03$ & 2.604 & $8.20 \mathrm{E}+00$ & 30.69 \\
$5.500 \mathrm{E}-03$ & 3.245 & $9.00 \mathrm{E}+00$ & 30.47 \\
$7.200 \mathrm{E}-03$ & 4.100 & $1.00 \mathrm{E}+01$ & 30.43
\end{tabular}




$\begin{array}{rrrr}9.200 \mathrm{E}-03 & 5.182 & 1.10 \mathrm{E}+01 & 30.53 \\ 1.200 \mathrm{E}-02 & 6.463 & 1.20 \mathrm{E}+01 & 31.23 \\ 1.500 \mathrm{E}-02 & 7.957 & 1.30 \mathrm{E}+01 & 33.18 \\ 1.900 \mathrm{E}-02 & 11.417 & 1.40 \mathrm{E}+01 & 33.42 \\ 2.550 \mathrm{E}-02 & 12.219 & 1.50 \mathrm{E}+01 & 33.20 \\ 3.200 \mathrm{E}-02 & 14.394 & 1.60 \mathrm{E}+01 & 32.83 \\ 4.000 \mathrm{E}-02 & 17.156 & 1.70 \mathrm{E}+01 & 32.46 \\ 5.250 \mathrm{E}-02 & 20.238 & 1.80 \mathrm{E}+01 & 32.09 \\ 6.600 \mathrm{E}-02 & 23.881 & 1.90 \mathrm{E}+01 & 31.70 \\ & & 2.00 \mathrm{E}+01 & \end{array}$

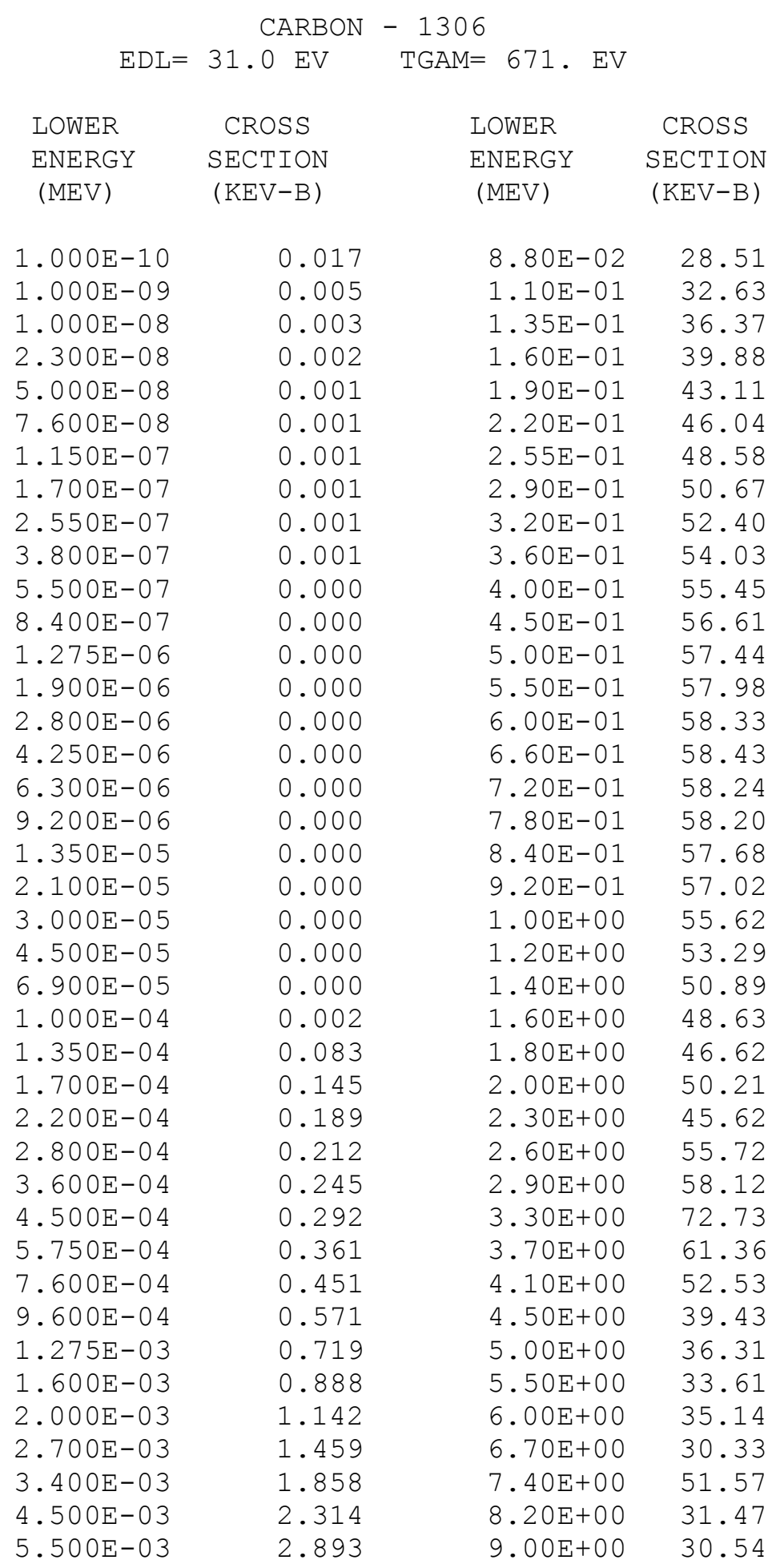




$\begin{array}{rrrr}7.200 \mathrm{E}-03 & 3.661 & 1.00 \mathrm{E}+01 & 33.43 \\ 9.200 \mathrm{E}-03 & 4.629 & 1.10 \mathrm{E}+01 & 39.79 \\ 1.200 \mathrm{E}-02 & 5.761 & 1.20 \mathrm{E}+01 & 39.13 \\ 1.500 \mathrm{E}-02 & 7.072 & 1.30 \mathrm{E}+01 & 38.51 \\ 1.900 \mathrm{E}-02 & 8.944 & 1.40 \mathrm{E}+01 & 39.11 \\ 2.550 \mathrm{E}-02 & 11.129 & 1.50 \mathrm{E}+01 & 43.03 \\ 3.200 \mathrm{E}-02 & 13.415 & 1.60 \mathrm{E}+01 & 44.28 \\ 4.000 \mathrm{E}-02 & 16.399 & 1.70 \mathrm{E}+01 & 42.79 \\ 5.250 \mathrm{E}-02 & 19.848 & 1.80 \mathrm{E}+01 & 45.59 \\ 6.600 \mathrm{E}-02 & 24.017 & 1.90 \mathrm{E}+01 & 46.58 \\ & & 2.00 \mathrm{E}+01 & \end{array}$

\begin{tabular}{|c|c|c|c|}
\hline & NITRO & 275 & \\
\hline $\mathrm{EDL}=$ & $30.0 \mathrm{EV}$ & $\mathrm{TGAM}=1659 . \mathrm{EV}$ & \\
\hline LOWER & CROSS & LOWER & CROSS \\
\hline ENERGY & SECTION & ENERGY & SECTION \\
\hline$(\mathrm{MEV})$ & $(\mathrm{KEV}-\mathrm{B})$ & $(\mathrm{MEV})$ & $(\mathrm{KEV}-\mathrm{B})$ \\
\hline $1.000 \mathrm{E}-10$ & 0.951 & $8.80 E-02$ & 28.47 \\
\hline $1.000 \mathrm{E}-09$ & 0.301 & $1.10 \mathrm{E}-01$ & 31.58 \\
\hline $1.000 E-08$ & 0.157 & $1.35 E-01$ & 34.46 \\
\hline $2.300 E-08$ & 0.105 & $1.60 \mathrm{E}-01$ & 37.16 \\
\hline $5.000 \mathrm{E}-08$ & 0.079 & $1.90 \mathrm{E}-01$ & 39.63 \\
\hline $7.600 \mathrm{E}-08$ & 0.064 & $2.20 E-01$ & 41.93 \\
\hline $1.150 \mathrm{E}-07$ & 0.053 & $2.55 \mathrm{E}-01$ & 43.67 \\
\hline $1.700 \mathrm{E}-07$ & 0.043 & $2.90 \mathrm{E}-01$ & 45.14 \\
\hline $2.550 \mathrm{E}-07$ & 0.035 & $3.20 \mathrm{E}-01$ & 46.28 \\
\hline $3.800 \mathrm{E}-07$ & 0.029 & $3.60 \mathrm{E}-01$ & 47.75 \\
\hline $5.500 \mathrm{E}-07$ & 0.024 & $4.00 \mathrm{E}-01$ & 62.44 \\
\hline $8.400 \mathrm{E}-07$ & 0.019 & $4.50 \mathrm{E}-01$ & 46.17 \\
\hline $1.275 \mathrm{E}-06$ & 0.016 & $5.00 \mathrm{E}-01$ & 42.42 \\
\hline $1.900 \mathrm{E}-06$ & 0.013 & $5.50 \mathrm{E}-01$ & 38.66 \\
\hline $2.800 E-06$ & 0.011 & $6.00 \mathrm{E}-01$ & 41.44 \\
\hline $4.250 E-06$ & 0.009 & $6.60 \mathrm{E}-01$ & 51.74 \\
\hline $6.300 E-06$ & 0.007 & $7.20 \mathrm{E}-01$ & 46.62 \\
\hline $9.200 \mathrm{E}-06$ & 0.006 & $7.80 \mathrm{E}-01$ & 43.20 \\
\hline $1.350 \mathrm{E}-05$ & 0.005 & $8.40 E-01$ & 37.29 \\
\hline $2.100 \mathrm{E}-05$ & 0.004 & $9.20 \mathrm{E}-01$ & 32.06 \\
\hline $3.000 \mathrm{E}-05$ & 0.003 & $1.00 \mathrm{E}+00$ & 56.39 \\
\hline $4.500 \mathrm{E}-05$ & 0.003 & $1.20 \mathrm{E}+00$ & 56.05 \\
\hline $6.900 \mathrm{E}-05$ & 0.002 & $1.40 \mathrm{E}+00$ & 61.58 \\
\hline $1.000 \mathrm{E}-04$ & 0.002 & $1.60 \mathrm{E}+00$ & 63.26 \\
\hline $1.350 \mathrm{E}-04$ & 0.114 & $1.80 \mathrm{E}+00$ & 52.72 \\
\hline $1.700 \mathrm{E}-04$ & 0.248 & $2.00 \mathrm{E}+00$ & 52.89 \\
\hline $2.200 \mathrm{E}-04$ & 0.345 & $2.30 \mathrm{E}+00$ & 48.23 \\
\hline $2.800 \mathrm{E}-04$ & 0.383 & $2.60 \mathrm{E}+00$ & 50.57 \\
\hline $3.600 \mathrm{E}-04$ & 0.439 & $2.90 \mathrm{E}+00$ & 64.79 \\
\hline $4.500 \mathrm{E}-04$ & 0.509 & $3.30 \mathrm{E}+00$ & 65.89 \\
\hline $5.750 \mathrm{E}-04$ & 0.621 & $3.70 \mathrm{E}+00$ & 74.37 \\
\hline $7.600 \mathrm{E}-04$ & 0.768 & $4.10 \mathrm{E}+00$ & 73.68 \\
\hline $9.600 \mathrm{E}-04$ & 0.954 & $4.50 \mathrm{E}+00$ & 45.71 \\
\hline $1.275 E-03$ & 1.185 & $5.00 \mathrm{E}+00$ & 55.62 \\
\hline $1.600 \mathrm{E}-03$ & 1.434 & $5.50 \mathrm{E}+00$ & 54.88 \\
\hline $2.000 \mathrm{E}-03$ & 1.809 & $6.00 \mathrm{E}+00$ & 52.10 \\
\hline $2.700 \mathrm{E}-03$ & 2.270 & $6.70 \mathrm{E}+00$ & 53.29 \\
\hline $3.400 E-03$ & 2.846 & $7.40 \mathrm{E}+00$ & 60.41 \\
\hline $4.500 E-03$ & 3.497 & $8.20 \mathrm{E}+00$ & 50.92 \\
\hline
\end{tabular}




$\begin{array}{lrll}5.500 \mathrm{E}-03 & 4.302 & 9.00 \mathrm{E}+00 & 53.39 \\ 7.200 \mathrm{E}-03 & 5.370 & 1.00 \mathrm{E}+01 & 58.08 \\ 9.200 \mathrm{E}-03 & 6.684 & 1.10 \mathrm{E}+01 & 58.47 \\ 1.200 \mathrm{E}-02 & 8.175 & 1.20 \mathrm{E}+01 & 63.73 \\ 1.500 \mathrm{E}-02 & 9.803 & 1.30 \mathrm{E}+01 & 63.51 \\ 1.900 \mathrm{E}-02 & 11.970 & 1.40 \mathrm{E}+01 & 63.18 \\ 2.550 \mathrm{E}-02 & 14.378 & 1.50 \mathrm{E}+01 & 62.94 \\ 3.200 \mathrm{E}-02 & 16.665 & 1.60 \mathrm{E}+01 & 62.08 \\ 4.000 \mathrm{E}-02 & 19.373 & 1.70 \mathrm{E}+01 & 61.24 \\ 5.250 \mathrm{E}-02 & 22.140 & 1.80 \mathrm{E}+01 & 60.54 \\ 6.600 \mathrm{E}-02 & 25.283 & 1.90 \mathrm{E}+01 & 59.83 \\ & & 2.00 \mathrm{E}+01 & \end{array}$

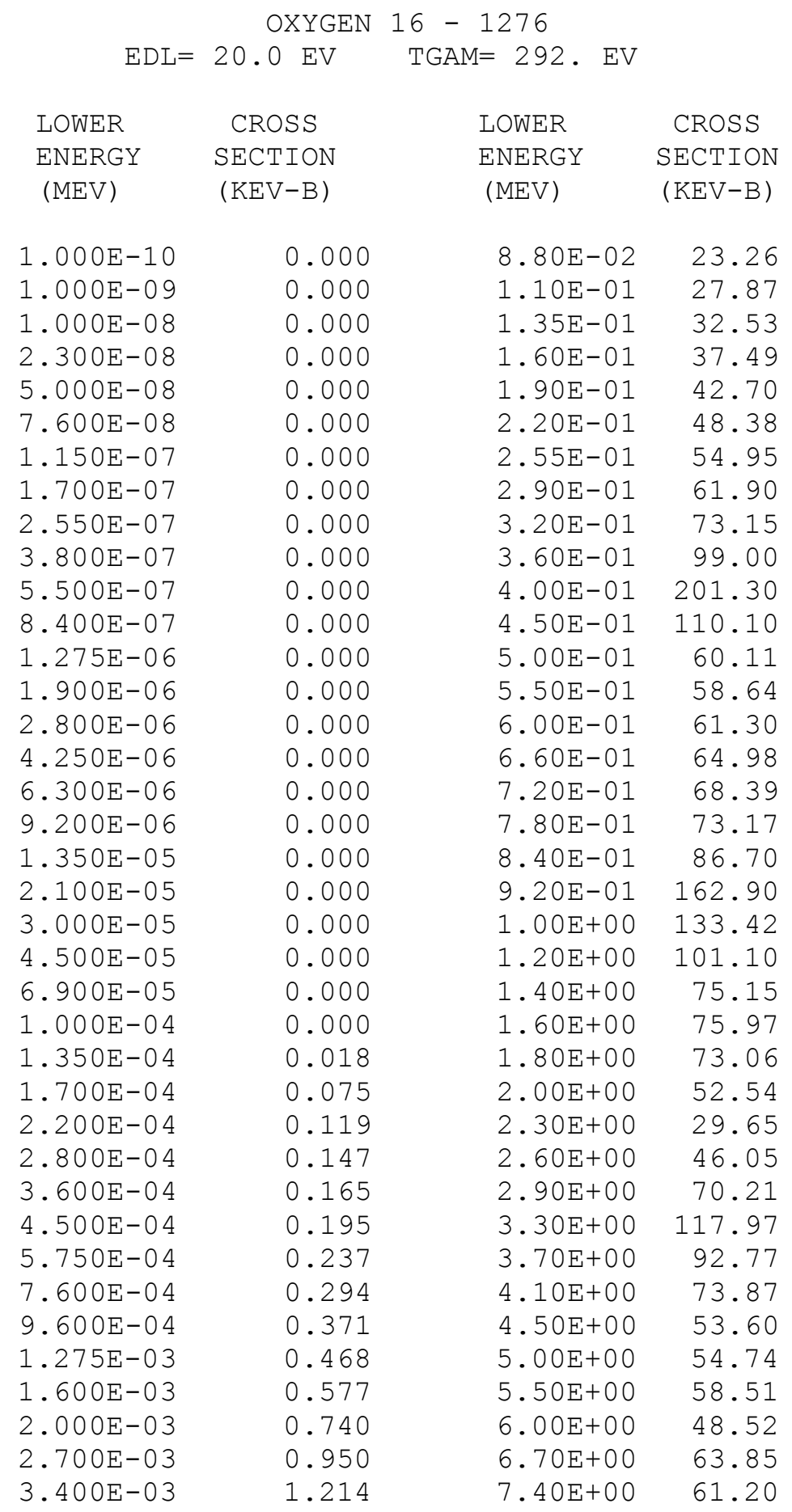




$\begin{array}{lrll}4.500 \mathrm{E}-03 & 1.520 & 8.20 \mathrm{E}+00 & 67.20 \\ 5.500 \mathrm{E}-03 & 1.906 & 9.00 \mathrm{E}+00 & 64.77 \\ 7.200 \mathrm{E}-03 & 2.429 & 1.00 \mathrm{E}+01 & 69.82 \\ 9.200 \mathrm{E}-03 & 3.093 & 1.10 \mathrm{E}+01 & 86.62 \\ 1.200 \mathrm{E}-02 & 3.882 & 1.20 \mathrm{E}+01 & 78.15 \\ 1.500 \mathrm{E}-02 & 4.817 & 1.30 \mathrm{E}+01 & 79.95 \\ 1.900 \mathrm{E}-02 & 6.181 & 1.40 \mathrm{E}+01 & 83.55 \\ 2.550 \mathrm{E}-02 & 7.823 & 1.50 \mathrm{E}+01 & 82.43 \\ 3.200 \mathrm{E}-02 & 9.592 & 1.60 \mathrm{E}+01 & 80.70 \\ 4.000 \mathrm{E}-02 & 12.015 & 1.70 \mathrm{E}+01 & 82.95 \\ 5.250 \mathrm{E}-02 & 14.955 & 1.80 \mathrm{E}+01 & 81.60 \\ 6.600 \mathrm{E}-02 & 18.787 & 1.90 \mathrm{E}+01 & 82.35 \\ & & 2.00 \mathrm{E}+01 & \end{array}$

\begin{tabular}{lccr}
\multicolumn{5}{c}{ EDL= 30.0 EV } & TGAM=1207. EV & \\
LOWER & CROSS & \multicolumn{1}{l}{ LOWER } & \multicolumn{1}{c}{ CROSS } \\
ENERGY & SECTION & ENERGY & SECTION \\
(MEV) & (KEV-B) & (MEV) & $(\mathrm{KEV}-\mathrm{B})$ \\
& & & \\
$1.000 \mathrm{E}-10$ & 0.088 & $8.80 \mathrm{E}-02$ & 94.07 \\
$1.000 \mathrm{E}-09$ & 0.028 & $1.10 \mathrm{E}-01$ & 39.31 \\
$1.000 \mathrm{E}-08$ & 0.014 & $1.35 \mathrm{E}-01$ & 31.37 \\
$2.300 \mathrm{E}-08$ & 0.010 & $1.60 \mathrm{E}-01$ & 32.17 \\
$5.000 \mathrm{E}-08$ & 0.007 & $1.90 \mathrm{E}-01$ & 38.66 \\
$7.600 \mathrm{E}-08$ & 0.006 & $2.20 \mathrm{E}-01$ & 62.90 \\
$1.150 \mathrm{E}-07$ & 0.005 & $2.55 \mathrm{E}-01$ & 108.70 \\
$1.700 \mathrm{E}-07$ & 0.004 & $2.90 \mathrm{E}-01$ & 108.01 \\
$2.550 \mathrm{E}-07$ & 0.003 & $3.20 \mathrm{E}-01$ & 109.13 \\
$3.800 \mathrm{E}-07$ & 0.003 & $3.60 \mathrm{E}-01$ & 115.66 \\
$5.500 \mathrm{E}-07$ & 0.002 & $4.00 \mathrm{E}-01$ & 117.60 \\
$8.400 \mathrm{E}-07$ & 0.002 & $4.50 \mathrm{E}-01$ & 85.96 \\
$1.275 \mathrm{E}-06$ & 0.001 & $5.00 \mathrm{E}-01$ & 88.88 \\
$1.900 \mathrm{E}-06$ & 0.001 & $5.50 \mathrm{E}-01$ & 84.99 \\
$2.800 \mathrm{E}-06$ & 0.001 & $6.00 \mathrm{E}-01$ & 80.63 \\
$4.250 \mathrm{E}-06$ & 0.001 & $6.60 \mathrm{E}-01$ & 68.39 \\
$6.300 \mathrm{E}-06$ & 0.001 & $7.20 \mathrm{E}-01$ & 92.40 \\
$9.200 \mathrm{E}-06$ & 0.001 & $7.80 \mathrm{E}-01$ & 96.60 \\
$1.350 \mathrm{E}-05$ & 0.000 & $8.40 \mathrm{E}-01$ & 94.88 \\
$2.100 \mathrm{E}-05$ & 0.000 & $9.20 \mathrm{E}-01$ & 92.63 \\
$3.000 \mathrm{E}-05$ & 0.000 & $1.00 \mathrm{E}+00$ & 99.45 \\
$4.500 \mathrm{E}-05$ & 0.000 & $1.20 \mathrm{E}+00$ & 125.41 \\
$6.900 \mathrm{E}-05$ & 0.000 & $1.40 \mathrm{E}+00$ & 111.76 \\
$1.000 \mathrm{E}-04$ & 0.000 & $1.60 \mathrm{E}+00$ & 119.10 \\
$1.350 \mathrm{E}-04$ & 0.000 & $1.80 \mathrm{E}+00$ & 113.40 \\
$1.700 \mathrm{E}-04$ & 0.042 & $2.00 \mathrm{E}+00$ & 114.98 \\
$2.200 \mathrm{E}-04$ & 0.094 & $2.30 \mathrm{E}+00$ & 123.38 \\
$2.800 \mathrm{E}-04$ & 0.132 & $2.60 \mathrm{E}+00$ & 122.26 \\
$3.600 \mathrm{E}-04$ & 0.150 & $2.90 \mathrm{E}+00$ & 105.91 \\
$4.500 \mathrm{E}-04$ & 0.171 & $3.30 \mathrm{E}+00$ & 98.56 \\
$5.750 \mathrm{E}-04$ & 0.208 & $3.70 \mathrm{E}+00$ & 87.68 \\
$7.600 \mathrm{E}-04$ & 0.254 & $4.10 \mathrm{E}+00$ & 98.86 \\
$9.600 \mathrm{E}-04$ & 0.320 & $4.50 \mathrm{E}+00$ & 93.91 \\
$1.275 \mathrm{E}-03$ & 0.401 & $5.00 \mathrm{E}+00$ & 90.46 \\
$1.600 \mathrm{E}-03$ & 0.493 & $5.50 \mathrm{E}+00$ & 101.03 \\
$2.000 \mathrm{E}-03$ & 0.634 & $6.00 \mathrm{E}+00$ & 93.76 \\
$2.700 \mathrm{E}-03$ & 0.812 & $6.70 \mathrm{E}+00$ & 94.73
\end{tabular}




$\begin{array}{rrrr}3.400 \mathrm{E}-03 & 1.039 & 7.40 \mathrm{E}+00 & 99.53 \\ 4.500 \mathrm{E}-03 & 1.301 & 8.20 \mathrm{E}+00 & 102.46 \\ 5.500 \mathrm{E}-03 & 1.634 & 9.00 \mathrm{E}+00 & 103.21 \\ 7.200 \mathrm{E}-03 & 2.081 & 1.00 \mathrm{E}+01 & 106.13 \\ 9.200 \mathrm{E}-03 & 2.663 & 1.10 \mathrm{E}+01 & 106.36 \\ 1.200 \mathrm{E}-02 & 3.355 & 1.20 \mathrm{E}+01 & 107.93 \\ 1.500 \mathrm{E}-02 & 4.177 & 1.30 \mathrm{E}+01 & 108.76 \\ 1.900 \mathrm{E}-02 & 5.626 & 1.40 \mathrm{E}+01 & 110.33 \\ 2.550 \mathrm{E}-02 & 26.458 & 1.50 \mathrm{E}+01 & 111.76 \\ 3.200 \mathrm{E}-02 & 9.084 & 1.60 \mathrm{E}+01 & 111.61 \\ 4.000 \mathrm{E}-02 & 34.454 & 1.70 \mathrm{E}+01 & 110.86 \\ 5.250 \mathrm{E}-02 & 13.120 & 1.80 \mathrm{E}+01 & 110.48 \\ 6.600 \mathrm{E}-02 & 21.836 & 1.90 \mathrm{E}+01 & 109.82 \\ & & 2.00 \mathrm{E}+01 & \end{array}$

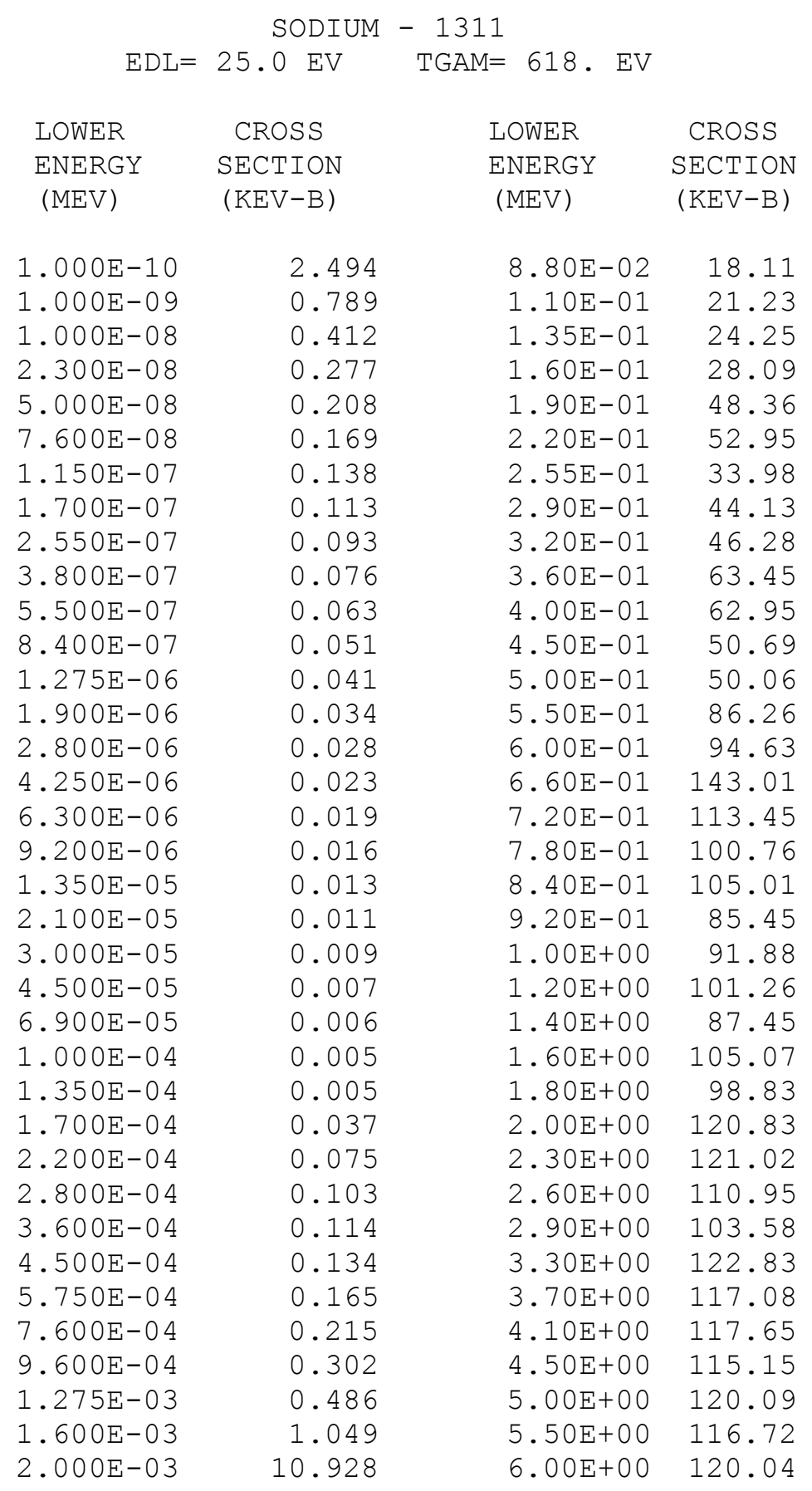




$\begin{array}{rrrr}2.700 \mathrm{E}-03 & 37.288 & 6.70 \mathrm{E}+00 & 114.29 \\ 3.400 \mathrm{E}-03 & 5.712 & 7.40 \mathrm{E}+00 & 121.42 \\ 4.500 \mathrm{E}-03 & 3.080 & 8.20 \mathrm{E}+00 & 118.55 \\ 5.500 \mathrm{E}-03 & 2.727 & 9.00 \mathrm{E}+00 & 120.43 \\ 7.200 \mathrm{E}-03 & 2.829 & 1.00 \mathrm{E}+01 & 128.50 \\ 9.200 \mathrm{E}-03 & 3.185 & 1.10 \mathrm{E}+01 & 133.82 \\ 1.200 \mathrm{E}-02 & 3.696 & 1.20 \mathrm{E}+01 & 136.71 \\ 1.500 \mathrm{E}-02 & 4.353 & 1.30 \mathrm{E}+01 & 138.78 \\ 1.900 \mathrm{E}-02 & 5.368 & 1.40 \mathrm{E}+01 & 139.98 \\ 2.550 \mathrm{E}-02 & 6.675 & 1.50 \mathrm{E}+01 & 140.93 \\ 3.200 \mathrm{E}-02 & 8.172 & 1.60 \mathrm{E}+01 & 142.19 \\ 4.000 \mathrm{E}-02 & 11.814 & 1.70 \mathrm{E}+01 & 143.95 \\ 5.250 \mathrm{E}-02 & 22.187 & 1.80 \mathrm{E}+01 & 145.34 \\ 6.600 \mathrm{E}-02 & 14.994 & 1.90 \mathrm{E}+01 & 146.47 \\ & & 2.00 \mathrm{E}+01 & \end{array}$

\begin{tabular}{|c|c|c|c|}
\hline & MAGNES & & \\
\hline $\mathrm{EDL}=$ & $25.0 \mathrm{EV}$ & $\mathrm{M}=574 . \mathrm{EV}$ & \\
\hline LOWER & CROSS & LOWER & CROSS \\
\hline $\begin{array}{l}\text { ENERGY } \\
\text { (MEV) }\end{array}$ & $\begin{array}{c}\text { SECTION } \\
(\mathrm{KEV}-\mathrm{B})\end{array}$ & $\begin{array}{l}\text { ENERGY } \\
(\mathrm{MEV})\end{array}$ & $\begin{array}{c}\text { SECTION } \\
(\mathrm{KEV}-\mathrm{B})\end{array}$ \\
\hline $1.000 \mathrm{E}-10$ & 0.277 & $8.80 E-02$ & 51.94 \\
\hline $1.000 E-09$ & 0.087 & $1.10 \mathrm{E}-01$ & 27.88 \\
\hline $1.000 E-08$ & 0.046 & $1.35 E-01$ & 29.98 \\
\hline $2.300 E-08$ & 0.031 & $1.60 \mathrm{E}-01$ & 34.36 \\
\hline $5.000 E-08$ & 0.023 & $1.90 \mathrm{E}-01$ & 51.18 \\
\hline $7.600 \mathrm{E}-08$ & 0.019 & $2.20 \mathrm{E}-01$ & 86.06 \\
\hline $1.150 \mathrm{E}-07$ & 0.015 & $2.55 \mathrm{E}-01$ & 119.06 \\
\hline $1.700 \mathrm{E}-07$ & 0.013 & $2.90 \mathrm{E}-01$ & 99.69 \\
\hline $2.550 \mathrm{E}-07$ & 0.010 & $3.20 \mathrm{E}-01$ & 77.75 \\
\hline $3.800 \mathrm{E}-07$ & 0.009 & $3.60 \mathrm{E}-01$ & 66.25 \\
\hline $5.500 \mathrm{E}-07$ & 0.007 & $4.00 \mathrm{E}-01$ & 132.25 \\
\hline $8.400 \mathrm{E}-07$ & 0.006 & $4.50 \mathrm{E}-01$ & 82.72 \\
\hline $1.275 \mathrm{E}-06$ & 0.005 & $5.00 \mathrm{E}-01$ & 69.22 \\
\hline $1.900 \mathrm{E}-06$ & 0.004 & $5.50 \mathrm{E}-01$ & 69.53 \\
\hline $2.800 E-06$ & 0.003 & $6.00 \mathrm{E}-01$ & 46.72 \\
\hline $4.250 \mathrm{E}-06$ & 0.003 & $6.60 \mathrm{E}-01$ & 84.78 \\
\hline $6.300 \mathrm{E}-06$ & 0.002 & $7.20 \mathrm{E}-01$ & 69.10 \\
\hline $9.200 \mathrm{E}-06$ & 0.002 & $7.80 \mathrm{E}-01$ & 79.16 \\
\hline $1.350 \mathrm{E}-05$ & 0.001 & $8.40 \mathrm{E}-01$ & 76.97 \\
\hline $2.100 \mathrm{E}-05$ & 0.001 & $9.20 \mathrm{E}-01$ & 60.61 \\
\hline $3.000 E-05$ & 0.001 & $1.00 \mathrm{E}+00$ & 67.53 \\
\hline $4.500 \mathrm{E}-05$ & 0.001 & $1.20 \mathrm{E}+00$ & 88.97 \\
\hline $6.900 \mathrm{E}-05$ & 0.001 & $1.40 \mathrm{E}+00$ & 72.10 \\
\hline $1.000 \mathrm{E}-04$ & 0.001 & $1.60 \mathrm{E}+00$ & 110.78 \\
\hline $1.350 \mathrm{E}-04$ & 0.000 & $1.80 \mathrm{E}+00$ & 99.72 \\
\hline $1.700 \mathrm{E}-04$ & 0.029 & $2.00 \mathrm{E}+00$ & 105.97 \\
\hline $2.200 \mathrm{E}-04$ & 0.073 & $2.30 \mathrm{E}+00$ & 105.53 \\
\hline $2.800 \mathrm{E}-04$ & 0.107 & $2.60 \mathrm{E}+00$ & 127.16 \\
\hline $3.600 \mathrm{E}-04$ & 0.124 & $2.90 \mathrm{E}+00$ & 103.66 \\
\hline $4.500 \mathrm{E}-04$ & 0.140 & $3.30 \mathrm{E}+00$ & 118.41 \\
\hline $5.750 \mathrm{E}-04$ & 0.168 & $3.70 \mathrm{E}+00$ & 101.54 \\
\hline $7.600 \mathrm{E}-04$ & 0.206 & $4.10 \mathrm{E}+00$ & 134.67 \\
\hline $9.600 \mathrm{E}-04$ & 0.254 & $4.50 \mathrm{E}+00$ & 129.98 \\
\hline $1.275 \mathrm{E}-03$ & 0.317 & $5.00 \mathrm{E}+00$ & 124.61 \\
\hline $1.600 \mathrm{E}-03$ & 0.391 & $5.50 \mathrm{E}+00$ & 124.68 \\
\hline
\end{tabular}




$\begin{array}{lrrr}2.000 \mathrm{E}-03 & 0.506 & 6.00 \mathrm{E}+00 & 108.75 \\ 2.700 \mathrm{E}-03 & 0.655 & 6.70 \mathrm{E}+00 & 122.44 \\ 3.400 \mathrm{E}-03 & 0.839 & 7.40 \mathrm{E}+00 & 121.45 \\ 4.500 \mathrm{E}-03 & 1.053 & 8.20 \mathrm{E}+00 & 130.33 \\ 5.500 \mathrm{E}-03 & 1.326 & 9.00 \mathrm{E}+00 & 130.22 \\ 7.200 \mathrm{E}-03 & 1.695 & 1.00 \mathrm{E}+01 & 137.29 \\ 9.200 \mathrm{E}-03 & 2.180 & 1.10 \mathrm{E}+01 & 143.99 \\ 1.200 \mathrm{E}-02 & 2.737 & 1.20 \mathrm{E}+01 & 150.93 \\ 1.500 \mathrm{E}-02 & 3.926 & 1.30 \mathrm{E}+01 & 153.75 \\ 1.900 \mathrm{E}-02 & 7.041 & 1.40 \mathrm{E}+01 & 159.95 \\ 2.550 \mathrm{E}-02 & 6.288 & 1.50 \mathrm{E}+01 & 158.59 \\ 3.200 \mathrm{E}-02 & 7.388 & 1.60 \mathrm{E}+01 & 158.22 \\ 4.000 \mathrm{E}-02 & 9.213 & 1.70 \mathrm{E}+01 & 159.54 \\ 5.250 \mathrm{E}-02 & 12.432 & 1.80 \mathrm{E}+01 & 160.18 \\ 6.600 \mathrm{E}-02 & 92.949 & 1.90 \mathrm{E}+01 & 160.31 \\ & & 2.00 \mathrm{E}+01 & \end{array}$

\section{ALUMINIUM 1313}

\begin{tabular}{|c|c|c|c|}
\hline LOWER & CROSS & LOWER & CROSS \\
\hline $\begin{array}{l}\text { ENERGY } \\
(\mathrm{MEV})\end{array}$ & $\begin{array}{c}\text { SECTION } \\
(\mathrm{KEV}-\mathrm{B})\end{array}$ & $\begin{array}{l}\text { ENERGY } \\
(\mathrm{MEV})\end{array}$ & $\begin{array}{c}\text { SECTION } \\
(\mathrm{KEV}-\mathrm{B})\end{array}$ \\
\hline $1.000 \mathrm{E}-10$ & 1.195 & $8.80 \mathrm{E}-02$ & 35.15 \\
\hline $1.000 \mathrm{E}-09$ & 0.378 & $1.10 \mathrm{E}-01$ & 18.19 \\
\hline $1.000 \mathrm{E}-08$ & 0.198 & $1.35 \mathrm{E}-01$ & 61.45 \\
\hline $2.300 \mathrm{E}-08$ & 0.133 & $1.60 \mathrm{E}-01$ & 35.39 \\
\hline $5.000 \mathrm{E}-08$ & 0.100 & $1.90 \mathrm{E}-01$ & 42.91 \\
\hline $7.600 \mathrm{E}-08$ & 0.081 & $2.20 \mathrm{E}-01$ & 31.52 \\
\hline $1.150 \mathrm{E}-07$ & 0.066 & $2.55 E-01$ & 34.40 \\
\hline $1.700 \mathrm{E}-07$ & 0.054 & $2.90 \mathrm{E}-01$ & 57.59 \\
\hline $2.550 \mathrm{E}-07$ & 0.044 & $3.20 \mathrm{E}-01$ & 36.47 \\
\hline $3.800 \mathrm{E}-07$ & 0.037 & $3.60 \mathrm{E}-01$ & 51.76 \\
\hline $5.500 \mathrm{E}-07$ & 0.030 & $4.00 \mathrm{E}-01$ & 68.19 \\
\hline $8.400 E-07$ & 0.024 & $4.50 \mathrm{E}-01$ & 63.65 \\
\hline $1.275 E-06$ & 0.020 & $5.00 \mathrm{E}-01$ & 68.52 \\
\hline $1.900 \mathrm{E}-06$ & 0.016 & $5.50 \mathrm{E}-01$ & 70.95 \\
\hline $2.800 \mathrm{E}-06$ & 0.013 & $6.00 \mathrm{E}-01$ & 74.12 \\
\hline $4.250 \mathrm{E}-06$ & 0.011 & $6.60 \mathrm{E}-01$ & 63.56 \\
\hline $6.300 \mathrm{E}-06$ & 0.009 & $7.20 \mathrm{E}-01$ & 80.87 \\
\hline $9.200 \mathrm{E}-06$ & 0.007 & $7.80 \mathrm{E}-01$ & 99.23 \\
\hline $1.350 \mathrm{E}-05$ & 0.006 & $8.40 \mathrm{E}-01$ & 83.64 \\
\hline $2.100 \mathrm{E}-05$ & 0.005 & $9.20 \mathrm{E}-01$ & 70.27 \\
\hline $3.000 E-05$ & 0.004 & $1.00 \mathrm{E}+00$ & 87.82 \\
\hline $4.500 \mathrm{E}-05$ & 0.003 & $1.20 \mathrm{E}+00$ & 86.54 \\
\hline $6.900 \mathrm{E}-05$ & 0.003 & $1.40 \mathrm{E}+00$ & 100.11 \\
\hline $1.000 \mathrm{E}-04$ & 0.002 & $1.60 \mathrm{E}+00$ & 103.96 \\
\hline $1.350 \mathrm{E}-04$ & 0.002 & $1.80 \mathrm{E}+00$ & 104.50 \\
\hline $1.700 \mathrm{E}-04$ & 0.002 & $2.00 \mathrm{E}+00$ & 122.32 \\
\hline $2.200 \mathrm{E}-04$ & 0.020 & $2.30 \mathrm{E}+00$ & 114.42 \\
\hline $2.800 E-04$ & 0.035 & $2.60 \mathrm{E}+00$ & 118.27 \\
\hline $3.600 \mathrm{E}-04$ & 0.047 & $2.90 \mathrm{E}+00$ & 122.86 \\
\hline $4.500 E-04$ & 0.052 & $3.30 \mathrm{E}+00$ & 125.76 \\
\hline $5.750 \mathrm{E}-04$ & 0.061 & $3.70 \mathrm{E}+00$ & 128.93 \\
\hline $7.600 \mathrm{E}-04$ & 0.074 & $4.10 \mathrm{E}+00$ & 126.71 \\
\hline $9.600 \mathrm{E}-04$ & 0.091 & $4.50 \mathrm{E}+00$ & 129.27 \\
\hline $1.275 E-03$ & 0.114 & $5.00 \mathrm{E}+00$ & 134.94 \\
\hline
\end{tabular}




$\begin{array}{lrrr}1.600 \mathrm{E}-03 & 0.140 & 5.50 \mathrm{E}+00 & 136.77 \\ 2.000 \mathrm{E}-03 & 0.180 & 6.00 \mathrm{E}+00 & 139.54 \\ 2.700 \mathrm{E}-03 & 0.230 & 6.70 \mathrm{E}+00 & 139.55 \\ 3.400 \mathrm{E}-03 & 0.290 & 7.40 \mathrm{E}+00 & 143.34 \\ 4.500 \mathrm{E}-03 & 0.369 & 8.20 \mathrm{E}+00 & 144.17 \\ 5.500 \mathrm{E}-03 & 0.877 & 9.00 \mathrm{E}+00 & 145.81 \\ 7.200 \mathrm{E}-03 & 0.579 & 1.00 \mathrm{E}+01 & 151.97 \\ 9.200 \mathrm{E}-03 & 0.705 & 1.10 \mathrm{E}+01 & 160.57 \\ 1.200 \mathrm{E}-02 & 0.841 & 1.20 \mathrm{E}+01 & 168.63 \\ 1.500 \mathrm{E}-02 & 0.821 & 1.30 \mathrm{E}+01 & 172.71 \\ 1.900 \mathrm{E}-02 & 0.801 & 1.40 \mathrm{E}+01 & 177.07 \\ 2.550 \mathrm{E}-02 & 2.263 & 1.50 \mathrm{E}+01 & 180.42 \\ 3.200 \mathrm{E}-02 & 32.802 & 1.60 \mathrm{E}+01 & 181.77 \\ 4.000 \mathrm{E}-02 & 8.200 & 1.70 \mathrm{E}+01 & 182.03 \\ 5.250 \mathrm{E}-02 & 5.273 & 1.80 \mathrm{E}+01 & 181.23 \\ 6.600 \mathrm{E}-02 & 24.454 & 1.90 \mathrm{E}+01 & 179.89 \\ & & 2.00 \mathrm{E}+01 & \end{array}$

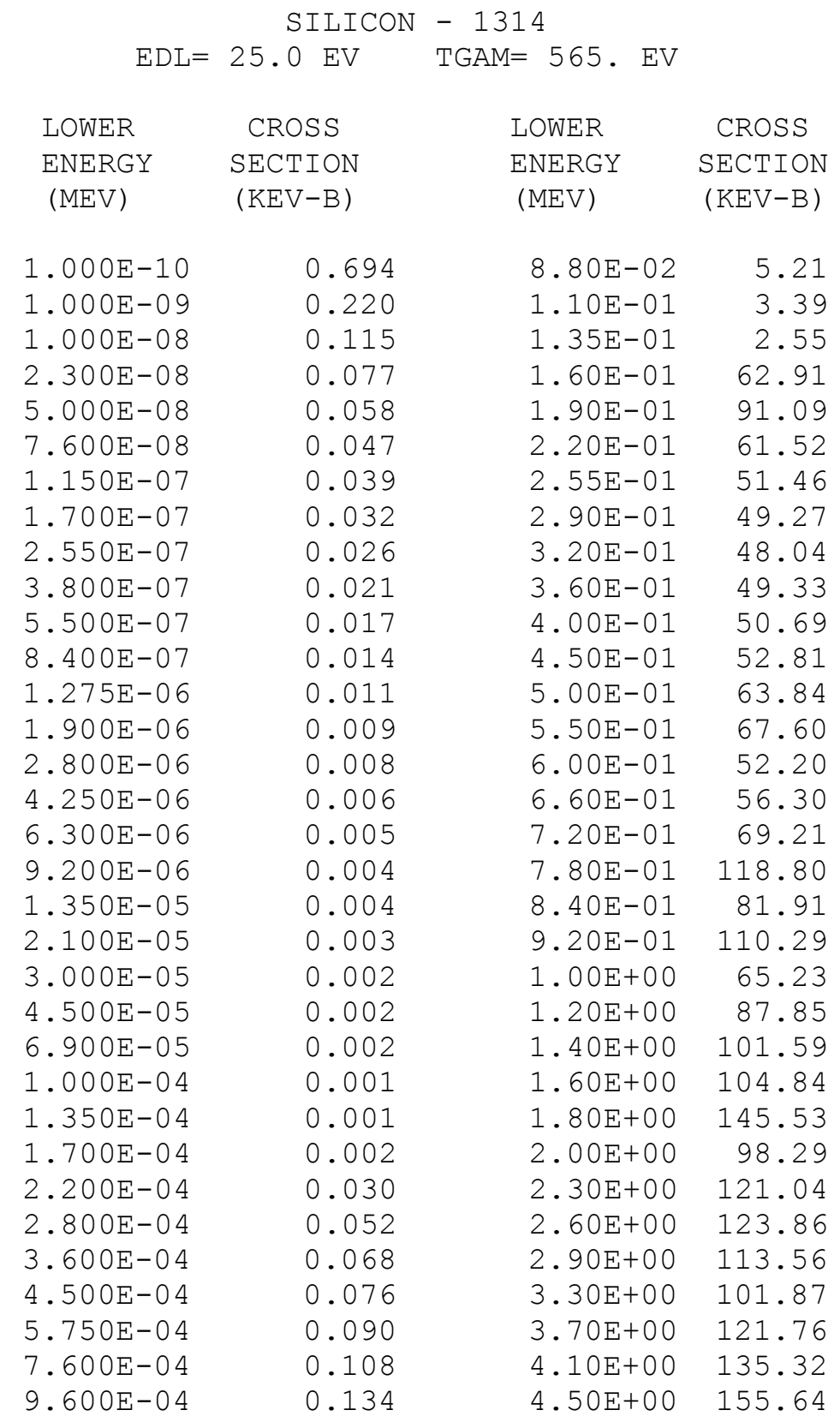




$\begin{array}{lrrr}1.275 \mathrm{E}-03 & 0.168 & 5.00 \mathrm{E}+00 & 138.03 \\ 1.600 \mathrm{E}-03 & 0.207 & 5.50 \mathrm{E}+00 & 147.78 \\ 2.000 \mathrm{E}-03 & 0.264 & 6.00 \mathrm{E}+00 & 134.67 \\ 2.700 \mathrm{E}-03 & 0.337 & 6.70 \mathrm{E}+00 & 131.43 \\ 3.400 \mathrm{E}-03 & 0.430 & 7.40 \mathrm{E}+00 & 169.06 \\ 4.500 \mathrm{E}-03 & 0.562 & 8.20 \mathrm{E}+00 & 165.45 \\ 5.500 \mathrm{E}-03 & 0.683 & 9.00 \mathrm{E}+00 & 168.46 \\ 7.200 \mathrm{E}-03 & 0.857 & 1.00 \mathrm{E}+01 & 171.66 \\ 9.200 \mathrm{E}-03 & 1.071 & 1.10 \mathrm{E}+01 & 176.48 \\ 1.200 \mathrm{E}-02 & 1.330 & 1.20 \mathrm{E}+01 & 181.37 \\ 1.500 \mathrm{E}-02 & 1.638 & 1.30 \mathrm{E}+01 & 191.19 \\ 1.900 \mathrm{E}-02 & 2.027 & 1.40 \mathrm{E}+01 & 191.57 \\ 2.550 \mathrm{E}-02 & 2.452 & 1.50 \mathrm{E}+01 & 194.15 \\ 3.200 \mathrm{E}-02 & 3.004 & 1.60 \mathrm{E}+01 & 196.03 \\ 4.000 \mathrm{E}-02 & 2.632 & 1.70 \mathrm{E}+01 & 195.91 \\ 5.250 \mathrm{E}-02 & 14.335 & 1.80 \mathrm{E}+01 & 199.86 \\ 6.600 \mathrm{E}-02 & 6.313 & 1.90 \mathrm{E}+01 & 200.24 \\ & & 2.00 \mathrm{E}+01 & \end{array}$

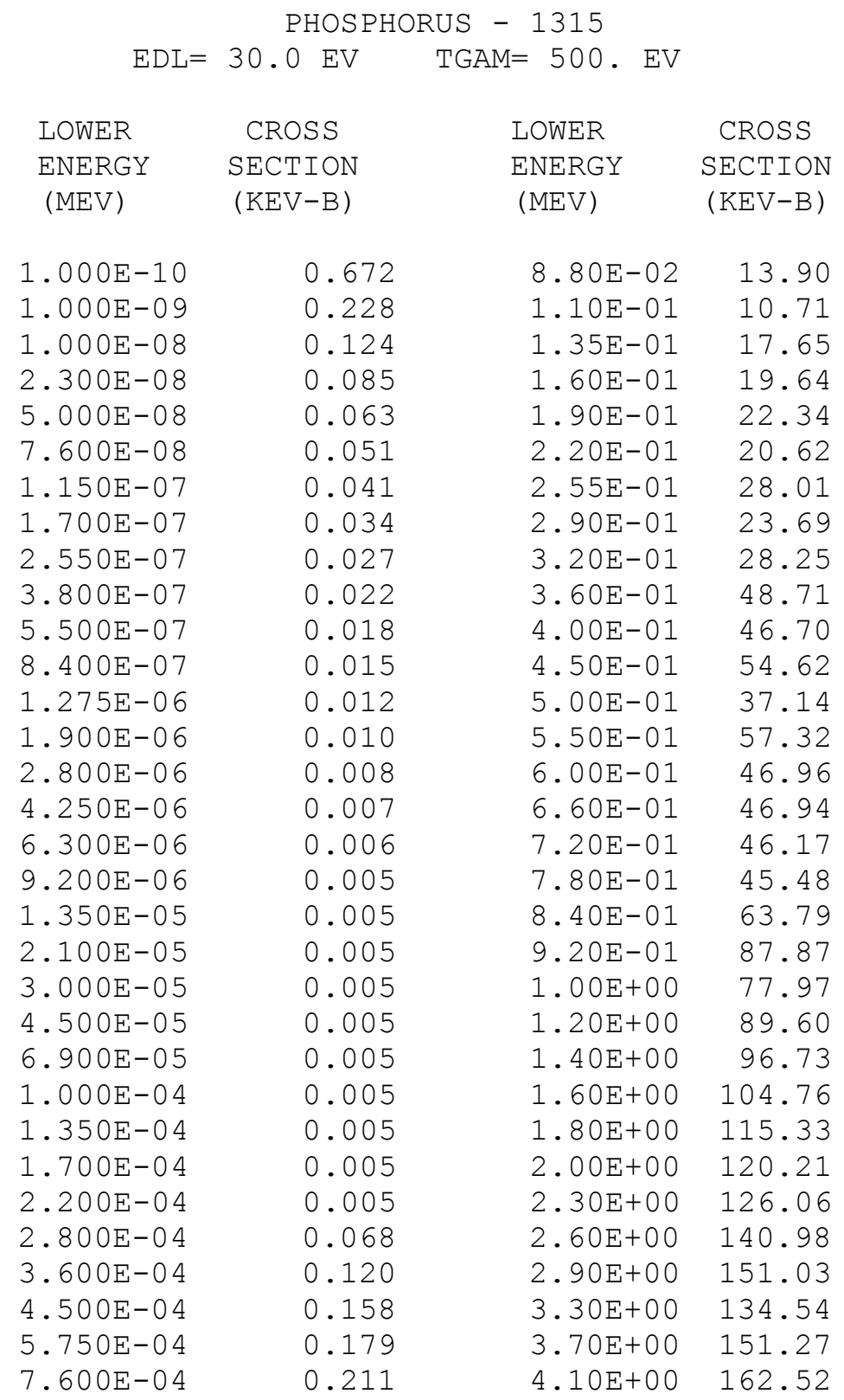




$\begin{array}{lrrr}9.600 \mathrm{E}-04 & 0.258 & 4.50 \mathrm{E}+00 & 149.33 \\ 1.275 \mathrm{E}-03 & 0.317 & 5.00 \mathrm{E}+00 & 150.08 \\ 1.600 \mathrm{E}-03 & 0.384 & 5.50 \mathrm{E}+00 & 141.84 \\ 2.000 \mathrm{E}-03 & 0.492 & 6.00 \mathrm{E}+00 & 155.87 \\ 2.700 \mathrm{E}-03 & 0.626 & 6.70 \mathrm{E}+00 & 166.22 \\ 3.400 \mathrm{E}-03 & 0.799 & 7.40 \mathrm{E}+00 & 165.25 \\ 4.500 \mathrm{E}-03 & 1.000 & 8.20 \mathrm{E}+00 & 168.18 \\ 5.500 \mathrm{E}-03 & 1.327 & 9.00 \mathrm{E}+00 & 170.36 \\ 7.200 \mathrm{E}-03 & 2.036 & 1.00 \mathrm{E}+01 & 169.99 \\ 9.200 \mathrm{E}-03 & 2.460 & 1.10 \mathrm{E}+01 & 172.39 \\ 1.200 \mathrm{E}-02 & 3.189 & 1.20 \mathrm{E}+01 & 177.64 \\ 1.500 \mathrm{E}-02 & 3.429 & 1.30 \mathrm{E}+01 & 186.93 \\ 1.900 \mathrm{E}-02 & 3.844 & 1.40 \mathrm{E}+01 & 186.63 \\ 2.550 \mathrm{E}-02 & 5.246 & 1.50 \mathrm{E}+01 & 192.86 \\ 3.200 \mathrm{E}-02 & 7.198 & 1.60 \mathrm{E}+01 & 192.03 \\ 4.000 \mathrm{E}-02 & 9.431 & 1.70 \mathrm{E}+01 & 192.11 \\ 5.250 \mathrm{E}-02 & 11.435 & 1.80 \mathrm{E}+01 & 190.76 \\ 6.600 \mathrm{E}-02 & 14.190 & 1.90 \mathrm{E}+01 & 192.79 \\ & & 2.00 \mathrm{E}+01 & \end{array}$

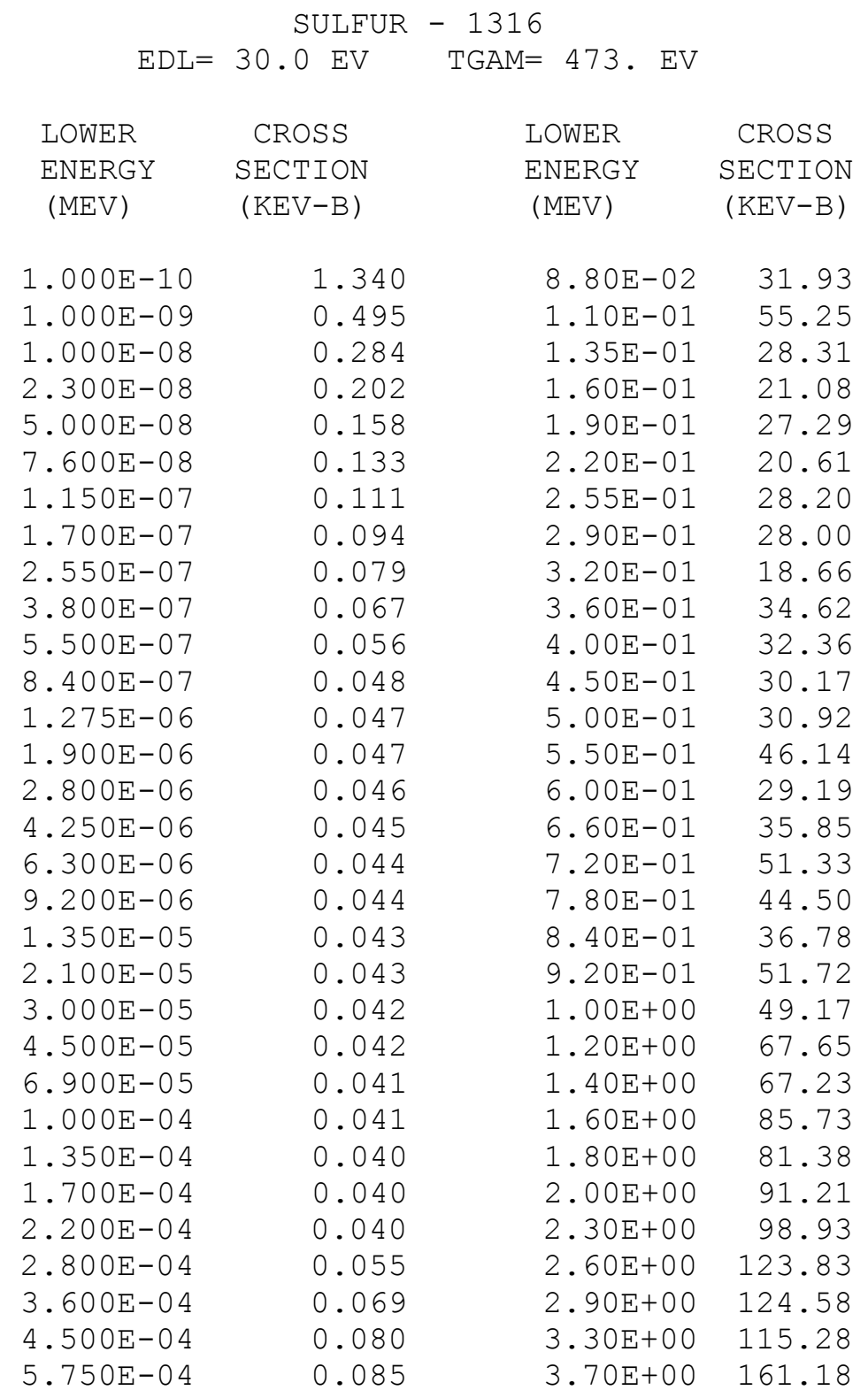




$\begin{array}{llll}7.600 \mathrm{E}-04 & 0.092 & 4.10 \mathrm{E}+00 & 158.48 \\ 9.600 \mathrm{E}-04 & 0.101 & 4.50 \mathrm{E}+00 & 153.01 \\ 1.275 \mathrm{E}-03 & 0.111 & 5.00 \mathrm{E}+00 & 146.34 \\ 1.600 \mathrm{E}-03 & 0.125 & 5.50 \mathrm{E}+00 & 160.89 \\ 2.000 \mathrm{E}-03 & 0.147 & 6.00 \mathrm{E}+00 & 183.91 \\ 2.700 \mathrm{E}-03 & 0.176 & 6.70 \mathrm{E}+00 & 178.21 \\ 3.400 \mathrm{E}-03 & 0.214 & 7.40 \mathrm{E}+00 & 178.21 \\ 4.500 \mathrm{E}-03 & 0.258 & 8.20 \mathrm{E}+00 & 186.46 \\ 5.500 \mathrm{E}-03 & 0.324 & 9.00 \mathrm{E}+00 & 189.54 \\ 7.200 \mathrm{E}-03 & 0.423 & 1.00 \mathrm{E}+01 & 193.96 \\ 9.200 \mathrm{E}-03 & 0.574 & 1.10 \mathrm{E}+01 & 195.84 \\ 1.200 \mathrm{E}-02 & 0.760 & 1.20 \mathrm{E}+01 & 194.94 \\ 1.500 \mathrm{E}-02 & 0.794 & 1.30 \mathrm{E}+01 & 190.89 \\ 1.900 \mathrm{E}-02 & 0.797 & 1.40 \mathrm{E}+01 & 192.99 \\ 2.550 \mathrm{E}-02 & 1.209 & 1.50 \mathrm{E}+01 & 191.19 \\ 3.200 \mathrm{E}-02 & 0.972 & 1.60 \mathrm{E}+01 & 207.91 \\ 4.000 \mathrm{E}-02 & 1.095 & 1.70 \mathrm{E}+01 & 212.11 \\ 5.250 \mathrm{E}-02 & 1.241 & 1.80 \mathrm{E}+01 & 216.08 \\ 6.600 \mathrm{E}-02 & 4.500 & 1.90 \mathrm{E}+01 & 216.61 \\ & & 2.00 \mathrm{E}+01 & \end{array}$

CHLORINE 1149

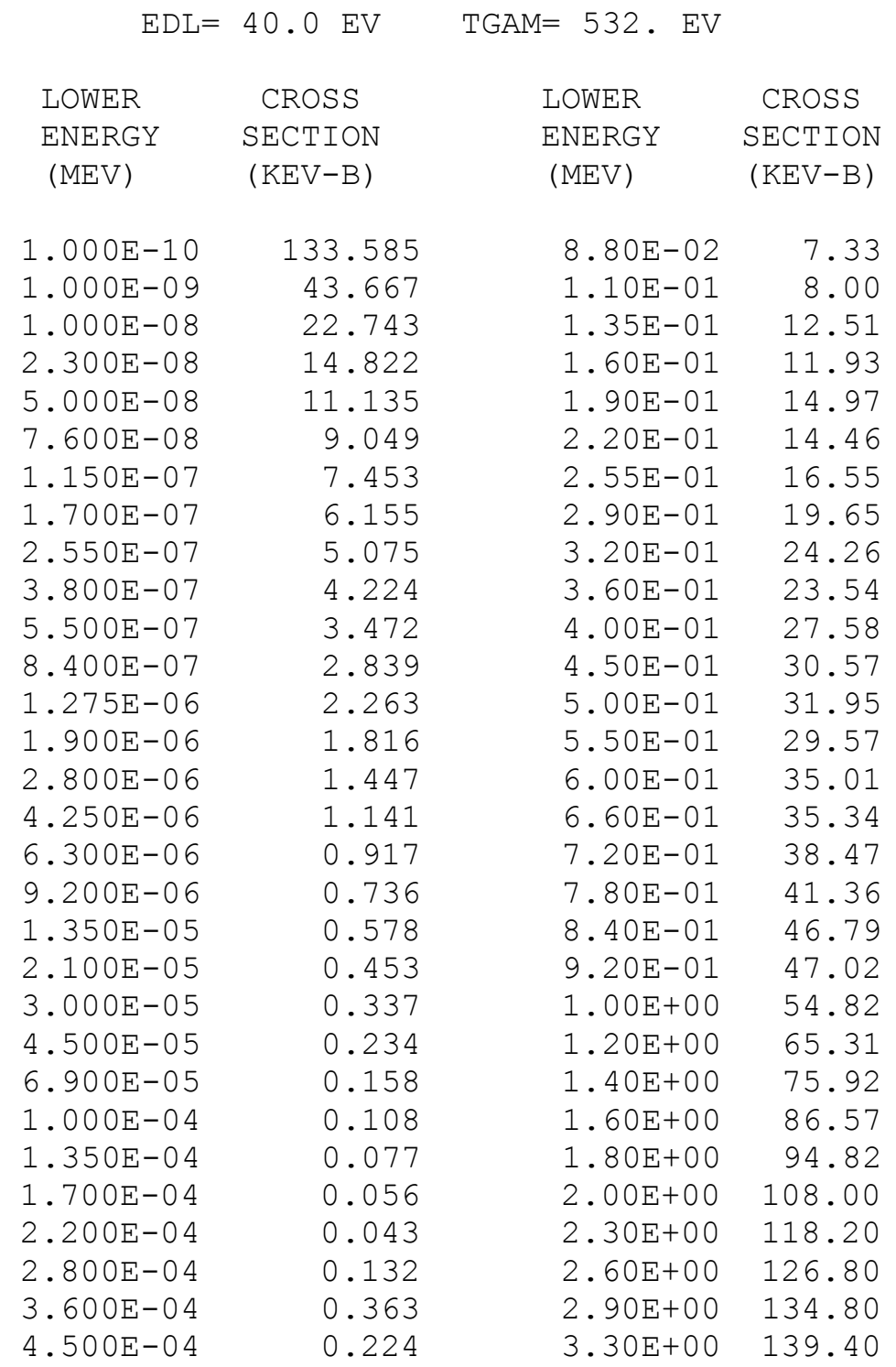




$\begin{array}{llll}5.750 \mathrm{E}-04 & 0.141 & 3.70 \mathrm{E}+00 & 139.10 \\ 7.600 \mathrm{E}-04 & 0.136 & 4.10 \mathrm{E}+00 & 141.80 \\ 9.600 \mathrm{E}-04 & 0.137 & 4.50 \mathrm{E}+00 & 146.90 \\ 1.275 \mathrm{E}-03 & 0.144 & 5.00 \mathrm{E}+00 & 147.80 \\ 1.600 \mathrm{E}-03 & 0.166 & 5.50 \mathrm{E}+00 & 153.60 \\ 2.000 \mathrm{E}-03 & 0.172 & 6.00 \mathrm{E}+00 & 173.40 \\ 2.700 \mathrm{E}-03 & 0.246 & 6.70 \mathrm{E}+00 & 176.70 \\ 3.400 \mathrm{E}-03 & 0.365 & 7.40 \mathrm{E}+00 & 188.00 \\ 4.500 \mathrm{E}-03 & 0.307 & 8.20 \mathrm{E}+00 & 194.70 \\ 5.500 \mathrm{E}-03 & 0.541 & 9.00 \mathrm{E}+00 & 200.70 \\ 7.200 \mathrm{E}-03 & 1.605 & 1.00 \mathrm{E}+01 & 208.40 \\ 9.200 \mathrm{E}-03 & 0.908 & 1.10 \mathrm{E}+01 & 219.20 \\ 1.200 \mathrm{E}-02 & 2.042 & 1.20 \mathrm{E}+01 & 229.20 \\ 1.500 \mathrm{E}-02 & 2.271 & 1.30 \mathrm{E}+01 & 237.50 \\ 1.900 \mathrm{E}-02 & 3.533 & 1.40 \mathrm{E}+01 & 243.30 \\ 2.550 \mathrm{E}-02 & 5.020 & 1.50 \mathrm{E}+01 & 244.70 \\ 3.200 \mathrm{E}-02 & 3.289 & 1.60 \mathrm{E}+01 & 244.90 \\ 4.000 \mathrm{E}-02 & 4.858 & 1.70 \mathrm{E}+01 & 247.70 \\ 5.250 \mathrm{E}-02 & 6.001 & 1.80 \mathrm{E}+01 & 249.80 \\ 6.600 \mathrm{E}-02 & 7.024 & 1.90 \mathrm{E}+01 & 258.70 \\ & & 2.00 \mathrm{E}+01 & \end{array}$

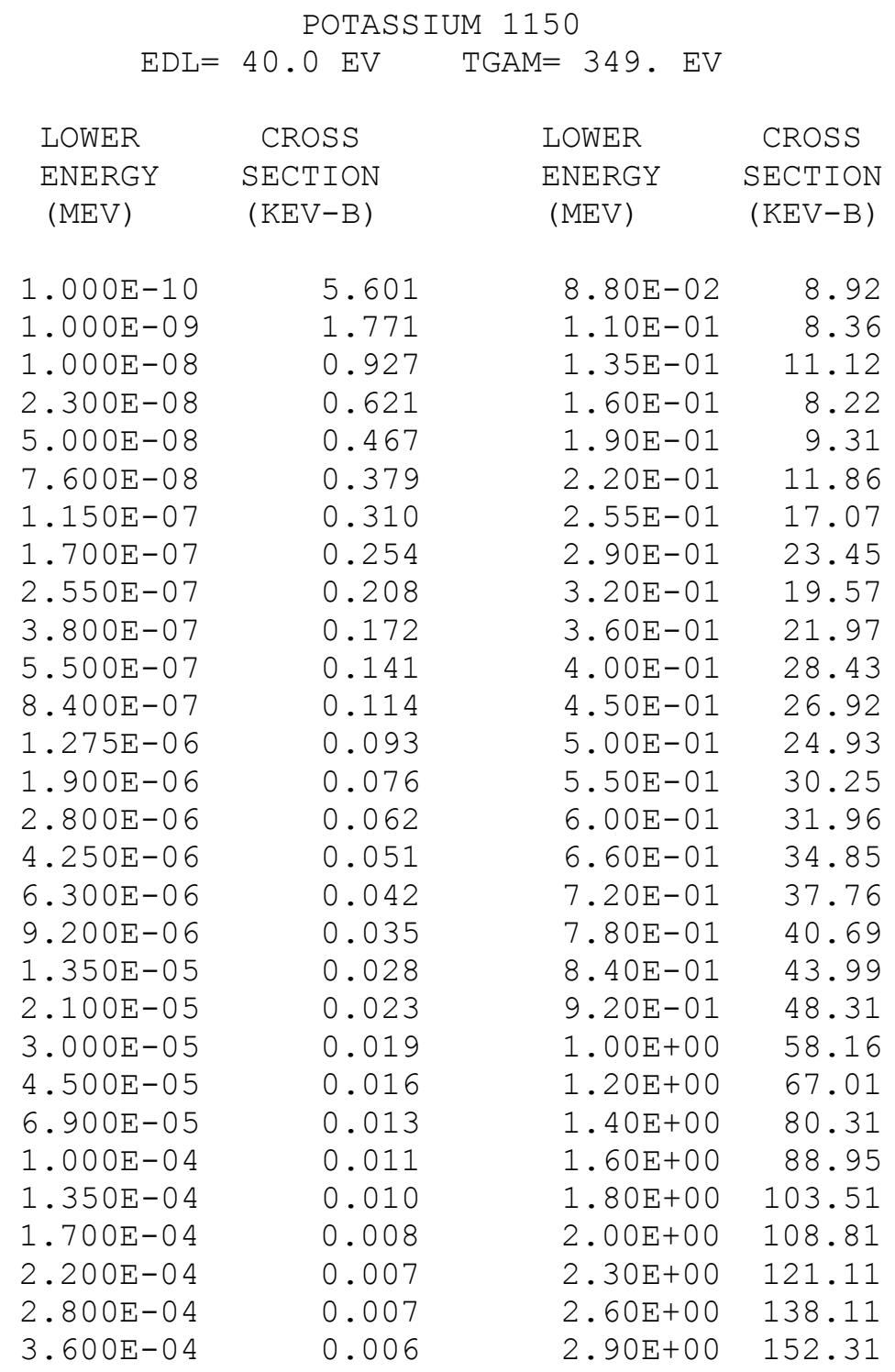




$\begin{array}{llll}4.500 \mathrm{E}-04 & 0.031 & 3.30 \mathrm{E}+00 & 157.61 \\ 5.750 \mathrm{E}-04 & 0.054 & 3.70 \mathrm{E}+00 & 161.01 \\ 7.600 \mathrm{E}-04 & 0.069 & 4.10 \mathrm{E}+00 & 160.41 \\ 9.600 \mathrm{E}-04 & 0.075 & 4.50 \mathrm{E}+00 & 156.11 \\ 1.275 \mathrm{E}-03 & 0.083 & 5.00 \mathrm{E}+00 & 185.11 \\ 1.600 \mathrm{E}-03 & 0.094 & 5.50 \mathrm{E}+00 & 192.01 \\ 2.000 \mathrm{E}-03 & 0.113 & 6.00 \mathrm{E}+00 & 211.41 \\ 2.700 \mathrm{E}-03 & 1.258 & 6.70 \mathrm{E}+00 & 214.61 \\ 3.400 \mathrm{E}-03 & 1.769 & 7.40 \mathrm{E}+00 & 227.61 \\ 4.500 \mathrm{E}-03 & 0.472 & 8.20 \mathrm{E}+00 & 233.11 \\ 5.500 \mathrm{E}-03 & 0.351 & 9.00 \mathrm{E}+00 & 239.11 \\ 7.200 \mathrm{E}-03 & 1.110 & 1.00 \mathrm{E}+01 & 245.51 \\ 9.200 \mathrm{E}-03 & 2.083 & 1.10 \mathrm{E}+01 & 248.11 \\ 1.200 \mathrm{E}-02 & 0.659 & 1.20 \mathrm{E}+01 & 255.11 \\ 1.500 \mathrm{E}-02 & 1.356 & 1.30 \mathrm{E}+01 & 254.51 \\ 1.900 \mathrm{E}-02 & 1.591 & 1.40 \mathrm{E}+01 & 256.71 \\ 2.550 \mathrm{E}-02 & 2.898 & 1.50 \mathrm{E}+01 & 258.81 \\ 3.200 \mathrm{E}-02 & 2.797 & 1.60 \mathrm{E}+01 & 261.61 \\ 4.000 \mathrm{E}-02 & 4.827 & 1.70 \mathrm{E}+01 & 266.21 \\ 5.250 \mathrm{E}-02 & 7.159 & 1.80 \mathrm{E}+01 & 271.81 \\ 6.600 \mathrm{E}-02 & 7.795 & 1.90 \mathrm{E}+01 & 275.51 \\ & & 2.00 \mathrm{E}+01 & \end{array}$

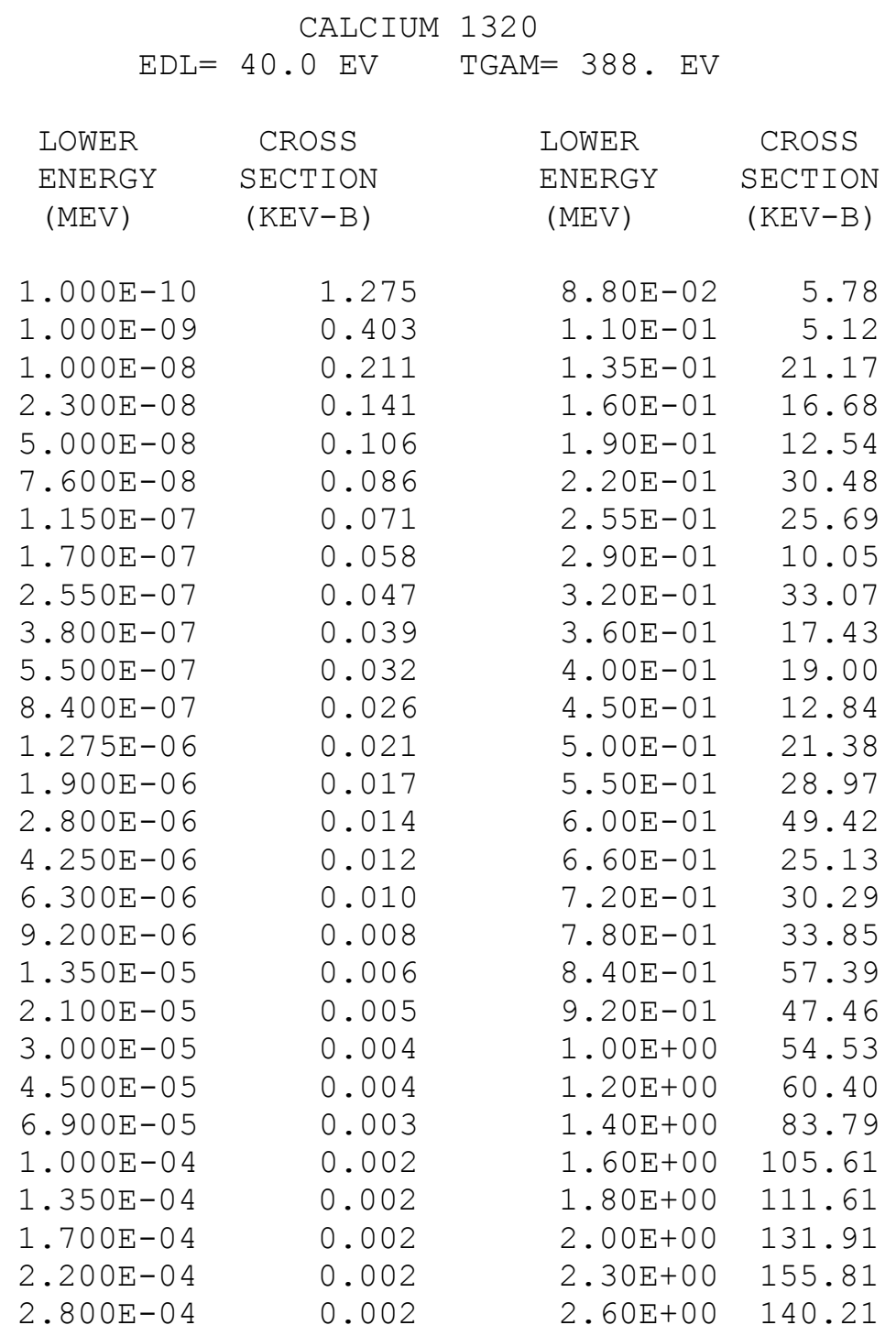




$\begin{array}{llll}3.600 \mathrm{E}-04 & 0.001 & 2.90 \mathrm{E}+00 & 155.41 \\ 4.500 \mathrm{E}-04 & 0.051 & 3.30 \mathrm{E}+00 & 147.41 \\ 5.750 \mathrm{E}-04 & 0.108 & 3.70 \mathrm{E}+00 & 174.91 \\ 7.600 \mathrm{E}-04 & 0.152 & 4.10 \mathrm{E}+00 & 172.01 \\ 9.600 \mathrm{E}-04 & 0.170 & 4.50 \mathrm{E}+00 & 167.01 \\ 1.275 \mathrm{E}-03 & 0.196 & 5.00 \mathrm{E}+00 & 214.61 \\ 1.600 \mathrm{E}-03 & 0.224 & 5.50 \mathrm{E}+00 & 217.51 \\ 2.000 \mathrm{E}-03 & 0.266 & 6.00 \mathrm{E}+00 & 219.02 \\ 2.700 \mathrm{E}-03 & 0.321 & 6.70 \mathrm{E}+00 & 224.22 \\ 3.400 \mathrm{E}-03 & 0.389 & 7.40 \mathrm{E}+00 & 228.23 \\ 4.500 \mathrm{E}-03 & 0.462 & 8.20 \mathrm{E}+00 & 257.04 \\ 5.500 \mathrm{E}-03 & 0.549 & 9.00 \mathrm{E}+00 & 267.65 \\ 7.200 \mathrm{E}-03 & 0.661 & 1.00 \mathrm{E}+01 & 272.07 \\ 9.200 \mathrm{E}-03 & 0.855 & 1.10 \mathrm{E}+01 & 275.79 \\ 1.200 \mathrm{E}-02 & 1.025 & 1.20 \mathrm{E}+01 & 279.41 \\ 1.500 \mathrm{E}-02 & 1.258 & 1.30 \mathrm{E}+01 & 278.13 \\ 1.900 \mathrm{E}-02 & 1.609 & 1.40 \mathrm{E}+01 & 273.25 \\ 2.550 \mathrm{E}-02 & 1.616 & 1.50 \mathrm{E}+01 & 271.96 \\ 3.200 \mathrm{E}-02 & 1.883 & 1.60 \mathrm{E}+01 & 260.37 \\ 4.000 \mathrm{E}-02 & 2.327 & 1.70 \mathrm{E}+01 & 259.58 \\ 5.250 \mathrm{E}-02 & 2.981 & 1.80 \mathrm{E}+01 & 259.29 \\ 6.600 \mathrm{E}-02 & 3.802 & 1.90 \mathrm{E}+01 & 258.00 \\ & & 2.00 \mathrm{E}+01 & \end{array}$

\begin{tabular}{|c|c|c|c|}
\hline \multicolumn{4}{|c|}{ TITANIUM 1322} \\
\hline EDL & $40.0 \mathrm{EV}$ & $M=400 . E V$ & \\
\hline LOWER & CROSS & LOWER & CROSS \\
\hline ENERGY & SECTION & ENERGY & SECTION \\
\hline$(\mathrm{MEV})$ & $(\mathrm{KEV}-\mathrm{B})$ & $(\mathrm{MEV})$ & $(\mathrm{KEV}-\mathrm{B})$ \\
\hline $1.000 \mathrm{E}-10$ & 18.704 & $8.80 E-02$ & 9.41 \\
\hline $1.000 \mathrm{E}-09$ & 5.912 & $1.10 \mathrm{E}-01$ & 7.82 \\
\hline $1.000 \mathrm{E}-08$ & 3.092 & $1.35 \mathrm{E}-01$ & 7.30 \\
\hline $2.300 \mathrm{E}-08$ & 2.072 & $1.60 \mathrm{E}-01$ & 6.62 \\
\hline $5.000 \mathrm{E}-08$ & 1.556 & $1.90 \mathrm{E}-01$ & 16.07 \\
\hline $7.600 \mathrm{E}-08$ & 1.262 & $2.20 \mathrm{E}-01$ & 13.76 \\
\hline $1.150 \mathrm{E}-07$ & 1.032 & $2.55 \mathrm{E}-01$ & 21.09 \\
\hline $1.700 \mathrm{E}-07$ & 0.843 & $2.90 \mathrm{E}-01$ & 15.16 \\
\hline $2.550 \mathrm{E}-07$ & 0.688 & $3.20 \mathrm{E}-01$ & 19.19 \\
\hline $3.800 \mathrm{E}-07$ & 0.565 & $3.60 \mathrm{E}-01$ & 13.41 \\
\hline $5.500 \mathrm{E}-07$ & 0.460 & $4.00 \mathrm{E}-01$ & 22.22 \\
\hline $8.400 \mathrm{E}-07$ & 0.369 & $4.50 \mathrm{E}-01$ & 35.46 \\
\hline $1.275 E-06$ & 0.296 & $5.00 \mathrm{E}-01$ & 24.89 \\
\hline $1.900 \mathrm{E}-06$ & 0.238 & $5.50 \mathrm{E}-01$ & 30.71 \\
\hline $2.800 \mathrm{E}-06$ & 0.189 & $6.00 \mathrm{E}-01$ & 34.90 \\
\hline $4.250 \mathrm{E}-06$ & 0.146 & $6.60 \mathrm{E}-01$ & 56.21 \\
\hline $6.300 \mathrm{E}-06$ & 0.114 & $7.20 \mathrm{E}-01$ & 55.29 \\
\hline $9.200 \mathrm{E}-06$ & 0.087 & $7.80 \mathrm{E}-01$ & 41.42 \\
\hline $1.350 \mathrm{E}-05$ & 0.063 & $8.40 \mathrm{E}-01$ & 50.06 \\
\hline $2.100 \mathrm{E}-05$ & 0.046 & $9.20 \mathrm{E}-01$ & 58.55 \\
\hline $3.000 \mathrm{E}-05$ & 0.033 & $1.00 \mathrm{E}+00$ & 48.98 \\
\hline $4.500 \mathrm{E}-05$ & 0.022 & $1.20 \mathrm{E}+00$ & 72.74 \\
\hline $6.900 \mathrm{E}-05$ & 0.016 & $1.40 \mathrm{E}+00$ & 88.17 \\
\hline $1.000 \mathrm{E}-04$ & 0.012 & $1.60 \mathrm{E}+00$ & 89.11 \\
\hline $1.350 \mathrm{E}-04$ & 0.010 & $1.80 \mathrm{E}+00$ & 119.65 \\
\hline $1.700 \mathrm{E}-04$ & 0.009 & $2.00 \mathrm{E}+00$ & 121.76 \\
\hline $2.200 \mathrm{E}-04$ & 0.007 & $2.30 \mathrm{E}+00$ & 139.96 \\
\hline
\end{tabular}




$\begin{array}{lrrr}2.800 \mathrm{E}-04 & 0.006 & 2.60 \mathrm{E}+00 & 139.56 \\ 3.600 \mathrm{E}-04 & 0.006 & 2.90 \mathrm{E}+00 & 146.57 \\ 4.500 \mathrm{E}-04 & 0.012 & 3.30 \mathrm{E}+00 & 157.56 \\ 5.750 \mathrm{E}-04 & 0.117 & 3.70 \mathrm{E}+00 & 162.26 \\ 7.600 \mathrm{E}-04 & 0.199 & 4.10 \mathrm{E}+00 & 172.26 \\ 9.600 \mathrm{E}-04 & 0.257 & 4.50 \mathrm{E}+00 & 171.45 \\ 1.275 \mathrm{E}-03 & 0.309 & 5.00 \mathrm{E}+00 & 173.24 \\ 1.600 \mathrm{E}-03 & 0.379 & 5.50 \mathrm{E}+00 & 176.34 \\ 2.000 \mathrm{E}-03 & 0.522 & 6.00 \mathrm{E}+00 & 181.93 \\ 2.700 \mathrm{E}-03 & 1.554 & 6.70 \mathrm{E}+00 & 186.83 \\ 3.400 \mathrm{E}-03 & 1.605 & 7.40 \mathrm{E}+00 & 192.13 \\ 4.500 \mathrm{E}-03 & 1.565 & 8.20 \mathrm{E}+00 & 197.13 \\ 5.500 \mathrm{E}-03 & 2.481 & 9.00 \mathrm{E}+00 & 204.03 \\ 7.200 \mathrm{E}-03 & 5.253 & 1.00 \mathrm{E}+01 & 208.63 \\ 9.200 \mathrm{E}-03 & 10.488 & 1.10 \mathrm{E}+01 & 209.83 \\ 1.200 \mathrm{E}-02 & 25.897 & 1.20 \mathrm{E}+01 & 218.26 \\ 1.500 \mathrm{E}-02 & 56.927 & 1.30 \mathrm{E}+01 & 231.22 \\ 1.900 \mathrm{E}-02 & 42.200 & 1.40 \mathrm{E}+01 & 244.00 \\ 2.550 \mathrm{E}-02 & 18.658 & 1.50 \mathrm{E}+01 & 255.83 \\ 3.200 \mathrm{E}-02 & 19.273 & 1.60 \mathrm{E}+01 & 268.37 \\ 4.000 \mathrm{E}-02 & 15.137 & 1.70 \mathrm{E}+01 & 279.30 \\ 5.250 \mathrm{E}-02 & 22.541 & 1.80 \mathrm{E}+01 & 289.53 \\ 6.600 \mathrm{E}-02 & 11.609 & 1.90 \mathrm{E}+01 & 298.68 \\ & & 2.00 \mathrm{E}+01 & \end{array}$

VANADIUM 1323

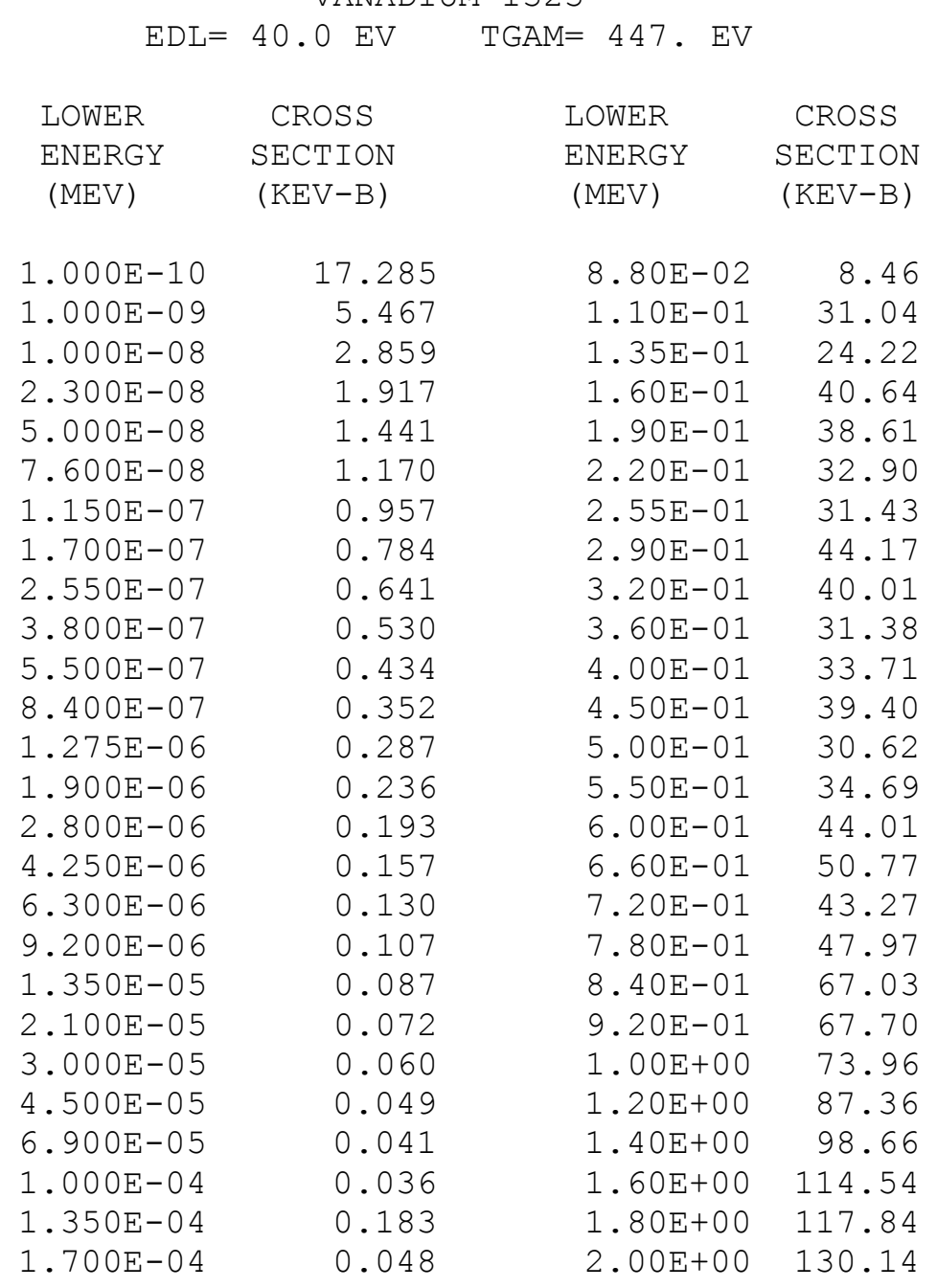




$\begin{array}{lrrr}2.200 \mathrm{E}-04 & 0.024 & 2.30 \mathrm{E}+00 & 134.04 \\ 2.800 \mathrm{E}-04 & 0.021 & 2.60 \mathrm{E}+00 & 145.64 \\ 3.600 \mathrm{E}-04 & 0.019 & 2.90 \mathrm{E}+00 & 150.05 \\ 4.500 \mathrm{E}-04 & 0.017 & 3.30 \mathrm{E}+00 & 159.25 \\ 5.750 \mathrm{E}-04 & 0.137 & 3.70 \mathrm{E}+00 & 161.25 \\ 7.600 \mathrm{E}-04 & 0.255 & 4.10 \mathrm{E}+00 & 165.36 \\ 9.600 \mathrm{E}-04 & 0.379 & 4.50 \mathrm{E}+00 & 169.76 \\ 1.275 \mathrm{E}-03 & 0.518 & 5.00 \mathrm{E}+00 & 171.47 \\ 1.600 \mathrm{E}-03 & 0.570 & 5.50 \mathrm{E}+00 & 177.57 \\ 2.000 \mathrm{E}-03 & 0.833 & 6.00 \mathrm{E}+00 & 186.57 \\ 2.700 \mathrm{E}-03 & 1.929 & 6.70 \mathrm{E}+00 & 192.28 \\ 3.400 \mathrm{E}-03 & 23.352 & 7.40 \mathrm{E}+00 & 196.68 \\ 4.500 \mathrm{E}-03 & 15.982 & 8.20 \mathrm{E}+00 & 205.98 \\ 5.500 \mathrm{E}-03 & 24.973 & 9.00 \mathrm{E}+00 & 216.28 \\ 7.200 \mathrm{E}-03 & 7.428 & 1.00 \mathrm{E}+01 & 225.68 \\ 9.200 \mathrm{E}-03 & 25.463 & 1.10 \mathrm{E}+01 & 226.99 \\ 1.200 \mathrm{E}-02 & 30.910 & 1.20 \mathrm{E}+01 & 247.60 \\ 1.500 \mathrm{E}-02 & 23.624 & 1.30 \mathrm{E}+01 & 260.61 \\ 1.900 \mathrm{E}-02 & 13.545 & 1.40 \mathrm{E}+01 & 270.02 \\ 2.550 \mathrm{E}-02 & 9.243 & 1.50 \mathrm{E}+01 & 276.02 \\ 3.200 \mathrm{E}-02 & 5.764 & 1.60 \mathrm{E}+01 & 284.83 \\ 4.000 \mathrm{E}-02 & 6.028 & 1.70 \mathrm{E}+01 & 291.44 \\ 5.250 \mathrm{E}-02 & 14.110 & 1.80 \mathrm{E}+01 & 297.64 \\ 6.600 \mathrm{E}-02 & 19.721 & 1.90 \mathrm{E}+01 & 303.25 \\ & & 2.00 \mathrm{E}+01 & \end{array}$

\begin{tabular}{|c|c|c|c|}
\hline & & & \\
\hline $\mathrm{EDL}=$ & $40.0 \mathrm{EV}$ & $\mathrm{M}=554 . \mathrm{EV}$ & \\
\hline LOWER & CROSS & LOWER & CROSS \\
\hline $\begin{array}{l}\text { ENERGY } \\
\text { (MEV) }\end{array}$ & $\begin{array}{c}\text { SECTION } \\
(\text { KEV-B) }\end{array}$ & $\begin{array}{l}\text { ENERGY } \\
(\mathrm{MEV})\end{array}$ & $\begin{array}{c}\text { SECTION } \\
(\mathrm{KEV}-\mathrm{B})\end{array}$ \\
\hline 1.000E-10 & 13.124 & $8.80 E-02$ & 32.02 \\
\hline $1.000 \mathrm{E}-09$ & 4.151 & $1.10 \mathrm{E}-01$ & 14.24 \\
\hline $1.000 \mathrm{E}-08$ & 2.171 & $1.35 \mathrm{E}-01$ & 36.23 \\
\hline $2.300 \mathrm{E}-08$ & 1.456 & $1.60 \mathrm{E}-01$ & 24.33 \\
\hline $5.000 \mathrm{E}-08$ & 1.094 & $1.90 \mathrm{E}-01$ & 22.63 \\
\hline $7.600 \mathrm{E}-08$ & 0.889 & $2.20 \mathrm{E}-01$ & 26.77 \\
\hline $1.150 \mathrm{E}-07$ & 0.727 & $2.55 \mathrm{E}-01$ & 20.44 \\
\hline $1.700 \mathrm{E}-07$ & 0.596 & $2.90 \mathrm{E}-01$ & 16.55 \\
\hline $2.550 E-07$ & 0.487 & $3.20 E-01$ & 23.69 \\
\hline $3.800 E-07$ & 0.402 & $3.60 \mathrm{E}-01$ & 20.12 \\
\hline $5.500 \mathrm{E}-07$ & 0.330 & $4.00 \mathrm{E}-01$ & 42.95 \\
\hline $8.400 E-07$ & 0.267 & $4.50 \mathrm{E}-01$ & 40.60 \\
\hline $1.275 E-06$ & 0.218 & $5.00 \mathrm{E}-01$ & 36.08 \\
\hline $1.900 \mathrm{E}-06$ & 0.179 & $5.50 \mathrm{E}-01$ & 41.12 \\
\hline $2.800 \mathrm{E}-06$ & 0.146 & $6.00 \mathrm{E}-01$ & 31.32 \\
\hline $4.250 E-06$ & 0.120 & $6.60 \mathrm{E}-01$ & 29.08 \\
\hline $6.300 \mathrm{E}-06$ & 0.099 & $7.20 \mathrm{E}-01$ & 52.60 \\
\hline $9.200 E-06$ & 0.082 & $7.80 \mathrm{E}-01$ & 50.69 \\
\hline $1.350 \mathrm{E}-05$ & 0.066 & $8.40 \mathrm{E}-01$ & 64.26 \\
\hline $2.100 \mathrm{E}-05$ & 0.055 & $9.20 \mathrm{E}-01$ & 50.85 \\
\hline $3.000 E-05$ & 0.045 & $1.00 \mathrm{E}+00$ & 56.33 \\
\hline $4.500 E-05$ & 0.037 & $1.20 \mathrm{E}+00$ & 82.40 \\
\hline $6.900 E-05$ & 0.030 & $1.40 \mathrm{E}+00$ & 99.54 \\
\hline $1.000 \mathrm{E}-04$ & 0.026 & $1.60 \mathrm{E}+00$ & 98.66 \\
\hline $1.350 E-04$ & 0.023 & $1.80 \mathrm{E}+00$ & 93.53 \\
\hline
\end{tabular}




$\begin{array}{lrrr}1.700 \mathrm{E}-04 & 0.020 & 2.00 \mathrm{E}+00 & 117.71 \\ 2.200 \mathrm{E}-04 & 0.018 & 2.30 \mathrm{E}+00 & 128.52 \\ 2.800 \mathrm{E}-04 & 0.016 & 2.60 \mathrm{E}+00 & 143.22 \\ 3.600 \mathrm{E}-04 & 0.015 & 2.90 \mathrm{E}+00 & 142.01 \\ 4.500 \mathrm{E}-04 & 0.014 & 3.30 \mathrm{E}+00 & 151.20 \\ 5.750 \mathrm{E}-04 & 0.099 & 3.70 \mathrm{E}+00 & 161.50 \\ 7.600 \mathrm{E}-04 & 0.187 & 4.10 \mathrm{E}+00 & 170.19 \\ 9.600 \mathrm{E}-04 & 0.268 & 4.50 \mathrm{E}+00 & 182.78 \\ 1.275 \mathrm{E}-03 & 0.327 & 5.00 \mathrm{E}+00 & 187.57 \\ 1.600 \mathrm{E}-03 & 0.659 & 5.50 \mathrm{E}+00 & 192.96 \\ 2.000 \mathrm{E}-03 & 0.651 & 6.00 \mathrm{E}+00 & 198.15 \\ 2.700 \mathrm{E}-03 & 1.305 & 6.70 \mathrm{E}+00 & 204.14 \\ 3.400 \mathrm{E}-03 & 3.336 & 7.40 \mathrm{E}+00 & 211.54 \\ 4.500 \mathrm{E}-03 & 4.301 & 8.20 \mathrm{E}+00 & 217.14 \\ 5.500 \mathrm{E}-03 & 5.329 & 9.00 \mathrm{E}+00 & 223.65 \\ 7.200 \mathrm{E}-03 & 5.550 & 1.00 \mathrm{E}+01 & 234.96 \\ 9.200 \mathrm{E}-03 & 2.472 & 1.10 \mathrm{E}+01 & 247.08 \\ 1.200 \mathrm{E}-02 & 1.771 & 1.20 \mathrm{E}+01 & 264.62 \\ 1.500 \mathrm{E}-02 & 1.611 & 1.30 \mathrm{E}+01 & 273.16 \\ 1.900 \mathrm{E}-02 & 1.943 & 1.40 \mathrm{E}+01 & 278.61 \\ 2.550 \mathrm{E}-02 & 3.376 & 1.50 \mathrm{E}+01 & 290.78 \\ 3.200 \mathrm{E}-02 & 3.050 & 1.60 \mathrm{E}+01 & 293.23 \\ 4.000 \mathrm{E}-02 & 11.534 & 1.70 \mathrm{E}+01 & 295.73 \\ 5.250 \mathrm{E}-02 & 7.697 & 1.80 \mathrm{E}+01 & 296.76 \\ 6.600 \mathrm{E}-02 & 4.458 & 1.90 \mathrm{E}+01 & 299.07 \\ & & 2.00 \mathrm{E}+01 & \end{array}$

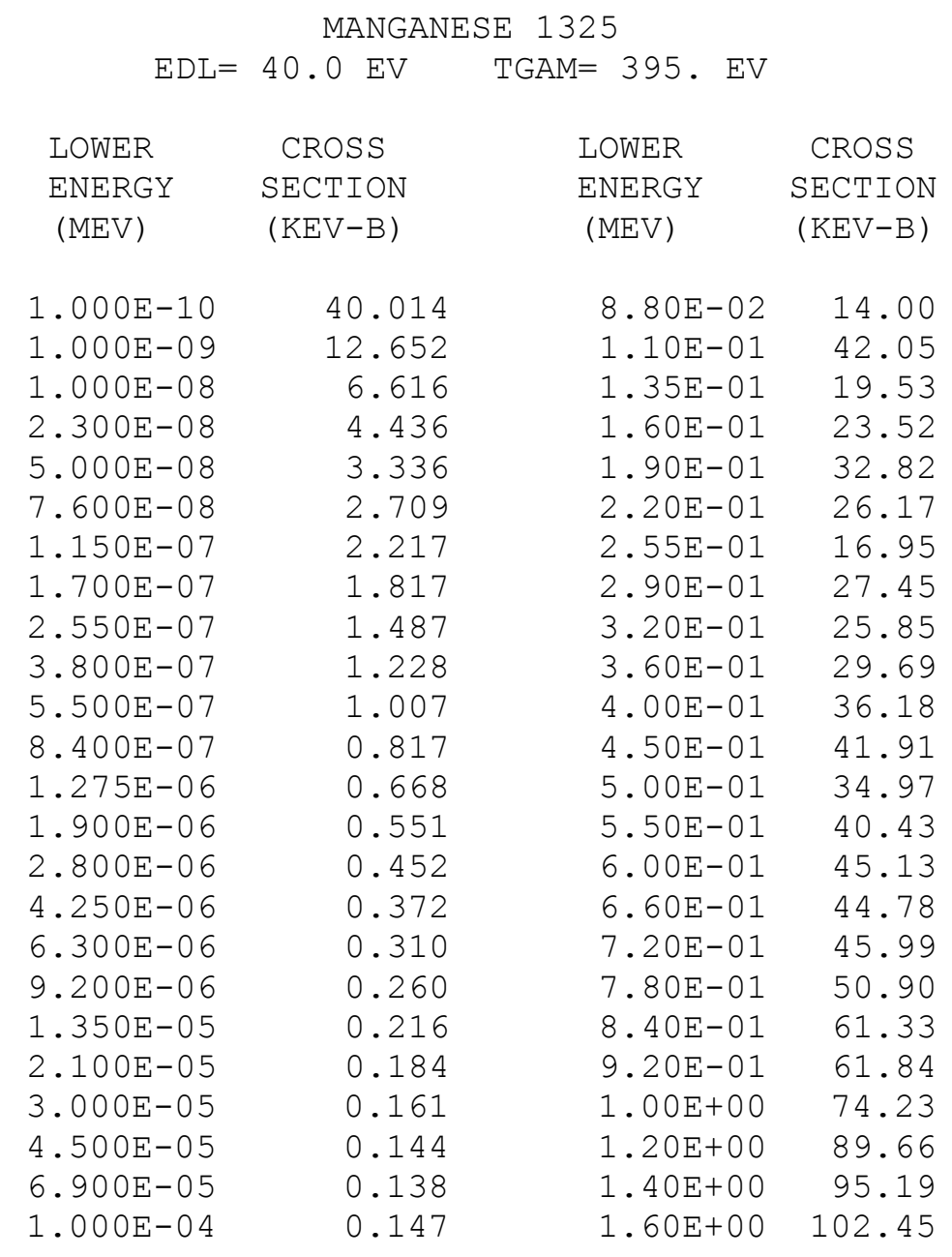




$\begin{array}{lrrr}1.350 \mathrm{E}-04 & 0.173 & 1.80 \mathrm{E}+00 & 108.95 \\ 1.700 \mathrm{E}-04 & 0.249 & 2.00 \mathrm{E}+00 & 114.06 \\ 2.200 \mathrm{E}-04 & 0.597 & 2.30 \mathrm{E}+00 & 119.36 \\ 2.800 \mathrm{E}-04 & 9.403 & 2.60 \mathrm{E}+00 & 127.87 \\ 3.600 \mathrm{E}-04 & 1.137 & 2.90 \mathrm{E}+00 & 138.47 \\ 4.500 \mathrm{E}-04 & 0.122 & 3.30 \mathrm{E}+00 & 147.57 \\ 5.750 \mathrm{E}-04 & 0.253 & 3.70 \mathrm{E}+00 & 159.97 \\ 7.600 \mathrm{E}-04 & 0.600 & 4.10 \mathrm{E}+00 & 154.08 \\ 9.600 \mathrm{E}-04 & 6.673 & 4.50 \mathrm{E}+00 & 159.78 \\ 1.275 \mathrm{E}-03 & 0.909 & 5.00 \mathrm{E}+00 & 169.08 \\ 1.600 \mathrm{E}-03 & 4.077 & 5.50 \mathrm{E}+00 & 175.68 \\ 2.000 \mathrm{E}-03 & 31.236 & 6.00 \mathrm{E}+00 & 182.48 \\ 2.700 \mathrm{E}-03 & 7.958 & 6.70 \mathrm{E}+00 & 191.48 \\ 3.400 \mathrm{E}-03 & 2.104 & 7.40 \mathrm{E}+00 & 199.68 \\ 4.500 \mathrm{E}-03 & 0.990 & 8.20 \mathrm{E}+00 & 199.39 \\ 5.500 \mathrm{E}-03 & 5.103 & 9.00 \mathrm{E}+00 & 204.60 \\ 7.200 \mathrm{E}-03 & 16.702 & 1.00 \mathrm{E}+01 & 218.42 \\ 9.200 \mathrm{E}-03 & 5.293 & 1.10 \mathrm{E}+01 & 237.44 \\ 1.200 \mathrm{E}-02 & 1.654 & 1.20 \mathrm{E}+01 & 249.56 \\ 1.500 \mathrm{E}-02 & 1.617 & 1.30 \mathrm{E}+01 & 257.88 \\ 1.900 \mathrm{E}-02 & 12.079 & 1.40 \mathrm{E}+01 & 259.79 \\ 2.550 \mathrm{E}-02 & 7.289 & 1.50 \mathrm{E}+01 & 270.59 \\ 3.200 \mathrm{E}-02 & 23.335 & 1.60 \mathrm{E}+01 & 279.78 \\ 4.000 \mathrm{E}-02 & 9.897 & 1.70 \mathrm{E}+01 & 284.77 \\ 5.250 \mathrm{E}-02 & 15.472 & 1.80 \mathrm{E}+01 & 293.95 \\ 6.600 \mathrm{E}-02 & 17.663 & 1.90 \mathrm{E}+01 & 304.63 \\ & & 2.00 \mathrm{E}+01 & \end{array}$

\begin{tabular}{|c|c|c|c|}
\hline LOWER & CROSS & LOWER & CROSS \\
\hline $\begin{array}{l}\text { ENERGY } \\
(\mathrm{MEV})\end{array}$ & $\begin{array}{l}\text { SECTION } \\
(\mathrm{KEV}-\mathrm{B})\end{array}$ & $\begin{array}{l}\text { ENERGY } \\
(\mathrm{MEV})\end{array}$ & $\begin{array}{c}\text { SECTION } \\
(\mathrm{KEV}-\mathrm{B})\end{array}$ \\
\hline $1.000 \mathrm{E}-10$ & 7.730 & $8.80 \mathrm{E}-02$ & 11.84 \\
\hline $1.000 E-09$ & 2.444 & $1.10 \mathrm{E}-01$ & 11.27 \\
\hline $1.000 \mathrm{E}-08$ & 1.278 & 1. $35 \mathrm{E}-01$ & 21.53 \\
\hline $2.300 E-08$ & 0.857 & $1.60 \mathrm{E}-01$ & 15.85 \\
\hline $5.000 \mathrm{E}-08$ & 0.644 & $1.90 \mathrm{E}-01$ & 22.01 \\
\hline $7.600 \mathrm{E}-08$ & 0.523 & $2.20 \mathrm{E}-01$ & 18.27 \\
\hline $1.150 \mathrm{E}-07$ & 0.428 & $2.55 \mathrm{E}-01$ & 17.37 \\
\hline $1.700 \mathrm{E}-07$ & 0.351 & $2.90 \mathrm{E}-01$ & 13.51 \\
\hline $2.550 \mathrm{E}-07$ & 0.287 & $3.20 \mathrm{E}-01$ & 25.14 \\
\hline $3.800 \mathrm{E}-07$ & 0.237 & $3.60 \mathrm{E}-01$ & 46.52 \\
\hline $5.500 \mathrm{E}-07$ & 0.194 & $4.00 \mathrm{E}-01$ & 43.04 \\
\hline $8.400 \mathrm{E}-07$ & 0.157 & $4.50 \mathrm{E}-01$ & 36.40 \\
\hline $1.275 \mathrm{E}-06$ & 0.128 & $5.00 \mathrm{E}-01$ & 33.22 \\
\hline $1.900 \mathrm{E}-06$ & 0.105 & $5.50 \mathrm{E}-01$ & 34.18 \\
\hline $2.800 \mathrm{E}-06$ & 0.086 & $6.00 \mathrm{E}-01$ & 19.64 \\
\hline $4.250 \mathrm{E}-06$ & 0.070 & $6.60 \mathrm{E}-01$ & 48.89 \\
\hline $6.300 \mathrm{E}-06$ & 0.058 & $7.20 \mathrm{E}-01$ & 74.22 \\
\hline $9.200 \mathrm{E}-06$ & 0.048 & $7.80 \mathrm{E}-01$ & 44.02 \\
\hline $1.350 \mathrm{E}-05$ & 0.038 & $8.40 \mathrm{E}-01$ & 40.97 \\
\hline $2.100 \mathrm{E}-05$ & 0.031 & $9.20 \mathrm{E}-01$ & 50.95 \\
\hline $3.000 \mathrm{E}-05$ & 0.026 & $1.00 \mathrm{E}+00$ & 50.09 \\
\hline $4.500 \mathrm{E}-05$ & 0.021 & $1.20 \mathrm{E}+00$ & 64.54 \\
\hline $6.900 \mathrm{E}-05$ & 0.017 & $1.40 \mathrm{E}+00$ & 73.58 \\
\hline
\end{tabular}




$\begin{array}{lrrr}1.000 \mathrm{E}-04 & 0.014 & 1.60 \mathrm{E}+00 & 76.46 \\ 1.350 \mathrm{E}-04 & 0.014 & 1.80 \mathrm{E}+00 & 95.15 \\ 1.700 \mathrm{E}-04 & 0.011 & 2.00 \mathrm{E}+00 & 93.75 \\ 2.200 \mathrm{E}-04 & 0.011 & 2.30 \mathrm{E}+00 & 112.05 \\ 2.800 \mathrm{E}-04 & 0.010 & 2.60 \mathrm{E}+00 & 123.55 \\ 3.600 \mathrm{E}-04 & 0.007 & 2.90 \mathrm{E}+00 & 133.45 \\ 4.500 \mathrm{E}-04 & 0.005 & 3.30 \mathrm{E}+00 & 135.25 \\ 5.750 \mathrm{E}-04 & 0.129 & 3.70 \mathrm{E}+00 & 149.55 \\ 7.600 \mathrm{E}-04 & 0.308 & 4.10 \mathrm{E}+00 & 158.25 \\ 9.600 \mathrm{E}-04 & 0.672 & 4.50 \mathrm{E}+00 & 168.55 \\ 1.275 \mathrm{E}-03 & 0.477 & 5.00 \mathrm{E}+00 & 176.46 \\ 1.600 \mathrm{E}-03 & 0.511 & 5.50 \mathrm{E}+00 & 183.06 \\ 2.000 \mathrm{E}-03 & 0.568 & 6.00 \mathrm{E}+00 & 189.26 \\ 2.700 \mathrm{E}-03 & 0.632 & 6.70 \mathrm{E}+00 & 196.67 \\ 3.400 \mathrm{E}-03 & 0.822 & 7.40 \mathrm{E}+00 & 203.37 \\ 4.500 \mathrm{E}-03 & 0.826 & 8.20 \mathrm{E}+00 & 214.58 \\ 5.500 \mathrm{E}-03 & 1.671 & 9.00 \mathrm{E}+00 & 225.69 \\ 7.200 \mathrm{E}-03 & 3.709 & 1.00 \mathrm{E}+01 & 237.60 \\ 9.200 \mathrm{E}-03 & 1.491 & 1.10 \mathrm{E}+01 & 247.41 \\ 1.200 \mathrm{E}-02 & 1.156 & 1.20 \mathrm{E}+01 & 258.52 \\ 1.500 \mathrm{E}-02 & 0.973 & 1.30 \mathrm{E}+01 & 271.35 \\ 1.900 \mathrm{E}-02 & 0.555 & 1.40 \mathrm{E}+01 & 290.27 \\ 2.550 \mathrm{E}-02 & 26.762 & 1.50 \mathrm{E}+01 & 293.20 \\ 3.200 \mathrm{E}-02 & 8.161 & 1.60 \mathrm{E}+01 & 292.73 \\ 4.000 \mathrm{E}-02 & 6.515 & 1.70 \mathrm{E}+01 & 297.65 \\ 5.250 \mathrm{E}-02 & 6.576 & 1.80 \mathrm{E}+01 & 307.26 \\ 6.600 \mathrm{E}-02 & 13.137 & 1.90 \mathrm{E}+01 & 316.36 \\ & & 2.00 \mathrm{E}+01 & \end{array}$

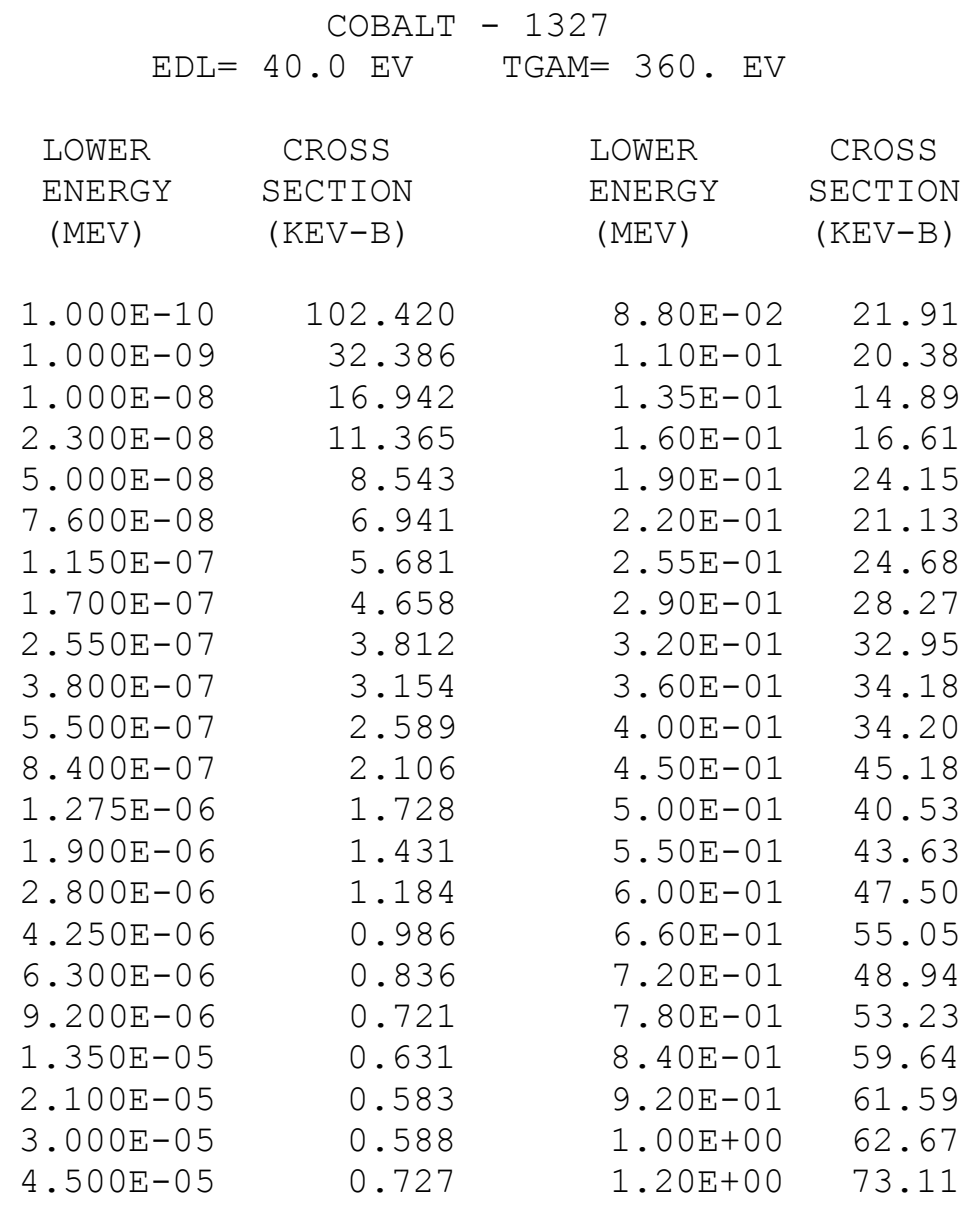




$\begin{array}{lrrr}6.900 \mathrm{E}-05 & 1.524 & 1.40 \mathrm{E}+00 & 77.00 \\ 1.000 \mathrm{E}-04 & 58.432 & 1.60 \mathrm{E}+00 & 82.11 \\ 1.350 \mathrm{E}-04 & 16.307 & 1.80 \mathrm{E}+00 & 86.65 \\ 1.700 \mathrm{E}-04 & 0.631 & 2.00 \mathrm{E}+00 & 92.59 \\ 2.200 \mathrm{E}-04 & 0.153 & 2.30 \mathrm{E}+00 & 103.13 \\ 2.800 \mathrm{E}-04 & 0.058 & 2.60 \mathrm{E}+00 & 100.24 \\ 3.600 \mathrm{E}-04 & 0.028 & 2.90 \mathrm{E}+00 & 108.04 \\ 4.500 \mathrm{E}-04 & 0.015 & 3.30 \mathrm{E}+00 & 118.35 \\ 5.750 \mathrm{E}-04 & 0.033 & 3.70 \mathrm{E}+00 & 124.36 \\ 7.600 \mathrm{E}-04 & 0.077 & 4.10 \mathrm{E}+00 & 133.47 \\ 9.600 \mathrm{E}-04 & 0.094 & 4.50 \mathrm{E}+00 & 148.67 \\ 1.275 \mathrm{E}-03 & 0.098 & 5.00 \mathrm{E}+00 & 162.48 \\ 1.600 \mathrm{E}-03 & 0.084 & 5.50 \mathrm{E}+00 & 170.69 \\ 2.000 \mathrm{E}-03 & 0.095 & 6.00 \mathrm{E}+00 & 178.00 \\ 2.700 \mathrm{E}-03 & 0.244 & 6.70 \mathrm{E}+00 & 186.09 \\ 3.400 \mathrm{E}-03 & 7.530 & 7.40 \mathrm{E}+00 & 194.81 \\ 4.500 \mathrm{E}-03 & 23.447 & 8.20 \mathrm{E}+00 & 202.88 \\ 5.500 \mathrm{E}-03 & 6.123 & 9.00 \mathrm{E}+00 & 208.49 \\ 7.200 \mathrm{E}-03 & 3.330 & 1.00 \mathrm{E}+01 & 215.21 \\ 9.200 \mathrm{E}-03 & 3.425 & 1.10 \mathrm{E}+01 & 227.22 \\ 1.200 \mathrm{E}-02 & 1.916 & 1.20 \mathrm{E}+01 & 244.24 \\ 1.500 \mathrm{E}-02 & 4.683 & 1.30 \mathrm{E}+01 & 268.95 \\ 1.900 \mathrm{E}-02 & 12.376 & 1.40 \mathrm{E}+01 & 294.26 \\ 2.550 \mathrm{E}-02 & 11.431 & 1.50 \mathrm{E}+01 & 313.97 \\ 3.200 \mathrm{E}-02 & 11.296 & 1.60 \mathrm{E}+01 & 333.36 \\ 4.000 \mathrm{E}-02 & 11.192 & 1.70 \mathrm{E}+01 & 345.34 \\ 5.250 \mathrm{E}-02 & 16.081 & 1.80 \mathrm{E}+01 & 351.92 \\ 6.600 \mathrm{E}-02 & 16.002 & 1.90 \mathrm{E}+01 & 361.71 \\ & & 2.00 \mathrm{E}+01 & \end{array}$

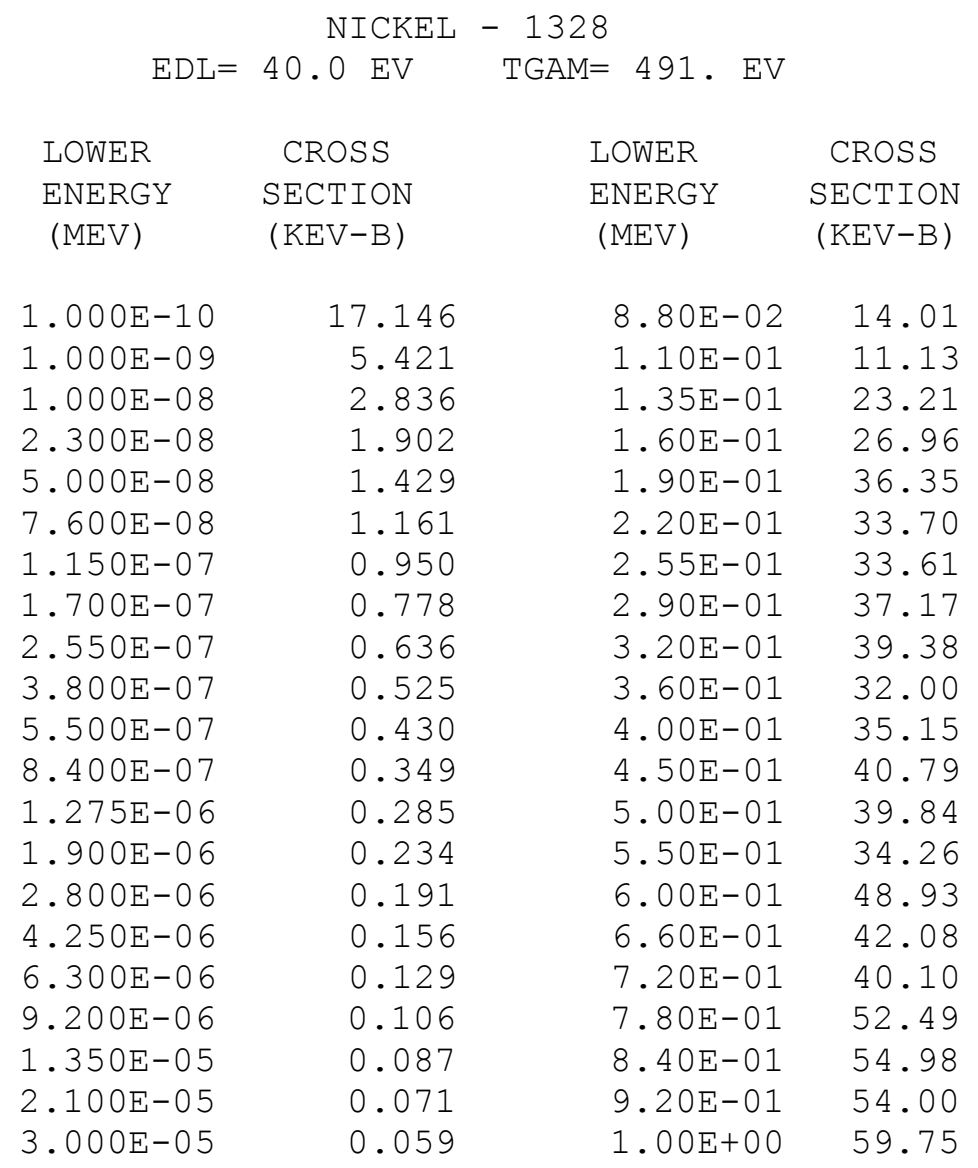




$\begin{array}{lrrr}4.500 \mathrm{E}-05 & 0.048 & 1.20 \mathrm{E}+00 & 80.38 \\ 6.900 \mathrm{E}-05 & 0.039 & 1.40 \mathrm{E}+00 & 80.69 \\ 1.000 \mathrm{E}-04 & 0.033 & 1.60 \mathrm{E}+00 & 77.53 \\ 1.350 \mathrm{E}-04 & 0.029 & 1.80 \mathrm{E}+00 & 82.03 \\ 1.700 \mathrm{E}-04 & 0.026 & 2.00 \mathrm{E}+00 & 91.54 \\ 2.200 \mathrm{E}-04 & 0.023 & 2.30 \mathrm{E}+00 & 102.03 \\ 2.800 \mathrm{E}-04 & 0.021 & 2.60 \mathrm{E}+00 & 113.83 \\ 3.600 \mathrm{E}-04 & 0.018 & 2.90 \mathrm{E}+00 & 113.04 \\ 4.500 \mathrm{E}-04 & 0.017 & 3.30 \mathrm{E}+00 & 127.34 \\ 5.750 \mathrm{E}-04 & 0.159 & 3.70 \mathrm{E}+00 & 132.14 \\ 7.600 \mathrm{E}-04 & 0.489 & 4.10 \mathrm{E}+00 & 160.45 \\ 9.600 \mathrm{E}-04 & 0.758 & 4.50 \mathrm{E}+00 & 168.66 \\ 1.275 \mathrm{E}-03 & 0.881 & 5.00 \mathrm{E}+00 & 179.88 \\ 1.600 \mathrm{E}-03 & 0.979 & 5.50 \mathrm{E}+00 & 186.99 \\ 2.000 \mathrm{E}-03 & 1.208 & 6.00 \mathrm{E}+00 & 207.51 \\ 2.700 \mathrm{E}-03 & 1.588 & 6.70 \mathrm{E}+00 & 218.93 \\ 3.400 \mathrm{E}-03 & 2.993 & 7.40 \mathrm{E}+00 & 223.75 \\ 4.500 \mathrm{E}-03 & 4.089 & 8.20 \mathrm{E}+00 & 231.66 \\ 5.500 \mathrm{E}-03 & 2.606 & 9.00 \mathrm{E}+00 & 256.66 \\ 7.200 \mathrm{E}-03 & 2.022 & 1.00 \mathrm{E}+01 & 269.45 \\ 9.200 \mathrm{E}-03 & 5.491 & 1.10 \mathrm{E}+01 & 281.94 \\ 1.200 \mathrm{E}-02 & 17.723 & 1.20 \mathrm{E}+01 & 289.52 \\ 1.500 \mathrm{E}-02 & 30.591 & 1.30 \mathrm{E}+01 & 296.51 \\ 1.900 \mathrm{E}-02 & 10.225 & 1.40 \mathrm{E}+01 & 300.19 \\ 2.550 \mathrm{E}-02 & 10.413 & 1.50 \mathrm{E}+01 & 303.49 \\ 3.200 \mathrm{E}-02 & 8.737 & 1.60 \mathrm{E}+01 & 309.09 \\ 4.000 \mathrm{E}-02 & 7.021 & 1.70 \mathrm{E}+01 & 324.69 \\ 5.250 \mathrm{E}-02 & 14.677 & 1.80 \mathrm{E}+01 & 333.60 \\ 6.600 \mathrm{E}-02 & 16.898 & 1.90 \mathrm{E}+01 & 337.61 \\ & & 2.00 \mathrm{E}+01 & \end{array}$

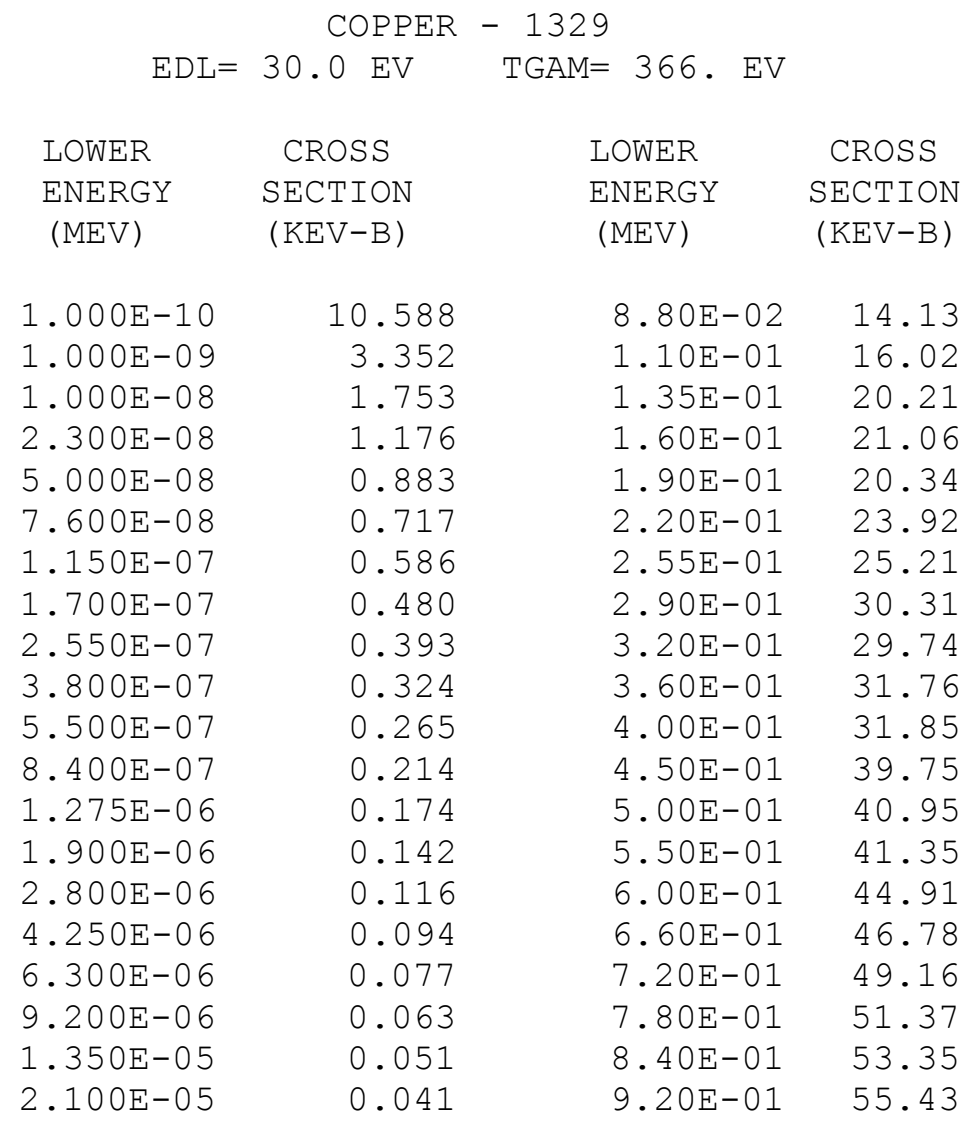




$\begin{array}{lrrr}3.000 \mathrm{E}-05 & 0.033 & 1.00 \mathrm{E}+00 & 58.77 \\ 4.500 \mathrm{E}-05 & 0.026 & 1.20 \mathrm{E}+00 & 65.96 \\ 6.900 \mathrm{E}-05 & 0.021 & 1.40 \mathrm{E}+00 & 66.37 \\ 1.000 \mathrm{E}-04 & 0.018 & 1.60 \mathrm{E}+00 & 72.07 \\ 1.350 \mathrm{E}-04 & 0.016 & 1.80 \mathrm{E}+00 & 79.20 \\ 1.700 \mathrm{E}-04 & 0.016 & 2.00 \mathrm{E}+00 & 86.43 \\ 2.200 \mathrm{E}-04 & 0.340 & 2.30 \mathrm{E}+00 & 95.25 \\ 2.800 \mathrm{E}-04 & 0.014 & 2.60 \mathrm{E}+00 & 103.48 \\ 3.600 \mathrm{E}-04 & 0.017 & 2.90 \mathrm{E}+00 & 112.88 \\ 4.500 \mathrm{E}-04 & 0.266 & 3.30 \mathrm{E}+00 & 122.79 \\ 5.750 \mathrm{E}-04 & 2.002 & 3.70 \mathrm{E}+00 & 132.50 \\ 7.600 \mathrm{E}-04 & 0.152 & 4.10 \mathrm{E}+00 & 146.71 \\ 9.600 \mathrm{E}-04 & 0.225 & 4.50 \mathrm{E}+00 & 157.72 \\ 1.275 \mathrm{E}-03 & 0.250 & 5.00 \mathrm{E}+00 & 164.63 \\ 1.600 \mathrm{E}-03 & 0.401 & 5.50 \mathrm{E}+00 & 172.04 \\ 2.000 \mathrm{E}-03 & 3.623 & 6.00 \mathrm{E}+00 & 180.85 \\ 2.700 \mathrm{E}-03 & 0.634 & 6.70 \mathrm{E}+00 & 186.77 \\ 3.400 \mathrm{E}-03 & 0.989 & 7.40 \mathrm{E}+00 & 198.38 \\ 4.500 \mathrm{E}-03 & 2.852 & 8.20 \mathrm{E}+00 & 209.10 \\ 5.500 \mathrm{E}-03 & 1.601 & 9.00 \mathrm{E}+00 & 223.02 \\ 7.200 \mathrm{E}-03 & 4.355 & 1.00 \mathrm{E}+01 & 245.64 \\ 9.200 \mathrm{E}-03 & 4.080 & 1.10 \mathrm{E}+01 & 260.36 \\ 1.200 \mathrm{E}-02 & 4.723 & 1.20 \mathrm{E}+01 & 272.08 \\ 1.500 \mathrm{E}-02 & 6.225 & 1.30 \mathrm{E}+01 & 284.80 \\ 1.900 \mathrm{E}-02 & 7.596 & 1.40 \mathrm{E}+01 & 296.41 \\ 2.550 \mathrm{E}-02 & 9.963 & 1.50 \mathrm{E}+01 & 306.43 \\ 3.200 \mathrm{E}-02 & 8.164 & 1.60 \mathrm{E}+01 & 313.75 \\ 4.000 \mathrm{E}-02 & 7.594 & 1.70 \mathrm{E}+01 & 321.76 \\ 5.250 \mathrm{E}-02 & 11.882 & 1.80 \mathrm{E}+01 & 332.88 \\ 6.600 \mathrm{E}-02 & 14.333 & 1.90 \mathrm{E}+01 & 347.39 \\ & & 2.00 \mathrm{E}+01 & \end{array}$

\begin{tabular}{|c|c|c|c|}
\hline \multicolumn{4}{|c|}{ ZIRCONIUM 1340} \\
\hline $\mathrm{EDL}=$ & $40.0 \mathrm{EV}$ & $\mathrm{M}=142 . \mathrm{EV}$ & \\
\hline LOWER & CROSS & LOWER & CROSS \\
\hline $\begin{array}{l}\text { ENERGY } \\
\text { (MEV) }\end{array}$ & $\begin{array}{c}\text { SECTION } \\
(\mathrm{KEV}-\mathrm{B})\end{array}$ & $\begin{array}{l}\text { ENERGY } \\
(\mathrm{MEV})\end{array}$ & $\begin{array}{c}\text { SECTION } \\
(\mathrm{KEV}-\mathrm{B})\end{array}$ \\
\hline $1.000 \mathrm{E}-10$ & 0.197 & $8.80 E-02$ & 16.31 \\
\hline $1.000 E-09$ & 0.062 & $1.10 \mathrm{E}-01$ & 18.66 \\
\hline $1.000 E-08$ & 0.033 & $1.35 E-01$ & 22.77 \\
\hline $2.300 E-08$ & 0.022 & $1.60 \mathrm{E}-01$ & 26.39 \\
\hline $5.000 E-08$ & 0.016 & $1.90 \mathrm{E}-01$ & 26.40 \\
\hline $7.600 \mathrm{E}-08$ & 0.013 & $2.20 \mathrm{E}-01$ & 32.35 \\
\hline $1.150 \mathrm{E}-07$ & 0.011 & $2.55 \mathrm{E}-01$ & 32.55 \\
\hline $1.700 \mathrm{E}-07$ & 0.009 & $2.90 \mathrm{E}-01$ & 33.55 \\
\hline $2.550 \mathrm{E}-07$ & 0.007 & $3.20 \mathrm{E}-01$ & 38.52 \\
\hline $3.800 E-07$ & 0.006 & $3.60 E-01$ & 43.68 \\
\hline $5.500 \mathrm{E}-07$ & 0.005 & $4.00 \mathrm{E}-01$ & 50.61 \\
\hline $8.400 \mathrm{E}-07$ & 0.004 & $4.50 \mathrm{E}-01$ & 50.34 \\
\hline $1.275 E-06$ & 0.003 & $5.00 \mathrm{E}-01$ & 53.74 \\
\hline $1.900 \mathrm{E}-06$ & 0.003 & $5.50 \mathrm{E}-01$ & 54.54 \\
\hline $2.800 E-06$ & 0.002 & $6.00 \mathrm{E}-01$ & 57.87 \\
\hline $4.250 \mathrm{E}-06$ & 0.002 & $6.60 \mathrm{E}-01$ & 63.34 \\
\hline $6.300 \mathrm{E}-06$ & 0.001 & $7.20 \mathrm{E}-01$ & 62.03 \\
\hline $9.200 \mathrm{E}-06$ & 0.001 & $7.80 \mathrm{E}-01$ & 61.87 \\
\hline 1. $350 \mathrm{E}-05$ & 0.001 & $8.40 \mathrm{E}-01$ & 68.38 \\
\hline
\end{tabular}




$\begin{array}{lrrr}2.100 \mathrm{E}-05 & 0.001 & 9.20 \mathrm{E}-01 & 67.80 \\ 3.000 \mathrm{E}-05 & 0.001 & 1.00 \mathrm{E}+00 & 71.83 \\ 4.500 \mathrm{E}-05 & 0.000 & 1.20 \mathrm{E}+00 & 76.83 \\ 6.900 \mathrm{E}-05 & 0.000 & 1.40 \mathrm{E}+00 & 81.41 \\ 1.000 \mathrm{E}-04 & 0.000 & 1.60 \mathrm{E}+00 & 87.55 \\ 1.350 \mathrm{E}-04 & 0.000 & 1.80 \mathrm{E}+00 & 91.56 \\ 1.700 \mathrm{E}-04 & 0.035 & 2.00 \mathrm{E}+00 & 96.00 \\ 2.200 \mathrm{E}-04 & 0.009 & 2.30 \mathrm{E}+00 & 101.98 \\ 2.800 \mathrm{E}-04 & 0.222 & 2.60 \mathrm{E}+00 & 107.44 \\ 3.600 \mathrm{E}-04 & 0.004 & 2.90 \mathrm{E}+00 & 113.82 \\ 4.500 \mathrm{E}-04 & 0.000 & 3.30 \mathrm{E}+00 & 119.62 \\ 5.750 \mathrm{E}-04 & 0.041 & 3.70 \mathrm{E}+00 & 125.02 \\ 7.600 \mathrm{E}-04 & 0.008 & 4.10 \mathrm{E}+00 & 131.52 \\ 9.600 \mathrm{E}-04 & 0.077 & 4.50 \mathrm{E}+00 & 139.99 \\ 1.275 \mathrm{E}-03 & 0.306 & 5.00 \mathrm{E}+00 & 148.77 \\ 1.600 \mathrm{E}-03 & 0.252 & 5.50 \mathrm{E}+00 & 157.66 \\ 2.000 \mathrm{E}-03 & 0.331 & 6.00 \mathrm{E}+00 & 167.26 \\ 2.700 \mathrm{E}-03 & 0.979 & 6.70 \mathrm{E}+00 & 177.15 \\ 3.400 \mathrm{E}-03 & 1.051 & 7.40 \mathrm{E}+00 & 187.04 \\ 4.500 \mathrm{E}-03 & 0.810 & 8.20 \mathrm{E}+00 & 198.64 \\ 5.500 \mathrm{E}-03 & 1.577 & 9.00 \mathrm{E}+00 & 210.94 \\ 7.200 \mathrm{E}-03 & 1.486 & 1.00 \mathrm{E}+01 & 224.15 \\ 9.200 \mathrm{E}-03 & 1.124 & 1.10 \mathrm{E}+01 & 235.46 \\ 1.200 \mathrm{E}-02 & 2.636 & 1.20 \mathrm{E}+01 & 247.58 \\ 1.500 \mathrm{E}-02 & 4.030 & 1.30 \mathrm{E}+01 & 254.20 \\ 1.900 \mathrm{E}-02 & 3.729 & 1.40 \mathrm{E}+01 & 259.01 \\ 2.550 \mathrm{E}-02 & 3.670 & 1.50 \mathrm{E}+01 & 262.91 \\ 3.200 \mathrm{E}-02 & 4.781 & 1.60 \mathrm{E}+01 & 270.72 \\ 4.000 \mathrm{E}-02 & 11.364 & 1.70 \mathrm{E}+01 & 288.62 \\ 5.250 \mathrm{E}-02 & 11.111 & 1.80 \mathrm{E}+01 & 312.01 \\ 6.600 \mathrm{E}-02 & 15.782 & 1.90 \mathrm{E}+01 & 343.61 \\ & & 2.00 \mathrm{E}+01 & \\ & & & \end{array}$

NIOBIUM 1189

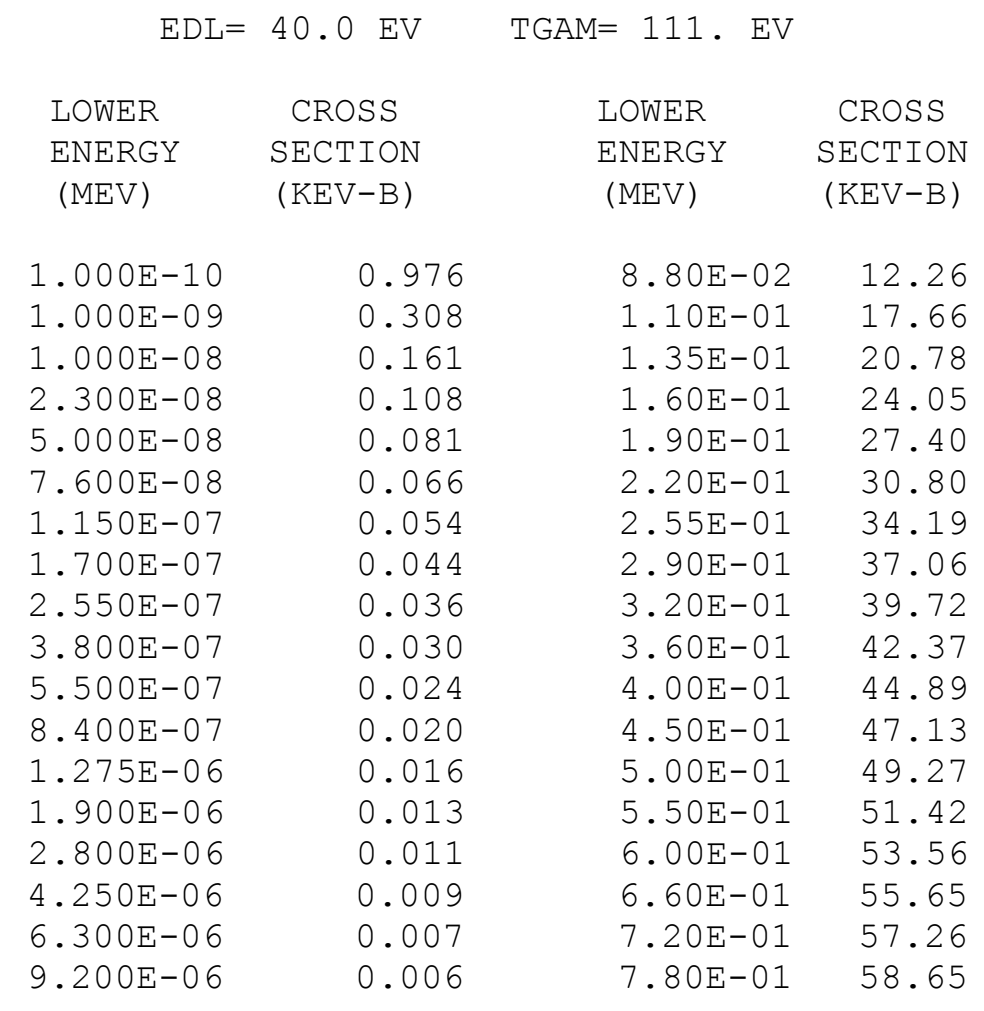




$\begin{array}{lllr}1.350 \mathrm{E}-05 & 0.005 & 8.40 \mathrm{E}-01 & 59.12 \\ 2.100 \mathrm{E}-05 & 0.004 & 9.20 \mathrm{E}-01 & 59.75 \\ 3.000 \mathrm{E}-05 & 0.082 & 1.00 \mathrm{E}+00 & 60.85 \\ 4.500 \mathrm{E}-05 & 0.003 & 1.20 \mathrm{E}+00 & 64.28 \\ 6.900 \mathrm{E}-05 & 0.030 & 1.40 \mathrm{E}+00 & 68.15 \\ 1.000 \mathrm{E}-04 & 0.248 & 1.60 \mathrm{E}+00 & 75.30 \\ 1.350 \mathrm{E}-04 & 0.003 & 1.80 \mathrm{E}+00 & 82.37 \\ 1.700 \mathrm{E}-04 & 0.708 & 2.00 \mathrm{E}+00 & 89.13 \\ 2.200 \mathrm{E}-04 & 0.037 & 2.30 \mathrm{E}+00 & 96.56 \\ 2.800 \mathrm{E}-04 & 0.147 & 2.60 \mathrm{E}+00 & 101.43 \\ 3.600 \mathrm{E}-04 & 0.500 & 2.90 \mathrm{E}+00 & 107.13 \\ 4.500 \mathrm{E}-04 & 0.051 & 3.30 \mathrm{E}+00 & 112.42 \\ 5.750 \mathrm{E}-04 & 0.234 & 3.70 \mathrm{E}+00 & 120.51 \\ 7.600 \mathrm{E}-04 & 0.219 & 4.10 \mathrm{E}+00 & 130.11 \\ 9.600 \mathrm{E}-04 & 0.467 & 4.50 \mathrm{E}+00 & 140.50 \\ 1.275 \mathrm{E}-03 & 0.589 & 5.00 \mathrm{E}+00 & 149.60 \\ 1.600 \mathrm{E}-03 & 0.502 & 5.50 \mathrm{E}+00 & 158.00 \\ 2.000 \mathrm{E}-03 & 0.952 & 6.00 \mathrm{E}+00 & 170.31 \\ 2.700 \mathrm{E}-03 & 0.607 & 6.70 \mathrm{E}+00 & 181.91 \\ 3.400 \mathrm{E}-03 & 0.720 & 7.40 \mathrm{E}+00 & 191.51 \\ 4.500 \mathrm{E}-03 & 0.921 & 8.20 \mathrm{E}+00 & 200.11 \\ 5.500 \mathrm{E}-03 & 1.281 & 9.00 \mathrm{E}+00 & 207.81 \\ 7.200 \mathrm{E}-03 & 1.113 & 1.00 \mathrm{E}+01 & 218.80 \\ 9.200 \mathrm{E}-03 & 1.380 & 1.10 \mathrm{E}+01 & 228.70 \\ 1.200 \mathrm{E}-02 & 1.700 & 1.20 \mathrm{E}+01 & 237.90 \\ 1.500 \mathrm{E}-02 & 2.092 & 1.30 \mathrm{E}+01 & 251.00 \\ 1.900 \mathrm{E}-02 & 2.664 & 1.40 \mathrm{E}+01 & 271.00 \\ 2.550 \mathrm{E}-02 & 3.364 & 1.50 \mathrm{E}+01 & 295.60 \\ 3.200 \mathrm{E}-02 & 4.135 & 1.60 \mathrm{E}+01 & 313.41 \\ 4.000 \mathrm{E}-02 & 5.191 & 1.70 \mathrm{E}+01 & 332.31 \\ 5.250 \mathrm{E}-02 & 6.508 & 1.80 \mathrm{E}+01 & 350.21 \\ 6.600 \mathrm{E}-02 & 8.239 & 1.90 \mathrm{E}+01 & 367.52 \\ & & 2.00 \mathrm{E}+01 & \end{array}$

MOLY 1321

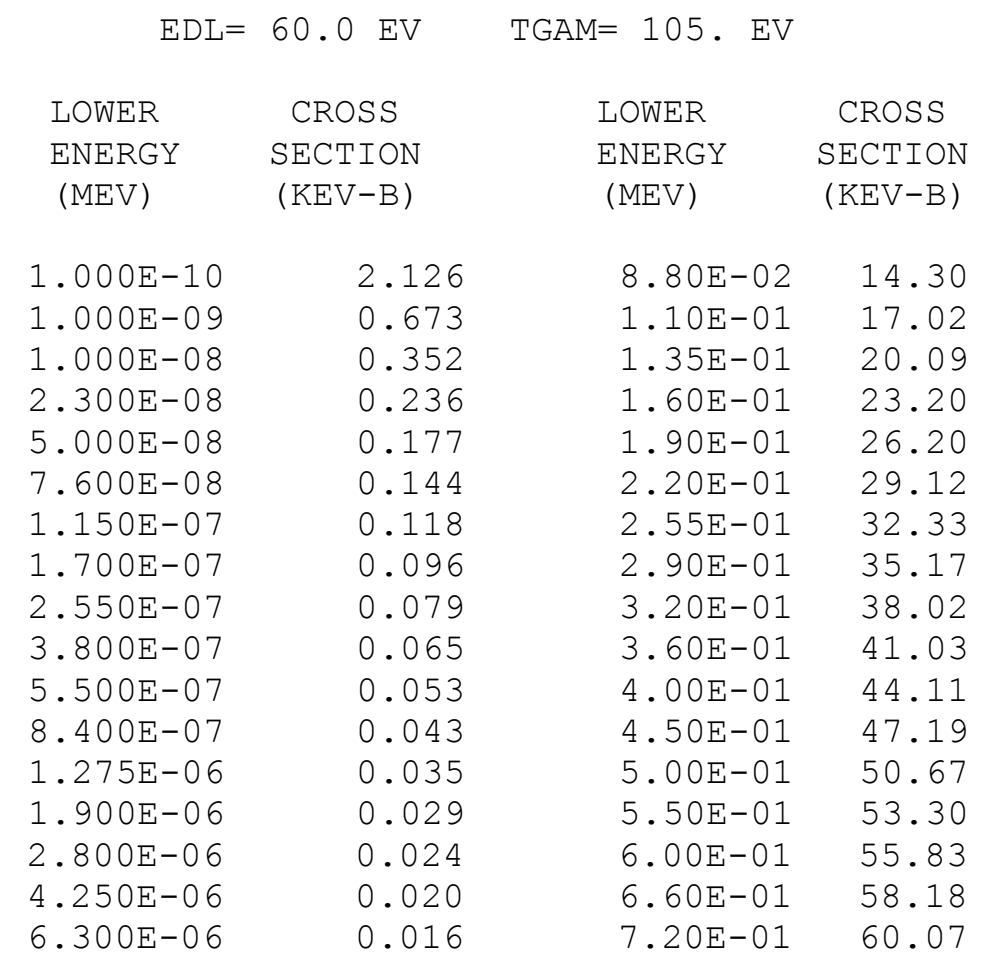




$\begin{array}{lrrr}9.200 \mathrm{E}-06 & 0.254 & 7.80 \mathrm{E}-01 & 62.03 \\ 1.350 \mathrm{E}-05 & 0.015 & 8.40 \mathrm{E}-01 & 63.61 \\ 2.100 \mathrm{E}-05 & 0.019 & 9.20 \mathrm{E}-01 & 65.03 \\ 3.000 \mathrm{E}-05 & 2.688 & 1.00 \mathrm{E}+00 & 70.91 \\ 4.500 \mathrm{E}-05 & 1.669 & 1.20 \mathrm{E}+00 & 76.95 \\ 6.900 \mathrm{E}-05 & 0.154 & 1.40 \mathrm{E}+00 & 83.67 \\ 1.000 \mathrm{E}-04 & 1.751 & 1.60 \mathrm{E}+00 & 89.09 \\ 1.350 \mathrm{E}-04 & 0.106 & 1.80 \mathrm{E}+00 & 92.19 \\ 1.700 \mathrm{E}-04 & 0.007 & 2.00 \mathrm{E}+00 & 95.15 \\ 2.200 \mathrm{E}-04 & 0.034 & 2.30 \mathrm{E}+00 & 98.08 \\ 2.800 \mathrm{E}-04 & 0.238 & 2.60 \mathrm{E}+00 & 102.73 \\ 3.600 \mathrm{E}-04 & 0.385 & 2.90 \mathrm{E}+00 & 108.23 \\ 4.500 \mathrm{E}-04 & 0.371 & 3.30 \mathrm{E}+00 & 113.36 \\ 5.750 \mathrm{E}-04 & 0.122 & 3.70 \mathrm{E}+00 & 118.40 \\ 7.600 \mathrm{E}-04 & 0.144 & 4.10 \mathrm{E}+00 & 124.41 \\ 9.600 \mathrm{E}-04 & 0.841 & 4.50 \mathrm{E}+00 & 132.41 \\ 1.275 \mathrm{E}-03 & 0.852 & 5.00 \mathrm{E}+00 & 141.95 \\ 1.600 \mathrm{E}-03 & 2.841 & 5.50 \mathrm{E}+00 & 150.90 \\ 2.000 \mathrm{E}-03 & 4.642 & 6.00 \mathrm{E}+00 & 160.49 \\ 2.700 \mathrm{E}-03 & 5.647 & 6.70 \mathrm{E}+00 & 169.34 \\ 3.400 \mathrm{E}-03 & 6.034 & 7.40 \mathrm{E}+00 & 183.43 \\ 4.500 \mathrm{E}-03 & 6.654 & 8.20 \mathrm{E}+00 & 199.02 \\ 5.500 \mathrm{E}-03 & 7.540 & 9.00 \mathrm{E}+00 & 213.40 \\ 7.200 \mathrm{E}-03 & 8.867 & 1.00 \mathrm{E}+01 & 224.48 \\ 9.200 \mathrm{E}-03 & 10.512 & 1.10 \mathrm{E}+01 & 239.77 \\ 1.200 \mathrm{E}-02 & 12.434 & 1.20 \mathrm{E}+01 & 253.55 \\ 1.500 \mathrm{E}-02 & 14.602 & 1.30 \mathrm{E}+01 & 257.73 \\ 1.900 \mathrm{E}-02 & 17.537 & 1.40 \mathrm{E}+01 & 259.35 \\ 2.550 \mathrm{E}-02 & 20.542 & 1.50 \mathrm{E}+01 & 268.93 \\ 3.200 \mathrm{E}-02 & 23.163 & 1.60 \mathrm{E}+01 & 276.12 \\ 4.000 \mathrm{E}-02 & 25.473 & 1.70 \mathrm{E}+01 & 290.50 \\ 5.250 \mathrm{E}-02 & 25.053 & 1.80 \mathrm{E}+01 & 308.80 \\ 6.600 \mathrm{E}-02 & 21.531 & 1.90 \mathrm{E}+01 & 321.39 \\ & & 2.00 \mathrm{E}+01 & \end{array}$

\begin{tabular}{|c|c|c|c|}
\hline & SI & 371 & \\
\hline $\mathrm{EDL}=$ & $60.0 \mathrm{EV}$ & $\mathrm{M}=143 . \mathrm{EV}$ & \\
\hline LOWER & CROSS & LOWER & CROSS \\
\hline ENERGY & SECTION & ENERGY & SECTION \\
\hline$(\mathrm{MEV})$ & $(\mathrm{KEV}-\mathrm{B})$ & $(\mathrm{MEV})$ & $(\mathrm{KEV}-\mathrm{B})$ \\
\hline $1.000 \mathrm{E}-10$ & 40.269 & $8.80 E-02$ & 11.65 \\
\hline $1.000 \mathrm{E}-09$ & 12.733 & $1.10 \mathrm{E}-01$ & 13.95 \\
\hline $1.000 \mathrm{E}-08$ & 6.660 & $1.35 \mathrm{E}-01$ & 16.17 \\
\hline $2.300 \mathrm{E}-08$ & 4.467 & $1.60 \mathrm{E}-01$ & 18.43 \\
\hline $5.000 \mathrm{E}-08$ & 3.358 & $1.90 \mathrm{E}-01$ & 20.66 \\
\hline $7.600 \mathrm{E}-08$ & 2.727 & $2.20 \mathrm{E}-01$ & 23.01 \\
\hline $1.150 E-07$ & 2.232 & $2.55 \mathrm{E}-01$ & 25.25 \\
\hline $1.700 \mathrm{E}-07$ & 1.829 & $2.90 \mathrm{E}-01$ & 27.08 \\
\hline $2.550 \mathrm{E}-07$ & 1.497 & $3.20 \mathrm{E}-01$ & 28.62 \\
\hline $3.800 \mathrm{E}-07$ & 1.238 & $3.60 \mathrm{E}-01$ & 30.36 \\
\hline $5.500 \mathrm{E}-07$ & 1.015 & $4.00 \mathrm{E}-01$ & 31.86 \\
\hline $8.400 E-07$ & 0.825 & $4.50 \mathrm{E}-01$ & 33.51 \\
\hline $1.275 E-06$ & 0.676 & $5.00 \mathrm{E}-01$ & 35.22 \\
\hline $1.900 \mathrm{E}-06$ & 0.559 & $5.50 \mathrm{E}-01$ & 37.23 \\
\hline $2.800 \mathrm{E}-06$ & 0.463 & $6.00 \mathrm{E}-01$ & 39.12 \\
\hline $4.250 \mathrm{E}-06$ & 0.388 & $6.60 \mathrm{E}-01$ & 41.25 \\
\hline
\end{tabular}




$\begin{array}{lrrr}6.300 \mathrm{E}-06 & 0.342 & 7.20 \mathrm{E}-01 & 43.13 \\ 9.200 \mathrm{E}-06 & 0.388 & 7.80 \mathrm{E}-01 & 45.20 \\ 1.350 \mathrm{E}-05 & 13.106 & 8.40 \mathrm{E}-01 & 47.50 \\ 2.100 \mathrm{E}-05 & 0.206 & 9.20 \mathrm{E}-01 & 50.08 \\ 3.000 \mathrm{E}-05 & 4.916 & 1.00 \mathrm{E}+00 & 55.04 \\ 4.500 \mathrm{E}-05 & 7.622 & 1.20 \mathrm{E}+00 & 61.36 \\ 6.900 \mathrm{E}-05 & 0.095 & 1.40 \mathrm{E}+00 & 67.86 \\ 1.000 \mathrm{E}-04 & 0.090 & 1.60 \mathrm{E}+00 & 73.95 \\ 1.350 \mathrm{E}-04 & 0.609 & 1.80 \mathrm{E}+00 & 79.04 \\ 1.700 \mathrm{E}-04 & 2.132 & 2.00 \mathrm{E}+00 & 84.20 \\ 2.200 \mathrm{E}-04 & 0.308 & 2.30 \mathrm{E}+00 & 90.43 \\ 2.800 \mathrm{E}-04 & 1.189 & 2.60 \mathrm{E}+00 & 96.06 \\ 3.600 \mathrm{E}-04 & 0.551 & 2.90 \mathrm{E}+00 & 101.81 \\ 4.500 \mathrm{E}-04 & 1.013 & 3.30 \mathrm{E}+00 & 107.64 \\ 5.750 \mathrm{E}-04 & 0.762 & 3.70 \mathrm{E}+00 & 115.04 \\ 7.600 \mathrm{E}-04 & 0.221 & 4.10 \mathrm{E}+00 & 122.92 \\ 9.600 \mathrm{E}-04 & 0.591 & 4.50 \mathrm{E}+00 & 131.74 \\ 1.275 \mathrm{E}-03 & 0.587 & 5.00 \mathrm{E}+00 & 141.09 \\ 1.600 \mathrm{E}-03 & 0.680 & 5.50 \mathrm{E}+00 & 149.90 \\ 2.000 \mathrm{E}-03 & 0.947 & 6.00 \mathrm{E}+00 & 159.55 \\ 2.700 \mathrm{E}-03 & 1.121 & 6.70 \mathrm{E}+00 & 169.54 \\ 3.400 \mathrm{E}-03 & 1.163 & 7.40 \mathrm{E}+00 & 178.82 \\ 4.500 \mathrm{E}-03 & 1.233 & 8.20 \mathrm{E}+00 & 186.46 \\ 5.500 \mathrm{E}-03 & 1.353 & 9.00 \mathrm{E}+00 & 189.00 \\ 7.200 \mathrm{E}-03 & 1.549 & 1.00 \mathrm{E}+01 & 197.55 \\ 9.200 \mathrm{E}-03 & 1.804 & 1.10 \mathrm{E}+01 & 207.15 \\ 1.200 \mathrm{E}-02 & 2.130 & 1.20 \mathrm{E}+01 & 213.30 \\ 1.500 \mathrm{E}-02 & 2.552 & 1.30 \mathrm{E}+01 & 222.45 \\ 1.900 \mathrm{E}-02 & 3.175 & 1.40 \mathrm{E}+01 & 236.55 \\ 2.550 \mathrm{E}-02 & 3.961 & 1.50 \mathrm{E}+01 & 249.15 \\ 3.200 \mathrm{E}-02 & 4.804 & 1.60 \mathrm{E}+01 & 256.65 \\ 4.000 \mathrm{E}-02 & 5.985 & 1.70 \mathrm{E}+01 & 263.70 \\ 5.250 \mathrm{E}-02 & 7.464 & 1.80 \mathrm{E}+01 & 272.55 \\ 6.600 \mathrm{E}-02 & 9.386 & 1.90 \mathrm{E}+01 & 281.40 \\ & & 2.00 \mathrm{E}+01 & \end{array}$

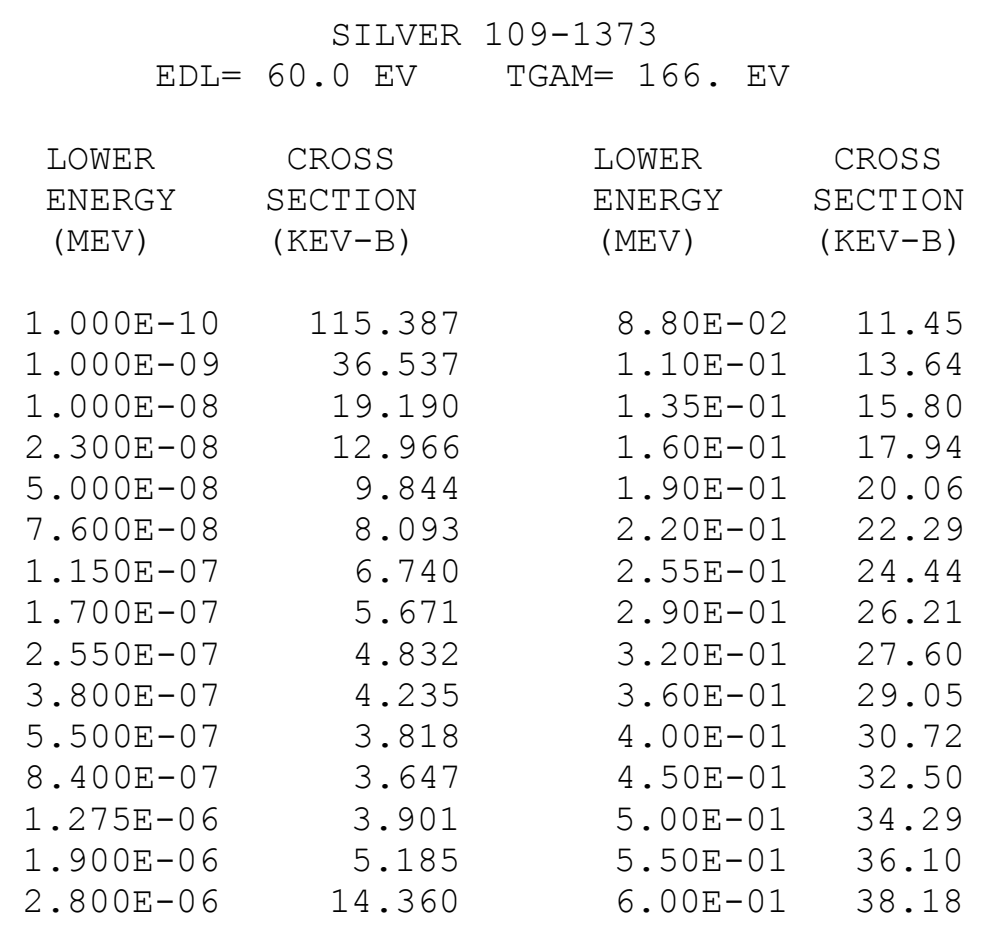




$\begin{array}{rrrr}4.250 \mathrm{E}-06 & 536.305 & 6.60 \mathrm{E}-01 & 40.51 \\ 6.300 \mathrm{E}-06 & 5.264 & 7.20 \mathrm{E}-01 & 42.47 \\ 9.200 \mathrm{E}-06 & 0.608 & 7.80 \mathrm{E}-01 & 44.57 \\ 1.350 \mathrm{E}-05 & 0.164 & 8.40 \mathrm{E}-01 & 46.75 \\ 2.100 \mathrm{E}-05 & 0.750 & 9.20 \mathrm{E}-01 & 49.57 \\ 3.000 \mathrm{E}-05 & 11.531 & 1.00 \mathrm{E}+00 & 54.40 \\ 4.500 \mathrm{E}-05 & 3.491 & 1.20 \mathrm{E}+00 & 60.81 \\ 6.900 \mathrm{E}-05 & 6.393 & 1.40 \mathrm{E}+00 & 66.40 \\ 1.000 \mathrm{E}-04 & 5.203 & 1.60 \mathrm{E}+00 & 71.85 \\ 1.350 \mathrm{E}-04 & 0.356 & 1.80 \mathrm{E}+00 & 76.66 \\ 1.700 \mathrm{E}-04 & 3.024 & 2.00 \mathrm{E}+00 & 81.81 \\ 2.200 \mathrm{E}-04 & 0.496 & 2.30 \mathrm{E}+00 & 87.17 \\ 2.800 \mathrm{E}-04 & 1.933 & 2.60 \mathrm{E}+00 & 91.94 \\ 3.600 \mathrm{E}-04 & 1.318 & 2.90 \mathrm{E}+00 & 97.00 \\ 4.500 \mathrm{E}-04 & 1.922 & 3.30 \mathrm{E}+00 & 103.53 \\ 5.750 \mathrm{E}-04 & 0.827 & 3.70 \mathrm{E}+00 & 111.13 \\ 7.600 \mathrm{E}-04 & 0.386 & 4.10 \mathrm{E}+00 & 119.30 \\ 9.600 \mathrm{E}-04 & 0.793 & 4.50 \mathrm{E}+00 & 128.81 \\ 1.275 \mathrm{E}-03 & 0.778 & 5.00 \mathrm{E}+00 & 138.85 \\ 1.600 \mathrm{E}-03 & 0.814 & 5.50 \mathrm{E}+00 & 147.95 \\ 2.000 \mathrm{E}-03 & 1.090 & 6.00 \mathrm{E}+00 & 157.44 \\ 2.700 \mathrm{E}-03 & 1.245 & 6.70 \mathrm{E}+00 & 166.54 \\ 3.400 \mathrm{E}-03 & 1.300 & 7.40 \mathrm{E}+00 & 174.02 \\ 4.500 \mathrm{E}-03 & 1.350 & 8.20 \mathrm{E}+00 & 181.36 \\ 5.500 \mathrm{E}-03 & 1.454 & 9.00 \mathrm{E}+00 & 182.40 \\ 7.200 \mathrm{E}-03 & 1.638 & 1.00 \mathrm{E}+01 & 194.40 \\ 9.200 \mathrm{E}-03 & 1.906 & 1.10 \mathrm{E}+01 & 198.45 \\ 1.200 \mathrm{E}-02 & 2.235 & 1.20 \mathrm{E}+01 & 206.25 \\ 1.500 \mathrm{E}-02 & 2.656 & 1.30 \mathrm{E}+01 & 212.55 \\ 1.900 \mathrm{E}-02 & 3.279 & 1.40 \mathrm{E}+01 & 222.00 \\ 2.550 \mathrm{E}-02 & 4.041 & 1.50 \mathrm{E}+01 & 230.40 \\ 3.200 \mathrm{E}-02 & 4.877 & 1.60 \mathrm{E}+01 & 240.15 \\ 4.000 \mathrm{E}-02 & 6.034 & 1.70 \mathrm{E}+01 & 251.55 \\ 5.250 \mathrm{E}-02 & 7.463 & 1.80 \mathrm{E}+01 & 261.60 \\ 6.600 \mathrm{E}-02 & 9.314 & 1.90 \mathrm{E}+01 & 270.00 \\ & & 2.00 \mathrm{E}+01 & \end{array}$

TANTALUM 1285

$\mathrm{EDL}=53.0 \mathrm{EV} \quad \mathrm{TGAM}=19 . \mathrm{EV}$

$\begin{array}{lc}\text { LOWER } & \text { CROSS } \\ \text { ENERGY } & \text { SECTION } \\ (\text { MEV }) & (\text { KEV-B })\end{array}$

$\begin{array}{ll}1.000 \mathrm{E}-10 & 3.046 \\ 1.000 \mathrm{E}-09 & 0.964 \\ 1.000 \mathrm{E}-08 & 0.506 \\ 2.300 \mathrm{E}-08 & 0.341 \\ 5.000 \mathrm{E}-08 & 0.258 \\ 7.600 \mathrm{E}-08 & 0.212 \\ 1.150 \mathrm{E}-07 & 0.176 \\ 1.700 \mathrm{E}-07 & 0.147 \\ 2.550 \mathrm{E}-07 & 0.124 \\ 3.800 \mathrm{E}-07 & 0.107 \\ 5.500 \mathrm{E}-07 & 0.096 \\ 8.400 \mathrm{E}-07 & 0.090 \\ 1.275 \mathrm{E}-06 & 0.097 \\ 1.900 \mathrm{E}-06 & 0.145\end{array}$

\section{LOWER CROSS \\ ENERGY SECTION \\ (MEV) (KEV-B)}

$\begin{array}{rr}8.80 \mathrm{E}-02 & 6.93 \\ 1.10 \mathrm{E}-01 & 7.88 \\ 1.35 \mathrm{E}-01 & 8.81 \\ 1.60 \mathrm{E}-01 & 9.87 \\ 1.90 \mathrm{E}-01 & 11.04 \\ 2.20 \mathrm{E}-01 & 12.33 \\ 2.55 \mathrm{E}-01 & 13.59 \\ 2.90 \mathrm{E}-01 & 14.71 \\ 3.20 \mathrm{E}-01 & 15.91 \\ 3.60 \mathrm{E}-01 & 17.16 \\ 4.00 \mathrm{E}-01 & 18.68 \\ 4.50 \mathrm{E}-01 & 20.50 \\ 5.00 \mathrm{E}-01 & 22.16 \\ 5.50 \mathrm{E}-01 & 23.88\end{array}$




\begin{tabular}{|c|c|c|c|}
\hline $2.800 \mathrm{E}-06$ & 6.218 & $6.00 \mathrm{E}-01$ & 25.69 \\
\hline $4.250 \mathrm{E}-06$ & 13.758 & $6.60 \mathrm{E}-01$ & 27.72 \\
\hline $6.300 E-06$ & 0.066 & $7.20 \mathrm{E}-01$ & 29.95 \\
\hline $9.200 E-06$ & 3.315 & $7.80 \mathrm{E}-01$ & 31.09 \\
\hline $1.350 E-05$ & 0.713 & $8.40 E-01$ & 32.75 \\
\hline $2.100 \mathrm{E}-05$ & 1.111 & $9.20 \mathrm{E}-01$ & 35.09 \\
\hline $3.000 E-05$ & 3.306 & $1.00 \mathrm{E}+00$ & 40.11 \\
\hline $4.500 \mathrm{E}-05$ & 0.191 & $1.20 \mathrm{E}+00$ & 46.90 \\
\hline $6.900 \mathrm{E}-05$ & 1.095 & 1. $40 \mathrm{E}+00$ & 53.43 \\
\hline $1.000 \mathrm{E}-04$ & 0.694 & $1.60 \mathrm{E}+00$ & 57.88 \\
\hline $1.350 E-04$ & 0.361 & $1.80 \mathrm{E}+00$ & 60.82 \\
\hline $1.700 \mathrm{E}-04$ & 0.834 & $2.00 \mathrm{E}+00$ & 64.74 \\
\hline $2.200 E-04$ & 0.549 & $2.30 \mathrm{E}+00$ & 69.93 \\
\hline $2.800 E-04$ & 0.410 & $2.60 \mathrm{E}+00$ & 73.43 \\
\hline $3.600 \mathrm{E}-04$ & 0.349 & $2.90 \mathrm{E}+00$ & 76.99 \\
\hline $4.500 \mathrm{E}-04$ & 0.322 & $3.30 \mathrm{E}+00$ & 80.93 \\
\hline $5.750 \mathrm{E}-04$ & 0.293 & $3.70 \mathrm{E}+00$ & 83.84 \\
\hline $7.600 \mathrm{E}-04$ & 0.254 & $4.10 \mathrm{E}+00$ & 89.62 \\
\hline $9.600 \mathrm{E}-04$ & 0.219 & $4.50 \mathrm{E}+00$ & 96.73 \\
\hline $1.275 \mathrm{E}-03$ & 0.190 & $5.00 \mathrm{E}+00$ & 103.44 \\
\hline $1.600 \mathrm{E}-03$ & 0.166 & $5.50 \mathrm{E}+00$ & 108.79 \\
\hline $2.000 \mathrm{E}-03$ & 0.145 & $6.00 \mathrm{E}+00$ & 116.01 \\
\hline $2.700 \mathrm{E}-03$ & 0.131 & $6.70 \mathrm{E}+00$ & 125.27 \\
\hline $3.400 \mathrm{E}-03$ & 0.120 & $7.40 \mathrm{E}+00$ & 134.48 \\
\hline $4.500 \mathrm{E}-03$ & 0.586 & $8.20 \mathrm{E}+00$ & 150.49 \\
\hline $5.500 \mathrm{E}-03$ & 1.040 & $9.00 \mathrm{E}+00$ & 166.57 \\
\hline $7.200 \mathrm{E}-03$ & 1.370 & $1.00 \mathrm{E}+01$ & 178.94 \\
\hline $9.200 \mathrm{E}-03$ & 1.531 & $1.10 \mathrm{E}+01$ & 184.19 \\
\hline $1.200 \mathrm{E}-02$ & 1.712 & $1.20 \mathrm{E}+01$ & 195.73 \\
\hline $1.500 \mathrm{E}-02$ & 1.951 & $1.30 \mathrm{E}+01$ & 205.88 \\
\hline $1.900 \mathrm{E}-02$ & 2.288 & $1.40 \mathrm{E}+01$ & 215.76 \\
\hline $2.550 \mathrm{E}-02$ & 2.735 & $1.50 \mathrm{E}+01$ & 224.99 \\
\hline $3.200 \mathrm{E}-02$ & 3.309 & $1.60 \mathrm{E}+01$ & 232.93 \\
\hline $4.000 \mathrm{E}-02$ & 4.101 & $1.70 \mathrm{E}+01$ & 240.13 \\
\hline $5.250 \mathrm{E}-02$ & 4.937 & $1.80 \mathrm{E}+01$ & 250.48 \\
\hline \multirow[t]{2}{*}{$6.600 \mathrm{E}-02$} & 5.850 & $1.90 \mathrm{E}+01$ & 259.25 \\
\hline & & $2.00 \mathrm{E}+01$ & \\
\hline
\end{tabular}

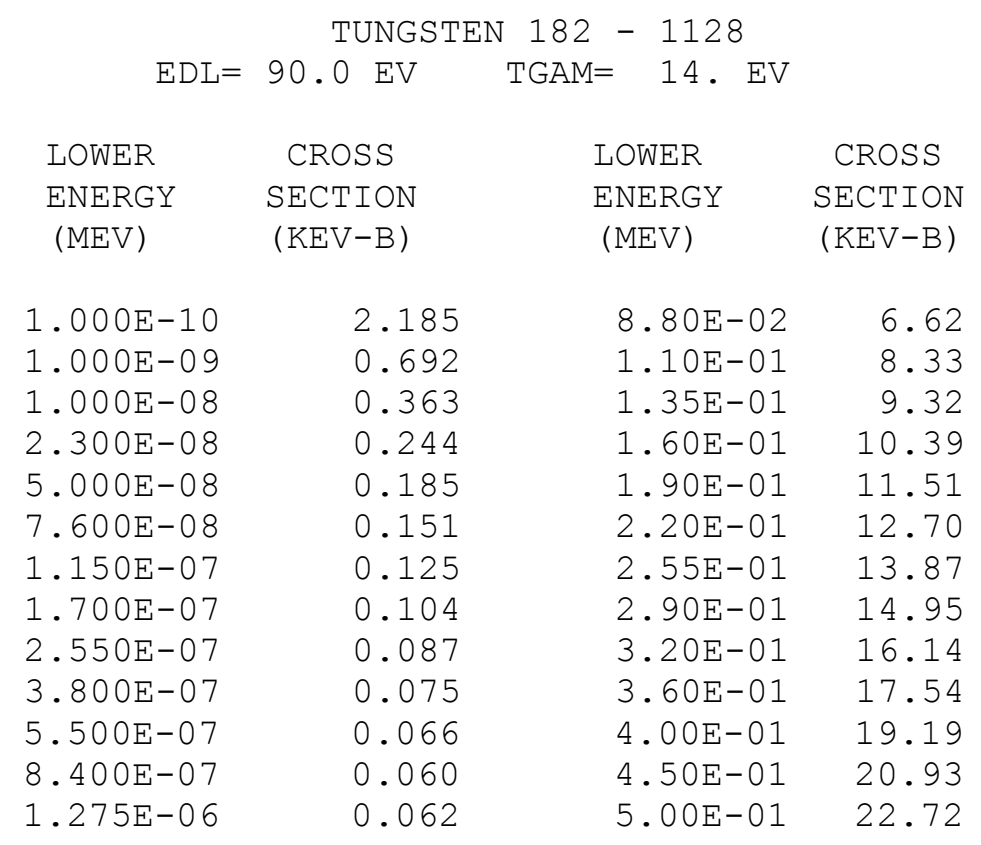




$\begin{array}{lrrr}1.900 \mathrm{E}-06 & 0.088 & 5.50 \mathrm{E}-01 & 24.51 \\ 2.800 \mathrm{E}-06 & 12.479 & 6.00 \mathrm{E}-01 & 26.53 \\ 4.250 \mathrm{E}-06 & 0.811 & 6.60 \mathrm{E}-01 & 28.57 \\ 6.300 \mathrm{E}-06 & 0.026 & 7.20 \mathrm{E}-01 & 30.84 \\ 9.200 \mathrm{E}-06 & 0.022 & 7.80 \mathrm{E}-01 & 33.16 \\ 1.350 \mathrm{E}-05 & 1.277 & 8.40 \mathrm{E}-01 & 35.67 \\ 2.100 \mathrm{E}-05 & 6.150 & 9.20 \mathrm{E}-01 & 38.92 \\ 3.000 \mathrm{E}-05 & 0.006 & 1.00 \mathrm{E}+00 & 43.22 \\ 4.500 \mathrm{E}-05 & 0.002 & 1.20 \mathrm{E}+00 & 46.69 \\ 6.900 \mathrm{E}-05 & 0.003 & 1.40 \mathrm{E}+00 & 47.93 \\ 1.000 \mathrm{E}-04 & 0.743 & 1.60 \mathrm{E}+00 & 50.30 \\ 1.350 \mathrm{E}-04 & 0.002 & 1.80 \mathrm{E}+00 & 53.51 \\ 1.700 \mathrm{E}-04 & 0.002 & 2.00 \mathrm{E}+00 & 57.97 \\ 2.200 \mathrm{E}-04 & 0.240 & 2.30 \mathrm{E}+00 & 62.46 \\ 2.800 \mathrm{E}-04 & 0.016 & 2.60 \mathrm{E}+00 & 66.62 \\ 3.600 \mathrm{E}-04 & 0.161 & 2.90 \mathrm{E}+00 & 71.02 \\ 4.500 \mathrm{E}-04 & 0.056 & 3.30 \mathrm{E}+00 & 76.62 \\ 5.750 \mathrm{E}-04 & 0.075 & 3.70 \mathrm{E}+00 & 81.39 \\ 7.600 \mathrm{E}-04 & 0.036 & 4.10 \mathrm{E}+00 & 86.36 \\ 9.600 \mathrm{E}-04 & 0.061 & 4.50 \mathrm{E}+00 & 92.79 \\ 1.275 \mathrm{E}-03 & 0.046 & 5.00 \mathrm{E}+00 & 99.72 \\ 1.600 \mathrm{E}-03 & 0.023 & 5.50 \mathrm{E}+00 & 106.74 \\ 2.000 \mathrm{E}-03 & 0.041 & 6.00 \mathrm{E}+00 & 114.93 \\ 2.700 \mathrm{E}-03 & 0.027 & 6.70 \mathrm{E}+00 & 124.24 \\ 3.400 \mathrm{E}-03 & 0.030 & 7.40 \mathrm{E}+00 & 132.96 \\ 4.500 \mathrm{E}-03 & 0.739 & 8.20 \mathrm{E}+00 & 141.85 \\ 5.500 \mathrm{E}-03 & 1.377 & 9.00 \mathrm{E}+00 & 157.93 \\ 7.200 \mathrm{E}-03 & 1.786 & 1.00 \mathrm{E}+01 & 168.28 \\ 9.200 \mathrm{E}-03 & 1.930 & 1.10 \mathrm{E}+01 & 176.77 \\ 1.200 \mathrm{E}-02 & 2.114 & 1.20 \mathrm{E}+01 & 182.41 \\ 1.500 \mathrm{E}-02 & 2.354 & 1.30 \mathrm{E}+01 & 190.70 \\ 1.900 \mathrm{E}-02 & 2.749 & 1.40 \mathrm{E}+01 & 200.48 \\ 2.550 \mathrm{E}-02 & 3.182 & 1.50 \mathrm{E}+01 & 209.73 \\ 3.200 \mathrm{E}-02 & 3.634 & 1.60 \mathrm{E}+01 & 222.25 \\ 4.000 \mathrm{E}-02 & 4.204 & 1.70 \mathrm{E}+01 & 235.66 \\ 5.250 \mathrm{E}-02 & 4.786 & 1.80 \mathrm{E}+01 & 247.17 \\ 6.600 \mathrm{E}-02 & 5.399 & 1.90 \mathrm{E}+01 & 256.66 \\ & & 2.00 \mathrm{E}+01 & \end{array}$

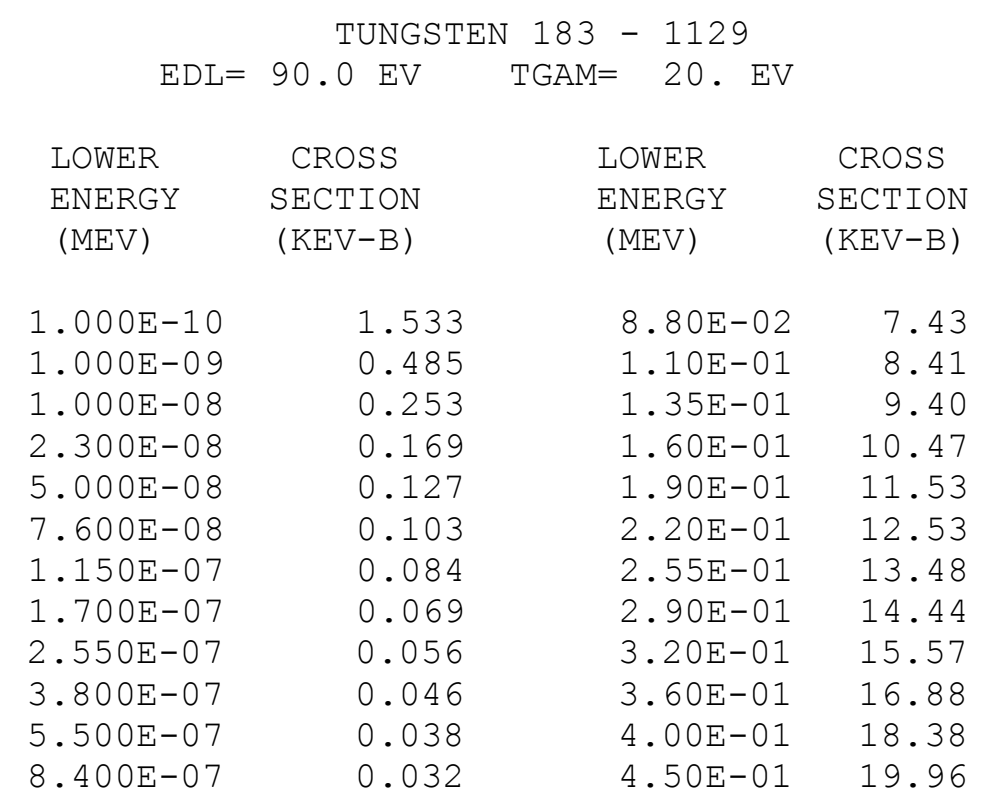




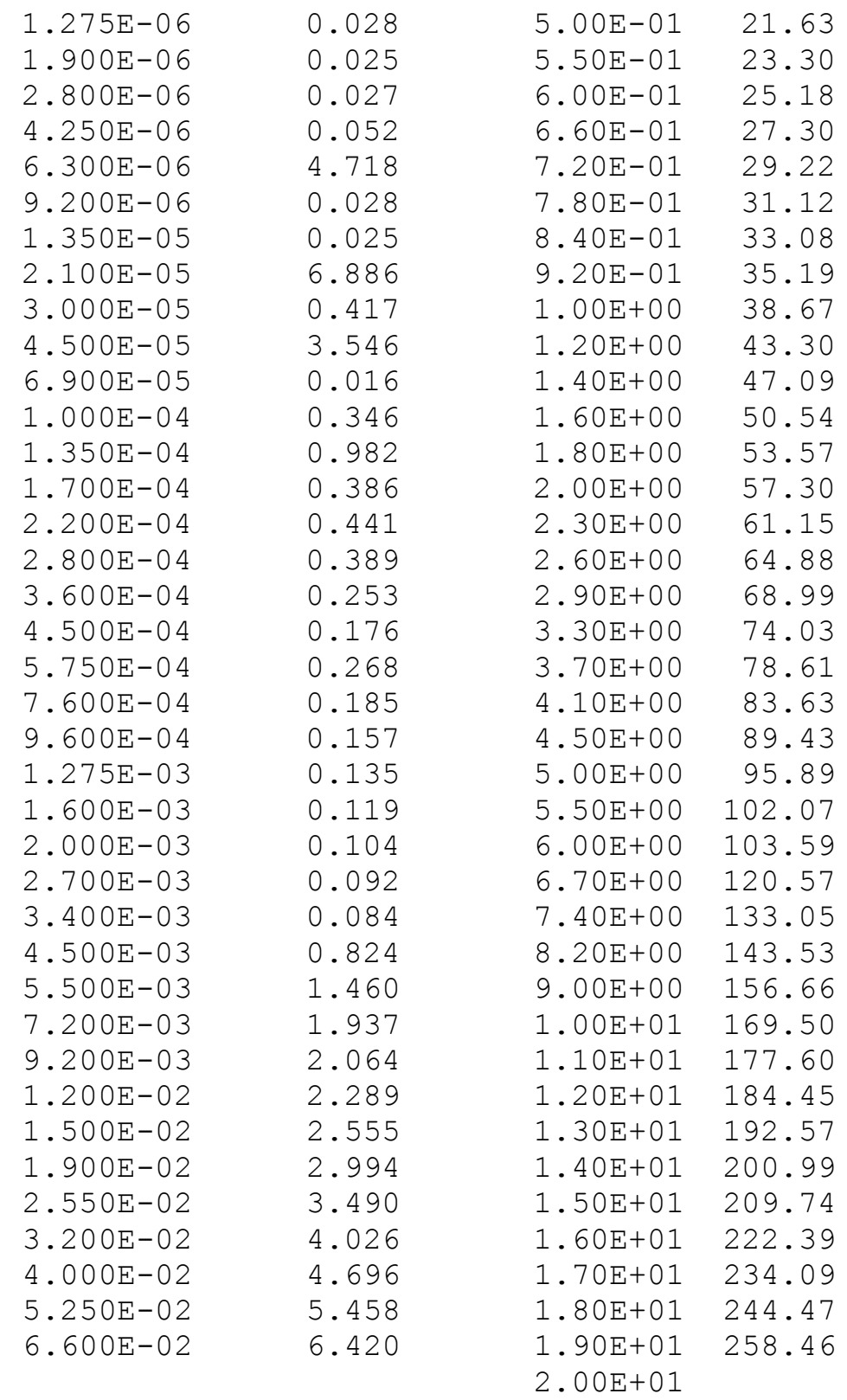

\begin{tabular}{|c|c|c|c|}
\hline $\mathrm{DL}=$ & $\begin{array}{l}\text { TUNGSTE } \\
90.0 \mathrm{EV}\end{array}$ & $\begin{array}{l}\mathrm{N} 184-1130 \\
\mathrm{TGAM}=13 . \mathrm{EV}\end{array}$ & \\
\hline LOWER & CROSS & LOWER & CROSS \\
\hline $\begin{array}{l}\text { ENERGY } \\
(\mathrm{MEV})\end{array}$ & $\begin{array}{l}\text { SECTION } \\
(\text { KEV-B) }\end{array}$ & $\begin{array}{l}\text { ENERGY } \\
(\mathrm{MEV})\end{array}$ & $\begin{array}{l}\text { SECTION } \\
(\mathrm{KEV}-\mathrm{B})\end{array}$ \\
\hline 1.000E-10 & 0.174 & $8.80 \mathrm{E}-02$ & 4.87 \\
\hline $1.000 \mathrm{E}-09$ & 0.055 & $1.10 \mathrm{E}-01$ & 8.45 \\
\hline $1.000 \mathrm{E}-08$ & 0.029 & $1.35 \mathrm{E}-01$ & 9.34 \\
\hline $2.300 \mathrm{E}-08$ & 0.019 & $1.60 \mathrm{E}-01$ & 10.28 \\
\hline $5.000 \mathrm{E}-08$ & 0.014 & $1.90 \mathrm{E}-01$ & 11.29 \\
\hline $7.600 \mathrm{E}-08$ & 0.012 & $2.20 \mathrm{E}-01$ & 12.42 \\
\hline $1.150 \mathrm{E}-07$ & 0.010 & $2.55 \mathrm{E}-01$ & 13.60 \\
\hline $1.700 \mathrm{E}-07$ & 0.008 & $2.90 \mathrm{E}-01$ & 14.68 \\
\hline $2.550 \mathrm{E}-07$ & 0.006 & $3.20 \mathrm{E}-01$ & 15.94 \\
\hline $3.800 \mathrm{E}-07$ & 0.005 & $3.60 \mathrm{E}-01$ & 17.27 \\
\hline $5.500 \mathrm{E}-07$ & 0.004 & $4.00 \mathrm{E}-01$ & 18.89 \\
\hline
\end{tabular}




$\begin{array}{lllr}8.400 \mathrm{E}-07 & 0.003 & 4.50 \mathrm{E}-01 & 20.72 \\ 1.275 \mathrm{E}-06 & 0.003 & 5.00 \mathrm{E}-01 & 22.49 \\ 1.900 \mathrm{E}-06 & 0.002 & 5.50 \mathrm{E}-01 & 24.32 \\ 2.800 \mathrm{E}-06 & 0.002 & 6.00 \mathrm{E}-01 & 26.39 \\ 4.250 \mathrm{E}-06 & 0.001 & 6.60 \mathrm{E}-01 & 28.74 \\ 6.300 \mathrm{E}-06 & 0.001 & 7.20 \mathrm{E}-01 & 31.06 \\ 9.200 \mathrm{E}-06 & 0.001 & 7.80 \mathrm{E}-01 & 33.62 \\ 1.350 \mathrm{E}-05 & 0.001 & 8.40 \mathrm{E}-01 & 36.68 \\ 2.100 \mathrm{E}-05 & 0.001 & 9.20 \mathrm{E}-01 & 39.45 \\ 3.000 \mathrm{E}-05 & 0.000 & 1.00 \mathrm{E}+00 & 43.07 \\ 4.500 \mathrm{E}-05 & 0.001 & 1.20 \mathrm{E}+00 & 46.44 \\ 6.900 \mathrm{E}-05 & 0.001 & 1.40 \mathrm{E}+00 & 48.36 \\ 1.000 \mathrm{E}-04 & 0.049 & 1.60 \mathrm{E}+00 & 52.10 \\ 1.350 \mathrm{E}-04 & 0.005 & 1.80 \mathrm{E}+00 & 55.65 \\ 1.700 \mathrm{E}-04 & 0.363 & 2.00 \mathrm{E}+00 & 59.70 \\ 2.200 \mathrm{E}-04 & 0.001 & 2.30 \mathrm{E}+00 & 63.46 \\ 2.800 \mathrm{E}-04 & 0.074 & 2.60 \mathrm{E}+00 & 66.74 \\ 3.600 \mathrm{E}-04 & 0.047 & 2.90 \mathrm{E}+00 & 70.48 \\ 4.500 \mathrm{E}-04 & 0.000 & 3.30 \mathrm{E}+00 & 75.81 \\ 5.750 \mathrm{E}-04 & 0.040 & 3.70 \mathrm{E}+00 & 81.00 \\ 7.600 \mathrm{E}-04 & 0.060 & 4.10 \mathrm{E}+00 & 86.45 \\ 9.600 \mathrm{E}-04 & 0.066 & 4.50 \mathrm{E}+00 & 92.80 \\ 1.275 \mathrm{E}-03 & 0.043 & 5.00 \mathrm{E}+00 & 99.75 \\ 1.600 \mathrm{E}-03 & 0.032 & 5.50 \mathrm{E}+00 & 106.69 \\ 2.000 \mathrm{E}-03 & 0.035 & 6.00 \mathrm{E}+00 & 114.77 \\ 2.700 \mathrm{E}-03 & 0.025 & 6.70 \mathrm{E}+00 & 123.73 \\ 3.400 \mathrm{E}-03 & 0.023 & 7.40 \mathrm{E}+00 & 123.25 \\ 4.500 \mathrm{E}-03 & 0.794 & 8.20 \mathrm{E}+00 & 144.02 \\ 5.500 \mathrm{E}-03 & 1.517 & 9.00 \mathrm{E}+00 & 157.00 \\ 7.200 \mathrm{E}-03 & 1.994 & 1.00 \mathrm{E}+01 & 166.15 \\ 9.200 \mathrm{E}-03 & 2.080 & 1.10 \mathrm{E}+01 & 173.74 \\ 1.200 \mathrm{E}-02 & 2.252 & 1.20 \mathrm{E}+01 & 182.38 \\ 1.500 \mathrm{E}-02 & 2.450 & 1.30 \mathrm{E}+01 & 190.08 \\ 1.900 \mathrm{E}-02 & 2.745 & 1.40 \mathrm{E}+01 & 196.83 \\ 2.550 \mathrm{E}-02 & 3.036 & 1.50 \mathrm{E}+01 & 205.15 \\ 3.200 \mathrm{E}-02 & 3.278 & 1.60 \mathrm{E}+01 & 216.43 \\ 4.000 \mathrm{E}-02 & 3.461 & 1.70 \mathrm{E}+01 & 228.93 \\ 5.250 \mathrm{E}-02 & 3.482 & 1.80 \mathrm{E}+01 & 243.13 \\ 6.600 \mathrm{E}-02 & 3.205 & 1.90 \mathrm{E}+01 & 253.07 \\ & & 2.00 \mathrm{E}+01 & \end{array}$

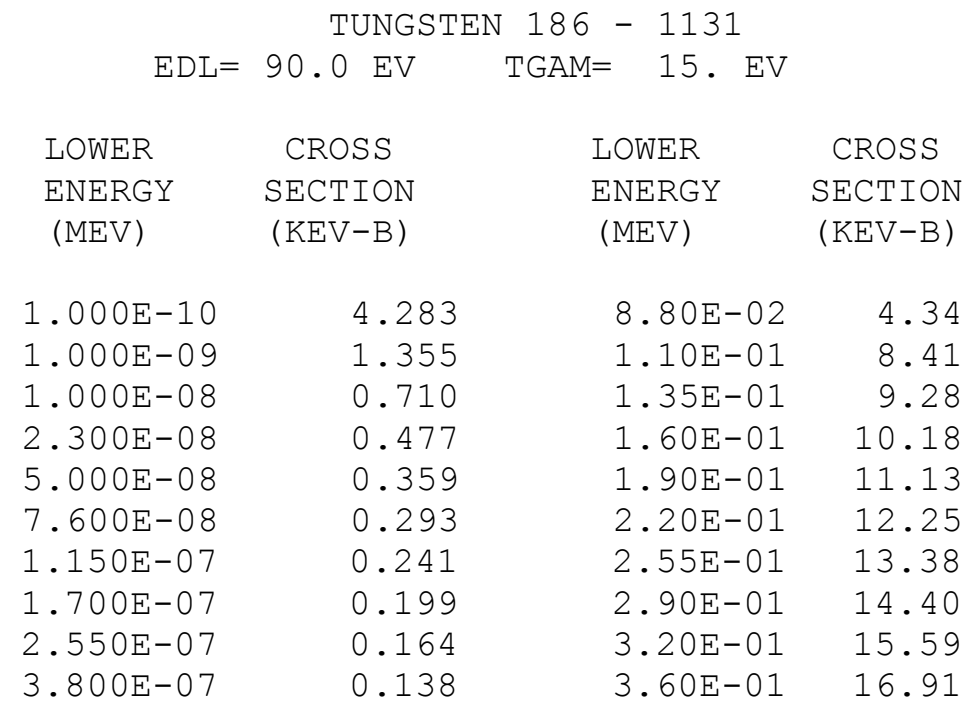




$\begin{array}{lrrr}5.500 \mathrm{E}-07 & 0.116 & 4.00 \mathrm{E}-01 & 18.49 \\ 8.400 \mathrm{E}-07 & 0.098 & 4.50 \mathrm{E}-01 & 20.30 \\ 1.275 \mathrm{E}-06 & 0.085 & 5.00 \mathrm{E}-01 & 22.05 \\ 1.900 \mathrm{E}-06 & 0.076 & 5.50 \mathrm{E}-01 & 23.83 \\ 2.800 \mathrm{E}-06 & 0.072 & 6.00 \mathrm{E}-01 & 25.87 \\ 4.250 \mathrm{E}-06 & 0.075 & 6.60 \mathrm{E}-01 & 28.15 \\ 6.300 \mathrm{E}-06 & 0.094 & 7.20 \mathrm{E}-01 & 30.29 \\ 9.200 \mathrm{E}-06 & 0.180 & 7.80 \mathrm{E}-01 & 32.10 \\ 1.350 \mathrm{E}-05 & 17.833 & 8.40 \mathrm{E}-01 & 34.38 \\ 2.100 \mathrm{E}-05 & 0.267 & 9.20 \mathrm{E}-01 & 36.33 \\ 3.000 \mathrm{E}-05 & 0.018 & 1.00 \mathrm{E}+00 & 39.50 \\ 4.500 \mathrm{E}-05 & 0.004 & 1.20 \mathrm{E}+00 & 44.47 \\ 6.900 \mathrm{E}-05 & 0.001 & 1.40 \mathrm{E}+00 & 48.97 \\ 1.000 \mathrm{E}-04 & 0.001 & 1.60 \mathrm{E}+00 & 53.19 \\ 1.350 \mathrm{E}-04 & 0.006 & 1.80 \mathrm{E}+00 & 56.63 \\ 1.700 \mathrm{E}-04 & 0.477 & 2.00 \mathrm{E}+00 & 60.34 \\ 2.200 \mathrm{E}-04 & 0.025 & 2.30 \mathrm{E}+00 & 63.75 \\ 2.800 \mathrm{E}-04 & 0.059 & 2.60 \mathrm{E}+00 & 66.70 \\ 3.600 \mathrm{E}-04 & 0.063 & 2.90 \mathrm{E}+00 & 70.16 \\ 4.500 \mathrm{E}-04 & 0.092 & 3.30 \mathrm{E}+00 & 75.12 \\ 5.750 \mathrm{E}-04 & 0.104 & 3.70 \mathrm{E}+00 & 79.78 \\ 7.600 \mathrm{E}-04 & 0.014 & 4.10 \mathrm{E}+00 & 85.03 \\ 9.600 \mathrm{E}-04 & 0.055 & 4.50 \mathrm{E}+00 & 91.17 \\ 1.275 \mathrm{E}-03 & 0.026 & 5.00 \mathrm{E}+00 & 98.02 \\ 1.600 \mathrm{E}-03 & 0.014 & 5.50 \mathrm{E}+00 & 104.86 \\ 2.000 \mathrm{E}-03 & 0.026 & 6.00 \mathrm{E}+00 & 112.88 \\ 2.700 \mathrm{E}-03 & 0.023 & 6.70 \mathrm{E}+00 & 121.26 \\ 3.400 \mathrm{E}-03 & 0.016 & 7.40 \mathrm{E}+00 & 127.14 \\ 4.500 \mathrm{E}-03 & 0.726 & 8.20 \mathrm{E}+00 & 139.05 \\ 5.500 \mathrm{E}-03 & 1.434 & 9.00 \mathrm{E}+00 & 154.16 \\ 7.200 \mathrm{E}-03 & 1.901 & 1.00 \mathrm{E}+01 & 163.88 \\ 9.200 \mathrm{E}-03 & 2.022 & 1.10 \mathrm{E}+01 & 170.97 \\ 1.200 \mathrm{E}-02 & 2.144 & 1.20 \mathrm{E}+01 & 178.69 \\ 1.500 \mathrm{E}-02 & 2.346 & 1.30 \mathrm{E}+01 & 186.73 \\ 1.900 \mathrm{E}-02 & 2.605 & 1.40 \mathrm{E}+01 & 194.22 \\ 2.550 \mathrm{E}-02 & 2.850 & 1.50 \mathrm{E}+01 & 204.73 \\ 3.200 \mathrm{E}-02 & 3.031 & 1.60 \mathrm{E}+01 & 217.09 \\ 4.000 \mathrm{E}-02 & 3.119 & 1.70 \mathrm{E}+01 & 228.03 \\ 5.250 \mathrm{E}-02 & 3.006 & 1.80 \mathrm{E}+01 & 238.41 \\ 6.600 \mathrm{E}-02 & 2.520 & 1.90 \mathrm{E}+01 & 253.53 \\ & & 2.00 \mathrm{E}+01 & \\ 1.03 & & \end{array}$

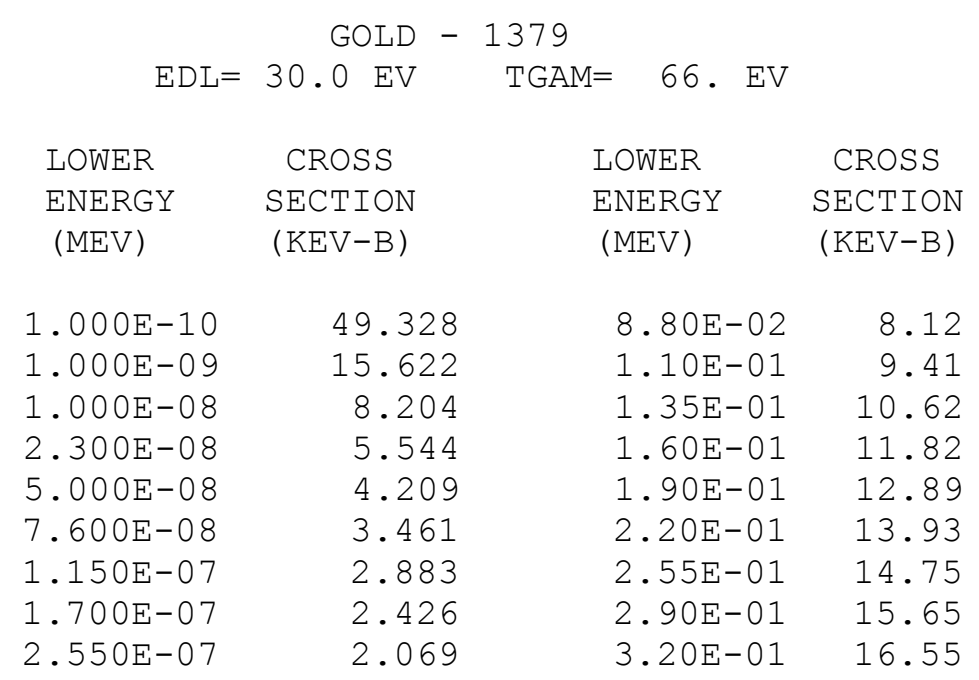




\begin{tabular}{|c|c|c|c|}
\hline $3.800 \mathrm{E}-07$ & 1.815 & $3.60 \mathrm{E}-01$ & 17.56 \\
\hline $5.500 \mathrm{E}-07$ & 1.641 & $4.00 \mathrm{E}-01$ & 18.79 \\
\hline $8.400 \mathrm{E}-07$ & 1.578 & $4.50 \mathrm{E}-01$ & 19.90 \\
\hline $1.275 \mathrm{E}-06$ & 1.715 & $5.00 \mathrm{E}-01$ & 20.74 \\
\hline $1.900 \mathrm{E}-06$ & 2.380 & $5.50 \mathrm{E}-01$ & 21.48 \\
\hline $2.800 E-06$ & 8.476 & $6.00 \mathrm{E}-01$ & 22.13 \\
\hline $4.250 E-06$ & 217.739 & $6.60 \mathrm{E}-01$ & 23.31 \\
\hline $6.300 E-06$ & 1.441 & $7.20 \mathrm{E}-01$ & 24.72 \\
\hline $9.200 \mathrm{E}-06$ & 0.205 & $7.80 \mathrm{E}-01$ & 26.17 \\
\hline $1.350 \mathrm{E}-05$ & 0.054 & $8.40 \mathrm{E}-01$ & 27.82 \\
\hline $2.100 \mathrm{E}-05$ & 0.023 & $9.20 \mathrm{E}-01$ & 29.13 \\
\hline $3.000 \mathrm{E}-05$ & 0.019 & $1.00 \mathrm{E}+00$ & 32.37 \\
\hline $4.500 \mathrm{E}-05$ & 5.534 & $1.20 \mathrm{E}+00$ & 37.98 \\
\hline $6.900 \mathrm{E}-05$ & 0.634 & $1.40 \mathrm{E}+00$ & 43.83 \\
\hline $1.000 \mathrm{E}-04$ & 0.371 & $1.60 \mathrm{E}+00$ & 49.03 \\
\hline $1.350 \mathrm{E}-04$ & 1.672 & $1.80 \mathrm{E}+00$ & 53.72 \\
\hline $1.700 \mathrm{E}-04$ & 0.369 & $2.00 \mathrm{E}+00$ & 59.20 \\
\hline $2.200 E-04$ & 0.968 & $2.30 \mathrm{E}+00$ & 64.84 \\
\hline $2.800 \mathrm{E}-04$ & 1.329 & $2.60 \mathrm{E}+00$ & 71.27 \\
\hline $3.600 \mathrm{E}-04$ & 0.798 & $2.90 \mathrm{E}+00$ & 77.27 \\
\hline $4.500 \mathrm{E}-04$ & 0.651 & $3.30 \mathrm{E}+00$ & 83.10 \\
\hline $5.750 \mathrm{E}-04$ & 1.151 & $3.70 \mathrm{E}+00$ & 86.98 \\
\hline $7.600 \mathrm{E}-04$ & 0.664 & $4.10 \mathrm{E}+00$ & 92.61 \\
\hline $9.600 \mathrm{E}-04$ & 0.554 & $4.50 \mathrm{E}+00$ & 101.39 \\
\hline $1.275 \mathrm{E}-03$ & 0.438 & $5.00 \mathrm{E}+00$ & 107.90 \\
\hline $1.600 \mathrm{E}-03$ & 0.632 & $5.50 \mathrm{E}+00$ & 111.36 \\
\hline $2.000 \mathrm{E}-03$ & 0.914 & $6.00 \mathrm{E}+00$ & 117.67 \\
\hline $2.700 \mathrm{E}-03$ & 0.901 & $6.70 \mathrm{E}+00$ & 125.17 \\
\hline $3.400 \mathrm{E}-03$ & 1.090 & $7.40 \mathrm{E}+00$ & 126.70 \\
\hline $4.500 \mathrm{E}-03$ & 0.960 & $8.20 \mathrm{E}+00$ & 143.64 \\
\hline $5.500 \mathrm{E}-03$ & 1.045 & $9.00 \mathrm{E}+00$ & 168.32 \\
\hline $7.200 \mathrm{E}-03$ & 1.237 & $1.00 \mathrm{E}+01$ & 185.36 \\
\hline $9.200 \mathrm{E}-03$ & 1.490 & $1.10 \mathrm{E}+01$ & 195.44 \\
\hline $1.200 \mathrm{E}-02$ & 1.789 & $1.20 \mathrm{E}+01$ & 202.28 \\
\hline $1.500 \mathrm{E}-02$ & 2.146 & $1.30 \mathrm{E}+01$ & 209.39 \\
\hline $1.900 \mathrm{E}-02$ & 2.642 & $1.40 \mathrm{E}+01$ & 217.78 \\
\hline $2.550 \mathrm{E}-02$ & 3.194 & $1.50 \mathrm{E}+01$ & 220.78 \\
\hline $3.200 \mathrm{E}-02$ & 3.793 & $1.60 \mathrm{E}+01$ & 223.78 \\
\hline $4.000 \mathrm{E}-02$ & 4.622 & $1.70 \mathrm{E}+01$ & 229.33 \\
\hline $5.250 \mathrm{E}-02$ & 5.587 & $1.80 \mathrm{E}+01$ & 236.61 \\
\hline \multirow[t]{2}{*}{$6.600 \mathrm{E}-02$} & 6.797 & $1.90 \mathrm{E}+01$ & 243.81 \\
\hline & & $2.00 \mathrm{E}+01$ & \\
\hline
\end{tabular}

\begin{tabular}{|c|c|c|c|}
\hline LOWER & CROSS & LOWER & CROSS \\
\hline $\begin{array}{l}\text { ENERGY } \\
(\mathrm{MEV})\end{array}$ & $\begin{array}{l}\text { SECTION } \\
(\mathrm{KEV}-\mathrm{B})\end{array}$ & $\begin{array}{l}\text { ENERGY } \\
(\mathrm{MEV})\end{array}$ & $\begin{array}{c}\text { SECTION } \\
(\mathrm{KEV}-\mathrm{B})\end{array}$ \\
\hline 1.000E-10 & 0.171 & $8.80 \mathrm{E}-02$ & 8.18 \\
\hline 1.000E-09 & 0.054 & $1.10 \mathrm{E}-01$ & 10.03 \\
\hline $1.000 \mathrm{E}-08$ & 0.028 & $1.35 \mathrm{E}-01$ & 10.90 \\
\hline $2.300 E-08$ & 0.019 & $1.60 \mathrm{E}-01$ & 12.09 \\
\hline $5.000 E-08$ & 0.014 & $1.90 \mathrm{E}-01$ & 13.20 \\
\hline $7.600 \mathrm{E}-08$ & 0.012 & $2.20 \mathrm{E}-01$ & 15.05 \\
\hline $1.150 \mathrm{E}-07$ & 0.009 & $2.55 E-01$ & 16.67 \\
\hline $1.700 \mathrm{E}-07$ & 0.008 & $2.90 E-01$ & 16.94 \\
\hline
\end{tabular}




$\begin{array}{lllr}2.550 \mathrm{E}-07 & 0.006 & 3.20 \mathrm{E}-01 & 19.28 \\ 3.800 \mathrm{E}-07 & 0.005 & 3.60 \mathrm{E}-01 & 18.41 \\ 5.500 \mathrm{E}-07 & 0.004 & 4.00 \mathrm{E}-01 & 16.72 \\ 8.400 \mathrm{E}-07 & 0.003 & 4.50 \mathrm{E}-01 & 14.82 \\ 1.275 \mathrm{E}-06 & 0.003 & 5.00 \mathrm{E}-01 & 20.14 \\ 1.900 \mathrm{E}-06 & 0.002 & 5.50 \mathrm{E}-01 & 22.73 \\ 2.800 \mathrm{E}-06 & 0.002 & 6.00 \mathrm{E}-01 & 25.03 \\ 4.250 \mathrm{E}-06 & 0.002 & 6.60 \mathrm{E}-01 & 25.74 \\ 6.300 \mathrm{E}-06 & 0.001 & 7.20 \mathrm{E}-01 & 28.57 \\ 9.200 \mathrm{E}-06 & 0.001 & 7.80 \mathrm{E}-01 & 32.97 \\ 1.350 \mathrm{E}-05 & 0.001 & 8.40 \mathrm{E}-01 & 30.58 \\ 2.100 \mathrm{E}-05 & 0.001 & 9.20 \mathrm{E}-01 & 31.16 \\ 3.000 \mathrm{E}-05 & 0.001 & 1.00 \mathrm{E}+00 & 34.64 \\ 4.500 \mathrm{E}-05 & 0.000 & 1.20 \mathrm{E}+00 & 41.37 \\ 6.900 \mathrm{E}-05 & 0.000 & 1.40 \mathrm{E}+00 & 45.41 \\ 1.000 \mathrm{E}-04 & 0.000 & 1.60 \mathrm{E}+00 & 57.42 \\ 1.350 \mathrm{E}-04 & 0.000 & 1.80 \mathrm{E}+00 & 58.45 \\ 1.700 \mathrm{E}-04 & 0.000 & 2.00 \mathrm{E}+00 & 72.95 \\ 2.200 \mathrm{E}-04 & 0.000 & 2.30 \mathrm{E}+00 & 85.63 \\ 2.800 \mathrm{E}-04 & 0.000 & 2.60 \mathrm{E}+00 & 96.38 \\ 3.600 \mathrm{E}-04 & 0.000 & 2.90 \mathrm{E}+00 & 107.51 \\ 4.500 \mathrm{E}-04 & 0.000 & 3.30 \mathrm{E}+00 & 109.38 \\ 5.750 \mathrm{E}-04 & 0.000 & 3.70 \mathrm{E}+00 & 113.26 \\ 7.600 \mathrm{E}-04 & 0.000 & 4.10 \mathrm{E}+00 & 114.63 \\ 9.600 \mathrm{E}-04 & 0.000 & 4.50 \mathrm{E}+00 & 114.19 \\ 1.275 \mathrm{E}-03 & 0.067 & 5.00 \mathrm{E}+00 & 114.07 \\ 1.600 \mathrm{E}-03 & 0.199 & 5.50 \mathrm{E}+00 & 115.57 \\ 2.000 \mathrm{E}-03 & 0.317 & 6.00 \mathrm{E}+00 & 120.64 \\ 2.700 \mathrm{E}-03 & 0.393 & 6.70 \mathrm{E}+00 & 125.40 \\ 3.400 \mathrm{E}-03 & 0.452 & 7.40 \mathrm{E}+00 & 137.41 \\ 4.500 \mathrm{E}-03 & 0.535 & 8.20 \mathrm{E}+00 & 152.24 \\ 5.500 \mathrm{E}-03 & 0.656 & 9.00 \mathrm{E}+00 & 162.51 \\ 7.200 \mathrm{E}-03 & 0.839 & 1.00 \mathrm{E}+01 & 172.96 \\ 9.200 \mathrm{E}-03 & 1.041 & 1.10 \mathrm{E}+01 & 185.64 \\ 1.200 \mathrm{E}-02 & 1.295 & 1.20 \mathrm{E}+01 & 193.26 \\ 1.500 \mathrm{E}-02 & 1.617 & 1.30 \mathrm{E}+01 & 197.94 \\ 1.900 \mathrm{E}-02 & 2.005 & 1.40 \mathrm{E}+01 & 203.50 \\ 2.550 \mathrm{E}-02 & 2.416 & 1.50 \mathrm{E}+01 & 211.43 \\ 3.200 \mathrm{E}-02 & 2.994 & 1.60 \mathrm{E}+01 & 226.43 \\ 4.000 \mathrm{E}-02 & 4.962 & 1.70 \mathrm{E}+01 & 233.30 \\ 5.250 \mathrm{E}-02 & 5.285 & 1.80 \mathrm{E}+01 & 234.80 \\ 6.600 \mathrm{E}-02 & 7.764 & 1.90 \mathrm{E}+01 & 244.89 \\ & & 2.00 \mathrm{E}+01 & \end{array}$

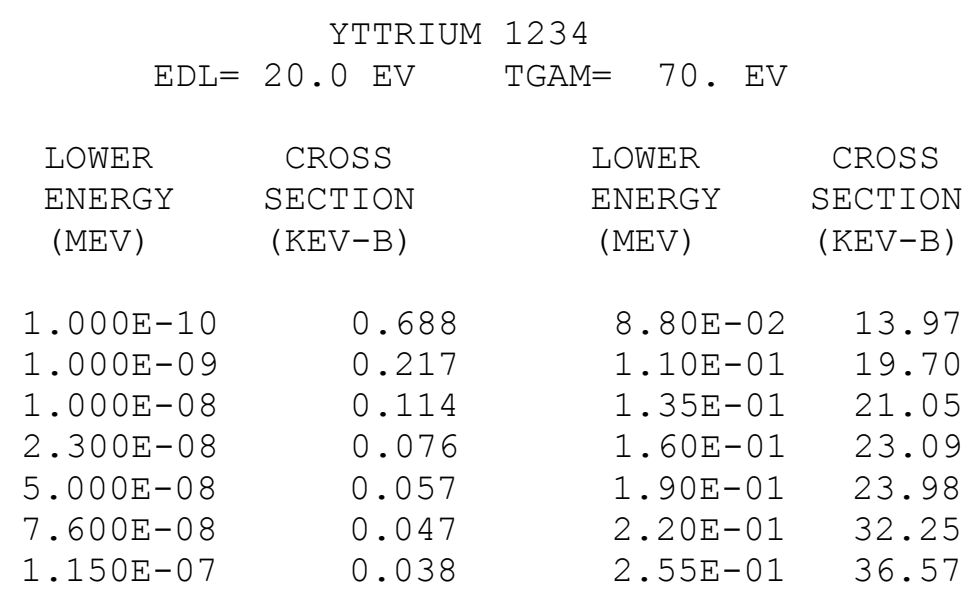




$\begin{array}{lrrr}1.700 \mathrm{E}-07 & 0.031 & 2.90 \mathrm{E}-01 & 41.23 \\ 2.550 \mathrm{E}-07 & 0.026 & 3.20 \mathrm{E}-01 & 38.28 \\ 3.800 \mathrm{E}-07 & 0.021 & 3.60 \mathrm{E}-01 & 48.01 \\ 5.500 \mathrm{E}-07 & 0.017 & 4.00 \mathrm{E}-01 & 51.42 \\ 8.400 \mathrm{E}-07 & 0.014 & 4.50 \mathrm{E}-01 & 53.07 \\ 1.275 \mathrm{E}-06 & 0.011 & 5.00 \mathrm{E}-01 & 60.84 \\ 1.900 \mathrm{E}-06 & 0.009 & 5.50 \mathrm{E}-01 & 56.70 \\ 2.800 \mathrm{E}-06 & 0.008 & 6.00 \mathrm{E}-01 & 59.75 \\ 4.250 \mathrm{E}-06 & 0.006 & 6.60 \mathrm{E}-01 & 63.24 \\ 6.300 \mathrm{E}-06 & 0.005 & 7.20 \mathrm{E}-01 & 64.78 \\ 9.200 \mathrm{E}-06 & 0.004 & 7.80 \mathrm{E}-01 & 66.35 \\ 1.350 \mathrm{E}-05 & 0.003 & 8.40 \mathrm{E}-01 & 67.82 \\ 2.100 \mathrm{E}-05 & 0.003 & 9.20 \mathrm{E}-01 & 69.19 \\ 3.000 \mathrm{E}-05 & 0.002 & 1.00 \mathrm{E}+00 & 72.40 \\ 4.500 \mathrm{E}-05 & 0.002 & 1.20 \mathrm{E}+00 & 77.68 \\ 6.900 \mathrm{E}-05 & 0.002 & 1.40 \mathrm{E}+00 & 82.66 \\ 1.000 \mathrm{E}-04 & 0.001 & 1.60 \mathrm{E}+00 & 86.15 \\ 1.350 \mathrm{E}-04 & 0.001 & 1.80 \mathrm{E}+00 & 89.03 \\ 1.700 \mathrm{E}-04 & 0.001 & 2.00 \mathrm{E}+00 & 95.33 \\ 2.200 \mathrm{E}-04 & 0.001 & 2.30 \mathrm{E}+00 & 103.18 \\ 2.800 \mathrm{E}-04 & 0.001 & 2.60 \mathrm{E}+00 & 111.19 \\ 3.600 \mathrm{E}-04 & 0.001 & 2.90 \mathrm{E}+00 & 118.50 \\ 4.500 \mathrm{E}-04 & 0.001 & 3.30 \mathrm{E}+00 & 124.59 \\ 5.750 \mathrm{E}-04 & 0.001 & 3.70 \mathrm{E}+00 & 129.99 \\ 7.600 \mathrm{E}-04 & 0.001 & 4.10 \mathrm{E}+00 & 136.39 \\ 9.600 \mathrm{E}-04 & 0.135 & 4.50 \mathrm{E}+00 & 143.48 \\ 1.275 \mathrm{E}-03 & 0.260 & 5.00 \mathrm{E}+00 & 152.06 \\ 1.600 \mathrm{E}-03 & 0.347 & 5.50 \mathrm{E}+00 & 160.54 \\ 2.000 \mathrm{E}-03 & 0.641 & 6.00 \mathrm{E}+00 & 170.63 \\ 2.700 \mathrm{E}-03 & 0.523 & 6.70 \mathrm{E}+00 & 180.74 \\ 3.400 \mathrm{E}-03 & 0.574 & 7.40 \mathrm{E}+00 & 189.96 \\ 4.500 \mathrm{E}-03 & 0.690 & 8.20 \mathrm{E}+00 & 197.17 \\ 5.500 \mathrm{E}-03 & 0.791 & 9.00 \mathrm{E}+00 & 203.39 \\ 7.200 \mathrm{E}-03 & 1.867 & 1.00 \mathrm{E}+01 & 209.41 \\ 9.200 \mathrm{E}-03 & 2.088 & 1.10 \mathrm{E}+01 & 217.32 \\ 1.200 \mathrm{E}-02 & 2.139 & 1.20 \mathrm{E}+01 & 225.01 \\ 1.500 \mathrm{E}-02 & 2.938 & 1.30 \mathrm{E}+01 & 236.20 \\ 1.900 \mathrm{E}-02 & 3.860 & 1.40 \mathrm{E}+01 & 249.88 \\ 2.550 \mathrm{E}-02 & 5.011 & 1.50 \mathrm{E}+01 & 264.66 \\ 3.200 \mathrm{E}-02 & 5.709 & 1.60 \mathrm{E}+01 & 279.64 \\ 4.000 \mathrm{E}-02 & 6.449 & 1.70 \mathrm{E}+01 & 295.33 \\ 5.250 \mathrm{E}-02 & 11.082 & 1.80 \mathrm{E}+01 & 309.92 \\ 6.600 \mathrm{E}-02 & 13.203 & 1.90 \mathrm{E}+01 & 324.31 \\ & & 2.00 \mathrm{E}+01 & \end{array}$

BARIUM

\begin{tabular}{lccr}
\multicolumn{2}{c}{ EDL $=20.0 \mathrm{EV}$} & TGAM $=$ 41. EV \\
$\begin{array}{l}\text { LOWER } \\
\begin{array}{l}\text { ENERGY } \\
(\mathrm{MEV})\end{array}\end{array}$ & $\begin{array}{c}\text { CROSS } \\
\text { (KETION }\end{array}$ & $\begin{array}{l}\text { LOWER } \\
\text { ENERGY } \\
(\mathrm{MEV})\end{array}$ & $\begin{array}{c}\text { CROSS } \\
\text { SECTION } \\
(\mathrm{KEV}-\mathrm{B})\end{array}$ \\
$1.000 \mathrm{E}-10$ & 0.354 & $8.80 \mathrm{E}-02$ & 7.91 \\
$1.000 \mathrm{E}-09$ & 0.112 & $1.10 \mathrm{E}-01$ & 11.06 \\
$1.000 \mathrm{E}-08$ & 0.059 & $1.35 \mathrm{E}-01$ & 11.56 \\
$2.300 \mathrm{E}-08$ & 0.039 & $1.60 \mathrm{E}-01$ & 11.82 \\
$5.000 \mathrm{E}-08$ & 0.030 & $1.90 \mathrm{E}-01$ & 12.96 \\
$7.600 \mathrm{E}-08$ & 0.024 & $2.20 \mathrm{E}-01$ & 13.14
\end{tabular}




\begin{tabular}{|c|c|c|c|}
\hline $1.150 \mathrm{E}-07$ & 0.020 & $2.55 E-01$ & 14.89 \\
\hline $1.700 E-07$ & 0.016 & $2.90 \mathrm{E}-01$ & 16.42 \\
\hline $2.550 E-07$ & 0.013 & $3.20 E-01$ & 17.69 \\
\hline $3.800 E-07$ & 0.011 & $3.60 E-01$ & 18.47 \\
\hline $5.500 \mathrm{E}-07$ & 0.009 & $4.00 \mathrm{E}-01$ & 19.98 \\
\hline $8.400 \mathrm{E}-07$ & 0.007 & $4.50 \mathrm{E}-01$ & 22.22 \\
\hline $1.275 E-06$ & 0.006 & $5.00 \mathrm{E}-01$ & 25.58 \\
\hline $1.900 \mathrm{E}-06$ & 0.005 & $5.50 \mathrm{E}-01$ & 28.58 \\
\hline $2.800 \mathrm{E}-06$ & 0.004 & $6.00 \mathrm{E}-01$ & 31.35 \\
\hline $4.250 E-06$ & 0.003 & $6.60 \mathrm{E}-01$ & 35.56 \\
\hline $6.300 E-06$ & 0.003 & $7.20 \mathrm{E}-01$ & 38.91 \\
\hline $9.200 E-06$ & 0.003 & $7.80 E-01$ & 41.96 \\
\hline $1.350 \mathrm{E}-05$ & 0.002 & $8.40 \mathrm{E}-01$ & 46.74 \\
\hline $2.100 \mathrm{E}-05$ & 0.002 & $9.20 \mathrm{E}-01$ & 51.59 \\
\hline $3.000 \mathrm{E}-05$ & 0.002 & $1.00 \mathrm{E}+00$ & 61.03 \\
\hline $4.500 \mathrm{E}-05$ & 0.002 & $1.20 \mathrm{E}+00$ & 72.09 \\
\hline $6.900 E-05$ & 0.001 & $1.40 \mathrm{E}+00$ & 80.80 \\
\hline $1.000 \mathrm{E}-04$ & 0.001 & $1.60 \mathrm{E}+00$ & 88.17 \\
\hline $1.350 E-04$ & 0.001 & $1.80 \mathrm{E}+00$ & 92.50 \\
\hline $1.700 \mathrm{E}-04$ & 0.001 & $2.00 \mathrm{E}+00$ & 96.51 \\
\hline $2.200 E-04$ & 0.001 & $2.30 \mathrm{E}+00$ & 102.67 \\
\hline $2.800 \mathrm{E}-04$ & 0.001 & $2.60 E+00$ & 106.47 \\
\hline $3.600 E-04$ & 0.001 & $2.90 \mathrm{E}+00$ & 109.06 \\
\hline $4.500 E-04$ & 0.011 & $3.30 E+00$ & 111.06 \\
\hline $5.750 E-04$ & 0.014 & $3.70 E+00$ & 112.86 \\
\hline $7.600 \mathrm{E}-04$ & 0.011 & $4.10 \mathrm{E}+00$ & 118.75 \\
\hline $9.600 \mathrm{E}-04$ & 0.010 & $4.50 \mathrm{E}+00$ & 126.25 \\
\hline $1.275 \mathrm{E}-03$ & 0.026 & $5.00 \mathrm{E}+00$ & 135.15 \\
\hline $1.600 \mathrm{E}-03$ & 0.149 & $5.50 \mathrm{E}+00$ & 144.84 \\
\hline $2.000 \mathrm{E}-03$ & 0.336 & $6.00 \mathrm{E}+00$ & 155.44 \\
\hline $2.700 \mathrm{E}-03$ & 0.435 & $6.70 \mathrm{E}+00$ & 167.54 \\
\hline $3.400 \mathrm{E}-03$ & 0.526 & $7.40 \mathrm{E}+00$ & 184.45 \\
\hline $4.500 \mathrm{E}-03$ & 0.606 & $8.20 E+00$ & 201.75 \\
\hline $5.500 \mathrm{E}-03$ & 0.709 & $9.00 \mathrm{E}+00$ & 219.46 \\
\hline $7.200 \mathrm{E}-03$ & 0.955 & $1.00 \mathrm{E}+01$ & 242.27 \\
\hline $9.200 \mathrm{E}-03$ & 0.958 & $1.10 \mathrm{E}+01$ & 263.58 \\
\hline $1.200 \mathrm{E}-02$ & 1.165 & $1.20 \mathrm{E}+01$ & 283.29 \\
\hline $1.500 \mathrm{E}-02$ & 1.418 & $1.30 \mathrm{E}+01$ & 301.61 \\
\hline $1.900 \mathrm{E}-02$ & 1.954 & $1.40 \mathrm{E}+01$ & 326.43 \\
\hline $2.550 \mathrm{E}-02$ & 2.887 & $1.50 \mathrm{E}+01$ & 347.05 \\
\hline $3.200 \mathrm{E}-02$ & 3.122 & $1.60 \mathrm{E}+01$ & 372.17 \\
\hline $4.000 \mathrm{E}-02$ & 4.069 & $1.70 \mathrm{E}+01$ & 391.19 \\
\hline $5.250 \mathrm{E}-02$ & 4.876 & $1.80 \mathrm{E}+01$ & 411.51 \\
\hline $6.600 \mathrm{E}-02$ & 6.389 & 1.90E+01 & 428.52 \\
\hline
\end{tabular}

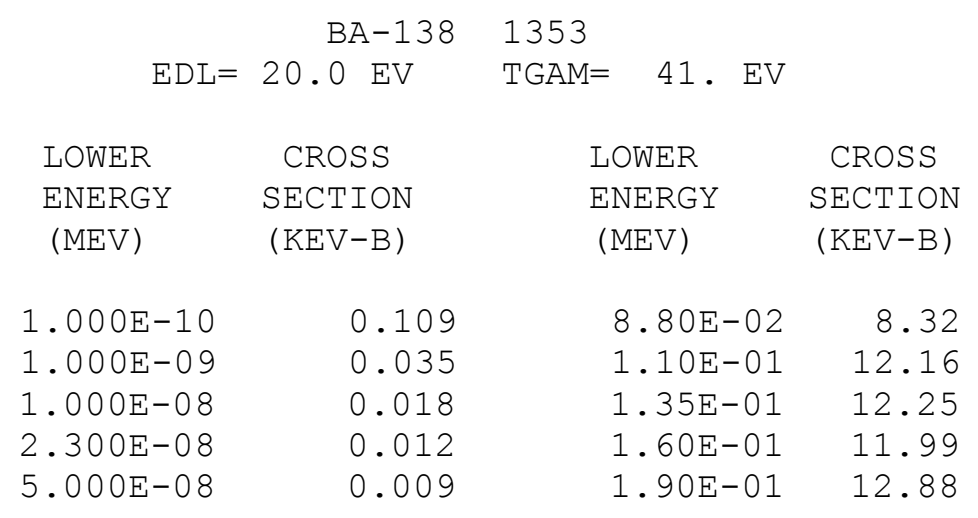




$\begin{array}{lllr}7.600 \mathrm{E}-08 & 0.007 & 2.20 \mathrm{E}-01 & 12.33 \\ 1.150 \mathrm{E}-07 & 0.006 & 2.55 \mathrm{E}-01 & 13.90 \\ 1.700 \mathrm{E}-07 & 0.005 & 2.90 \mathrm{E}-01 & 15.24 \\ 2.550 \mathrm{E}-07 & 0.004 & 3.20 \mathrm{E}-01 & 16.14 \\ 3.800 \mathrm{E}-07 & 0.003 & 3.60 \mathrm{E}-01 & 16.22 \\ 5.500 \mathrm{E}-07 & 0.003 & 4.00 \mathrm{E}-01 & 17.08 \\ 8.400 \mathrm{E}-07 & 0.002 & 4.50 \mathrm{E}-01 & 18.80 \\ 1.275 \mathrm{E}-06 & 0.002 & 5.00 \mathrm{E}-01 & 22.07 \\ 1.900 \mathrm{E}-06 & 0.002 & 5.50 \mathrm{E}-01 & 24.81 \\ 2.800 \mathrm{E}-06 & 0.001 & 6.00 \mathrm{E}-01 & 27.12 \\ 4.250 \mathrm{E}-06 & 0.001 & 6.60 \mathrm{E}-01 & 31.29 \\ 6.300 \mathrm{E}-06 & 0.001 & 7.20 \mathrm{E}-01 & 34.17 \\ 9.200 \mathrm{E}-06 & 0.001 & 7.80 \mathrm{E}-01 & 36.62 \\ 1.350 \mathrm{E}-05 & 0.001 & 8.40 \mathrm{E}-01 & 41.23 \\ 2.100 \mathrm{E}-05 & 0.001 & 9.20 \mathrm{E}-01 & 45.62 \\ 3.000 \mathrm{E}-05 & 0.001 & 1.00 \mathrm{E}+00 & 55.00 \\ 4.500 \mathrm{E}-05 & 0.001 & 1.20 \mathrm{E}+00 & 65.28 \\ 6.900 \mathrm{E}-05 & 0.001 & 1.40 \mathrm{E}+00 & 72.31 \\ 1.000 \mathrm{E}-04 & 0.001 & 1.60 \mathrm{E}+00 & 77.96 \\ 1.350 \mathrm{E}-04 & 0.001 & 1.80 \mathrm{E}+00 & 79.85 \\ 1.700 \mathrm{E}-04 & 0.001 & 2.00 \mathrm{E}+00 & 81.53 \\ 2.200 \mathrm{E}-04 & 0.001 & 2.30 \mathrm{E}+00 & 86.66 \\ 2.800 \mathrm{E}-04 & 0.001 & 2.60 \mathrm{E}+00 & 88.42 \\ 3.600 \mathrm{E}-04 & 0.001 & 2.90 \mathrm{E}+00 & 88.16 \\ 4.500 \mathrm{E}-04 & 0.001 & 3.30 \mathrm{E}+00 & 87.96 \\ 5.750 \mathrm{E}-04 & 0.001 & 3.70 \mathrm{E}+00 & 87.84 \\ 7.600 \mathrm{E}-04 & 0.001 & 4.10 \mathrm{E}+00 & 93.80 \\ 9.600 \mathrm{E}-04 & 0.001 & 4.50 \mathrm{E}+00 & 102.21 \\ 1.275 \mathrm{E}-03 & 0.018 & 5.00 \mathrm{E}+00 & 111.51 \\ 1.600 \mathrm{E}-03 & 0.158 & 5.50 \mathrm{E}+00 & 121.22 \\ 2.000 \mathrm{E}-03 & 0.284 & 6.00 \mathrm{E}+00 & 131.72 \\ 2.700 \mathrm{E}-03 & 0.368 & 6.70 \mathrm{E}+00 & 142.72 \\ 3.400 \mathrm{E}-03 & 0.424 & 7.40 \mathrm{E}+00 & 154.63 \\ 4.500 \mathrm{E}-03 & 0.505 & 8.20 \mathrm{E}+00 & 165.03 \\ 5.500 \mathrm{E}-03 & 0.608 & 9.00 \mathrm{E}+00 & 173.83 \\ 7.200 \mathrm{E}-03 & 0.894 & 1.00 \mathrm{E}+01 & 187.34 \\ 9.200 \mathrm{E}-03 & 0.830 & 1.10 \mathrm{E}+01 & 197.85 \\ 1.200 \mathrm{E}-02 & 1.032 & 1.20 \mathrm{E}+01 & 206.56 \\ 1.500 \mathrm{E}-02 & 1.285 & 1.30 \mathrm{E}+01 & 213.97 \\ 1.900 \mathrm{E}-02 & 1.888 & 1.40 \mathrm{E}+01 & 230.19 \\ 2.550 \mathrm{E}-02 & 3.008 & 1.50 \mathrm{E}+01 & 240.50 \\ 3.200 \mathrm{E}-02 & 3.150 & 1.60 \mathrm{E}+01 & 257.02 \\ 4.000 \mathrm{E}-02 & 4.219 & 1.70 \mathrm{E}+01 & 265.04 \\ 5.250 \mathrm{E}-02 & 5.027 & 1.80 \mathrm{E}+01 & 274.66 \\ 6.600 \mathrm{E}-02 & 6.709 & 1.90 \mathrm{E}+01 & 284.16 \\ & & 2.00 \mathrm{E}+01 & \\ & & & \end{array}$




\section{Appendix B: ATR Power History for Cycle 153B}

\section{INTEROFFICE MEMORANDUM}

Date:

April 22, 2013

To:

R. A. Jordan

From:

D. E. Hale<smiles>C(NC1CC1)=C1CC1</smiles>

Subject: ADVANCED TEST REACTOR (ATR) POWER HISTORY THROUGH CYCLE $153 \mathrm{~A} / \mathrm{B}-1$

References: (a) A. V. Briscoe letter to J. L. Durney, AVB-9-77, ATR Power History Through Cycle 34C-1, June 7, 1977

(b) C. C. Swanson letter to J. L. Dumey, CAS-05-86, ATR Power History Through Cycle 72A-1, February 3, 1986

(c) L. S. Loret letter to E. C. Anderson, Sr., LSL-11-94, ATR Power History Through Cycle 102B-1, February 28, 1994

(d) D. E. Hale letter to J. C. Chapman, DEH-05-04, Advanced Test Reactor (ATR) Power History Through Cycle 133B-1, August 18, 2004

Table 1 lists the ATR N-16 constrained power history data since the Beryllium VI Core Internals Changeout (Cycle 134A-1) through Cycle 153A/B-1. The ATR power history prior to Cycle 134A-I is presented in the references.

Table 2 lists the accumulated $\mathrm{N}-16$ total lobe MWd and total core MWd as obtained from the ATR DAS for Cycle 134A-1 through 153A/B-1.

\section{DEH}

cc: J. O. Brower, MS 3407

G. S. Chang, MS 3870

C. D. Cooper, MS 3407

C. A. Dahl, MS 3818

M. A. David, MS 7136

G. W. Davis, MS 7117

K. R. Estes, MS 7130

R. L. Fulks, MS 7130

J. E. Giebel, MS 7104

G. C. Hawkley, MS 7136

R. Holtz, MS 7136

R. C. Howard, MS 7101

C. D. Jackson, MS 7106

C. C. Jensen, MS 7113 
R. A. Jordan

April 22, 2013

Page 2

W. F. Jones, MS 3818

T. L. Julius, MS 7104

V. C. Kirkpatrick, MS 7106

A. W. LaPorta, MS 7136 h

M. A. Lillo, MS 3870

S. G. Louk, MS 7111

M. D. Love, MS 7117

Z. S. Miller, MS 7136

M. K. Morrison, MS 7136

R. K. Murray, MS 7111

D. Ogden, MS 3818

J. R. Peters, MS 7103

P. A. Roth, MS 3425

D. J. Schoonen, MS 3870

C. J. Stanley, MS 7136

M. E. Stengel (2), MS 7103

C. R. Tyler, MtyS 3835

D. J. Utterbeck, MS 3870

J. F. Williams, MS 7101

CSAP Surveillance File

D. E. Hale Letter File (DEH-02-13)

Uniform File Code: $\underline{8153}$

Disposition Authority: A17-32-b-1

Retention Schedule: Destroy when 6 years old

NOTE: Original disposition authority, retention schedule, and Uniform Filing Code applied by the sender may not be appropriate for all recipients. Make adjustments as needed. 
TABLE 1

SUMMARY OF ATR POWER HISTORY

\begin{tabular}{|c|c|c|c|c|c|c|c|c|c|c|c|c|}
\hline \multirow{2}{*}{$\begin{array}{c}\text { Cycle } \\
\text { No. } \\
\end{array}$} & \multicolumn{5}{|c|}{$\mathrm{N}-16$ Average Lobe Powers (MM) } & \multicolumn{6}{|c|}{$\mathrm{N}-16$ Lobe MWd } & \multirow[b]{2}{*}{ EFPD } \\
\hline & $\underline{N W}$ & NE & $\mathrm{c}$ & SW & $\underline{\text { SE }}$ & NW & $\underline{\mathrm{NE}}$ & $\mathrm{C}$ & $\underline{\text { SW }}$ & SE & Total & \\
\hline $134 \mathrm{~A}-1$ & $\ldots$ & - & $\ldots$ & - & -... & 0 & 0 & 0 & 0 & 0 & 0 & $\ldots$ \\
\hline $134 \mathrm{~A}-2$ & $\ldots$ & - & .... & - & ... & $\frac{0.2}{0.2}$ & $\frac{0.3}{0.3}$ & $\frac{0.4}{0.4}$ & $\frac{0.4}{0.4}$ & $\frac{0.4}{0.4}$ & $\frac{2.0}{2.0}$ & $\ldots$ \\
\hline $1348-1$ & 18.0 & 18.0 & 24.5 & 23.0 & 25.0 & 452.8 & 452.2 & 615.5 & 578.5 & 628.6 & 2727,6 & 25.1 \\
\hline 1348-2 & 18.0 & 18.0 & 25.8 & 23.0 & 25.0 & $\frac{385.6}{838.4}$ & $\frac{385.8}{838.0}$ & $\frac{553.5}{1169.0}$ & $\frac{493.2}{1071.7}$ & $\frac{536.1}{1164.7}$ & $\frac{2354.2}{5081.8}$ & $\frac{21.4}{46.5}$ \\
\hline $135 \mathrm{~A}-1$ & 18.0 & 18.0 & 29.8 & 28.1 & 35.4 & 236.2 & 236.0 & 391.1 & 368.6 & 464.0 & 1695.8 & 13.1 \\
\hline $135 \mathrm{~B}-1$ & 18.0 & 18.0 & 24.8 & 23.0 & 25.0 & 458.3 & 458.4 & 630.8 & 585.3 & 636.5 & 2769.2 & 25.5 \\
\hline $1358-2$ & 18.0 & 18.0 & 25.2 & 23.0 & 25.0 & $\frac{405.0}{863.3}$ & $\frac{405.7}{864.1}$ & $\frac{567.4}{1198.2}$ & $\frac{517.8}{1103.1}$ & $\frac{563.0}{1190.5}$ & $\frac{2458.9}{5228.1}$ & $\frac{22.5}{48.0}$ \\
\hline $135 \mathrm{C}-1$ & 18.0 & 18.0 & 25.0 & 23.0 & 25.0 & 729.9 & 729.7 & 1013.5 & 933.0 & 1013.9 & 4419.9 & 40.6 \\
\hline $136 \mathrm{~A}-1$ & 18.0 & 18.0 & 24.0 & 23.0 & 23.0 & 916.2 & 916.4 & 1218.9 & 1169.5 & 1170.2 & 5391.1 & 50.9 \\
\hline $136 \mathrm{~B}-1$ & 18.0 & 18.0 & 23.9 & 23.0 & 23.0 & 701.9 & 702.3 & 931.2 & 896.9 & 897.0 & 4129.4 & 39.0 \\
\hline 137A-1 & 18.0 & 18.0 & 24.7 & 20.0 & 25.0 & 975.4 & 974.8 & 1336.2 & 1083.2 & 1353.3 & 5722.8 & 54.1 \\
\hline $1378-1$ & 20.0 & 17.9 & 35.5 & 56.6 & 30.4 & 242.0 & 217.0 & 429.6 & 685.4 & 367.6 & 1941.6 & 12.1 \\
\hline $138 \mathrm{~A}-1$ & 18.0 & 18.0 & 23.6 & 23.0 & 25.0 & 1046.9 & 1047.7 & 1370.7 & 1336.4 & 1453.5 & 6255.1 & 58.1 \\
\hline $1388-1$ & 18.0 & 18.0 & 23.3 & 23.0 & 25.0 & 838.5 & 839.6 & 1084.8 & 1070.9 & 1164.6 & 4998.5 & 46.6 \\
\hline 139A-1 & 18.0 & 18.0 & 23.9 & 23.0 & 25.0 & 928.7 & 929.1 & 1231.1 & 1186.3 & 1289.5 & 5564.6 & 51,6 \\
\hline $1390-1$ & 18.0 & 18.0 & 23.2 & 23.0 & 23.0 & 919.7 & 919.7 & 1187.5 & 1174,9 & 11750 & 5376.8 & 51,1 \\
\hline $140 \mathrm{~A}=1$ & 18.0 & 18.0 & 21.8 & 23.0 & 23.0 & 837.0 & 837.2 & 1012.9 & 1069.7 & 1069.4 & 4826.1 & 46.5 \\
\hline $1408-1$ & 18.0 & 17.7 & 21.8 & 23.6 & 23.0 & 641.7 & 629.5 & 777.2 & 842.9 & 820.0 & 3711.3 & 35.7 \\
\hline $141 \mathrm{~A}-1$ & 18.0 & 18.0 & 23.4 & 23.0 & 23.0 & 583.3 & 583.1 & 756.8 & 745.1 & 745.5 & 3413.7 & 32.4 \\
\hline $142 \mathrm{~A}-1$ & 23.0 & 18.0 & 24.7 & 24.8 & 23.0 & 1104.9 & 864.8 & 1186.0 & 1192.5 & 1104.0 & 5452.2 & 48.0 \\
\hline $1428-1$ & 23.0 & 18.0 & 25.4 & 25.4 & 25.0 & 1196.9 & 936.7 & 1323.4 & 1322.5 & 1298.7 & 6078.2 & 52.0 \\
\hline $143 \mathrm{~A}-1 / 2$ & 18.0 & 18.0 & 24.3 & 26.9 & 25.0 & 880.0 & 882.5 & 1187.7 & 1315.4 & 1223.1 & 5488.7 & 48.9 \\
\hline $1438-1$ & 18.0 & 18.0 & 24.9 & 27.0 & 25.1 & 1032.1 & 1032.6 & 1423.5 & 1543.7 & 1435.0 & 6466.9 & 57.3 \\
\hline $144 \mathrm{~A}-1$ & 18.0 & 18.0 & 23.1 & 23.0 & 25.1 & 787.0 & 787.0 & 1006.7 & 1004.5 & 1093.4 & 4678.6 & 43.7 \\
\hline $1448-1$ & 18.0 & 18.1 & 22.4 & 23.0 & 23.0 & 932.3 & 933.4 & 1155.7 & 1190.7 & 1190.9 & 5403.0 & 51.7 \\
\hline
\end{tabular}


Page 2 of 2

TABLE 1

SUMMARY OF ATR POWER HISTORY

\begin{tabular}{|c|c|c|c|c|c|c|c|c|c|c|c|c|}
\hline \multirow{2}{*}{$\begin{array}{c}\text { Cycle } \\
\text { No. } \\
\end{array}$} & \multicolumn{5}{|c|}{ N-16 Average Lobe Powers (MW) } & \multicolumn{6}{|c|}{ N-16 Lobe MWd } & \multirow[b]{2}{*}{ EFPD } \\
\hline & $\underline{\mathrm{NW}}$ & NE & C & sW & SE & NW & NE & $c$ & $\underline{s w}$ & SE & Iotal & \\
\hline $145 \mathrm{~A}-1$ & 18.0 & 17.9 & 23.2 & 23.8 & 25.7 & 983.0 & 980.9 & 1267.3 & 1299.5 & 1407.8 & 5938.4 & 54.7 \\
\hline $1458-1$ & 17.8 & 17.8 & 23.0 & 24.6 & 25.8 & 1020.5 & 1020.0 & 1321.4 & 1407.8 & 1478.3 & 6247.9 & 57.3 \\
\hline $146 \mathrm{~A}-1$ & 18.0 & 18.0 & 24.3 & 25.8 & 26.0 & 906.8 & 906.8 & 1225.7 & 1300.0 & 1312.6 & 5651.9 & 50.5 \\
\hline $1468-1$ & 23.0 & 18.0 & 26.0 & 23.0 & 26.0 & 903.7 & 707.1 & 1021.6 & 903.9 & 1021.0 & 45572 & 39.2 \\
\hline $147 \mathrm{~A}-1$ & 23.0 & 18.0 & 24.1 & 20.9 & 23.0 & 1156.9 & 904.4 & 1208.4 & 1049.4 & 1155.2 & 5474.3 & 50.2 \\
\hline $148 \mathrm{~A}-1$ & 18.0 & 18.0 & 23.6 & 22.0 & 23.0 & 856.0 & 855.8 & 1121.4 & 1043.8 & 1093.6 & 4970.6 & 47.5 \\
\hline 1488-1 & 18.0 & 18.0 & 23.0 & 23.8 & 23.0 & 927.5 & 926.7 & 1181.6 & 1224.0 & 1185.0 & 5444.8 & 51.5 \\
\hline $149 \mathrm{~A}-1$ & 18.0 & 18.0 & 24.2 & 24.0 & 23.0 & 662.5 & 6627 & 891.3 & 883.3 & 846.8 & 3946.5 & 36.8 \\
\hline $149 B-1$ & 18.0 & 18.0 & 242 & 23.0 & 23.0 & 964.4 & 964.5 & 1297.8 & 1231.6 & 1230.8 & 5689.0 & 53.6 \\
\hline $150 \mathrm{~A}-1$ & 18.9 & 18.0 & 30.5 & 37.5 & 35.1 & 233.4 & 221.6 & 375.9 & 462.7 & 432.6 & 1726.2 & 12.3 \\
\hline 150B-1 & 19.9 & 18.0 & 24.2 & 230 & 23.1 & 832.8 & 754.7 & 1014.6 & 964.9 & 966.0 & 4533.0 & 41.9 \\
\hline $151 \mathrm{~A}=1$ & 18.9 & 14.2 & 22.0 & 23.6 & 23.0 & 1058.6 & 800.0 & 1237.0 & 1324.4 & 1289.0 & 5709.0 & 56.1 \\
\hline $151 \mathrm{~B}-1 / 2$ & 18.9 & 14.5 & 22.1 & 23.0 & 23.0 & 971.4 & 741.8 & 1134.9 & 1181.9 & 1180.0 & 5209.9 & 51.3 \\
\hline $152 \mathrm{~A}-1 / 6$ & - & $\cdots$ & - & $\ldots$ & - & 0.3 & 0.3 & 0.5 & 0.4 & 0.5 & 2 & - \\
\hline $152 \mathrm{~B}-1$ & 18.9 & 15.9 & 22.4 & 23.0 & 23.0 & 966.4 & 813.0 & 1141.3 & 1172.1 & 1173.6 & 5266.4 & 51.0 \\
\hline 153A/B-1 & 19.7 & 19.7 & 30.8 & 35.4 & 44.0 & 265.2 & 265.4 & 414.5 & 476.1 & 591.4 & 2012.6 & 13.45 \\
\hline
\end{tabular}


Page 1 of 2

TABLE 2

SUMMARY OF ACCUMULATED N-16 MWd

\begin{tabular}{|c|c|c|c|c|c|c|}
\hline \multirow{2}{*}{$\begin{array}{c}\text { Cycle } \\
\text { No }\end{array}$} & \multicolumn{5}{|c|}{ Lobe } & \multirow{2}{*}{$\begin{array}{l}\text { Total } \\
\text { MWd }\end{array}$} \\
\hline & NW & $\mathrm{NE}$ & $\mathrm{C}$ & SW & $\mathrm{SE}$ & \\
\hline $134 \mathrm{~A}-1$ & 0 & 0 & 0 & 0 & 0 & 0 \\
\hline $134 \mathrm{~A}-2$ & 0.2 & 0.3 & 0.4 & 0.4 & 0.4 & 2.0 \\
\hline $134 \mathrm{~B}-1$ & 453.0 & 452.5 & 615.9 & 578,9 & 629.0 & 2729.6 \\
\hline $134 \mathrm{~B}-2$ & 838.6 & 838.3 & 1169.4 & 1072.1 & 1165.1 & 5083.8 \\
\hline $135 \mathrm{~A}-1$ & 1074.8 & 1074.3 & 1560.4 & 1440.8 & 1629.0 & 6779.7 \\
\hline $1358-1$ & 1533.1 & 1532.7 & 2191.2 & 2026.0 & 2265.5 & 9548.9 \\
\hline $1358-2$ & 1938.1 & 1938.4 & 2758.6 & 2543.8 & 2828.5 & 12007.8 \\
\hline $135 \mathrm{C}-1$ & 2668.0 & 2668.1 & 3772.1 & 3476.8 & 3842.4 & 16427.7 \\
\hline $136 \mathrm{~A}-1$ & 3584.2 & 3584.5 & 4991.0 & 4646.3 & 5012.6 & 21818.8 \\
\hline $136 \mathrm{~B}-1$ & 4286.1 & 4286.8 & 5922.2 & 5543.2 & 5909.5 & 25948.2 \\
\hline $137 \mathrm{~A}-1$ & 5261.5 & 5261.6 & 7258.3 & 6626.4 & 7262.8 & 31670.9 \\
\hline $1378-1$ & 5503.5 & 5478.6 & 7687.9 & 7311.8 & 7630.5 & 336125 \\
\hline $138 \mathrm{~A}-1$ & 6550.3 & 6526.3 & 9058.6 & 8648.2 & 9083.9 & 39867.7 \\
\hline $138 \mathrm{~B}-1$ & 7388.9 & 7365.9 & 10143.4 & 9719.2 & 10248.6 & 44866.2 \\
\hline $139 \mathrm{~A}-1$ & 8317.6 & 8295.0 & 11374.5 & 10905.4 & 11538.1 & 50430.8 \\
\hline $1398-1$ & 9237.3 & 9214.6 & 12562.0 & 12080.3 & 12713.1 & 55807.6 \\
\hline $140 \mathrm{~A}-1$ & 10074.3 & 10051.8 & 13574.9 & 13150.0 & 13782.5 & 60633.7 \\
\hline $140 \mathrm{~B}-1$ & 10716.0 & 10681.3 & 14352.1 & 13992.9 & 14602.5 & 64345.0 \\
\hline $141 \mathrm{~A}-1$ & 11299.3 & 11264.4 & 15108.9 & 14737.9 & 15347.9 & 67758.6 \\
\hline $142 \mathrm{~A}-1$ & 12404.2 & 12129.1 & 16294.9 & 15930.4 & 16452.0 & 73210.9 \\
\hline $142 \mathrm{~B}-1$ & 13601.1 & 13065.8 & 17618.4 & 17252.8 & 17750.7 & 79289.0 \\
\hline $143 \mathrm{~A}-1 / 2$ & 14481.1 & 13948.3 & 18806.0 & 18568.3 & 18973.7 & 84777.7 \\
\hline $1438-1$ & 15513.1 & 14980.9 & 20229.6 & 20112.0 & 20408.8 & 91244.7 \\
\hline
\end{tabular}

